\title{
On the History of Unified Field Theories
}

\author{
Hubert F. M. Goenner \\ University of Göttingen \\ Institut für Theoretische Physik \\ Tammannstr. 1 \\ D-37077 Göttingen \\ Germany \\ email: goenner@theorie.physik.uni-goettingen.de \\ http://www.theorie.physik. uni-goettingen.de/ goenner
}

Accepted on 14 January 2004

Published on 13 February 2004

\begin{abstract}
This article is intended to give a review of the history of the classical aspects of unified field theories in the 20th century. It includes brief technical descriptions of the theories suggested, short biographical notes concerning the scientists involved, and an extensive bibliography. The present first installment covers the time span between 1914 and 1933, i.e., when Einstein was living and working in Berlin - with occasional digressions into other periods. Thus, the main theme is the unification of the electromagnetic and gravitational fields augmented by shortlived attempts to include the matter field described by Schrödinger's or Dirac's equations. While my focus lies on the conceptual development of the field, by also paying attention to the interaction of various schools of mathematicians with the research done by physicists, some prosopocraphical remarks are included.
\end{abstract}




\section{Imprint / Terms of Use}

Living Reviews in Relativity is a peer reviewed open access journal published by the Max Planck Institute for Gravitational Physics, Am Mühlenberg 1, 14476 Potsdam, Germany. ISSN 1433-8351.

Because a Living Reviews article can evolve over time, we recommend to cite the article as follows:

Hubert F. M. Goenner, "On the History of Unified Field Theories",

Living Rev. Relativity, 7, (2004), 2. [Online Article]: cited [<date $>$ ], http://www.livingreviews.org/lrr-2004-2

The date given as $<$ date $>$ then uniquely identifies the version of the article you are referring to.

\section{Article Revisions}

Living Reviews supports two different ways to keep its articles up-to-date:

Fast-track revision A fast-track revision provides the author with the opportunity to add short notices of current research results, trends and developments, or important publications to the article. A fast-track revision is refereed by the responsible subject editor. If an article has undergone a fast-track revision, a summary of changes will be listed here.

Major update A major update will include substantial changes and additions and is subject to full external refereeing. It is published with a new publication number.

For detailed documentation of an article's evolution, please refer always to the history document of the article's online version at http: //www. livingreviews.org/lrr-2004-2. 


\section{Contents}

1 Introduction $\quad \mathbf{5}$

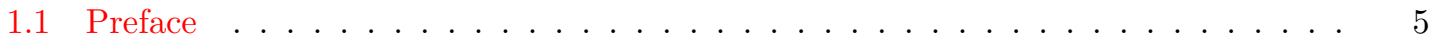

1.2 Introduction to part I . . . . . . . . . . . . . . . . 6

2 The Possibilities of Generalizing General Relativity: A Brief Overview 13

2.1 Geometry ......................... 13

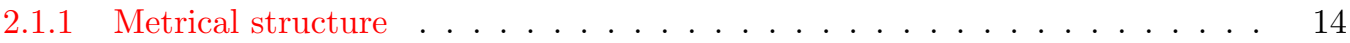

2.1 .2 Affine structure . . . . . . . . . . . . . . . . . . . . . . . . . . . . . . . .

2.1.3 Different types of geometry . . . . . . . . . . . . . . . . . . . . . . . . . . . . . . . . .

2.1 .4 Cartan's method . . . . . . . . . . . . . . . . . . . . . . . . . . . . 22

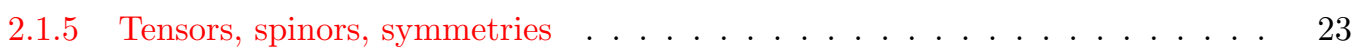

2.2 Dynamics . . . . . . . . . . . . . . . . . . . . 26

2.3 Number field . . . . . . . . . . . . . . . . . . . . . . . . . . 27

2.4 Dimension . . . . . . . . . . . . . . . . . . . . . . 28

3 Early Attempts at a Unified Field Theory $\quad 29$

3.1 First steps in the development of unified field theories . . . . . . . . . . . . . 29

3.2 Early disagreement about how to explain elementary particles by field theory . . . 31

4 The Main Ideas for Unification between about 1918 and $1923 \quad 34$

4.1 Weyl's theory .......................... 34

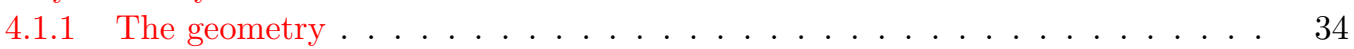

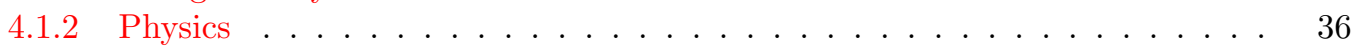

4.1.3 Reactions to Weyl's theory I: Einstein and Weyl . . . . . . . . . . . . . 38

4.1.4 Reactions to Weyl's theory II: Schouten, Pauli, Eddington, and others . . . 40

4.1.5 Reactions to Weyl's theory III: Further research . . . . . . . . . . . . . . 42

4.2 Kaluza's five-dimensional unification . . . . . . . . . . . . . . . . . . . . . . . . . . . . . . . . . . .

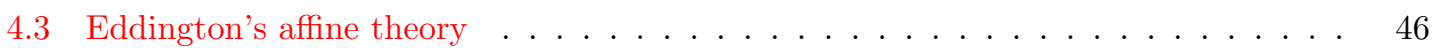

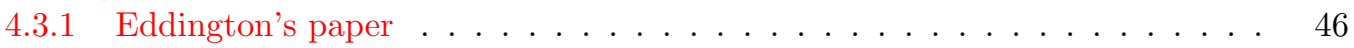

4.3.2 Einstein's reaction and publications . . . . . . . . . . . . . . 48

4.3.3 Comments by Einstein's colleagues . . . . . . . . . . . . . . . . . 52

4.3.4 Overdetermination of partial differential equations and elementary particles 53

5 Differential Geometry's High Tide $\quad 55$

6 The Pursuit of Unified Field Theory by Einstein and His Collaborators 58

6.1 Affine and mixed geometry . . . . . . . . . . . . . . . . . . 58

6.2 Further work on (metric-) affine and mixed geometry . . . . . . . . . . . . . . 61

6.3 Kaluza's idea taken up again ....................... 64

6.3 .1 Kaluza: Act I . . . . . . . . . . . . . . . . . . . . . . . 64

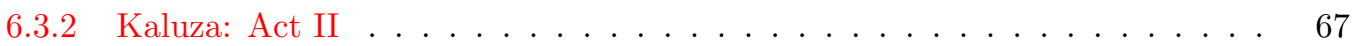

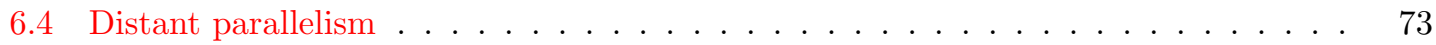

6.4.1 Cartan and Einstein . . . . . . . . . . . . . . . . . 73

6.4.2 How the word spread . . . . . . . . . . . . . . . . . . 75

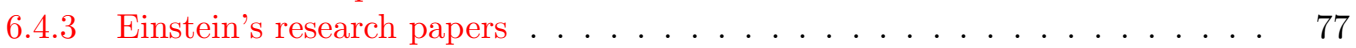

6.4.4 Reactions I: Mostly critical . . . . . . . . . . . . . . 85

6.4.5 Reactions II: Further research on distant parallelism . . . . . . . . . . . 90

6.4.6 Overdetermination and compatibility of systems of differential equations . . 96 
7 Geometrization of the Electron Field as an Additional Element of Unified Field Theory

7.1 Unification of Maxwell's and Dirac's equations, of electrons and light . . . . . . . . 100

7.2 Dirac's electron with spin, Einstein's teleparallelism, and Kaluza's fifth dimension . 102

7.2.1 Spinors . . . . . . . . . . . . . . . . . . . 102

7.2.2 General relativistic Dirac equation and unified field theory . . . . . . . . . . 104

7.2.3 Parallelism at a distance and electron spin . . . . . . . . . . . . . . . . . . 110

7.2 .4 Kaluza's theory and wave mechanics . . . . . . . . . . . . . . . . 111

7.3 Einstein, spinors, and semi-vectors . . . . . . . . . . . . . 116

8 Less Than Unification $r 20$

9 Mutual Influences Among Mathematicians and Physicists? 122

10 Public Reception of Unified Field Theory at the Time 124

11 Conclusion $\quad 125$

12 Acknowledgements 128

$\begin{array}{lr}\text { References } & 129\end{array}$ 


\section{Introduction}

\subsection{Preface}

This historical review of classical unified field theories consists of two parts. In the first, the development of unified field theory between 1914 and 1933, i.e., during the years Einstein ${ }^{1}$ lived and worked in Berlin, will be covered. In the second, the very active period after 1933 until the 1960s to 1970s will be reviewed. In the first version of Part I presented here, in view of the immense amount of material, neither all shades of unified field theory nor all the contributions from the various scientific schools will be discussed with the same intensity; I apologise for the shortcoming and promise to improve on it with the next version. At least, even if I do not discuss them all in detail, as many references as are necessary for a first acquaintance with the field are listed here; completeness may be reached only (if at all) by later updates. Although I also tried to take into account the published correspondence between the main figures, my presentation, again, is far from exhaustive in this context. Eventually, unpublished correspondence will have to be worked in, and this may change some of the conclusions. Purposely I included mathematicians and also theoretical physicists of lesser rank than those who are known to be responsible for big advances. My aim is to describe the field in its full variety as it presented itself to the reader at the time.

The review is written such that physicists should be able to follow the technical aspects of the papers (cf. Section 2), while historians of science without prior knowledge of the mathematics of general relativity at least might gain an insight into the development of concepts, methods, and scientific communities involved. I should hope that readers find more than one opportunity for further in-depth studies concerning the many questions left open.

I profited from earlier reviews of the field, or of parts of it, by Pauli ${ }^{2}$ ([246], Section V); Ludwig [212]; Whittaker ([414], pp. 188-196); Lichnerowicz [209]; Tonnelat ([356], pp. 1-14); Jordan ([176], Section III); Schmutzer ([290], Section X); Treder ([183], pp. 30-43); Bergmann ([12], pp. 6273); Straumann [334, 335]; Vizgin [384, 385] ${ }^{3}$; Bergia [11]; Goldstein and Ritter [146]; Straumann and O'Raifeartaigh [240]; Scholz [292], and Stachel [330]. The section on Einstein's unified field theories in Pais' otherwise superb book presents the matter neither with the needed historical correctness nor with enough technical precision [241]. A recent contribution of van Dongen, focussing on Einstein's methodology, was also helpful [371]. As will be seen, with regard to interpretations and conclusions, my views are different in some instances. In Einstein biographies, the subject of "unified field theories" - although keeping Einstein busy for the second half of his life - has been dealt with only in passing, e.g., in the book of Jordan [177], and in an unsatisfying way in excellent books by Fölsing [136] and by Hermann [159]. This situation is understandable; for to describe a genius stubbornly clinging to a set of ideas, sterile for physics in comparison with quantum mechan-

\footnotetext{
1 Albert Einstein (1879-1955). Born in Ulm, Württemberg (Germany). Studied physics and mathematics at the Swiss Federal Polytechnic School (ETH) Zurich and received his doctor's degree in 1905. Lecturer at the University of Bern (Switzerland), Professor in Zurich, Prague (then belonging to Austria), Berlin (Germany) and Princeton (U.S.A.). Nobel Prize 1921 for his work on the light-electric effect (photon concept). Best known for his special and general relativity theories. Important results in Brownian motion and the statistical foundations of radiation as a quantum phenomenon. Worked for more than 30 years on Unified Field Theory.

2 Wolfgang Ernst Pauli (1900-1958). Born in Vienna, Austria. Studied at the University of Munich with A. Sommerfeld who recognised his great gifts. Received his doctorate in 1921 for a thesis on the quantum theory of ionised molecular hydrogen. From October 1921 assistant of Max Born in Göttingen. After a year with Bohr, Pauli, became a lecturer at the University of Hamburg in 1923. In 1928 he was appointed professor of theoretical physics at the Federal Institute of Technology in Zürich. From 1945-1950 guest professor at the Institute for Advanced Study, Princeton. He then returned to Zürich. Did important work in quantum mechanics, quantum field theory and elementary particle theory (fourth quantum number (spin), Pauli exclusion principle, prediction of neutrino). Fellow of the Royal Society. Nobel Prize winner in 1954.

${ }^{3}$ Vizgin's book is the only one that covers the gamut of approaches during the period considered. Fortunately, he has made accessible contributions in the Russian language by scientists in the Soviet Union. Vizgin also presents and discusses attempts at unification prior to 1914 .
} 
ics, over a period of more than 30 years, is not very rewarding. For the short biographical notes, various editions of J. C. Poggendorff's Biographisch-Literarischem Handwörterbuch and internet sources have been used (in particular [1]).

If not indicated otherwise, all non-English quotations have been translated by the author; the original text of quotations is given in footnotes.

\section{$1.2 \quad$ Introduction to part I}

Past experience has shown that formerly unrelated parts of physics could be fused into one single conceptual formalism by a new theoretical perspective: electricity and magnetism, optics and electromagnetism, thermodynamics and statistical mechanics, inertial and gravitational forces. In the second half of the 20th century, the electromagnetic and weak nuclear forces have been bound together as an electroweak force; a powerful scheme was devised to also include the strong interaction (chromodynamics), and led to the standard model of elementary particle physics. Unification with the fourth fundamental interaction, gravitation, is in the focus of much present research in classical general relativity, supergravity, superstring, and supermembrane theory but has not yet met with success. These types of "unifications" have increased the explanatory power of present day physical theories and must be considered as highlights of physical research.

In the historical development of the idea of unification, i.e., the joining of previously separated areas of physical investigation within one conceptual and formal framework, two closely linked yet conceptually somewhat different approaches may be recognised. In the first, the focus is on unification of representations of physical fields. An example is given by special relativity which, as a framework, must surround all phenomena dealing with velocities close to the velocity of light in vacuum. The theory thus is said to provide "a synthesis of the laws of mechanics and of electromagnetism" ([16], p. 132). Einstein's attempts at the inclusion of the quantum area into his classical field theories belongs to this path. Nowadays, quantum field theory is such a unifying representation ${ }^{4}$. In the second approach, predominantly the unification of the dynamics of physical fields is aimed at, i.e., a unification of the fundamental interactions. Maxwell's theory might be taken as an example, unifying the electrical and the magnetic field once believed to be dynamically different. Most of the unified theories described in this review belong here: Gravitational and electromagnetic fields are to be joined into a new field. Obviously, this second line of thought cannot do without the first: A new representation of fields is always necessary.

In all the attempts at unification we encounter two distinct methodological approaches: a deductive-hypothetical and an empirical-inductive method. As Dirac pointed out, however,

"The successful development of science requires a proper balance between the method of building up from observations and the method of deducing by pure reasoning from speculative assumptions, [...]." ([233], p. 1001)

In an unsuccessful hunt for progress with the deductive-hypothetical method alone, Einstein spent decades of his life on the unification of the gravitational with the electromagnetic and, possibly, other fields. Others joined him in such an endeavour, or even preceded him, including Mie, Hilbert, Ishiwara, Nordström, and others ${ }^{5}$. At the time, another road was impossible because of the lack of empirical basis due to the weakness of the gravitational interaction. A similar situation obtains even today within the attempts for reaching a common representation of all four fundamental interactions. Nevertheless, in terms of mathematical and physical concepts, a lot has been learned even from failed attempts at unification, vid. the gauge idea, or dimensional reduction (KaluzaKlein), and much still might be learned in the future.

\footnotetext{
${ }^{4}$ The inclusion of the quantum corresponds to Vizgin's maximal unification problem [385], p. 169.

${ }^{5}$ See Section 1 of Vizgin's book [385] for a treatment of the history of pre-relativistic unified field theories and an exposition of Mie's, Ishiwara's, and Nordström's approaches.
} 
In the following I shall sketch, more or less chronologically, and by trailing Einstein's path, the history of attempts at unifying what are now called the fundamental interactions during the period from about 1914 to 1933 . Until the end of the thirties, the only accepted fundamental interactions were the electromagnetic and the gravitational, plus, tentatively, something like the "mesonic" or "nuclear" interaction. The physical fields considered in the framework of "unified field theory" including, after the advent of quantum (wave-) mechanics, the wave function satisfying either Schrödinger's or Dirac's equation, were all assumed to be classical fields. The quantum mechanical wave function was taken to represent the field of the electron, i.e., a matter field. In spite of this, the construction of quantum field theory had begun already around 1927 [52, 174, 178, 175, 179]. For the early history and the conceptual development of quantum field theory, cf. Section 1 of Schweber [322], or Section 7.2 of Cao [28]; for Dirac's contributions, cf. [190]. Nowadays, it seems mandatory to approach unification in the framework of quantum field theory.

General relativity's doing away with forces in exchange for a richer (and more complicated) geometry of space and time than the Euclidean remained the guiding principle throughout most of the attempts at unification discussed here. In view of this geometrization, Einstein considered the role of the stress-energy tensor $T^{i k}$ (the source-term of his field equations $G^{i k}=-\kappa T^{i k}$ ) a weak spot of the theory because it is a field devoid of any geometrical significance.

Therefore, the various proposals for a unified field theory, in the period considered here, included two different aspects:

- An inclusion of matter in the sense of a desired replacement, in Einstein's equations and their generalisation, of the energy-momentum tensor of matter by intrinsic geometrical structures, and, likewise, the removal of the electric current density vector as a non-geometrical source term in Maxwell's equations.

- The development of a unified field theory more geometrico for electromagnetism and gravitation, and in addition, later, of the "field of the electron" as a classical field of "de Brogliewaves" without explicitly taking into account further matter sources ${ }^{6}$.

In a very Cartesian spirit, Tonnelat (Tonnelat 1955 [356], p. 5) gives a definition of a unified field theory as

"a theory joining the gravitational and the electromagnetic field into one single hyperfield whose equations represent the conditions imposed on the geometrical structure of the universe."

No material source terms are taken into account ${ }^{7}$. If however, in this context, matter terms appear in the field equations of unified field theory, they are treated in the same way as the stress-energy tensor is in Einstein's theory of gravitation: They remain alien elements.

For the theories discussed, the representation of matter oscillated between the point-particle concept in which particles are considered as singularities of a field, to particles as everywhere regular field configurations of a solitonic character. In a theory for continuous fields as in general relativity, the concept of point-particle is somewhat amiss. Nevertheless, geodesics of the Riemannian geometry underlying Einstein's theory of gravitation are identified with the worldlines of freely moving point-particles. The field at the location of a point-particle becomes unbounded, or "singular", such that the derivation of equations of motion from the field equations is a non-trivial affair. The competing paradigm of a particle as a particular field configuration of the electromagnetic and gravitational fields later has been pursued by J. A. Wheeler under the names "geon" and

\footnotetext{
${ }^{6}$ In present-day interpretation, the first two fields are fields mediating the interactions while the third, the electron field, really is a matter field.

${ }^{7}$ This definition corresponds, in a geometrical framework, to Vizgin's minimal unification problem ([385], p. 187).
} 
"geometrodynamics" in both the classical and the quantum realm [412]. In our time, gravitational solitonic solutions also have been found [235, 26].

Even before the advent of quantum mechanics proper, in 1925-26, Einstein raised his expectations with regard to unified field theory considerably; he wanted to bridge the gap between classical field theory and quantum theory, preferably by deriving quantum theory as a consequence of unified field theory. He even seemed to have believed that the quantum mechanical properties of particles would follow as a fringe benefit from his unified field theory; in connection with his classical teleparallel theory it is reported that Einstein, in an address at the University of Nottingham, said that he

"is in no way taking notice of the results of quantum calculation because he believes that by dealing with microscopic phenomena these will come out by themselves. Otherwise he would not support the theory." ([91], p. 610)

However, in connection with one of his moves, i.e., the 5-vector version of Kaluza ${ }^{8}$ 's theory (cf. Sections $4.2,6.3$ ), which for him provided "a logical unity of the gravitational and the electromagnetic fields", he regretfully acknowledged:

"But one hope did not get fulfilled. I thought that upon succeeding to find this law, it would form a useful theory of quanta and of matter. But, this is not the case. It seems that the problem of matter and quanta makes the construction fall apart." 9 ([96], p. 442)

Thus, unfortunately, also the hopes of the eminent mathematician Schouten $^{10}$, who knew some physics, were unfulfilled:

"[...] collections of positive and negative electricity which we are finding in the positive nuclei of hydrogen and in the negative electrons. The older Maxwell theory does not explain these collections, but also by the newer endeavours it has not been possible to recognise these collections as immediate consequences of the fundamental differential equations studied. However, if such an explanation should be found, we may perhaps also hope that new light is shed on the [...] mysterious quantum orbits." ${ }^{11}$ ([301], p. 39)

In this context, through all the years, Einstein vainly tried to derive, from the field equations of his successive unified field theories, the existence of elementary particles with opposite though otherwise equal electric charge but unequal mass. In correspondence with the state of empirical knowledge at the time (i.e., before the positron was found in 1932/33), but despite theoretical

8 Theodor Franz Eduard Kaluza (1885-1954). Born in Ratibor, Germany (now Raciborz, Poland). Studied mathematics at the University of Königsberg (now Kaliningrad, Russia) and became a lecturer there in 1910. In 1929 he received a professorship at the University of Kiel, and in 1935 was made full professor at the University of Göttingen. He wrote only a handful of mathematical papers and a textbook on "Higher mathematics for the practician" (cf. [423]).

9 "Eine Hoffnung ist aber nicht in Erfüllung gegangen. Ich dachte, wenn es gelingt, dieses Gesetz aufzustellen, dass es eine brauchbare Theorie der Quanten und Materie bilden würde. Aber das ist nicht der Fall. Die Konstruktion scheint am Problem der Materie und der Quanten zu scheitern."

10 Jan Arnoldus Schouten (1883-1971). Born near Amsterdam in the Netherlands. Studied electrical engineering at the Technical University (Hogeschool) of Delft and then mathematics at the University of Leiden. His doctoral thesis of 1914 was on tensor analysis, a topic he worked on during his entire academic career. From 1914 until 1943 he held a professorship in mathematics at the University of Delft, and from 1948 to 1953 he was director of the Mathematical Research Centre at the University of Amsterdam. He was a prolific writer, applying tensor analysis to Lie groups, general relativity, unified field theory, and differential equations.

11 “[... Anhäufungen von positiver und negativer Elektrizität, die wir in den positiven Wasserstoffkernen und in den negativen Elektronen antreffen. Die ältere Maxwellsche Theorie erklärt diese Anhäufungen nicht, aber auch den neueren Bestrebungen ist es bisher nicht gelungen, diese Anhäufungen als selbstverständliche Folgen der zugrundeliegenden Differentialgleichungen zu erkennen. Sollte aber eine solche Erklärung gefunden werden, so darf man vielleicht auch hoffen, dass die [...] mysteriösen Quantenbahnen in ein neues Licht gerückt werden." 
hints pointing into a different direction to be found in Dirac's papers, he always paired electron and proton ${ }^{12}$.

Of course, by quantum field theory the dichotomy between matter and fields in the sense of a dualism is minimised as every field carries its particle-like quanta. Today's unified field theories appear in the form of gauge theories; matter is represented by operator valued spin-half quantum fields (fermions) while the "forces" mediated by "exchange particles" are embodied in gauge fields, i.e., quantum fields of integer spin (bosons). The space-time geometry used is rigidly fixed, and usually taken to be Minkowski space or, within string and membrane theory, some higher-dimensional manifold also loosely called "space-time", although its signature might not be Lorentzian and its dimension might be 10, 11, 26, or some other number larger than four. A satisfactory inclusion of gravitation into the scheme of quantum field theory still remains to be achieved.

In the period considered, mutual reservations may have existed between the followers of the new quantum mechanics and those joining Einstein in the extension of his general relativity. The latter might have been puzzled by the seeming relapse of quantum mechanics from general covariance to a mere Galilei- or Lorentz-invariance, and by the statistical interpretation of the Schrödinger wave function. Lanczos ${ }^{13}$, in 1929, was well aware of his being out of tune with those adherent to quantum mechanics:

"I therefore believe that between the 'reactionary point of view' represented here, aiming at a complete field-theoretic description based on the usual space-time structure and the probabilistic (statistical) point of view, a compromise [...] no longer is possible." ${ }^{14}$ ([198], p. 486, footnote)

On the other hand, those working in quantum theory may have frowned upon the wealth of objects within unified field theories uncorrelated to a convincing physical interpretation and thus, in principle, unrelated to observation. In fact, until the 1930s, attempts still were made to "geometrize" wave mechanics while, roughly at the same time, quantisation of the gravitational field had also been tried [284]. Einstein belonged to those who regarded the idea of unification as more fundamental than the idea of field quantisation [95]. His thinking is reflected very well in a remark made by Lanczos at the end of a paper in which he tried to combine Maxwell's and Dirac's equations:

"If the possibilities anticipated here prove to be viable, quantum mechanics would cease to be an independent discipline. It would melt into a deepened 'theory of matter' which would have to be built up from regular solutions of non-linear differential equations, in an ultimate relationship it would dissolve in the 'world equations' of the Universe. Then, the dualism 'matter-field' would have been overcome as well as the dualism 'corpuscle-wave'." 15 ([198], p. 493)

\footnotetext{
${ }^{12}$ It is true that Dirac, in his first paper, in contrast to what his "hole"-theory implied, had identified the positively charged particle corresponding to the electron also with the proton [55]. However, after Weyl had pointed out that Dirac's hole theory led to equal masses [409], he changed his mind and gave the new particle the same mass as the electron [56].

13 Cornelius Lanczos (Kornél Löwy) (1893-1974). Born in Székesfehérvár (Hungary). Studied physics and mathematics at the University of Budapest with Eötvös, Fejér, and Lax. Received his doctorate in 1921, became scientific assistant at the University of Freiburg (Germany) and lecturer at the University of Frankfurt am Main (Germany). Worked with Einstein in Berlin 1928-1929, then returned to Frankurt. Became a visiting professor at Purdue University in 1931 and came back on a professorship in 1932. Worked mainly in mathematical physics and numerical analysis. After 1944 he held various posts in industry and in the National Bureau of Standards. Left the U.S.A. during the McCarthy era and in 1952 followed an invitation by Schrödinger to become head of the Theoretical Physics Department of the Dublin Institute for Advanced Study.

14 "Ich glaube darum, dass zwischen dem hier vertretenen 'reactionären Standpunkt', der eine vollständige feldtheoretische Beschreibung auf Grund der normalen Raum-Zeit-Struktur erstrebt, und dem wahrscheinlichkeitstheoretischen (statistischen) Standpunkt ein Kompromiss [...] nicht mehr möglich ist."

15 "Sollten sich die hier vorausgeahnten Möglichkeiten als wirklich lebensfähig erweisen, so würde die Quanten-
} 
Lanczos' work shows that there has been also a smaller subprogram of unification as described before, i.e., the view that somehow the electron and the photon might have to be treated together. Therefore, a common representation of Maxwell's equations and the Dirac equation was looked for (cf. Section 7.1).

During the time span considered here, there also were those whose work did not help the idea of unification, e.g., van Dantzig ${ }^{16}$ wrote a series of papers in the first of which he stated:

"It is remarkable that not only no fundamental tensor [first fundamental form] or tensordensity, but also no connection, neither Riemannian nor projective, nor conformal, is needed for writing down the [Maxwell] equations. Matter is characterised by a bivectordensity [...]." ([367], p. 422, and also [363, 364, 365, 366])

If one of the fields to be united asks for less "geometry", why to mount all the effort needed for generalising Riemannian geometry?

A methodological weak point in the process of the establishment of field equations for unified field theory was the constructive weakness of alternate physical limits to be taken:

- no electromagnetic field $\rightarrow$ Einstein's equations in empty space;

- no gravitational field $\rightarrow$ Maxwell's equations;

- "weak" gravitational and electromagnetic fields $\rightarrow$ Einstein-Maxwell equations;

- no gravitational field but a "strong" electromagnetic field $\rightarrow$ some sort of non-linear electrodynamics.

A similar weakness occurred for the equations of motion; about the only limiting equation to be reproduced was Newton's equation augmented by the Lorentz force. Later, attempts were made to replace the relationship "geodesics $\rightarrow$ freely falling point particles" by more general assumptions for charged or electrically neutral point particles - depending on the more general (non-Riemannian) connections introduced ${ }^{17}$. A main hindrance for an eventual empirical check of unified field theory was the persistent lack of a worked out example leading to a new gravito-electromagnetic effect.

In the following Section 2, a multitude of geometrical concepts (affine, conformal, projective spaces, etc.) available for unified field theories, on the one side, and their use as tools for a description of the dynamics of the electromagnetic and gravitational field on the other will be sketched. Then, we look at the very first steps towards a unified field theory taken by Reichenbächer ${ }^{18}$,

mechanik aufhören, eine selbständige Disziplin zu sein. Sie würde verschmelzen mit einer vertieften 'Theorie der Materie', die auf reguläre Lösungen von nicht-linearen Differentialgleichungen aufzubauen hätte, - in letztem Zusammenhang also aufgehen in den 'Weltgleichungen' des Universums. Der Dualismus 'Materie-Feld' würde dann ebenso überwunden sein, wie der Dualismus 'Korpuskel-Welle'."

16 David van Dantzig (1900-1959). Born in Rotterdam, Netherlands. Studied mathematics at the University of Amsterdam. Worked first on differential geometry, electrodynamics and unified field theory. Known as co-founder, in 1946, of the Mathematical Centre in Amsterdam and by his role in establishing mathematical statistics as a subdiscipline in the Netherlands.

${ }^{17}$ Thus, in a paper of 1934 really belonging to the 2 nd part of this review, Schouten and Haantjes exchanged the previously assigned "induced geodesic lines" for "auto-geodesical lines" [311].

18 Ernst Reichenbächer (1881-1944). Studied mathematics and received his doctorate from the University of Halle in 1903 under the guidance of Albert Wangerin (a student of Franz Neumann in Königsberg). At first, Reichenbächer did not enter an academic career, but started teaching in a Gymnasium in Wilhelmshaven in North Germany, then in Königsberg on the Baltic Sea. In 1929 he became a Privatdozent (lecturer) at the University of Königsberg (now Kaliningrad, Russia). His courses covered special and general relativity, the physics of fixed stars and galaxies with a touch on cosmology, and quantum mechanics. In the fifth year of World War II he finally received the title of professor at the University Königsberg, but in the same year was killed during a bombing raid on the city. 
Förster (alias Bach), Weyl ${ }^{19}$, Eddington ${ }^{20}$, and Einstein (see Section 3.1). In Section 4, the main ideas are developed. They include Weyl's generalization of Riemannian geometry by the addition of a linear form (see Section 4.1) and the reaction to this approach. To this, Kaluza's idea concerning a geometrization of the electromagnetic and gravitational fields within a five-dimensional space will be added (see Section 4.2) as well as the subsequent extensions of Riemannian to affine geometry by Schouten, Eddington, Einstein, and others (see Section 4.3). After a short excursion to the world of mathematicians working on differential geometry (see Section 5), the research of Einstein and his assistants is studied (see Section 6). Kaluza's theory received a great deal of attention after O. Klein ${ }^{21}$ intervention and extension of Kaluza's paper (see Section 6.3.2). Einstein's treatment of a special case of a metric-affine geometry, i.e., "distant parallelism", set off an avalanche of research papers (see Section 6.4.4), the more so as, at the same time, the covariant formulation of Dirac's equation was a hot topic. The appearance of spinors in a geometrical setting, and endeavours to link quantum physics and geometry (in particular, the attempt to geometrize wave mechanics) are also discussed (see Section 7). We have included this topic although, strictly speaking, it only touches the fringes of unified field theory.

In Section 9, particular attention is given to the mutual influence exerted on each other by the Princeton (Eisenhart ${ }^{22}$, Veblen $^{23}$ ), French $\left(\right.$ Cartan $^{24}$ ), and the Dutch (Schouten, Struik ${ }^{25}$ ) schools

${ }^{19}$ Hermann Klaus Hugo Weyl (1885-1955). Born in Elmshorn, Germany. Studied at the Universities of Munich and Göttingen where he received his doctorate in 1908 (Hilbert was his supervisor). From 1913 he held the Chair of Mathematics at the Federal Institute of Technology in Zürich, and from 1930 to 1933 a corresponding Chair at the University of Göttingen. Then until retirement he worked at the Institute for Advanced Study in Princeton. Weyl made important contributions in mathematics (integral equations, Riemannian surfaces, continuous groups, analytic number theory) and theoretical physics (differential geometry, unified field theory, gauge theory). For his papers, cf. also the Collected Works [411]

20 Arthur Stanley Eddington (1882-1944). Born in Kendal, England. Studied mathematics at Owens College, Manchester and Trinity College, Cambridge. After some work in physics, moved into astronomy in 1905 and was appointed to the Royal Observatory at Greenwich. From 1914 director of the Cambridge Observatory. Fellow of the Royal Society. As a Quaker he became a conscientious objector to military service during the First World War. Eddington made important contributions to general relativity and astrophysics (internal structure of stars). In 1918, he led an eclipse expedition from which the first indications resulted that Einstein's general relativity theory was correct. Wrote also on epistemology and the philosophy of science.

${ }^{21}$ Oskar Klein (1894-1977). Born in Mörby, Sweden. After work with Arrhenius in physical chemistry, he met Kramers, then a student of Bohr, in 1917. Klein worked with Bohr in the field of molecular physics and received his doctorate in 1921 at Stockholm Högskola. His first research position was at the University of Michigan in Ann Arbor, where he worked on the Zeeman effect. Back in Europe from 1925, he taught at Lund University and tried to connect Kaluza's work with quantum theory. In 1930 he became professor for mathematical physics at Stockholm Högskola until retirement. His later work included quantum theory (Klein-Nishina formula), superconductivity, and cosmology.

${ }^{22}$ Luther Pfahler Eisenhart (1876-1965). Born in York, Pennsylvania, U.S.A. Studied mathematics at John Hopkins University, Baltimore and received his doctorate in 1900. Eisenhart taught at the University of Princeton from 1900, was promoted to professor in 1909 and remained there (as Dean of the mathematical Faculty and Dean of the Graduate School) until his retirement in 1945. All his work is in differential geometry, including Riemannian and non-Riemannian geometry and in group theory.

23 Oswald Veblen (1880-1960). Born in Decorah, Iowa, U.S.A. Entered the University of Iowa in 1894, receiving his B.A. in 1898. He obtained his doctorate from the University of Chicago on "a system of axioms in geometry" in 1903. He taught mathematics at Princeton (1905-1932), at Oxford in 1928-1929, and became a professor at the Institute for Advanced Study in Princeton in 1932. Veblen made important contribution to projective and differential geometry, and to topology. He gave a new treatment of spin.

${ }^{24}$ Elie Joseph Cartan (1869-1951). Born in Dolomien near Chambéry, France. Student at l'École Normale since 1888, he received his Ph.D. in 1894 with a thesis in which he completed Killing's classification of semisimple algebras. He lectured at Montpellier (1894-1903), Lyon (1896-1903), Nancy (1903-1909), and Paris (1909-1940). His following work on the representation of semisimple Lie groups combines group theory, classical geometry, differential geometry, and topology. From 1904 he worked on differential equations and differential geometry, and developed a theory of moving frames (calculus of differential forms). He also contributed to the geometry of symmetric spaces and published on general relativity and its geometric extensions as well as on the theory of spinors. For his Collected Works, cf. [38].

25 Dirk J. Struik (1894-2000). Born in Rotterdam in the Netherlands. Studied mathematics and physics at the University of Leiden with Lorentz and de Sitter. Received his doctorate in 1922. Then worked with Schouten at 
of mathematicians, and the work of physicists such as Eddington, Einstein, their collaborators, and others. In Section 10, the reception of unified field theory at the time is briefly discussed.

the University of Delft and, with a Rockefeller International Education Fellowship, moved to Rome and Göttingen. After a collaboration with Wiener, in 1926 he received a lectureship at the Massachusetts Institute of Technology (MIT) in Cambridge, Ma. where he became full professor in 1940. He stayed on the MIT mathematics faculty until 1960. As a professed Marxist he was suspended from teaching duties during the McCarthy period but was reinstated in 1956. In 1972, he became an honorary research associate in the History of Science Department of Harvard University. 


\section{The Possibilities of Generalizing General Relativity: A Brief Overview}

As a rule, the point of departure for unified field theory was general relativity. The additional task then was to "geometrize" the electromagnetic field. In this review, we will encounter essentially five different ways to include the electromagnetic field into a geometric setting:

- by connecting an additional linear form to the metric through the concept of "gauging" (Weyl);

- by introducing an additional space dimension (Kaluza);

- by choosing an asymmetric Ricci tensor (Eddington);

- by adding an antisymmetric tensor to the metric (Bach, Einstein);

- by replacing the metric by a 4-bein field (Einstein).

In order to bring some order into the wealth of these attempts towards "unified field theory," I shall distinguish four main avenues extending general relativity, according to their mathematical direction: generalisation of

- geometry,

- dynamics (Lagrangians, field equations),

- number field, and

- dimension of space,

as well as their possible combinations. In the period considered, all four directions were followed as well as combinations between them like e.g., five-dimensional theories with quadratic curvature terms in the Lagrangian. Nevertheless, we will almost exclusively be dealing with the extension of geometry and of the number of space dimensions.

\subsection{Geometry}

It is very easy to get lost in the many constructive possibilities underlying the geometry of unified field theories. We briefly describe the mathematical objects occurring in an order that goes from the less structured to the more structured cases. In the following, only local differential geometry is taken into account ${ }^{26}$.

The space of physical events will be described by a real, smooth manifold $M_{D}$ of dimension $D$ coordinatised by local coordinates $x^{i}$, and provided with smooth vector fields $X, Y, \ldots$ with components $X^{i}, Y^{i}, \ldots$ and linear forms $\omega, \nu, \ldots,\left(\omega_{i}, \nu_{i}\right)$ in the local coordinate system, as well as further geometrical objects such as tensors, spinors, connections ${ }^{27}$. At each point, $D$ linearly independent vectors (linear forms) form a linear space, the tangent space (cotangent space) of $M_{D}$. We will assume that the manifold $M_{D}$ is space- and time-orientable. On it, two independent fundamental structural objects will now be introduced.

\footnotetext{
${ }^{26}$ For the following, it is assumed that readers have some prior knowledge of the mathematics underlying General Relativity.

${ }^{27}$ For the precise definition of "geometrical object", cf. Yano's book [425].
} 


\subsubsection{Metrical structure}

The first is a prescription for the definition of the distance $d s$ between two infinitesimally close points on $M_{D}$, eventually corresponding to temporal and spatial distances in the external world. For $d s$, we need positivity, symmetry in the two points, and the validity of the triangle equation. We know that $d s$ must be homogeneous of degree one in the coordinate differentials $d x^{i}$ connecting the points. This condition is not very restrictive; it still includes Finsler geometry [281, 126, 224] to be briefly touched, below.

In the following, $d s$ is linked to a non-degenerate bilinear form $g(X, Y)$, called the first fundamental form; the corresponding quadratic form defines a tensor field, the metrical tensor, with $D^{2}$ components $g_{i j}$ such that

$$
d s=\sqrt{g_{i j} d x^{i} d x^{j}},
$$

where the neighbouring points are labeled by $x^{i}$ and $x^{i}+d x^{i}$, respectively ${ }^{28}$. Besides the norm of a vector $|X|:=\sqrt{g_{i j} X^{i} X^{j}}$, the "angle" between directions $X, Y$ can be defined by help of the metric:

$$
\cos (\angle(X, Y)):=\frac{g_{i j} X^{i} Y^{j}}{|X||Y|} .
$$

From this we note that an antisymmetric part of the metrical tensor does not influence distances and norms but angles.

With the metric tensor having full rank, its inverse $g^{i k}$ is defined through ${ }^{29}$

$$
g_{m i} g^{m j}=\delta_{i}^{j}
$$

We are used to $g$ being a symmetric tensor field, i.e., with $g_{i k}=g_{(i k)}$ and with only $D(D+1) / 2$ components; in this case the metric is called Riemannian if its eigenvalues are positive (negative) definite and Lorentzian if its signature is $\pm(D-2)^{30}$. In the following this need not hold, so that the decomposition obtains ${ }^{31}$ :

$$
g_{i k}=\gamma_{(i k)}+\phi_{[i k]} .
$$

An asymmetric metric was considered in one of the first attempts at unifying gravitation and electromagnetism after the advent of general relativity.

For an asymmetric metric, the inverse

$$
g^{i k}=h^{(i k)}+f^{[i k]}=h^{i k}+f^{i k}
$$

is determined by the relations

$$
\gamma_{i j} \gamma^{i k}=\delta_{j}^{k}, \quad \phi_{i j} \phi^{i k}=\delta_{j}^{k}, \quad h_{i j} h^{i k}=\delta_{j}^{k}, \quad f_{i j} f^{i k}=\delta_{j}^{k},
$$

and turns out to be [356]

$$
\begin{aligned}
h^{(i k)} & =\frac{\gamma}{g} \gamma^{i k}+\frac{\phi}{g} \phi^{i m} \phi^{k n} \gamma_{m n}, \\
f^{(i k)} & =\frac{\phi}{g} \phi^{i k}+\frac{\gamma}{g} \gamma^{i m} \gamma^{k n} \phi_{m n},
\end{aligned}
$$

\footnotetext{
${ }^{28}$ The second fundamental form comes into play when local isometric embedding is considered, i.e., when $M_{D}$ is taken as a submanifold of a larger space such that the metrical relationships are conserved. In the following, all geometrical objects are supposed to be differentiable as often as is needed.

${ }^{29}$ Here, the Kronecker-symbol $\delta^{i}{ }_{k}$ with value +1 for $i=k$, and value 0 for $i \neq k$ is used. $\delta^{i}{ }_{k}$ keeps its components unchanged under arbitrary coordinate transformations.

${ }^{30}$ Latin indices $i, j, k, \ldots$ run from 1 to $D$, or from 0 to $D-1$ to point to the single timelike direction. We have used the symmetrisation bracket defined by $A_{(i j)}:=1 / 2\left(A_{i j}+A_{j i}\right)$.

${ }^{31}$ In physical applications, special conditions for $\gamma$ and $\phi$ might be needed in order to guarantee that $g$ is a Lorentz metric.
} 
where $g, \phi$, and $\gamma$ are the determinants of the corresponding tensors $g_{i k}, \phi_{i k}$, and $\gamma_{i k}$. We also note that

$$
g=\gamma+\phi+\frac{\gamma}{2} \gamma^{k l} \gamma^{m n} \phi_{k m} \phi_{l n}
$$

where $g:=\operatorname{det} g_{i k}, \phi:=\operatorname{det} \phi_{i k}, \gamma:=\operatorname{det} \gamma_{i k}$. The results $(6,7,8)$ were obtained already by Reichenbächer ([273], pp. 223-224) ${ }^{32}$ and also by Schrödinger [320]. Eddington also calculated Equation (8); in his expression the term $\sim \phi_{i k}{ }^{\star} \phi^{i k}$ is missing (cf. [59], p. 233).

The manifold is called space-time if $D=4$ and the metric is symmetric and Lorentzian, i.e., symmetric and with signature $\operatorname{sig} g= \pm 2$. Nevertheless, sloppy contemporaneaous usage of the term "space-time" includes arbitrary dimension, and sometimes is applied even to metrics with arbitrary signature.

In a manifold with Lorentzian metric, a non-trivial real conformal structure always exists; from the equation

$$
g(X, X)=0
$$

results an equivalence class of metrics $\{\lambda g\}$ with $\lambda$ being an arbitrary smooth function. In view of the physical interpretation of the light cone as the locus of light signals, a causal structure is provided by the equivalence class of metrics [67]. For an asymmetric metric, this structure can exist as well; it then is determined by the symmetric part $\gamma_{i k}=\gamma_{(i k)}$ of the metric alone taken to be Lorentzian.

A special case of a space with a Lorentzian metric is Minkowski space, whose metrical components, in Cartesian coordinates, are given by

$$
\eta_{i k}=\delta_{i}{ }^{0} \delta_{i}{ }^{0}-\delta_{i}{ }^{1} \delta_{i}{ }^{1}-\delta_{i}{ }^{2} \delta_{i}{ }^{2}-\delta_{i}{ }^{3} \delta_{i}{ }^{3}
$$

A geometrical characterization of Minkowski space as an uncurved, flat space is given below. Let $\mathcal{L}_{X}$ be the Lie derivative with respect to the tangent vector $\mathrm{X}^{33}$; then $\mathcal{L}_{X_{p}} \eta_{i k}=0$ holds for the Lorentz group of generators $X_{p}$.

The metric tensor $g$ may also be defined indirectly through $D$ vector fields forming an orthonormal $D$-leg (-bein) $h_{\hat{\imath}}^{k}$ with

$$
g_{l m}=h_{l \hat{\jmath}} h_{m \hat{k}} \eta^{\hat{\jmath} \hat{k}}
$$

where the hatted indices ("bein-indices") count the number of legs spanning the tangent space at each point $(\hat{\jmath}=1,2, \ldots, D)$ and are moved with the Minkowski metric ${ }^{34}$. From the geometrical point of view, this can always be done (cf. theories with distant parallelism). By introducing 1-forms $\theta^{\hat{k}}:=h_{l}^{\hat{k}} d x^{l}$, Equation (11) may be brought into the form $d s^{2}=\theta^{\hat{\imath}} \theta^{\hat{k}} \eta_{\hat{\imath} \hat{k}}$.

A new physical aspect will come in if the $h_{\hat{\imath}}^{k}$ are considered to be the basic geometric variables satisfying field equations, not the metric. Such tetrad-theories (for the case $D=4$ ) are described well by the concept of fibre bundle. The fibre at each point of the manifold contains, in the case of an orthonormal $D$-bein (tetrad), all $D$-beins (tetrads) related to each other by transformations of the group $O(D)$, or the Lorentz group, and so on.

In Finsler geometry, the line element depends not only on the coordinates $x^{i}$ of a point on the manifold, but also on the infinitesimal elements of direction between neighbouring points $d x^{i}$ :

$$
d s^{2}=g_{i j}\left(x^{n}, d x^{m}\right) d x^{i} d x^{j} .
$$

Again, $g_{i j}$ is required to be homogeneous of rank 1 .

\footnotetext{
${ }^{32}$ Reichenbächer's results agree with Schrödinger's, but, as they are in a different form than that given by Tonnelat [356], I have not checked whether they agree with Equations $(6,7)$. Neither Schrödinger nor Tonnelat give credit to Reichenbächer.

${ }^{33}$ We have $\left(L_{X} Y\right)^{k}:=([X, Y])^{k}=X^{i} \partial_{i} Y^{k}-Y^{i} \partial_{i} X^{k}$.

${ }^{34}$ Eisenhart called his object "ennuple" instead of $n$-bein [119]. In French, the expressions " $n$-pode", " $n$-èdre", and "polyaxe à $n$ dimensions" were also used.
} 


\subsubsection{Affine structure}

The second structure to be introduced is a linear connection $L$ with $D^{3}$ components $L_{i j}{ }^{k}$; it is a geometrical object but not a tensor field and its components change inhomogeneously under local coordinate transformations ${ }^{35}$. The connection is a device introduced for establishing a comparison of vectors in different points of the manifold. By its help, a tensorial derivative $\nabla$, called covariant derivative is constructed. For each vector field and each tangent vector it provides another unique vector field. On the components of vector fields $X$ and linear forms $\omega$ it is defined by

$$
\stackrel{+}{\nabla}_{k} X^{i}=\frac{\partial X^{i}}{\partial x^{k}}+L_{k j}^{i} X^{j}, \quad \stackrel{+}{\nabla}_{k} \omega_{i}=\frac{\partial \omega_{i}}{\partial x^{k}}-L_{k i}{ }^{j} \omega_{j} .
$$

The expressions $\stackrel{+}{\nabla}_{k} X^{i}$ and $\frac{\partial X^{i}}{\partial x^{k}}$ are abbreviated by $X^{i}{ }_{\| k}$ and $X^{i}{ }_{, k}$, respectively, while for a scalar $f$ covariant and partial derivative coincide: $\nabla_{i} f=\frac{\partial f}{\partial x_{i}} \equiv \partial_{i} f \equiv f_{, i}$.

We have adopted the notational convention used by Schouten [300, 310, 389]. Eisenhart and others [121, 234] change the order of indices of the components of the connection:

$$
\bar{\nabla}_{k} X^{i}=\frac{\partial X^{i}}{\partial x^{k}}+L_{j k}{ }^{i} X^{j}, \quad \bar{\nabla}_{k} \omega_{i}=\frac{\partial \omega_{i}}{\partial x^{k}}-L_{i k}{ }^{j} \omega_{j} .
$$

As long as the connection is symmetric, this does not make any difference as $\stackrel{+}{\nabla}_{k} X^{i}-\bar{\nabla}_{k} X^{i}=2 L_{[k j]}^{i} X^{j}$. For both kinds of derivatives we have:

$$
\stackrel{+}{\nabla}_{k}\left(v^{l} w_{l}\right)=\frac{\partial\left(v^{l} w_{l}\right)}{\partial x^{k}}, \quad \bar{\nabla}_{k}\left(v^{l} w_{l}\right)=\frac{\partial\left(v^{l} w_{l}\right)}{\partial x^{k}}
$$

Both derivatives are used in versions of unified field theory by Einstein and others ${ }^{36}$.

A manifold provided with only a linear connection $\mathrm{L}$ is called affine space. From the point of view of group theory, the affine group (linear inhomogeneous coordinate transformations) plays a special role: With regard to it the connection transforms as a tensor (cf. Section 2.1.5).

For a vector density (cf. Section 2.1.5), the covariant derivative of $\hat{X}$ contains one more term:

$$
\stackrel{+}{\nabla}_{k} \hat{X}^{i}=\frac{\partial \hat{X}^{i}}{\partial x^{k}}+L_{k j}{ }^{i} \hat{X}^{j}-L_{k r}{ }^{r} \hat{X}^{i}, \quad \bar{\nabla}_{k} \hat{X}^{i}=\frac{\partial X^{i}}{\partial x^{k}}+L_{j k}{ }^{i} \hat{X}^{j}-L_{r k}{ }^{r} \hat{X}^{i} .
$$

A smooth vector field $Y$ is said to be parallely transported along a parametrised curve $\lambda(u)$ with tangent vector $X$ if for its components $Y^{i}{ }_{\| k} X^{k}(u)=0$ holds along the curve. A curve is called an autoparallel if its tangent vector is parallely transported along it at each point ${ }^{37}$ :

$$
X^{i}{ }_{\| k} X^{k}(u)=\sigma(u) X^{i}
$$

By a particular choice of the curve's parameter, $\sigma=0$ may be imposed.

A transformation mapping autoparallels to autoparallels is given by:

$$
L_{i k}^{j} \rightarrow L_{i k}{ }^{j}+\delta^{j}{ }_{(i} \omega_{k)} \text {. }
$$

The equivalence class of autoparallels defined by Equation (18) defines a projective structure on $M_{D}[404,403]$.

\footnotetext{
${ }^{35}$ In an arbitrary basis for the differential forms (cotangent space), the connection may be represented by a 1-form.

${ }^{36}$ In the literature, different notations and conventions are used. Tonnelat [356] writes $A_{k ; j}:=A_{k, j}-L_{k j}{ }^{l} A_{l}$, and $A_{k ; j}:=A_{k, j}-L_{j k}^{l} A_{l}$.

${ }^{37}$ Many authors replace "autoparallel" by "geodesic". We will reserve the name geodesic for curves of extreme length; cf. Riemannian geometry.
} 
The particular set of connections

$$
{ }_{(p)} L_{i j}{ }^{k}:=L_{i j}{ }^{k}-\frac{2}{D+1} \delta^{k}{ }_{(i} L_{j)}
$$

with $L_{j}:=L_{i m}^{m}$ is mapped into itself by the transformation (18) [348].

In Part II of this article, we shall find the set of transformations $L_{i k}{ }^{j} \rightarrow L_{i k}{ }^{j}+\delta^{j} i \frac{\partial \omega}{\partial x^{k}}$ playing a role in versions of Einstein's unified field theory.

From the connection $L_{i j}{ }^{k}$ further connections may be constructed by adding an arbitrary tensor field $T$ to its symmetrised part ${ }^{38}$ :

$$
\bar{L}_{i j}{ }^{k}=L_{(i j)}{ }^{k}+T_{i j}{ }^{k}=\Gamma_{i j}{ }^{k}+T_{i j}{ }^{k} .
$$

By special choice of $T$ we can regain all connections used in work on unified field theories. We will encounter examples in later sections. The antisymmetric part of the connection, i.e.,

$$
S_{i j}^{k}=L_{[i j]}^{k}=T_{[i j]}^{k}
$$

is called torsion; it is a tensor field. The trace of the torsion tensor $S_{i}:=S_{i l}{ }^{l}$ is called torsion vector; it connects to the two traces of the affine connection $L_{i}:=L_{i l}{ }^{l} ; \tilde{L}_{j}:=L_{l j}{ }^{l}$, as $S_{i}=\frac{1}{2}\left(L_{i}-\tilde{L}_{i}\right)$.

\subsubsection{Different types of geometry}

2.1.3.1 Affine geometry Various subcases of affine spaces will occur, dependent on whether the connection is asymmetric or symmetric, i.e., with $L_{i j}{ }^{k}=\Gamma_{i j}{ }^{k}$. In physical applications, a metric always seems to be needed; hence in affine geometry it must be derived solely by help of the connection or, rather, by tensorial objects constructed from it. This is in stark contrast to Riemannian geometry where, vice versa, the connection is derived from the metric. Such tensorial objects are the two affine curvature tensors defined by ${ }^{39}$

$$
\begin{aligned}
& \stackrel{+}{K}^{i}{ }_{j k l}=\partial_{k} L_{l j}{ }^{i}-\partial_{l} L_{k j}{ }^{i}+L_{k m}{ }^{i} L_{l j}{ }^{m}-L_{l m}{ }^{i} L_{k j}{ }^{m}, \\
& \bar{K}_{j k l}^{i}=\partial_{k} L_{j l}{ }^{i}-\partial_{l} L_{j k}{ }^{i}+L_{m k}{ }^{i} L_{j l}{ }^{m}-L_{m l}{ }^{i} L_{j k}{ }^{m} \text {, }
\end{aligned}
$$

respectively. In a geometry with symmetric affine connection both tensors coincide because of

$$
\frac{1}{2}\left(\stackrel{+}{K}_{j k l}^{i}-\bar{K}_{j k l}^{i}\right)=\partial_{[k} S_{] l j}^{i}+2 S_{j[k}^{m} S_{l] m}^{i}+L_{m[k}^{i} S_{l] j}^{m}-L_{j[k}^{m} S_{l] m}^{i} .
$$

In particular, in Riemannian geometry, both affine curvature tensors reduce to the one and only Riemann curvature tensor.

The curvature tensors arise because the covariant derivative is not commutative and obeys the Ricci identity:

$$
\begin{aligned}
\stackrel{+}{\nabla}_{[j} \stackrel{+}{\nabla}_{k]} A^{i} & =\frac{1}{2} \stackrel{+}{K}_{r j k}^{i} A^{r}-S_{j k}^{r} \stackrel{+}{\nabla}_{r} A^{i} \\
\bar{\nabla}_{[j} \bar{\nabla}_{k]} A^{i} & =\frac{1}{2} \bar{K}_{r j k}^{i} A^{r}+S_{j k}^{r} \bar{\nabla}_{r} A^{i}
\end{aligned}
$$

For a vector density, the identity is given by

$$
\stackrel{+}{\nabla}_{[j} \stackrel{+}{\nabla}_{k]} \hat{A}^{i}=\frac{1}{2} \stackrel{+}{K}_{r j k}^{i} \hat{A}^{r}-S_{j k}^{r} \stackrel{+}{\nabla}_{r} \hat{A}^{i}+\frac{1}{2} V_{j k} \hat{A}^{i},
$$

\footnotetext{
${ }^{38}$ In the following we will note a symmetrical connection by $\Gamma_{i j}{ }^{k}=L_{(i j)}{ }^{k}$.

${ }^{39}$ Schouten denotes the curvature tensor by $K_{j k l}{ }^{i}$.
} 
with the homothetic curvature $V_{j k}$ to be defined below in Equation (31).

The curvature tensor (22) satisfies two algebraic identities:

$$
\begin{aligned}
\stackrel{+}{K}^{i}{ }_{j[k l]} & =0, \\
\stackrel{+}{K}^{i} & { }_{\{j k l\}}=2 \nabla_{\{j} S_{k l\}}{ }^{i}+4 S_{m\{j}{ }^{i} S_{k l\}}{ }^{m},
\end{aligned}
$$

where the curly bracket denotes cyclic permutation:

$$
K_{\{j k l\}}^{i}:=K_{j k l}^{i}+K_{l j k}^{i}+K_{k l j}^{i} .
$$

These identities can be found in Schouten's book of 1924 ([300], p. 88, 91) as well as the additional single integrability condition, called Bianchi identity:

$$
\stackrel{+}{K}^{i}{ }_{j\{k l \| m\}}=2 K_{r\{k l}^{i} S_{m\} j}{ }^{r} .
$$

A corresponding condition obtains for the curvature tensor $\bar{K}$ from Equation (23).

From both affine curvature tensors we may form two different tensorial traces each. In the first case $V_{k l}:=K_{i k l}^{i}=V_{[k l]}$, and $K_{j k}:=K_{j k i}^{i}$. $V_{k l}$ is called homothetic curvature, while $K_{j k}$ is the first of the two affine generalisations from $\stackrel{+}{K}$ and $\bar{K}$ of the Ricci tensor in Riemannian geometry. We $\operatorname{get}^{40}$

$$
V_{k l}=\partial_{k} L_{l}-\partial_{l} L_{k}
$$

and the following identities hold:

$$
\begin{aligned}
V_{k l}+2 K_{[k l]} & =4 \nabla_{[k} S_{l]}+8 S_{k l}^{m} S_{m}+2 \nabla_{m} S_{k l}^{m}, \\
\bar{V}_{k l}+2 \bar{K}_{[k l]} & =-4 \bar{\nabla}_{[k} S_{l]}+8 S_{k l}^{m} S_{m}+2 \nabla_{m} S_{k l}^{m},
\end{aligned}
$$

where $S_{k}:=S_{k l}^{l}$. While $V_{k l}$ is antisymmetric, $K_{j k}$ has both tensorial symmetric and antisymmetric parts:

$$
\begin{aligned}
& K_{[k l]}=-\partial_{[k} \tilde{L}_{l]}+\nabla_{m} S_{k l}^{m}+L_{m} S_{k l}^{m}+2 L_{[l \mid r}^{m} S_{m \mid k]}^{r}, \\
& K_{(k l)}=\partial_{(k} \tilde{L}_{l)}-\partial_{m} L_{(k l)}^{m}-\tilde{L}_{m} L_{(k l)}^{m}+L_{(k|m|}^{m} L_{l) n}^{m} .
\end{aligned}
$$

We use the notation $A_{(i|k| l)}$ in order to exclude the index $k$ from the symmetrisation bracket ${ }^{41}$.

In order to shorten the presentation of affine geometry, we refrain from listing the corresponding set of equations for the other affine curvature tensor (cf., however, [356]).

For a symmetric affine connection, the preceding results reduce considerably due to $S_{k l}{ }^{m}=0$. From Equations $(29,30,32)$ we obtain the identities:

$$
\begin{aligned}
K_{\{j k l\}}^{i} & =0, \\
K^{i}{ }_{j\{k l \| m\}} & =0, \\
V_{k l}+2 K_{[k l]} & =0,
\end{aligned}
$$

${ }^{40}$ Again, we have two kinds of homothetic curvatures deriving from $\stackrel{+}{K}$ and $\bar{K}$. In the following, we will mostly use the $\stackrel{+}{X}$-quantities and drop the + sign.

${ }^{41}$ For a three-index-tensor the symmetrisation bracket is defined by

$$
A_{(i k l)}:=\frac{1}{3 !}\left(A_{i k l}+A_{l i k}+A_{k l i}+A_{k i l}+A_{l k i}+A_{i l k}\right) .
$$


i.e., only one independent trace tensor of the affine curvature tensor exists. For the antisymmetric part of the Ricci tensor $K_{[k l]}=-\partial_{[k} \tilde{L}_{l]}$ holds. This equation will be important for the physical interpretation of affine geometry.

In affine geometry, the simplest way to define a fundamental tensor is to set $g_{i j}:=\alpha K_{(i j)}$, or $g_{i j}:=\alpha \bar{K}_{(i j)}$. It may be desirable to derive the metric from a Lagrangian; then the simplest scalar density that could be used as such is given by $\operatorname{det}\left(K_{i j}\right)^{42}$.

As a final result in this section, we give the curvature tensor calculated from the connection $\bar{L}_{i j}{ }^{k}=\Gamma_{i j}{ }^{k}+T_{i j}{ }^{k}$ (cf. Equation (20)), expressed by the curvature tensor of $\Gamma_{i j}{ }^{k}$ and by the tensor $T_{i j}{ }^{k}$ :

$$
K_{j k l}^{i}(\bar{L})=K_{j k l}^{i}(\Gamma)+2^{(\Gamma)} \nabla_{[k} T_{l] j}^{i}-2 T_{[k|j|}{ }^{m} T_{l] m}{ }^{i}+2 S_{k l}{ }^{m} T_{m j}{ }^{i},
$$

where ${ }^{(\Gamma)} \nabla$ is the covariant derivative formed with the connection $\Gamma_{i j}{ }^{k}$ (cf. also [310], p. 141).

2.1.3.2 Mixed geometry A manifold carrying both structural elements, i.e., metric and connection, is called a metric-affine space. If the first fundamental form is taken to be asymmetric, i.e., to contain an antisymmetric part $g_{[i k]}:=\frac{1}{2}\left(g_{i j}-g_{j i}\right)$, we speak of a mixed geometry. In principle, both metric-affine space and mixed geometry may always be re-interpreted as Riemannian geometry with additional geometric objects: the 2-form field $\phi(f)$ (symplectic form), the torsion $S$, and the non-metricity $Q$ (cf. Equation 41). It depends on the physical interpretation, i.e., the assumed relation between mathematical objects and physical observables, which geometry is the most suitable.

From the symmetric part of the first fundamental form $h_{i j}=g_{(i j)}$, a connection may be constructed, often called after Levi-Civita ${ }^{43}$ [204],

$$
\left\{\begin{array}{l}
k \\
i j
\end{array}\right\}:=\frac{1}{2} \gamma^{k l}\left(\gamma_{l i, j}+\gamma_{l j, i}-h_{i j,}\right),
$$

and from it the Riemannian curvature tensor defined as in Equation (22) with $L_{i j}{ }^{k}=\left\{\begin{array}{l}k \\ i j\end{array}\right\}$ (cf. Section 2.1.3); $\left\{\begin{array}{l}k \\ i j\end{array}\right\}$ is called the Christoffel symbol. Thus, in metric-affine and in mixed geometry, two different connections arise in a natural way. In the remaining part of this section we will deal with a symmetric fundamental form $\gamma_{i j}$ only, and denote it by $g_{i j}$.

With the help of the symmetric affine connection, we may define the tensor of non-metricity $Q_{i j}{ }^{k} \mathrm{by}^{44}$

$$
Q_{i j}{ }^{k}:=g^{k l} \nabla_{l} g_{i j}
$$

Then the following identity holds:

$$
\Gamma_{i j}^{k}=\left\{\begin{array}{l}
k \\
i j
\end{array}\right\}+K_{i j}^{k}+\frac{1}{2}\left(Q^{k}{ }_{i j}+Q_{j i}{ }^{k}-Q_{j}{ }^{k}{ }_{i}\right)
$$

where the contorsion tensor $K_{i j}{ }^{k}$, a linear combination of torsion $S_{i j}{ }^{k}$, is defined by ${ }^{45}$

$$
K_{i j}{ }^{k}:=S^{k}{ }_{j i}+S^{k}{ }_{i j}-S_{i j}{ }^{k}=-K_{i}{ }^{k}{ }_{j} .
$$

\footnotetext{
${ }^{42}$ This scalar density was used by Einstein [77] (cf. Section 6.4.4).

43 Tullio Levi-Civita (1873-1941). Born in Padua, Italy. Studied mathematics and received his doctorate at the University of Padua. Was given the Chair of Mechanics there and, in 1918, went to the University of Rome in the same position. Together with Ricci, he developed tensor calculus and introduced covariant differentiation. He worked also in the mechanical many-body problem, in hydrodynamics, general relativity theory, and unified field theory. Strongly opposed to Fascism in Italy and dismissed from his professorship in 1938.

${ }^{44}$ This definition differs from Schouten's by an overall minus sign.

${ }^{45}$ See [157]; his sign conventions are different, though.
} 
The inner product of two tangent vectors $A^{i}, B^{k}$ is not conserved under parallel transport of the vectors along $X^{l}$ if the non-metricity tensor does not vanish:

$$
X^{k} \stackrel{+}{\nabla}_{k}\left(A^{n} B^{m} g_{n m}\right)=Q_{n m l} A^{n} B^{m} X^{l} \neq 0 .
$$

A connection for which the non-metricity tensor vanishes, i.e.,

$$
\stackrel{+}{\nabla}_{k} g_{i j}=0
$$

holds, is called metric-compatible ${ }^{46}$.

J. M. Thomas ${ }^{47}$ introduced a combination of the terms appearing in $\stackrel{+}{\nabla}$ and $\bar{\nabla}$ to define a covariant derivative for the metric ([346], p. 188),

$$
g_{i k / l}:=g_{i k, l}-g_{r k} \Gamma_{i l}^{r}-g_{i r} \Gamma_{l k}^{r},
$$

and extended it for tensors of arbitrary rank $\geq 3$.

Einstein later used as a constraint on the metrical tensor

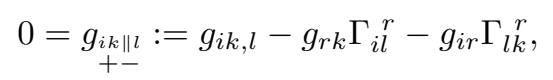

a condition that cannot easily be interpreted geometrically [97]. We will have to deal with Equation (47) in Section 6.1 and, more intensively, in Part II of this review.

Connections that are not metric-compatible have been used in unified field theory right from the beginning. Thus, in Weyl's theory [397, 395] we have

$$
Q_{i j k}=Q_{k} g_{i j}
$$

In case of such a relationship, the geometry is called semi-metrical [300, 310]. According to Equation (44), in Weyl's theory the inner product multiplies by a scalar factor under parallel transport:

$$
X^{k} \stackrel{+}{\nabla}_{k}\left(A^{n} B^{m} g_{n m}\right)=\left(Q_{l} X^{l}\right) A^{n} B^{m} g_{n m} .
$$

This means that the light cone is preserved by parallel transport.

We may also abbreviate the last term in the identity (42) by introducing

$$
X_{i j}{ }^{k}:=Q^{k}{ }_{i j}+Q_{j i}{ }^{k}-Q_{j}{ }^{k}{ }_{i}
$$

Then, from Equation (39), the curvature tensor of a torsionless affine space is given by

$$
K_{j k l}^{i}(\bar{\Gamma})=K_{j k l}^{i}\left(\left\{\begin{array}{c}
r \\
n m
\end{array}\right\}\right)+2^{\left(\left\{_{j k}^{i}\right\}\right)} \nabla_{[k} X_{l] j}^{i}-2 X_{[k|j|}^{m} X_{l] m}^{i},
$$

where ${ }^{\left(\left\{{ }_{j k}^{i}\right\}\right)} \nabla$ is the covariant derivative formed with the Christoffel symbol.

Riemann-Cartan geometry is the subcase of a metric-affine geometry in which the metriccompatible connection contains torsion, i.e., an antisymmetric part $L_{[i j]}^{k}$; torsion is a tensor field

\footnotetext{
${ }^{46}$ In the case of an asymmetric metric, this relation must be satisfied separately by the symmetric and antisymmetric parts of $g$ :

$$
\nabla_{k} h_{i j}=0, \quad \nabla_{k} \phi_{i j}=0 .
$$

47 Joseph Miller Thomas (1898-1979). Studied mathematics in Philadelphia at the University of Pennsylvania. Received his doctorate in 1923. From 1927 assistant professor at the University of Pennsylvania, from 1930 assistant and in 1935 full professor of mathematics at the Duke University in Durham, North Carolina. His fields were differential geometry and partial differential equations. He was the principle founder of Duke Mathematical Journal.
} 
to be linked to physical observables. A linear connection whose antisymmetric part $S_{i j}{ }^{k}$ has the form

is called semi-symmetric [300].

$$
S_{i j}{ }^{k}=S_{[i} \delta_{j]}^{k}
$$

Riemannian geometry is the further subcase with vanishing torsion of a metric-affine geometry with metric-compatible connection. In this case, the connection is derived from the metric: $\Gamma_{i j}{ }^{k}=$ $\left\{\begin{array}{l}k \\ i j\end{array}\right\}$, where $\left\{\begin{array}{c}k \\ i j\end{array}\right\}$ is the usual Christoffel symbol (40). The covariant derivative of $A$ with respect to the Levi-Civita connection $\stackrel{\left\{\begin{array}{l}k \\ i j\end{array}\right\}}{\nabla}$ is abbreviated by $A_{; k}$. The Riemann curvature tensor is denoted by

$$
R_{j k l}^{i}=\partial_{k}\left\{\begin{array}{l}
i \\
l j
\end{array}\right\}-\partial_{l}\left\{\begin{array}{c}
i \\
k j
\end{array}\right\}+\left\{\begin{array}{c}
i \\
k m
\end{array}\right\}\left\{\begin{array}{c}
m \\
l j
\end{array}\right\}-\left\{\begin{array}{c}
i \\
l m
\end{array}\right\}\left\{\begin{array}{c}
m \\
k j
\end{array}\right\} .
$$

An especially simple case of a Riemanian space is Minkowski space, the curvature of which vanishes:

$$
R_{j k l}^{i}(\eta)=0 .
$$

This is an invariant characterisation irrespective of whether the Minkowski metric $\eta$ is given in Cartesian coordinates as in Equation (10), or in an arbitrary coordinate system. We also have $\mathcal{L}_{X} R_{i j k l}=0$ where $\mathcal{L}$ is the Lie-derivative (see below under "symmetries"), and $X$ stands for the generators of the Lorentz group.

In Riemanian geometry, the so-called geodesic equation,

$$
X_{; k}^{i} X^{k}(u)=\sigma(u) X^{i}
$$

determines the shortest and the straightest curve between two infinitesimally close points. However, in metric affine and in mixed geometry geodesic and autoparallel curves will have to be distinguished.

A conformal transformation of the metric,

$$
g_{i k} \rightarrow g_{i k}^{\prime}=\lambda g_{i k},
$$

with a smooth function $\lambda$ changes the components of the non-metricity tensor,

$$
Q_{i j}{ }^{k} \rightarrow Q_{i j}{ }^{k}+g_{i j} g^{k l} \partial_{l} \sigma,
$$

as well as the Levi-Civita connection,

$$
\left\{\begin{array}{c}
k \\
i j
\end{array}\right\} \rightarrow\left\{\begin{array}{l}
k \\
i j
\end{array}\right\}+\frac{1}{2}\left(\sigma_{i} \delta_{j}^{k}-\sigma_{j} \delta_{i}^{k}+g_{i j} g^{k l} \sigma_{l}\right),
$$

with $\sigma_{i}:=\lambda^{-1} \partial_{i} \lambda$. As a consequence, the Riemann curvature tensor $R_{j k l}^{i}$ is also changed; if, however, $R^{i}{ }_{j k l}=0$ can be reached by a conformal transformation, then the corresponding spacetime is called conformally flat. In $M_{D}$, for $D>3$, the vanishing of the Weyl curvature tensor

$$
C_{j k l}^{i}:=R_{j k l}^{i}+\frac{2}{D-2}\left(\delta_{[k}^{i} R_{l] j}+g_{j[l} R_{k]}^{i}\right)+\frac{2 R}{(D-1)(D-2)} \delta_{\left[{ }_{l} g_{k] j}\right.}
$$

is a necessary and sufficient condition for $M_{D}$ to be conformally flat ([397], p. 404, [300], p. 170).

Even before Weyl, the question had been asked (and answered) as to what extent the conformal and the projective structures were determining the geometry: According to Kretschmann (and then to Weyl) they fix the metric up to a constant factor ([196]; see also [401], Appendix 1; for a modern approach, cf. [67]).

The geometry needed for the pre- and non-relativistic approaches to unified field theory will have to be dealt with separately. There, the metric tensor of space is Euclidean and not of full rank; time is described by help of a linear form (Newton-Cartan geometry, cf. [65, 66]). In the following we shall deal only with relativistic unified field theories. 
2.1.3.3 Projective geometry Projective geometry is a generalisation of Riemannian geometry in the following sense: Instead of tangent spaces with the light cone $\eta_{i k} d x^{i} d x^{k}=0$, where $\eta$ is the Minkowski metric, in each event now a tangent space with a general, non-degenerate surface of second order $\gamma$ will be introduced. This leads to a tangential cone $g_{i k} d x^{i} d x^{k}=0$ in the origin (cf. Equation (9)), and to a hyperplane, the polar plane, formed by the contact points of the tangential cone and the surface $\gamma$. In place of the $D$ inhomogeneous coordinates $x^{i}$ of $M_{D}$, $D+1$ homogeneous coordinates $X^{\alpha}(\alpha=0,1,2, \ldots, D)$ are defined ${ }^{48}$ such that they transform as homogeneous functions of first degree:

$$
X^{\alpha} \frac{\partial X^{\prime \nu}}{\partial X^{\mu}}=X^{\prime \nu}
$$

The connection to the inhomogeneous coordinates $x^{i}$ is given by homogeneous functions of degree zero, e.g., by $x^{i}=\frac{X^{i}}{\phi_{\alpha} X^{\alpha}}{ }^{49}$. Thus, the $X^{\alpha}$ themselves form the components of a tangent vector. Furthermore, the quadratic form $g_{\alpha \beta} X^{\alpha} X^{\beta}=\epsilon= \pm 1$ is adopted with $g_{\alpha \beta}$ being a homogeneous

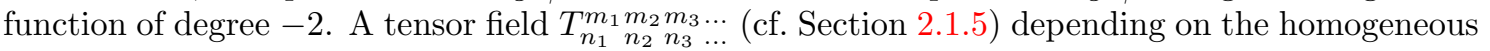
coordinates $X^{\mu}$ with $u$ contravariant (upper) and $l$ covariant (lower) indices is required to be a homogeneous function of degree $r:=u-l$.

If we define $\gamma_{\mu}^{i}:=\frac{\partial x^{i}}{\partial X^{\mu}}$, with $\gamma_{\mu}^{i} X^{\mu}=0$, then $\gamma_{\mu}^{i}$ transforms like a tangent vector under point transformations of the $x^{i}$, and as a covariant vector under homogeneous transformations of the $X^{\alpha}$. The $\gamma_{\mu}^{i}$ may be used to relate covariant vectors $a_{i}$ and $A_{\mu}$ by $A_{\mu}=\gamma_{\mu}^{i} a_{i}$. Thus, the metric tensor in the space of homogeneous coordinates $g_{\alpha \beta}$ and the metric tensor $g_{i k}$ of $M_{D}$ are related by $g_{i k}=$ $\gamma_{i}{ }^{\alpha} \gamma_{k}{ }^{\beta} g_{\alpha \beta}$ with $\gamma_{\mu}^{i} \gamma_{k}{ }^{\mu}=\delta_{k}^{i}$. The inverse relationship is given by $g_{\alpha \beta}=\gamma_{\alpha}^{i} \gamma_{\beta}^{k} g_{i k}+\epsilon X_{\alpha} X_{\beta}$ with $X_{\alpha}=g_{\alpha \beta} X^{\beta}$. The covariant derivative for tensor fields in the space of homogeneous coordinates is defined as before (cf. Section 2.1.2):

$$
\nabla_{\alpha} A^{\beta}(X)=\frac{\partial A^{\beta}(X)}{\partial X^{\alpha}}+\Gamma_{\alpha \nu}^{\beta}(X) A^{\nu}(X)
$$

The covariant derivative of the quantity $\gamma_{k}^{\mu}$ interconnecting both spaces is given by

$$
\nabla_{\rho} \gamma_{k}^{\mu}=\frac{\partial \gamma_{k}^{\mu}}{\partial x^{\rho}}+\Gamma_{\rho \sigma}^{\mu} \gamma_{k}^{\sigma}-\left\{\begin{array}{l}
m \\
k l
\end{array}\right\} \gamma_{\rho}^{l} \gamma_{m}^{\mu}
$$

\subsubsection{Cartan's method}

In this section, we briefly present Cartan's one-form formalism in order to make understandable part of the literature. Cartan introduces one-forms $\theta^{\hat{a}}(\hat{a}=1, \ldots, 4)$ by $\theta^{\hat{a}}:=h_{l}^{\hat{a}} d x^{l}$. The reciprocal basis in tangent space is given by $e_{\hat{\jmath}}=h_{\hat{\jmath}}^{l} \frac{\partial}{\partial x^{l}}$. Thus, $\theta^{\hat{a}}\left(e_{\hat{\jmath}}\right)=\delta_{\hat{\jmath}}^{\hat{a}}$. The metric is then given by $\eta_{\hat{\imath} \hat{k}} \theta^{\hat{\imath}} \otimes \theta^{\hat{k}}$. The covariant derivative of a tangent vector with bein-components $X^{\hat{k}}$ is defined via Cartan's first structure equations,

$$
\Theta^{i}:=D \theta^{\hat{\imath}}=d \theta^{\hat{\imath}}+\omega_{\hat{l}}^{\hat{\imath}} \wedge \theta^{\hat{l}},
$$

where $\omega_{\hat{k}}^{\hat{\imath}}$ is the connection-1-form, and $\Theta^{\hat{\imath}}$ is the torsion-2-form, $\Theta^{\hat{\imath}}=-S_{\hat{l} \hat{m}}{ }^{\hat{\imath}} \theta^{\hat{l}} \wedge \theta^{\hat{m}}$. We have $\omega_{\hat{\imath} \hat{k}}=-\omega_{\hat{k} \hat{\imath}}$. The link to the components $L_{[i j]}^{k}$ of the affine connection is given by $\omega_{\hat{k}}^{\hat{\imath}}=$ $h_{l}^{\hat{\imath}} h_{\hat{k}}^{m} L_{\hat{r} m}^{l} \theta^{\hat{r} 50}$. The covariant derivative of a tangent vector with bein-components $X^{\hat{k}}$ then is

$$
D X^{\hat{k}}:=d X^{\hat{k}}+\omega^{\hat{k}} X^{\hat{l}}
$$

\footnotetext{
${ }^{48}$ The index 0 does not refer to a time-coordinate.

${ }^{49}$ Note that $X^{\alpha}$ and $\lambda X^{\alpha}$ correspond to the same point of $M_{D}$; Veblen uses $\lambda=\exp \left(N x^{0}\right)$ (see [379], Section II)

${ }^{50}$ For an asymmetric connection, this corresponds to the + derivative.
} 
By further external derivation ${ }^{51}$ on $\Theta$ we arrive at the second structure relation of Cartan,

$$
D \Theta^{\hat{k}}=\Omega_{\hat{l}}^{\hat{k}} \wedge \theta^{\hat{l}}
$$

In Equation (65) the curvature-2-form $\Omega_{\hat{l}}^{\hat{k}}=\frac{1}{2} R_{\hat{l} \hat{m} \hat{n}}^{\hat{k}} \theta^{\hat{m}} \wedge \theta^{\hat{n}}$ appears, which is given by

$$
\Omega_{\hat{l}}^{\hat{k}}=d \omega_{\hat{l}}^{\hat{k}}+\omega_{\hat{l}}^{\hat{k}} \wedge \omega_{\hat{l}}^{\hat{k}}
$$

$\Omega_{\hat{k}}^{\hat{k}}$ is the homothetic curvature.

\subsubsection{Tensors, spinors, symmetries}

2.1.5.1 Tensors Up to here, no definitions of a tensor and a tensor field were given: A tensor $T_{p}\left(M_{D}\right)$ of type $(r, s)$ at a point $p$ on the manifold $M_{D}$ is a multi-linear function on the Cartesian product of $r$ cotangent- and $s$ tangent spaces in $p$. A tensor field is the assignment of a tensor to each point of $M_{D}$. Usually, this definition is stated as a linear, homogeneous transformation law for the tensor components in local coordinates:

$$
T_{l_{1}^{\prime} l_{2}^{\prime} l_{3}^{\prime} \ldots}^{k_{1}^{\prime} k_{1}^{\prime} k_{3}^{\prime} \ldots}=T_{n_{1} n_{2} n_{3} \ldots}^{m_{1} m_{2} m_{3} \ldots} \frac{\partial x^{n_{1}}}{\partial x^{l_{1}^{\prime}}} \frac{\partial x^{n_{2}}}{\partial x^{l_{2}^{\prime}}} \frac{\partial x^{n_{3}}}{\partial x^{l_{3}^{\prime}}} \frac{\partial x^{k_{1}^{\prime}}}{\partial x^{m_{1}}} \frac{\partial x^{k_{2}^{\prime}}}{\partial x^{m_{2}}} \frac{\partial x^{k_{3}^{\prime}}}{\partial x^{m_{3}}} \cdots
$$

where $x^{k^{\prime}}=x^{k^{\prime}}\left(x^{i}\right)$ with smooth functions on the r.h.s. are taken from the set ("group") of coordinate transformations (diffeomorphisms). Strictly speaking, tensors are representations of the abstract group at a point on the manifold ${ }^{52}$.

A relative tensor $T_{p}\left(M_{D}\right)$ of type $(r, s)$ and of weight $\omega$ at a point $p$ on the manifold $M_{D}$ transforms like

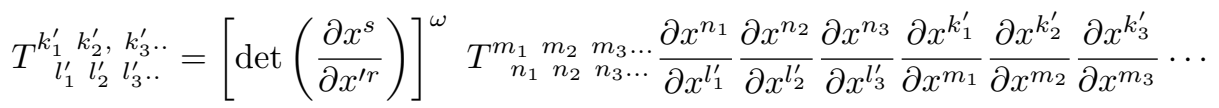

An example is given by the totally antisymmetric object $\epsilon_{i j k l}$ with $\epsilon_{i j k l}= \pm 1$, or $\epsilon_{i j k l}=0$ depending on whether $(i j k l)$ is an even or odd permutation of $(0123)$, or whether two indices are alike. $\omega=-1$ for $\epsilon_{i j k l}$; in this case, the relative tensor is called tensor density. We can form a tensor from $\epsilon_{i j k l}$ by introducing $\eta_{i j k l}:=\sqrt{-g} \epsilon_{i j k l}$, where $g_{i k}$ is a Lorentz-metric. Note that $\eta^{i j k l}:=\frac{1}{\sqrt{-g}} \epsilon^{i j k l}$. The dual to a 2 -form (skew-symmetric tensor) then is defined by ${ }^{*} F^{i j}=\frac{1}{2} \eta^{i j k l} F_{k l}$.

In connection with conformal transformations $g \rightarrow \lambda g$, the concept of the gauge-weight of a tensor is introduced. A tensor $T^{\cdots} \ldots$ is said to be of gauge weight $q$ if it transforms by Equation (56) as

$$
T^{\prime \cdots}=\lambda^{q} T^{\ldots}
$$

Objects that transform as in Equation (67) but with respect to a subgroup, e.g., the linear group, affine group $G(D)$, orthonormal group $O(D)$, or the Lorentz group $\mathcal{L}$, are tensors in a restricted sense; sometimes they are named affine or Cartesian tensors. All the subgroups mentioned are Lie-groups, i.e., continuous groups with a finite number of parameters. In general relativity, both

\footnotetext{
${ }^{51}$ The external derivative $d$ of linear forms $\omega, \mu$ satisfies the following rules:

(1) $d(a \omega+b \mu)=a d \omega+b d \mu$,

(2) $d(\omega \wedge \mu)=d \omega \wedge \mu-\omega \wedge d \mu$

(3) $d d \omega=0$.

${ }^{52} \mathrm{~A}$ representation of a group is defined as a map to the vectors of a linear space that is homomorphic in the group operation.
} 
the "group" of general coordinate transformations and the Lorentz group are present. The concept of tensors used in Special Relativity is restricted to a representation of the Lorentz group; however, as soon as the theory is to be given a coordinate-independent ("generally covariant") form, then the full tensor concept comes into play.

2.1.5.2 Spinors Spinors are representations of the Lorentz group only; as such they are related strictly to the tangent space of the space-time manifold. To see how spinor representations can be obtained, we must use the 2-1 homomorphism of the group $S L(2, \mathbf{C})$ and the proper orthochronous Lorentz group, a subgroup of the full Lorentz group ${ }^{53}$. Let $\mathbf{A} \in S L(2, \mathbf{C})$; then $\mathbf{A}$ is a complex (2-by-2)-matrix with $\operatorname{det} \mathbf{A}=1$. By picking the special Hermitian matrix

$$
\mathbf{S}=x^{0} \mathbf{1}+\sum_{p} \sigma_{p} x^{p}
$$

where $\mathbf{1}$ is the (2 by 2)-unit matrix and $\sigma_{p}$ are the Pauli matrices satisfying

$$
\sigma_{i} \sigma_{k}+\sigma_{k} \sigma_{i}=2 \delta_{i k}
$$

Then, by a transformation A from $S L(2, \mathbf{C})$,

$$
\mathbf{S}^{\prime}=\mathbf{A S A}^{+}
$$

where $\mathbf{A}^{+}$is the Hermitian conjugate matrix ${ }^{54}$. Moreover, $\operatorname{det} \mathbf{S}=\operatorname{det} \mathbf{S}^{\prime}$ which, according to Equation (70), expresses the invariance of the space-time distance to the origin:

$$
\left(x^{0^{\prime}}\right)^{2}-\left(x^{1^{\prime}}\right)^{2}-\left(x^{2^{\prime}}\right)^{2}-\left(x^{3^{\prime}}\right)^{2}=\left(x^{0}\right)^{2}-\left(x^{1}\right)^{2}-\left(x^{2}\right)^{2}-\left(x^{3}\right)^{2} .
$$

The link between the representation of a Lorentz transformation $L_{i k}$ in space-time and the unimodular matrix A mapping spin space (cf. below) is given by

$$
L(\mathbf{A})_{i k}=\frac{1}{2} \operatorname{tr}\left(\sigma_{i} \mathbf{A} \sigma_{k} \mathbf{A}^{+}\right) .
$$

Thus, the map is two to one: $+\mathbf{A}$ and $-\mathbf{A}$ give the same $L_{i k}$.

Now, contravariant 2-spinors $\xi^{A}(A=1,2)$ are the elements of a complex linear space, spinor space, on which the matrices $\mathbf{A}$ are acting ${ }^{55}$. The spinor is called elementary if it transforms under a Lorentz-transformation as

$$
\xi^{A^{\prime}}= \pm \mathbf{A}_{C}^{A} \xi^{C}
$$

Likewise, contravariant dotted spinors $\zeta^{\dot{A}}$ are those transforming with the complex-conjugate ma$\operatorname{trix} \overline{\mathbf{A}}$ :

$$
\zeta^{\dot{B}^{\prime}}= \pm \overline{\mathbf{A}}_{\dot{D}}^{\dot{B}} \zeta^{\dot{C}}
$$

Covariant and covariant dotted 2-spinors correspondingly transform with the inverse matrices,

$$
\xi_{B^{\prime}}= \pm\left(\mathbf{A}^{-1}\right)_{B}^{C} \xi_{C}
$$

and

$$
\xi_{\dot{B}^{\prime}}= \pm\left(\overline{\mathbf{A}}^{-1}\right)_{\dot{B}}^{\dot{C}} \xi_{\dot{C}}
$$

The space of 2-spinors can be used as a representation space for the (proper, orthochronous) Lorentz group, with the 2 -spinors being the elements of the most simple representation $D^{(1 / 2,0)}$.

\footnotetext{
${ }^{53}$ The full Lorentz group contains as further elements the temporal and spatial reflections.

${ }^{54} \mathbf{X}^{+}{ }_{A B}=\mathbf{X}_{B A} ; A, B=1,2$.

${ }^{55} 2$-component spinors are also called Weyl-spinors.
} 
Higher-order spinors with dotted and undotted indices $S_{C \ldots \dot{B} \ldots}^{A \ldots}$ transform correspondingly. For the raising and lowering of indices now a real, antisymmetric $(2 \times 2)$-matrix $\epsilon$ with components $\epsilon^{A B}=\delta_{1}^{A} \delta_{B}^{2}-\delta_{2}^{A} \delta_{B}^{1}=\epsilon_{A B}$ is needed, such that

$$
\xi^{A}=\epsilon^{A B} \xi_{B}, \quad \xi_{A}=\xi^{B} \epsilon_{B A}
$$

Next to a spinor, bispinors of the form $\zeta^{A B}, \xi^{A \dot{B}}$, etc. are the simplest quantities (spinors of 2nd order). A vector $X^{k}$ can be represented by a bispinor $X^{A \dot{B}}$,

$$
X^{A \dot{B}}=\sigma_{k}^{A \dot{B}} X^{k},
$$

where $\sigma_{k}^{A \dot{B}}(k=0, \ldots, 3)$ is a quantity linking the tangent space of space-time and spinor space. If $k$ numerates the matrices and $A, \dot{B}$ designate rows and columns, then we can chose $\sigma_{0}^{A \dot{B}}$ to be the unit matrix while for the other three indices $\sigma_{j}^{A \dot{B}}$ are taken to be the Pauli matrices. Often the quantity $s_{k}^{A \dot{B}}=\frac{1}{\sqrt{2}} \sigma_{k}^{A \dot{B}}$ is introduced. The reciprocal matrix $s_{A \dot{B}}^{k}$ is defined by

$$
s_{j}^{A \dot{B}} s_{A \dot{B}}^{k}=\delta_{j}^{k},
$$

whereas

$$
s_{j}^{A \dot{B}} s^{j C \dot{D}}=\epsilon^{A C} \epsilon^{\dot{B} \dot{D}} .
$$

In order to write down spinorial field equations, we need a spinorial derivative,

$$
\partial_{A \dot{B}}=s_{A \dot{B}}^{k} \partial_{k}
$$

with $\partial_{A \dot{B}} \partial^{A \dot{B}}=\partial_{k} \partial^{k}$. The simplest spinorial equation is the Weyl equation:

$$
\partial_{A \dot{B}} \psi^{A}=0, \quad \dot{B}=1,2 .
$$

The next simplest spinor equation for two spinors $\chi^{\dot{B}}, \psi_{A}$ would be

$$
\partial_{A \dot{C}} \chi^{\dot{C}}=-\frac{2 \pi}{\sqrt{2} h} m \psi_{A} ; \quad \partial^{C \dot{B}} \psi_{C}=\frac{2 \pi}{\sqrt{2} h} m \chi^{\dot{B}},
$$

where $m$ is a mass. Equation (85) is the 2-spinor version of Dirac's equation.

Dirac- or 4-spinors with 4 components $\psi^{k}, k=1, \ldots, 4$, may be constructed from 2-spinors as a direct sum of contravariant undotted and covariant dotted spinors $\psi$ and $\phi$ : For $k=1,2$, we enter $\psi^{1}$ and $\psi^{2}$; for $k=3,4$, we enter $\phi_{1}$ and $\phi_{\dot{2}}$. In connection with Dirac spinors, instead of the Pauli-matrices the Dirac $\gamma$-matrices $(4 \times 4$-matrices $)$ appear; they satisfy

$$
\gamma^{i} \gamma^{k}+\gamma^{k} \gamma^{i}=2 \eta^{i k} \mathbf{1}
$$

The Dirac equation is in 4-spinor formalism [53, 54]:

$$
\left(i \gamma^{l} \frac{\partial}{\partial x^{l}}+\kappa\right) \chi=0
$$

with the 4-component Dirac spinor $\chi$. In the first version of Dirac's equation, $\alpha$ - and $\beta$-matrices were used, related to the $\gamma$ 's by

$$
\gamma^{0}=\beta, \quad \gamma^{m}=\beta \alpha^{m}, \quad m=1,2,3,
$$

where the matrices $\beta$ and $\alpha^{m}$ are given by $\left(\begin{array}{cc}\mathbf{0} & -\sigma_{i} \\ \sigma_{i} & \mathbf{0}\end{array}\right),\left(\begin{array}{cc}\mathbf{0} & -\mathbf{1} \\ \mathbf{1} & \mathbf{0}\end{array}\right)$. 
The generally-covariant formulation of spinor equations necessitates the use of $n$-beins $h_{\hat{\imath}}^{k}$, whose internal "rotation" group, operating on the "hatted" indices, is the Lorentz group. The group of coordinate transformations acts on the Latin indices. In Cartan's one-form formalism (cf. Section 2.1.4), the covariant derivative of a 4 -spinor is defined by

$$
D \psi=d \psi+\frac{1}{4} \omega_{\hat{\imath} \hat{k}} \sigma^{\hat{\imath} \hat{k}} \psi
$$

where $\sigma^{\hat{\imath} \hat{k}}:=\frac{1}{2}\left[\gamma^{\hat{\imath}} \gamma^{\hat{k}}\right]$.

Equation (89) is a special case of the general formula for the covariant derivative of a tensorial form $\psi$, i.e., a vector in some vector space $V$, whose components are differential forms,

$$
D \psi=d \psi+\omega^{\hat{\imath} \hat{k}} \rho_{\hat{\imath} \hat{k}}\left(e_{\alpha}\right) \psi,
$$

where $\rho\left(e_{\alpha}\right)$ is a particular representation of the corresponding Lie algebra in $V$ with basis vectors $e_{\alpha}$. For the example of the Dirac spinor, the adjoint representation of the Lorentz group must be used $^{56}$.

2.1.5.3 Symmetries In Section 2.1.1 we briefly met the Lie derivative of a vector field $\mathcal{L}_{X}$ with respect to the tangent vector $X$ defined by $\left(L_{X} Y\right)^{k}:=([X, Y])^{k}=X^{i} \partial_{i} Y^{k}-Y^{i} \partial_{i} X^{k}$. With its help we may formulate the concept of isometries of a manifold, i.e., special mappings, also called "motions", locally generated by vector fields $X$ satisfying

$$
\mathcal{L}_{X} g_{i k}:=\partial_{k}\left(g_{i j}\right) X^{k}+g_{l j} \partial_{i} X^{l}+g_{i l} \partial_{j} X^{l}=0 .
$$

The generators $X$ solving Equation (91) given some metric, form a Lie group $G_{r}$, the group of motions of $M_{D}$. If a group $G_{r}$ is prescribed, e.g., the group of spatial rotations $O(3)$, then from Equation (91) the functional form of the metric tensor having $O(3)$ as a symmetry group follows.

A Riemannian space is called (locally) stationary if it admits a timelike Killing vector; it is called (locally) static if this Killing vector is hypersurface orthogonal. Thus if, in a special coordinate system, we take $X^{i}=\delta_{0}^{i}$ then from Equation (91) we conclude that stationarity reduces to the condition $\partial_{0} g_{i k}=0$. If we take $X$ to be the tangent vector field to the congruence of curves $x^{i}=x^{i}(u)$, i.e., if $X^{k}=\frac{d x^{k}}{d u}$, then a necessary and sufficient condition for hypersurfaceorthogonality is $\epsilon^{i j k l} X_{j} X_{[k, l]}=0$.

A generalisation of Killing vectors are conformal Killing vectors for which $\mathcal{L}_{X} g_{i k}=\Phi g_{i k}$ with an arbitrary smooth function $\Phi$ holds. In purely affine spaces, another type of symmetry may be defined: $\mathcal{L}_{X} \Gamma_{i k}^{l}=0$; they are called affine motions [425].

\subsection{Dynamics}

Within a particular geometry, usually various options for the dynamics of the fields (field equations, in particular as following from a Lagrangian) exist as well as different possibilities for the identification of physical observables with the mathematical objects of the formalism. Thus, in general relativity, the field equations are derived from the Lagrangian

$$
\mathcal{L}=\sqrt{-g}\left(R+2 \Lambda-2 \kappa L_{\mathrm{M}}\right)
$$

where $R\left(g_{i k}\right)$ is the Ricci scalar, $g:=\operatorname{det} g_{i k}, \Lambda$ the cosmological constant, and $L_{\mathrm{M}}$ the matter Lagrangian depending on the metric, its first derivatives, and the matter variables. This Lagrangian leads to the well-known field equations of general relativity,

$$
R^{i k}-\frac{1}{2} R g^{i k}=-\kappa T^{i k}
$$

\footnotetext{
${ }^{56}$ For a contemporary exposition of the use of spinors in space-time, see the book of Penrose and Rindler [256].
} 
with the energy-momentum(-stress) tensor of matter

$$
T^{i k}:=\frac{2}{\sqrt{-g}} \frac{\delta\left(\sqrt{-g} L_{\mathrm{M}}\right)}{\delta g_{i k}}
$$

and $\kappa=\frac{8 \pi G}{c^{4}}$, where $G$ is Newton's gravitational constant. $G^{i k}:=R^{i k}-\frac{1}{2} R g^{i k}$ is called the Einstein tensor. In empty space, i.e., for $T^{i k}=0$, Equation (92) reduces to

$$
R^{i k}=0
$$

If only an electromagnetic field $F_{i k}=\frac{\partial A_{k}}{\partial x^{i}}-\frac{\partial A_{i}}{\partial x^{k}}$ derived from the 4-vector potential $A_{k}$ is present in the energy-momentum tensor, then the Einstein-Maxwell equations follow:

$$
R^{i k}-\frac{1}{2} R g^{i k}=-\kappa\left(F_{i l} F_{k}^{l}+\frac{1}{4} g_{i k} F_{l m} F^{l m}\right), \quad \nabla_{l} F^{i l}=0 .
$$

The components of the metrical tensor are identified with gravitational potentials. Consequently, the components of the (Levi-Civita) connection correspond to the gravitational "field strength", and the components of the curvature tensor to the gradients of the gravitational field. The equations of motion of material particles should follow, in principle, from Equation (92) through the relation

$$
\nabla_{l} T^{i l}=0
$$

implied by $\mathrm{it}^{57}$. For point particles, due to the singularities appearing, in general this is a tricky task, up to now solved only approximately. However, the world lines for point particles falling freely in the gravitational field are, by definition, the geodesics of the Riemannian metric. This definition is consistent with the rigourous derivation of the geodesic equation for non-interacting dust particles in a fluid matter description. It is also consistent with all observations.

For most of the unified field theories to be discussed in the following, such identifications were made on internal, structural reasons, as no link-up to empirical data was possible. Due to the inherent wealth of constructive possibilities, unified field theory never would have come off the ground proper as a physical theory even if all the necessary formal requirements could have been satisfied. As an example, we take the identification of the electromagnetic field tensor with either the skew part of the metric, in a "mixed geometry" with metric compatible connection, or the skew part of the Ricci tensor in metric-affine theory, to list only two possibilities. The latter choice obtains likewise in a purely affine theory in which the metric is a derived secondary concept. In this case, among the many possible choices for the metric, one may take it proportional to the variational derivative of the Lagrangian with respect to the symmetric part of the Ricci tensor. This does neither guarantee the proper signature of the metric nor its full rank. Several identifications for the electromagnetic 4-potential and the electric current vector density have also been suggested (cf. below and [143]).

\subsection{Number field}

Complex fields may also be introduced on a real manifold. Such fields have also been used for the construction of unified field theories, although mostly after the period dealt with here (cf. Part II, in preparation). In particular, manifolds with a complex fundamental form were studied, e.g., with $g_{i k}=s_{i k}+i a_{i k}$, where $i=\sqrt{-1}$ [97]. Also, geometries based on Hermitian forms were

\footnotetext{
${ }^{57}$ In the early papers on general relativity, Equation (96) was called a "conservation law" because, in Minkowski space, it implies the conservation laws for energy and linear momentum. For an arbitrary Riemannian manifold this no longer holds true.
} 
studied [313]. In later periods, hypercomplex numbers, quaternions, and octonions also were used as basic number fields for gravitational or unified theories (cf. Part II, forthcoming).

In place of the real numbers, by which the concept of manifold has been defined so far, we could take other number fields and thus arrive, e.g., at complex manifolds and so on. In this part of the article we do not need to take into account this generalisation.

\subsection{Dimension}

Since the suggestions by Nordström and Kaluza [238, 181], manifolds with $D>4$ have been used for unified field theories. In most of the cases, the additional dimensions were taken to be spacelike; nevertheless, manifolds with more than one direction of time also have been studied. 


\section{Early Attempts at a Unified Field Theory}

\subsection{First steps in the development of unified field theories}

Even before (or simultaneously with) the introduction and generalisation of the concept of parallel transport and covariant derivative by Hessenberg (1916/17) [160], Levi-Civita (1917), [204], Schouten (1918) [294], Weyl (1918) [397], and König (1919) [193], the introduction of an asymmetric metric was suggested by Rudolf Förster ${ }^{58}$ in 1917. In his letter to Einstein of 11 November 1917, he writes ([321], Doc. 398, p. 552):

"Perhaps, there exists a covariant 6-vector by which the appearance of electricity is explained and which springs lightly from the $g_{\mu \nu}$, not forced into it as an alien element." 59

Einstein replied:

"The aim of dealing with gravitation and electricity on the same footing by reducing both groups of phenomena to $g_{\mu \nu}$ has already caused me many disappointments. Perhaps, you are luckier in the search. I am totally convinced that in the end all field quantities will look alike in essence. But it is easier to suspect something than to discover it." 60 (16 November 1917 [321], Vol. 8A, Doc. 400, p. 557)

In his next letter, Förster gave results of his calculations with an asymmetric $g_{\mu \nu}=s_{\mu \nu}+a_{\mu \nu}$, introduced an asymmetric "three-index-symbol" and a possible generalisation of the Riemannian curvature tensor as well as tentative Maxwell's equations and interpretations for the 4-potential $A_{\mu}$, and special solutions (28 December 1917) ([321], Volume 8A, Document 420, pp. 581-587). Einstein's next letter of 17 January 1918 is skeptical:

"Since long, I also was busy by starting from a non-symmetric $g_{\mu \nu}$; however, I lost hope to get behind the secret of unity (gravitation, electromagnetism) in this way. Various reasons instilled in me strong reservations: [...] your other remarks are interesting in themselves and new to me." 61 ([321], Volume 8B, Document 439, pp. 610-611)

Einstein's remarks concerning his previous efforts must be seen under the aspect of some attempts at formulating a unified field theory of matter by G. Mie [229, 230, 231] ${ }^{62}$, J. Ishiwara, and G. Nordström, and in view of the unified field theory of gravitation and electromagnetism proposed by David Hilbert.

"According to a general mathematical theorem, the electromagnetic equations (generalized Maxwell equations) appear as a consequence of the gravitational equations, such that gravitation and electrodynamics are not really different." 63 (letter of Hilbert to Einstein of 13 November 1915 [162])

\footnotetext{
${ }^{58}$ Förster published under a nom de plume "R. Bach" because his employer Krupp did not like his employees using their free time on something as academical as research in gravitation and unified field theory. Bach wrote also about Weyl's theory (Bach 1921) [4].

59 "Vielleicht findet sich ein kovarianter Sechservektor der das Auftreten der Elektrizität erklärt und ungezwungen aus den $g_{\mu \nu}$ herauskommt, nicht als fremdes Element herangetragen wird."

60 "Das Ziel, Gravitation und Elektromagnetismus einheitlich zu behandeln, indem man beide Phänomengruppen auf die $g_{\mu \nu}$ zurückführt, hat mir schon viele erfolglose Bemühungen gekostet. Vielleicht sind Sie glücklicher im Suchen. Ich bin fest überzeugt, dass letzten Endes alle Feldgrössen sich als wesensgleich herausstellen werden. Aber leichter ist ahnen als finden."

61 "Das Ausgehen von einem nichtsymmetrischen $g_{\mu \nu}$ hat mich auch schon lange beschäftigt; ich habe aber die Hoffnung aufgegeben, auf diese Weise hinter das Geheimnis der Einheit (Gravitation-Elektromagnetismus) zu kommen. Verschiedene Gründe flössen da schwere Bedenken ein: [...] Ihre übrigen Bemerkungen sind ebenfalls an sich interessant und mir neu."

${ }^{62}$ In his textbook, M. von Laue presented and discussed Mie's theory [387].

63 "In Folge eines allgem. math. Satzes erscheinen die elektrody. Gl. (verallgemeinerte Maxwellsche) als math. Folge der Gravitationsgl., so dass Gravitation und Elektrodynamik eigentlich garnicht verschiedenes sind."
} 
The result is contained in (Hilbert 1915, p. 397) ${ }^{64}$.

Einstein's answer to Hilbert on 15 November 1915 shows that he had also been busy along such lines:

"Your investigation is of great interest to me because I have often tortured my mind in order to bridge the gap between gravitation and electromagnetism. The hints dropped by you on your postcards bring me to expect the greatest." ${ }^{65}$ [101]

Even before Förster alias Bach corresponded with Einstein, a very early bird in the attempt at unifying gravitation and electromagnetism had published two papers in 1917, Reichenbächer [270, 269]. His paper amounts to a scalar theory of gravitation with field equation $R=0$ instead of Einstein's $R_{a b}=0$ outside the electrons. The electron is considered as an extended body in the sense of Lorentz-Poincaré, and described by a metric joined continuously to the outside metric ${ }^{66}$ :

$$
d s^{2}=d r^{2}+r^{2} d \phi^{2}+r^{2} \cos ^{2} \phi d \psi^{2}+\left(1-\frac{\alpha}{r}\right)^{2} d x_{0}^{2}
$$

Reichenbächer, at this point, seems to have had a limited understanding of general relativity: He thinks in terms of a variable velocity of light; he equates coordinate systems and reference systems, and apparently considers the transition from the Minkowskian to a non-flat metric as achieved by a coordinate rotation, a "Drehung gegen den Normalzustand" ("rotation with respect to the normal state") ([270], p. 137). According to him, the deviation from the Minkowski metric is due to the electromagnetic field tensor:

"The disturbance, which is generated by the electrons and which forces us to adopt a coordinate system different from the usual one, is interpreted as the electromagnetic six-vector, as is known." 67 ([270], p. 136)

By his "coordinate rotation", or, as he calls it in ([269], p. 174), "electromagnetic rotation", he tries to geometrize the electromagnetic field. As Weyl's remark in Raum-Zeit-Materie ([398], p. 267, footnote 30) shows, he did not grasp Reichenbächer's reasoning; I have not yet understood it either. Apparently, for Reichenbächer the metric deviation from Minkowski space is due solely to the electromagnetic field, whereas gravitation comes in by a single scalar potential connected to the velocity of light. He claims to obtain the same value for the perihelion shift of Mercury as Einstein ([269], p. 177). Reichenbächer was slow to fully accept general relativity; as late as in 1920 he had an exchange with Einstein on the foundations of general relativity [271, 71].

After Reichenbächer had submitted his paper to Annalen der Physik and seemingly referred to Einstein,

"Planck was uncertain to which of Einstein's papers Reichenbächer appealed. He urged that Reichenbächer speak with Einstein and so dissolve their differences. The meeting was amicable. Reichenbächer's paper appeared in 1917 as the first attempt at a unified field theory in the wake of Einstein's covariant field equations." ([262], p. 208)

\footnotetext{
${ }^{64}$ See also the Diploma thesis by König [191]. In it it is shown that from the divergence relation $T^{\mu \nu}{ }_{i \nu}=0$ and the most general Lagrangian $L(u, v)$ with $u=F_{\mu \nu} F^{\mu \nu} a n d v={ }^{*} F_{\mu \nu} F^{\mu \nu}$, the field equations follow only for the generic case of full rank of the electromagnetic field tensor $F_{\mu \nu}$.

65 "Ihre Untersuchung interessiert mich gewaltig, zumal ich mir oft schon das Gehirn zermartert habe, um eine Brücke zwischen Gravitation und Elektromagnetik zu schlagen. Die Andeutungen, welche Sie auf Ihren Karten geben, lassen das Grösste erwarten."

${ }^{66}$ Reichenbächer's solution is a special case of a huge number of spherically symmetric solutions of $R=0$ given in Goenner and Havas 1980 [144]. Reichenbächer published 29 papers between 1917 and 1930.

67 "Die Störung, die durch die Elektronen erzeugt wird und uns also zur Annahme eines von dem gewöhnlichen abweichenden Weltkoordinatensystem zwingt, wird nun bekanntlich als der elektromagnetische Sechservektor aufgefasst."
}

Living Reviews in Relativity

http://www. livingreviews.org//rr-2004-2 
In this context, we must also keep in mind that the generalisation of the metric tensor toward asymmetry or complex values was more or less synchronous with the development of Finsler geometry [126]. Although Finsler himself did not apply his geometry to physics it soon became used in attempts at the unification of gravitation and electromagnetism [274].

\subsection{Early disagreement about how to explain elementary particles by field theory}

In his book on Einstein's relativity, Max Born, in 1920, had asked about the forces hindering "an electron or an atom" to disintegrate.

"Now, these objects are tremendous concentrations of energy in the smallest place; therefore, they will house huge curvatures of space or, in other words, gravitational fields. The idea that they keep together the dispersing electrical charges lies close at hand." 68 ([19], p. 235)

Thus, the idea of a program for building the extended constituents of matter from the fields the source of which they are, was very much alive around 1920. However, Pauli's remark after Weyl's lecture in Bad Nauheim (86. Naturforscherversammlung, 19-25 September 1920) [245] showed that not everybody was a believer in it. He claimed that in bodies smaller than those carrying the elementary charge (electrons), an electric field could not be measured. There was no point of creating the "interior" of such bodies with the help of an electric field. Pauli:

"None of the present theories of the electron, also not Einstein's (Einstein 1919 [70]), up to now did achieve solving satisfactorily the problem of the electrical elementary quanta; it seems obvious to look for a deeper reason for this failure. I wish to see this reason in the fact that it is altogether not permitted to describe the electromagnetic field in the interior of an electron as a continuous space function. The electrical field is defined as the force on a charged test particle, and if no smaller test particles exist than the electron (vice versa the nucleus), the concept of electrical field at a certain point in the interior of the electron - with which all continuum theories are working - seems to be an empty fiction, because there are no arbitrarily small measures. Therefore, I'd like to ask Mr. Einstein whether he approves of the opinion that a solution of the problem of matter may be expected only from a modification of our perception of space (perhaps also of time) and of electricity in the sense of atomism, or whether he thinks that the mentioned reservations are unconvincing and is of the opinion that the fundaments of continuum theory must be upheld." 69

\footnotetext{
68 "Nun sind diese Gebilde ungeheure Anhäufungen von Energie auf kleinsten Räumen; daher werden sie gewaltige Raumkrümmungen, oder mit anderen Worten, Gravitationsfelder, in sich bergen. Der Gedanke liegt nahe, dass diese es sind, die die auseinanderstrebenden elektrischen Ladungen zusammenhalten."

69 "Keiner der bisherigen Theorien des Elektrons, auch nicht der Einsteinschen (Einstein 1919 [70]) ist es bisher gelungen, das Problem der elektrischen Elementarquante befriedigend zu lösen, und es liegt nahe, nach einem tieferen Grund für diesen Mißerfolg zu suchen. Ich möchte nun diesen Grund darin suchen, daß es überhaupt unstatthaft ist, das elektrische Feld im Innern des Elektrons als stetige Raumfunktion zu beschreiben. Die elektrische Feldstärke ist definiert als die Kraft auf einen geladenen Probekörper und, wenn es keine kleineren Probekörper gibt als das Elektron (bzw den N-Kern), scheint der Begriff der elektrischen Feldstärke in einem bestimmten Punkt im Innern des Elektrons, mit welchem alle Kontinuumstheorien operieren, eine leere, inhaltslose Fiktion zu sein, da es keine beliebig kleinen Maßstäbe gibt. Ich möchte deshalb Herrn Einstein fragen, ob er der Auffassung zustimmt, daß man die Lösung des Problems der Materie nur von einer Modifikation unserer Vorstellungen vom Raum (vielleicht auch von der Zeit) und vom elektrischen Felde im Sinne des Atomismus erwarten darf, oder ob er die angeführten Bedenken nicht für stichhaltig hält und die Ansicht vertritt, daß man an den Grundlagen der Kontinuumstheorie festhalten muß."
} 
Pauli referred to Einstein's paper about elementary particles and field theory in which he had exchanged his famous field equations for traceless equations with the electromagnetic field tensor as a source. Einstein's answer is tentative and evasive: We just don't know yet ${ }^{70}$.

"With the progressing refinement of scientific concepts, the manner by which concepts are related to (physical) events becomes ever more complicated. If, in a certain stage of scientific investigation, it is seen that a concept can no longer be linked with a certain event, there is a choice to let the concept go, or to keep it; in the latter case, we are forced to replace the system of relations among concepts and events by a more complicated one. The same alternative obtains with respect to the concepts of timeand space-distances. In my opinion, an answer can be given only under the aspect of feasibility; the outcome appears dubious to me." 71

In the same discussion Gustav Mie came back to Förster's idea of an asymmetric metric but did not like it

"[...] that an antisymmetric tensor was added to the symmetric tensor of the gravitational potential, which represented the six-vector of the electromagnetic field. But a more precise reasoning shows that in this way no reasonable world function is obtained." 72

It is to be noted that Weyl, at the end of 1920, already had given up on a possible field theory of matter:

"Finally I cut loose firmly from Mie's theory and arrived at another position with regard to the problem of matter. To me, field physics no longer appears as the key to reality; in contrary, the field, the ether, for me simply is the totally powerless transmitter of causations, yet matter is a reality beyond the field and causes its states." 73 (letter of Weyl to F. Klein on 28 December 1920, see [293], p. 83)

In the next year, Einstein had partially absorbed Pauli's view but still thought it to be useful to apply field theory to the constituents of matter:

"The physical interpretation of geometry (theory of the continuum) presented here, fails in its direct application to spaces of submolecular scale. Yet it retains part of its meaning also with regard to questions concerning the constitution of elementary particles. Because one may try to ascribe to these field concepts [...] a physical meaning even if a description of the electrical elementary particles which constitute matter is

\footnotetext{
${ }^{70}$ For the evolution of Einstein's position vis-a-vis continuum theory and short-comings of it, cf. J. Stachel [329].

71 "Mit fortschreitender Verfeinerung des wissenschaftlichen Begriffssystems wird die Art und Weise der Zuordnung der Begriffe von den Erlebnissen immer komplizierter. Hat man in einem gewissen Studium der Wissenschaft gesehen, daß einem Begriff ein bestimmtes Erlebnis nicht mehr zugeordnet werden kann, so hat man die Wahl, ob man den Begriff fallen lassen oder ihn beibehalten will; in letzterem Fall ist man aber gezwungen, das System der Zuordnung der Begriffe zu den Erlebnissen durch ein komplizierteres zu ersetzen. Vor dieser Alternative sind wir auch hinsichtlich der Begriffe der zeitlichen und räumlichen Entfernung gestellt. Die Antwort kann nach meiner Ansicht nur nach Zweckmäßigkeitsgründen gegeben werden; wie sie ausfallen wird, erscheint mir zweifelhaft."

72 "[...] dass man dem symmetrischen Tensor des Gravitationspotentials einen antisymmetrischen Tensor hinzufügte, der den Sechservektor des elektromagnetischen Feldes repräsentierte. Aber eine genauere Überlegung zeigt, dass man so zu keiner vernünftigen Weltfunktion kommt."

73 "Endlich habe ich mich gründlich von der Mieschen Theorie losgemacht und bin zu einer anderen Stellung zum Problem der Materie gelangt. Die Feldphysik erscheint mir keineswegs mehr als der Schlüssel zu der Wirklichkeit; sondern das Feld, der Äther, ist mir nur noch der in sich selbst völlig kraftlose Übermittler der Wirkungen, die Materie aber eine jenseits des Feldes liegende und dessen Zustände verursachende Realität."
} 
to be made. Only success can decide whether such a procedure finds its justification $[\ldots] . " 74[72]$

During the twenties Einstein changed his mind and looked for solutions of his field equations which were everywhere regular to represent matter particles:

"In the program, Mr. Einstein expressed during his two talks given in November 1929 at the Institut Henri Poincaré, he wished to search for the physical laws in solutions of his equations without singularities - with matter and the electromagnetic field thus being continuous. Let us move into the field chosen by him without too much surprise to see him apparently follow a road opposed to the one successfully walked by the contemporary physicists." 75 ([36], p. 17 (1178))

\footnotetext{
74 "Die hier vertretene physikalische Interpretation der Geometrie (Kontinuumstheorie) versagt zwar bei ihrer unmittelbaren Anwendung auf Räume von submolekularer Grössenordnung. Einen Teil ihrer Bedeutung behält sie indessen auch noch den Fragen der Konstitution der Elementarteilchen gegenüber. Denn man kann versuchen, denjenigen Feldbegriffen [...] auch dann physikalische Bedeutung zuzuschreiben, wenn es sich um die Beschreibung der elektrischen Elementarteilchen handelt, die die Materie konstituieren. Nur der Erfolg kann über die Berechtigung eines solchen Verfahrens entscheiden [...]."

75 "Dans le programme qu'a esquissé M. Einstein dans ses deux conférences faites en novembre 1929 à l'institut Henri Poncaré, il voulait chercher les lois physiques dans les solutions sans singularité de ses equations, la matière et l'electricité n'existant donc qu'à l'état continu. Placons-nous sur le terrain choisi par lui, sans trop nous étonner de le voir suivre en apparence une voie opposée à celle suivi avec succès par les physiciens contemporains."
} 


\section{The Main Ideas for Unification between about 1918 and 1923}

After 1915, Einstein first was busy with extracting mathematical and physical consequences from general relativity (Hamiltonian, exact solutions, the energy conservation law, cosmology, gravitational waves). Although he kept thinking about how to find elementary particles in a field theory [70] and looked closer into Weyl's theory [72], at first he only reacted to the new ideas concerning unified field theory as advanced by others. The first such idea after Förster's, of course, was Hermann Weyl's gauge approach to gravitation and electromagnetism, unacceptable to Einstein and to Pauli for physical reasons [246, 292].

Next came Kaluza's five-dimensional unification of gravitation and electromagnetism, and Eddington's affine geometry.

\subsection{Weyl's theory}

\subsubsection{The geometry}

Weyl's fundamental idea for generalising Riemannian geometry was to note that, unlike for the comparison of vectors at different points of the manifold, for the comparison of scalars the existence of a connection is not required. Thus, while lengths of vectors at different points can be compared without a connection, directions cannot. This seemed too special an assumption to Weyl for a genuine infinitesimal geometry:

"If we make no further assumption, the points of a manifold remain totally isolated from each other with regard to metrical structure. A metrical relationship from point to point will only then be infused into [the manifold] if a principle for carrying the unit of length from one point to its infinitesimal neighbours is given." 76

In contrast to this, Riemann made the much stronger assumption that line elements may be compared not only at the same place but also at two arbitrary places at a finite distance.

"However, the possibility of such a comparison 'at a distance' in no way can be admitted in a pure infinitesimal geometry." 77 ([397], p. 397)

In order to invent a purely "infinitesimal" geometry, Weyl introduced the 1-dimensional, Abelian group of gauge transformations,

$$
g \rightarrow \bar{g}:=\lambda g,
$$

besides the diffeomorphism group (coordinate transformations). At a point, Equation (98) induces a local recalibration of lengths $l$ while preserving angles, i.e., $\delta l=\lambda l$. If the non-metricity tensor is assumed to have the special form $Q_{i j k}=Q_{k} g_{i j}$, with an arbitrary vector field $Q_{k}$, then as we know from Equation (57), with regard to these gauge transformations

$$
Q_{k} \rightarrow Q_{k}+\partial_{k} \sigma
$$

We see a striking resemblance with the electromagnetic gauge transformations for the vector potential in Maxwell's theory. If, as Weyl does, the connection is assumed to be symmetric (i.e., with

\footnotetext{
76 "Machen wir keine weitere Voraussetzung, so bleiben die einzelnen Punkte der Mannigfaltigkeit in metrischer Hinsicht vollständig gegeneinander isoliert. Ein metrischer Zusammenhang von Punkt zu Punkt wird erst dann in sie hineingetragen, wenn ein Prinzip der Übertragung der Längeneinheit von einem Punkte P zu seinem unendlich benachbarten vorliegt."

77 "Die Möglichkeit einer solchen 'ferngeometrischen' Vergleichung kann aber in einer reinen Infinitesimalgeometrie durchaus nicht zugestanden werden."
} 
vanishing torsion), then from Equation (42) we get

$$
\Gamma_{i j}{ }^{k}=\left\{{ }_{i j}^{k}\right\}+\frac{1}{2}\left(\delta_{i}^{k} Q_{j}+\delta_{j}^{k} Q_{i}-g_{i j} g^{k l} Q_{l}\right) .
$$

Thus, unlike in Riemannian geometry, the connection is not fully determined by the metric but depends also on the arbitrary vector function $Q_{i}$, which Weyl wrote as a linear form $d Q=Q_{i} d x^{i}$. With regard to the gauge transformations (98), $\Gamma_{i j}{ }^{k}$ remains invariant. From the 1-form $d Q$, by exterior derivation a gauge-invariant 2-form $F=F_{i j} d x^{i} \wedge d x^{j}$ with $F_{i j}=Q_{i, j}-Q_{j, i}$ follows. It is named "Streckenkrümmung" ("line curvature") by Weyl, and, by identifying $Q$ with the electromagnetic 4-potential, he arrived at the electromagnetic field tensor $F$.

Let us now look at what happens to parallel transport of a length, e.g., the norm $|X|$ of a tangent vector along a particular curve $C$ with parameter $u$ to a different (but infinitesimally neighbouring) point:

$$
\frac{d|X|}{d u}=|X|_{\| k} X^{k}=\left(\sigma-\frac{1}{2} Q_{k} X^{k}\right)|X|
$$

By a proper choice of the curve's parameter, we may write (101) in the form $d|X|=-Q_{k} X^{k}|X| d u$ and integrate along $C$ to obtain $|X|=\int \exp \left(-Q_{k} X^{k} d u\right)$. If $X$ is taken to be tangent to $C$, i.e., $X^{k}=\frac{d x^{k}}{d u}$, then

$$
\left|\frac{d x^{k}}{d u}\right|=\int \exp \left(-Q_{k}(x) d x^{k}\right),
$$

i.e., the length of a vector is not integrable; its value generally depends on the curve along which it is parallely transported. The same holds for the angle between two tangent vectors in a point (cf. Equation (44)). For a vanishing electromagnetic field, the 4-potential becomes a gradient ("pure gauge"), such that $Q_{k} d x^{k}=\frac{\partial \omega}{\partial x^{k}} d x^{k}=d \omega$, and the integral becomes independent of the curve.

Thus, in Weyl's connection (100), both the gravitational and the electromagnetic fields, represented by the metrical field $g$ and the vector field $Q$, are intertwined. Perhaps, having in mind Mie's ideas of an electromagnetic world view and Hilbert's approach to unification, in the first edition of his book, Weyl remained reserved:

"Again physics, now the physics of fields, is on the way to reduce the whole of natural phenomena to one single law of nature, a goal to which physics already once seemed close when the mechanics of mass-points based on Newton's Principia did triumph. Yet, also today, the circumstances are such that our trees do not grow into the sky." 78 ([396], p. 170; preface dated "Easter 1918")

However, a little later, in his paper accepted on 8 June 1918, Weyl boldly claimed:

"I am bold enough to believe that the whole of physical phenomena may be derived from one single universal world-law of greatest mathematical simplicity." 79 ([397], p. 385, footnote 4)

The adverse circumstances alluded to in the first quotation might be linked to the difficulties of finding a satisfactory Lagrangian from which the field equations of Weyl's theory can be derived. Due to the additional group of gauge transformations, it is useful to introduce the new concept

\footnotetext{
78 "Wieder ist die Physik, heute die Feldphysik, auf dem Wege, die Gesamtheit der Naturerscheinungen auf ein einziges Naturgesetz zurückzuführen, ein Ziel, dem sie schon einmal, als die durch Newtons Principia begründete mechanische Massenpunkt-Physik ihre Triumphe feierte, nahe zu sein. Doch ist auch heute dafür gesorgt, dass unsere Bäume nicht in den Himmel wachsen."

79 "Ich bin verwegen genug, zu glauben, dass die Gesamtheit der physikalischen Erscheinungen sich aus einem einzigen universellen Weltgesetz von höchster mathematischer Einfachheit herleiten lässt."
} 
of gauge-weight within tensor calculus as in Section 2.1.5 ${ }^{80}$. As the Lagrangian $\mathcal{L}=\sqrt{-g} L$ must have gauge-weight $w=0$, we are looking for a scalar $L$ of gauge-weight -2 . Weitzenböck ${ }^{81}$ has shown that the only possibilities quadratic in the curvature tensor and the line curvature are given by the four expressions [391]

$$
\left(K_{i j} g^{i j}\right)^{2}, \quad K_{i j} K_{k l} g^{i k} g^{j l}, \quad K_{j k l}^{i} K_{i m n}^{j} g^{k m} g^{l n}, \quad F_{i j} F_{k l} g^{i k} g^{j l} .
$$

While the last invariant would lead to Maxwell's equations, from the invariants quadratic in curvature, in general field equations of fourth order result.

Weyl did calculate the curvature tensor formed from his connection (100) but did not get the correct result ${ }^{82}$; it is given by Schouten ([310], p. 142) and follows from Equation (51):

$$
K_{j k l}^{i}=R_{j k l}^{i}+Q_{j ;[k} \delta_{l]}^{i}+\delta_{j}^{i} Q_{[l ; k]}-Q_{;[k}^{i} g_{l] j}+\frac{1}{2}\left(-\delta_{[l}^{i} Q_{k]} Q_{j}+Q_{[k} g_{l] j} Q^{i}-\delta_{[k}^{i} g_{l] j} Q_{r} Q^{r}\right) .
$$

If the metric field $g$ and the 4-potential $Q_{i} \equiv A_{i}$ are varied independently, from each of the curvature-dependent scalar invariants we do get contributions to Maxwell's equations.

Perhaps Bach (alias Förster) was also dissatisfied with Weyl's calculations: He went through the entire mathematics of Weyl's theory, curvature tensor, quadratic Lagrangian field equations and all; he even discussed exact solutions. His Lagrangian is given by $\mathcal{L}=\sqrt{g}\left(3 W_{4}-6 W_{3}+W_{2}\right)$, where the invariants are defined by

$$
W_{4}:=S_{p q i k} S^{p q i k}, \quad W_{3}:=g^{i k} g^{l m} F_{i k q}^{p} F_{l m p}^{q}, \quad W_{2}:=g^{i k} F^{p}{ }_{i k p}
$$

with

$$
\begin{aligned}
S_{[p q][i k]}:= & \frac{1}{4}\left(F_{p q i k}-F_{q p i k}+F_{i k p q}-F_{k i p q}\right), \\
F_{p q i k}= & R_{p q i k}+\frac{1}{2}\left(g_{p q} f_{k i}+g_{p k} f_{q ; i}+g_{q i} f_{p ; k}-g_{p i} f_{q ; k}-g_{q k} f_{p ; i}\right) \\
& +\frac{1}{2}\left(f_{q} g_{p ;[k} f_{i]}+f_{p} g_{q ;[k} f_{i]}+g_{p[i} g_{k] q} f_{r} f^{r}\right),
\end{aligned}
$$

where $R_{p q i k}$ is the Riemannian curvature tensor, $f_{i k}=f_{i, k}-f_{k, i}$, and $f_{i}$ is the electromagnetic 4-potential [4].

\subsubsection{Physics}

While Weyl's unification of electromagnetism and gravitation looked splendid from the mathematical point of view, its physical consequences were dire: In general relativity, the line element $d s$ had been identified with space- and time intervals measurable by real clocks and real measuring rods. Now, only the equivalence class $\left\{\lambda g_{i k} \mid \lambda\right.$ arbitrary $\}$ was supposed to have a physical meaning: It was as if clocks and rulers could be arbitrarily "regauged" in each event, whereas in Einstein's theory the same clocks and rulers had to be used everywhere. Einstein, being the first expert who could keep an eye on Weyl's theory, immediately objected, as we infer from his correspondence with Weyl.

\footnotetext{
${ }^{80}$ In order to distinguish it from the "coordinate-weight" connected with relative tensors [27]. In his book, Weyl just uses the expression "weight".

81 Roland Weitzenböck (1885-1955). Studied mathematics at the University of Vienna where he obtained his doctoral degree in 1910. Became a professional officer during the First World War. He obtained professorships in Graz and Vienna and, in 1921, at the University of Amsterdam. He specialised in the theory of invariants (cf. [156]).

${ }^{82}$ See Section IV, pp. 403-404 in [397]. Weyl's expression violates Equation (38): His curvature tensor allows for a trace $V_{k l} \neq 0$. In fact $V_{k l}=\alpha Q_{[l ; k]}$ is proportional to the electromagnetic field tensor.
} 
In spring 1918, the first edition of Weyl's famous book on differential geometry, special and general relativity Raum-Zeit-Materie appeared, based on his course in Zürich during the summer term of 1917 [396]. Weyl had arranged that the page proofs be sent to Einstein. In communicating this on 1 March 1918, he also stated that

"As I believe, during these days I succeeded in deriving electricity and gravitation from the same source. There is a fully determined action principle, which, in the case of vanishing electricity, leads to your gravitational equations while, without gravity, it coincides with Maxwell's equations in first order. In the most general case, the equations will be of 4 th order, though." 83

He then asked whether Einstein would be willing to communicate a paper on this new unified theory to the Berlin Academy ([321], Volume 8B, Document 472, pp. 663-664). At the end of March, Weyl visited Einstein in Berlin, and finally, on 5 April 1918, he mailed his note to him for the Berlin Academy. Einstein was impressed: In April 1918, he wrote four letters and two postcards to Weyl on his new unified field theory - with a tone varying between praise and criticism. His first response of 6 April 1918 on a postcard was enthusiastic:

"Your note has arrived. It is a stroke of genious of first rank. Nevertheless, up to now I was not able to do away with my objection concerning the scale." ${ }^{4}$ ([321], Volume 8B, Document 498, 710)

Einstein's "objection" is formulated in his "Addendum" ("Nachtrag") to Weyl's paper in the reports of the Academy, because Nernst had insisted on such a postscript. There, Einstein argued that if light rays would be the only available means for the determination of metrical relations near a point, then Weyl's gauge would make sense. However, as long as measurements are made with (infinitesimally small) rigid rulers and clocks, there is no indeterminacy in the metric (as Weyl would have it): Proper time can be measured. As a consequence follows: If in nature length and time would depend on the pre-history of the measuring instrument, then no uniquely defined frequencies of the spectral lines of a chemical element could exist, i.e., the frequencies would depend on the location of the emitter. He concluded with the words

"Regrettably, the basic hypothesis of the theory seems unacceptable to me, [of a theory] the depth and audacity of which must fill every reader with admiration." ${ }^{55}$ ([395], Addendum, p. 478)

Einstein's remark concerning the path-dependence of the frequencies of spectral lines stems from the path-dependency of the integral (102) given above. Only for a vanishing electromagnetic field does this objection not hold.

Weyl answered Einstein's comment to his paper in a "reply of the author" affixed to it. He doubted that it had been shown that a clock, if violently moved around, measures proper time $\int d s$. Only in a static gravitational field, and in the absence of electromagnetic fields, does this hold:

"The most plausible assumption that can be made for a clock resting in a static field is this: that it measure the integral of the $d s$ normed in this way [i.e., as in Einstein's

\footnotetext{
83 "Diese Tage ist es mir, wie ich glaube, gelungen, Elektrizität und Gravitation aus einer gemeinsamen Quelle herzuleiten. Es ergibt sich ein völlig bestimmtes Wirkungsprinzip, das im elektrizitätsfreien Fall auf Ihre Gravitationsgleichungen führt, im gravitationsfreien dagegen Gleichungen ergibt, die in erster Näherung mit den Maxwellschen übereinstimmen. Im allgemeinsten Fall werden die Gleichungen allerdings 4. Ordnung."

84 "Ihre Abhandlung ist gekommen. Es ist ein Genie-Streich ersten Ranges. Allerdings war ich nicht imstande, meinen Massstab-Einwand zu erledigen."

85 “[...] scheint mir die Grundhypothese der Theorie leider nicht annehmbar, deren Tiefe und Kühnheit aber jeden Leser mit Bewunderung erfüllen muss."
} 
theory]; the task remains, in my theory as well as in Einstein's, to derive this fact by a dynamics carried through explicitly." 86 ([395], p. 479)

Einstein saw the problem, then unsolved within his general relativity, that Weyl alluded to, i.e., to give a theory of clocks and rulers within general relativity. Presumably, such a theory would have to include microphysics. In a letter to his former student Walter Dällenbach, he wrote (after 15 June 1918):

"[Weyl] would say that clocks and rulers must appear as solutions; they do not occur in the foundation of the theory. But I find: If the $d s$, as measured by a clock (or a ruler), is something independent of pre-history, construction and the material, then this invariant as such must also play a fundamental role in theory. Yet, if the manner in which nature really behaves would be otherwise, then spectral lines and well-defined chemical elements would not exist. [...] In any case, I am as convinced as Weyl that gravitation and electricity must let themselves be bound together to one and the same; I only believe that the right union has not yet been found." ${ }^{87}$ ([321], Volume 8B, Document 565, 803)

Another famous theoretician who could not side with Weyl was H. A. Lorentz; in a paper on the measurement of lengths and time intervals in general relativity and its generalisations, he contradicted Weyl's statement that the world-lines of light-signals would suffice to determine the gravitational potentials [211].

However, Weyl still believed in the physical value of his theory. As further "extraordinarily strong support for our hypothesis of the essence of electricity" he considered the fact that he had obtained the conservation of electric charge from gauge-invariance in the same way as he had linked with coordinate-invariance earlier, what at the time was considered to be "conservation of energy and momentum", where a non-tensorial object stood in for the energy-momentum density of the gravitational field ([398], pp. 252-253).

Moreover, Weyl had some doubts about the general validity of Einstein's theory which he derived from the discrepancy in value by 20 orders of magniture of the classical electron radius and the gravitational radius corresponding to the electron's mass ([397], p. 476; [152]).

\subsubsection{Reactions to Weyl's theory I: Einstein and Weyl}

There exists an intensive correspondence between Einstein and Weyl, now completely available in volume 8 of the Collected Papers of Einstein [321]. We subsume some of the relevant discussions. Even before Weyl's note was published by the Berlin Academy on 6 June 1918, many exchanges had taken place between him and Einstein.

On a postcard to Weyl on 8 April 1918, Einstein reaffirmed his admiration for Weyl's theory, but remained firm in denying its applicability to nature. Weyl had given an argument for dimension 4 of space-time that Einstein liked: As the Lagrangian for the electromagnetic field $F_{i k} F^{i k}$ is of gauge-weight -2 and $\sqrt{-g}$ has gauge-weight $D / 2$ in an $M_{D}$, the integrand in the Hamiltonian principle $\sqrt{-g} F_{i k} F^{i k}$ can have weight zero only for $D=4$ : "Apart from the [lacking] agreement

\footnotetext{
86 "Die plausibelste Annahme, die man über ein im statischen Feld ruhende Uhr machen kann, ist die, dass sie das Integral des so normierten [d. h. so wie in der Einsteinschen Theorie] $d s$ misst; es bleibt in meiner wie in der Einsteinschen Theorie die Aufgabe ${ }^{1}$, diese Tatsache aus einer explizit durchgeführten Dynamik abzuleiten."

87 “[Weyl] würde sagen, Uhren und Massstäbe müssten erst als Lösungen auftreten; im Fundament der Theorie kommen sie nicht vor. Aber ich finde: Wenn das mit einer Uhr (bzw. einem Massstab) gemessene $d s$ ein von der Vorgeschichte, dem Bau und dem Material Unabhängiges ist, so muss diese Invariante als solche auch in der Theorie eine ganz fundamentale Rolle spielen. Wenn aber die Art des wirklichen Naturgeschehens nicht so wäre, so gäbe es keine Spektrallinien und keine wohldefinierten chemischen Elemente. [...] Jedenfalls bin ich mit Weyl überzeugt, dass Gravitation und Elektrizität zu einem Einheitlichen sich verbinden lassen müssen, nur glaube ich, dass die richtige Verbindung noch nicht gefunden ist."
} 
with reality it is in any case a grandiose intellectual performance" 88 ([321], Vol. 8B, Doc. 499, 711). Weyl did not give in:

"Your rejection of the theory for me is weighty; [... But my own brain still keeps believing in it. And as a mathematician I must by all means hold to [the fact] that my geometry is the true geometry 'in the near', that Riemann happened to come to the special case $F_{i k}=0$ is due only to historical reasons (its origin is the theory of surfaces), not to such that matter." 89 ([321], Volume 8B, Document 544, 767)

After Weyl's next paper on "pure infinitesimal geometry" had been submitted, Einstein put forward further arguments against Weyl's theory. The first was that Weyl's theory preserves the similarity of geometric figures under parallel transport, and that this would not be the most general situation (cf. Equation (49)). Einstein then suggested the affine group as the more general setting for a generalisation of Riemannian geometry ([321], Vol. 8B, Doc. 551, 777). He repeated this argument in a letter to his friend Michele Besso from his vacations at the Baltic Sea on 20 August 1918, in which he summed up his position with regard to Weyl's theory:

"[Weyl's] theoretical attempt does not fit to the fact that two originally congruent rigid bodies remain congruent independent of their respective histories. In particular, it is unimportant which value of the integral $\int \phi_{\nu} d x_{\nu}$ is assigned to their world line. Otherwise, sodium atoms and electrons of all sizes would exist. But if the relative size of rigid bodies does not depend on past history, then a measurable distance between two (neighbouring) world-points exists. Then, Weyl's fundamental hypothesis is incorrect on the molecular level, anyway. As far as I can see, there is not a single physical reason for it being valid for the gravitational field. The gravitational field equations will be of fourth order, against which speaks all experience until now [...]."90 ([99], p. 133)

Einstein's remark concerning "affine geometry" is referring to the affine geometry in the sense it was introduced by Weyl in the 1st and 2nd edition of his book [396], i.e., through the affine group and not as a suggestion of an affine connexion.

From Einstein's viewpoint, in Weyl's theory the line element $d s$ is no longer a measurable quantity - the electromagnetical 4-potential never had been one. Writing from his vacations on 18 September 1918, Weyl presented a new argument in order to circumvent Einstein's objections. The quadratic form $R g_{i k} d x^{i} d x^{k}$ is an absolute invariant, i.e., also with regard to gauge transformations (gauge weight 0 ). If this expression would be taken as the measurable distance in place of $d s$, then

"[...] by the prefixing of this factor, so to speak, the absolute norming of the unit of length is accomplished after all" 91 ([321], Volume 8B, Document 619, 877-879)

88 "Abgesehen von der Übereinstimmung mit der Wirklichkeit ist es jedenfalls eine grandiose Leistung des Gedanken."

89 "Ihre Ablehnung der Theorie fällt für mich schwer ins Gewicht; [...] Aber mein eigenes Hirn bewahrt noch den Glauben an sie. Und daran muss ich als Mathematiker durchaus festhalten: Meine Geometrie ist die wahre Nahegeometrie, dass Riemann nur auf den Spezialfall $F_{i k}=0$ geriet, hat lediglich historische Gründe (Entstehung aus der Flächentheorie), keine sachlichen."

90 “[Weyl's] theoretischer Versuch, passt nicht zu der Thatsache, dass zwei ursprünglich kongruente feste Körper auch kongruent bleiben, unabhängig davon, welche Schicksale sie durchmachen. Insbesondere hat es keine Bedeutung, welcher Wert des Integrals $\int \phi_{\nu} d x_{\nu}$ ihrer Weltlinie zukommt. Sonst würde es Natrium-Atome und Elektronen in allen Grössen geben müssen. Wenn aber die relative Grösse starrer Körper von der Vorgeschichte unabhängig ist, dann gibt es einen messbaren Abstand zweier (benachbarter) Weltpunkte. Dann ist die Weylsche Grundannahme jedenfalls nicht richtig für das molekulare. Dafür, dass sie für das Gravitationsfeld zutreffe, spricht, soweit ich sehe, kein einziger physikalischer Grund. Dagegen aber spricht, dass die Feldgleichungen der Gravitation von vierter Ordnung werden, wofür die bisherige Erfahrung keinerlei Anhalts bietet [...]."

91 "[...] durch Vorsetzen dieses Faktors wird dann doch sozusagen die absolute Normierung der Längeneinheit vollzogen." 
Einstein was unimpressed:

"But the expression $R g_{i k} d x^{i} d x^{k}$ for the measured length is not at all acceptable in my opinion because $R$ is very dependent on the matter density. A very small change of the measuring path would strongly influence the integral of the square root of this quantity." 92

Einstein's argument is not very convincing: $g_{i k}$ itself is influenced by matter through his field equations; it is only that now $R$ is algebraically connected to the matter tensor. In view of the more general quadratic Lagrangian needed in Weyl's theory, the connection between $R$ and the matter tensor again might become less direct. Einstein added:

"Of course I know that the state of the theory as I presented it is not satisfactory, not to speak of the fact that matter remains unexplained. The unconnected juxtaposition of the gravitational terms, the electromagnetic terms, and the $\lambda$-terms undeniably is a result of resignation.[...] In the end, things must arrange themselves such that actiondensities need not be glued together additively." ${ }^{93}$ ([321], Volume 8B, Document 626, 893-894)

The last remarks are interesting for the way in which Einstein imagined a successful unified field theory.

\subsubsection{Reactions to Weyl's theory II: Schouten, Pauli, Eddington, and others}

Sommerfeld seems to have been convinced by Weyl's theory, as his letter to Weyl on 3 June 1918 shows:

"What you say here is really marvelous. In the same way in which Mie glued to his consequential electrodynamics a gravitation which was not organically linked to it, Einstein glued to his consequential gravitation an electrodynamics (i.e., the usual electrodynamics) which had not much to do with it. You establish a real unity."94 [327]

Schouten, in his attempt in 1919 to replace the presentation of the geometrical objects used in general relativity in local coordinates by a "direct analysis", also had noticed Weyl's theory. In his "addendum concerning the newest theory of Weyl", he came as far as to show that Weyl's connection is gauge invariant, and to point to the identification of the electromagnetic 4-potential. Understandably, no comments about the physics are given ([295], pp. 89-91).

In the section on Weyl's theory in his article for the Encyclopedia of Mathematical Sciences, Pauli described the basic elements of the geometry, the loss of the line-element $d s$ as a physical variable, the convincing derivation of the conservation law for the electric charge, and the too many possibilities for a Lagrangian inherent in a homogeneous function of degree 1 of the invariants (103). As compared to his criticism with respect to Eddington's and Einstein's later unified field theories, he is speaking softly, here. Of course, as he noted, no progress had been made with regard to

\footnotetext{
92 "Der Ausdruck $R g_{i k} d x^{i} d x^{k}$ für die gemessene Länge ist aber, wenn man für $R$ die Krümmungsinvariante nimmt, nach meiner Meinung keineswegs akzeptabel, weil $R$ sehr abhängig ist von der materiellen Dichte. Eine ganz kleine Änderung des Messweges würde das Integral der Quadratzwurzel dieser Grösse sehr stark beeinflussen."

93 "Natürlich weiss ich, dass der Zustand der Theorie, wie ich ihn hingestellt habe, ein nicht befriedigender ist, abgesehen davon, dass die Materie unerklärt bleibt. Die zusammenhanglose Nebeneinandersetzung der Gravitationsglieder, der elektromagnetischen Glieder und der $\lambda$-Glieder ist unleugbar ein Produkt der Resignation. [...] Endlich muss es so herauskommen, dass man nicht Wirkungsdichten additiv aneinander kleben muss."

94 "Was Sie da sagen, ist wirklich wundervoll. So wie Mie seiner konsequenten Elektrodynamik eine Gravitation angeklebt hatte, die nicht organisch mit jener zusammenhing, ebenso hat Einstein seiner konsequenten Gravitation eine Elektrodynamik (d.h. die gewöhliche Elektrodynamik) angeklebt, die mit jener nicht viel zu tun hatte. Sie stellen eine wirkliche Einheit her."
}

Living Reviews in Relativity

http: //www . livingreviews . org/lrr-2004-2 
the explanation of the constituents of matter; on the one hand because the differential equations were too complicated to be solved, on the other because the observed mass difference between the elementary particles with positive and negative electrical charge remained unexplained. In his general remarks about this problem at the very end of his article, Pauli points to a link of the asymmetry with time-reflection symmetry (see [246], pp. 774-775; [244]). For Einstein, this criticism was not only directed against Weyl's theory

"but also against every continuum-theory, also one which treats the electron as a singularity. Now as before I believe that one must look for such an overdetermination by differential equations that the solutions no longer have the character of a continuum. But how?" ([103], p. 43)

In a letter to Besso on 26 July 1920, Einstein repeated an argument against Weyl's theory which had been removed by Weyl - if only by a trick to be described below; Einstein thus said:

"One must pass to tensors of fourth order rather than only to those of second order, which carries with it a vast indeterminacy, because, first, there exist many more equations to be taken into account, second, because the solutions contain more arbitrary

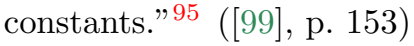

In his book "Space, Time, and Gravitation", Eddington gave a non-technical introduction into Weyl's "welding together of electricity and gravitation into one geometry". The idea of gauging lengths independently at different events was the central theme. He pointed out that while the fourfold freedom in the choice of coordinates had led to the conservation laws for energy and momentum, "in the new geometry is a fifth arbitrariness, namely that of the selected gaugesystem. This must also give rise to an identity; and it is found that the new identity expresses the law of conservation of electric charge." One natural gauge was formed by the "radius of curvature of the world"; "the electron could not know how large it ought to be, unless it had something to measure itself against" ([57], pp. 174, 173, 177).

As Eddington distinguished natural geometry and actual space from world geometry and conceptual space serving for a graphical representation of relationships among physical observables, he presented Weyl's theory in his monograph "The mathematical theory of relativity"

"from the wrong end - as its author might consider; but I trust that my treatment has not unduly obscured the brilliance of what is unquestionably the greatest advance in the relativity theory after Einstein's work." ([59], p. 198)

Of course, "wrong end" meant that Eddington took Weyl's theory such

"that his non-Riemannian geometry is not to be applied to actual space-time; it refers to a graphical representation of that relation-structure which is the basis of all physics, and both electromagnetic and metrical variables appear in it as interrelated." ([59], p. 197)

Again, Eddington liked Weyl's natural gauge encountered in Section 4.1.5, which made the curvature scalar a constant, i.e., $K=4 \lambda$; it became a consequence of Eddington's own natural gauge in his affine theory, $K_{i j}=\lambda g_{i j}$ (cf. Section 4.3). For Eddington, Weyl's theory of gaugetransformation was a hybrid:

"He admits the physical comparison of length by optical methods [...]; but he does not recognise physical comparison of length by material transfer, and consequently he takes

\footnotetext{
95 "Man muss zu Tensoren übergehen die 4. Ordnung sind statt nur zweiter Ordnung, was eine weitgehende Unbestimmtheit der Theorie mit sich bringt, erstens weil es bedeutend mehr Gleichungen gibt, die in Betracht kommen, zweitens, weil die Lösungen mehr willkürliche Konstanten enthalten."
} 
$\lambda$ to be a function fixed by arbitrary convention and not necessarily a constant." ([59], pp. 220-221)

In the depth of his heart Weyl must have kept a fondness for his idea of "gauging" a field all during the decade between 1918 and 1928. As he had abandoned the idea of describing matter as a classical field theory since 1920, the linking of the electromagnetic field via the gauge idea could only be done through the matter variables. As soon as the new spinorial wave function ("matter wave") in Schrödinger's and Dirac's equations emerged, he adapted his idea and linked the electromagnetic field to the gauging of the quantum mechanical wave function [407, 408]. In October 1950, in the preface for the first American printing of the English translation of the fourth edition of his book Space, Time, Matter from 1922, Weyl clearly expressed that he had given up only the particular idea of a link between the electromagnetic field and the local calibration of length:

"While it was not difficult to adapt also Maxwell's equations of the electromagnetic field to this principle [of general relativity], it proved insufficient to reach the goal at which classical field physics is aiming: a unified field theory deriving all forces of nature from one common structure of the world and one uniquely determined law of action.[...] My book describes an attempt to attain this goal by a new principle which I called gauge invariance. (Eichinvarianz). This attempt has failed." ([410], p. V)

\subsubsection{Reactions to Weyl's theory III: Further research}

Pauli, still a student, and with his article for the Encyclopedia in front of him, pragmatically looked into the gravitational effects in the planetary system, which, as a consequence of Einstein's field equations, had helped Einstein to his fame. He showed that Weyl's theory had, for the static case, as a possible solution a constant Ricci scalar; thus it also admitted the Schwarzschild solution and could reproduce all desired effects [244, 243].

Weyl himself continued to develop the dynamics of his theory. In the third edition of his Space-Time-Matter [398], at the Naturforscherversammlung in Bad Nauheim in 1920 [399], and in his paper on "the foundations of the extended relativity theory" in 1921 [402], he returned to his new idea of gauging length by setting $R=\lambda=$ const. (cf. Section 4.1.3); he interpreted $\lambda$ to be the "radius of curvature" of the world. In 1919, Weyl's Lagrangian originally was $\mathcal{L}=$ $\frac{1}{2} \sqrt{g} K^{2}+\beta F_{i k} F^{i k}$ together with the constraint $K=2 \lambda$, with constant $\lambda$ ([398], p. 253). As an equivalent Lagrangian Weyl gave, up to a divergence ${ }^{96}$

$$
\mathcal{L}=\sqrt{g}\left(R+\alpha F_{i k} F^{i k}+\frac{1}{4}\left(2 \lambda-3 \phi_{l} \phi^{l}\right)\right),
$$

with the 4-potential $\phi_{l}$ and the electromagnetic field $F_{i k}$. Due to his constraint, Weyl had navigated around another problem, i.e., the formulation of the Cauchy initial value problem for field equations of fourth order: Now he had arrived at second order field equations. In the paper in 1921, he changed his Lagrangian slightly into

$$
\mathcal{L}=\sqrt{g}\left(R+\alpha F_{i k} F^{i k}+\frac{\epsilon^{2}}{4}\left(1-3 \phi_{l} \phi^{l}\right)\right)
$$

with $\epsilon$ a factor in Weyl's connection (100),

$$
\Gamma_{i j}^{k}=\left\{\begin{array}{l}
k \\
i j
\end{array}\right\}+\frac{\epsilon}{2}\left(\delta_{i}^{k} \phi_{j}+\delta_{j}^{k} \phi_{i}-g_{i j} g^{k l} \phi_{l}\right) .
$$

In both presentations, he considered as an advantage of his theory:

${ }^{96}$ In fact, from Equation (104) we obtain $K=R+3 Q_{l} Q^{l}+3 Q_{; j}^{j}$. 
"Moreover, this theory leads to the cosmological term in a uniform and forceful manner,

[a term] which in Einstein's theory was introduced ad hoc" 97 ([402], p. 474)

Reichenbächer seemingly was unhappy about Weyl's taking the curvature scalar to be a constant before the variation; in the discussion after Weyl's talk in 1920, he inquired whether one could not introduce Weyl's "natural gauge" after the variation of the Lagrangian such that the field equations would show their gauge invariance first ([399], p. 651). Eddington criticised Weyl's choice of a Lagrangian as speculative:

"At the most we can only regard the assumed form of action [...] as a step towards some more natural combination of electromagnetic and gravitational variables." ([59], p. 212)

The changes, which Weyl had introduced in the 4th edition of his book [401], and which, according to him, were of fundamental importance for the understanding of relativity theory, were discussed by him in a further paper [400]. In connection with the question of whether, in general relativity, a formulation might be possible such that "matter whose characteristical traits are charge, mass, and motion generates the field", a question which was considered as unanswered by Weyl, he also mentioned a publication of Reichenbächer [272]. For Weyl, knowledge of the charge and mass of each particle, and of the extension of their "world-channels" were insufficient to determine the field uniquely. Weyl's hint at a solution remains dark; nevertheless, for him it meant

“to reconciliate Reichenbächer's idea: matter causes a 'deformation' of the metrical field and Einstein's idea: inertia and gravitation are one." ([400], p. 561, footnote)

Although Einstein could not accept Weyl's theory as a physical theory, he cherished "its courageous mathematical construction" and thought intensively about its conceptual foundation: This becomes clear from his paper "On a complement at hand of the bases of general relativity" of 1921 [73]. In it, he raised the question whether it would be possible to generate a geometry just from the conformal invariance of Equation (9) without use of the conception "distance", i.e., without using rulers and clocks. He then embarked on conformal invariants and tensors of gauge-weight 0 , and gave the one formed from the square of Weyl's conformal curvature tensor (59), i.e. $C_{j k l}^{i} C_{i}^{j k l}$. His colleague in Vienna, Wirtinger ${ }^{98}$, had helped him in this ${ }^{99}$. Einstein's conclusion was that, by writing down a metric with gauge-weight 0, it was possible to form a theory depending only on the quotient of the metrical components. If $J$ has gauge-weight -1 , then $J g_{i k}$ is such a metric. In order to reduce the new theory to general relativity, in addition only the differential equation

$$
J=J_{0}=\text { const. }
$$

would have to be solved.

Eisenhart wished to partially reinterpret Weyl's theory: In place of putting the vector potential equal to Weyl's gauge vector, he suggested to identify it with $\frac{-F_{k}^{i} J^{k}}{\mu}$, where $J^{i}$ is the electrical 4-current vector (-density) and $\mu$ the mass density. He referred to Weyl, Eddington's book, and to Pauli's article in the Encyclopedia of Mathematical Sciences [116].

\footnotetext{
97 "Ausserdem führt dies Theorie auf einheitliche Weise und zwingend zu dem kosmologischen Gliede, das bei Einstein nur eine ad hoc gemachte Annahme war [...]."

98 Wilhelm Wirtinger (1865-1945). Born in Ybbs, Austria. Studied mathematics at the University of Vienna. Received his doctorate in 1887, and continued his studies at the Universities of Berlin and of Göttingen. From 1895 a full professor at the University of Vienna, but accepted professorship at University of Innsbruck, returning to Vienna only in 1905. Wrote an important paper on the general theta function and had an exceptional range in mathematics (function theory, algebra, number theory, plane geometry, theory of invariants).

${ }^{99}$ In the same year, Wirtinger sent in a paper on relativity theory published only in 1922 [421].
} 
Einstein's rejection of the physical value of Weyl's theory was seconded by Dienes ${ }^{100}$, if only with a not very helpful argument. He demanded that the connection remain metric-compatible from which, trivially, Weyl's gauge-vector must vanish. Dienes applied the same argument to Eddington's generalisation of Weyl's theory [51]. Other mathematicians took Weyl's theory at its face value and drew consequences; thus M. Juvet calculated Frenet's formulas for an " $n$-èdre" in Weyl's geometry by generalising a result of Blaschke for Riemannian geometry [180]. More important, however, for later work was the gauge invariant tensor calculus by a fellow of St. John's College in Cambridge, M. H. A. Newman [237]. In this calculus, tensor equations preserve their form both under a change of coordinates and a change of gauge. Newman applied his scheme to a variational principle with Lagrangian $K^{2}$ and concluded:

"The part independent of the 'electrical' vector $\phi_{i}$ is found to be $K_{i j}-\frac{1}{4} K g_{i j}$, a tensor which has been considered by Einstein from time to time in connection with the theory of gravitation." ([237], p. 623)

After the Second World War, research following Weyl's classical geometrical approach with his original 1-dimensional Abelian gauge-group was resumed. The more important development, however, was the extension to non-Abelian gauge-groups and the combination with Kaluza's idea. We shall discuss these topics in Part II of this article. The shift in Weyl's interpretation of the role of the gauging from the link between gravitation and electromagnetism to a link between the quantum mechanical state function and electromagnetism is touched on in Section 7.

\subsection{Kaluza's five-dimensional unification}

What is now called Kaluza-Klein theory in the physics community is a mixture of quite different contributions by both scientists ${ }^{101}$. Kaluza's idea of looking at four spatial and one time dimension originated in or before 1919; by then he had communicated it to Einstein:

"The idea of achieving [a unified field theory] by means of a five-dimensional cylinder world never dawned on me. [...] At first glance I like your idea enormously." (letter of Einstein to Kaluza of 21 April 1919)

This remark is surprising because Nordström had suggested a five-dimensional unification of his scalar gravitational theory with electromagnetism five years earlier [238], by embedding space-time into a five-dimensional world in quite the same way as Kaluza did. In principle, Einstein could have known Nordström's work. In the same year 1914, he and Fokker had given a covariant formulation of Nordström's pure (scalar) theory of gravitation [104]. In a subsequent letter to Kaluza of 5 May 1919 Einstein still was impressed: "The formal unity of your theory is startling." However, on 29 May 1919, Einstein became somewhat reserved ${ }^{102}$ :

"I respect greatly the beauty and boldness of your idea. But you understand that, in view of the existing factual concerns, I cannot take sides as planned originally." 103

Kaluza's paper was communicated by Einstein to the Academy, but for reasons unknown was published only in 1921 [181]. Kaluza's idea was to write down the Einstein field equations for empty

\footnotetext{
100 Paul Dienes (1982-1952). Born in Tokaj, Hungary. Studied mathematics. From 1929-1945 Reader, and from 1945-48 Professor at Birkbeck College, University of London.

${ }^{101} \mathrm{~A}$ detailed investigation of this subject will appear soon, and will be included in the next update of this article [145].

${ }^{102}$ Some letters of Einstein to Kaluza are quoted by Pais ([241], p. 330), some are reprinted as facsimile in the Proceedings of the Erice Summer School of Cosmology and Gravitation 1982 [49], p. 452.

103 "Ich habe grossen Respekt vor der Schönheit und Kühnheit Ihrer Gedanken. Aber Sie begreifen ja, dass ich bei den obwaltenden sachlichen Bedenken nicht in der ursprünglich geplanten Weise dafür Partei nehmen kann."
}

Living Reviews in Relativity

http://www. livingreviews.org//rr-2004-2 
space in a five-dimensional Riemannian manifold with metric $g_{\alpha \beta}$, i.e., $R_{\alpha \beta}=0, \alpha, \beta=1, \ldots, 5$, where $R_{\alpha \beta}$ is the Ricci tensor of $M_{5}$, and to look at small deviations $\gamma$ from Minkowski space: $g_{\alpha \beta}=-\delta_{\alpha \beta}+\gamma_{\alpha \beta} \cdot{ }^{104}$. In order to obtain a theory in space-time, he assumed the so-called "cylinder condition"

$$
g_{\alpha \beta, 5}=0,
$$

equivalent to the existence of a spacelike translational symmetry (Killing vector). Equation (109) is used for all "functions of state" (Zustandsgrössen), i.e., also for the matter variables. Kaluza did not normalize the Killing vector to a constant, i.e., he kept

$$
g_{55} \neq \text { const. }
$$

Equation (110) is called the "sharpened cylinder condition" by some authors including Einstein. Of the 15 components of $g_{\alpha \beta}$, five had to get a new physical interpretation, i.e. $g_{\alpha 5}$ and $g_{55}$; the components $g_{i k}, i, k=1, \ldots, 4$, were to describe the gravitational field as before; Kaluza took $g_{i 5}$ proportional to the electromagnetic vector potential $A_{i}$. The component $g_{55}$ turned out to be a (scalar) gravitational potential which, in the static case, satisfies the equation

$$
\nabla^{2} g_{55}=-\kappa \mu_{0}
$$

with the constant matter density $\mu_{0}$.

Kaluza also showed that the geodesics of the five-dimensional space reduce to the equations of motion for a charged point particle in space-time, if a weakness assumption is made for the components of the 5 -velocity $u^{\alpha}: u^{1}, u^{2}, u^{3}, u^{5} \ll 1, u^{4} \simeq 1$. The Lorentz force appears augmented by an additional term containing $g_{55}$ of the order $\left(\frac{u}{c}\right)^{2}$ which thus may be neglected. From the fifth equation of motion Kaluza concluded that the fifth component of momentum $p_{5} \sim e$, with $e$ being the particles' electric charge (up to a constant of proportionality). From the equations of motion, charge conservation also followed in Kaluza's linear approximation. Kaluza was well aware that his theory broke down if applied to elementary particles like electrons or protons, and speculated about an escape in which gravitation had to be considered as some "difference effect", and the gravitational constant given "a statistical meaning". For him, any theory claiming universal validity was endangered by quantum theory, anyway.

From the cylinder condition, a grave objection toward Kaluza's approach results: Covariance with regard to the diffeomorphism group of $M_{5}$ is destroyed. The remaining covariance group $G_{5}$ is given by

$$
x^{5^{\prime}}=x^{5}+f\left(x^{k}\right), \quad x^{l^{\prime}}=x^{l}\left(x^{m}\right), \quad k, l, m=1, \ldots, 4 .
$$

The objects transforming properly under (112) are: the scalar $g_{5^{\prime} 5^{\prime}}=g_{55}$, the vector-potential $g_{5^{\prime} k^{\prime}}=g_{5 l} \frac{\partial x^{l}}{\partial x^{k^{\prime}}}+g_{55} \frac{\partial x^{5}}{\partial x^{k^{\prime}}}$, and the projected metric

$$
g_{i^{\prime} k^{\prime}}-\frac{g_{i^{\prime} 5^{\prime}} g_{k^{\prime} 5^{\prime}}}{g_{5^{\prime} 5^{\prime}}}=\left(g_{l m}-\frac{g_{l 5} g_{m 5}}{g_{55}}\right) \frac{\partial x^{l}}{\partial x^{i^{\prime}}} \frac{\partial x^{m}}{\partial x^{k^{\prime}}} .
$$

Klein identified the group; however, he did not comment on the fact that now further invariants are available for a Lagrangian, but started right away from the Ricci scalar of $M_{5}$ [185]. The group $G_{5}$ is isomorphic to the group $H_{5}$ of transformations for five homogeneous coordinates $X^{\mu^{\prime}}=f^{\mu}\left(X^{\nu}\right)$ with $f^{\nu}$ homogeneous functions of degree 1 . Here, contact is made to the projective formulation of Kaluza's theory (cf. "projective geometry" in Sections 2.1.3 and 6.3.2).

While towards the end of May 1919 Einstein had not yet fully supported the publication of Kaluza's manuscript, on 14 October 1921 he thought differently:

\footnotetext{
${ }^{104}$ Greek lettered indices run in $M_{5}$, Latin ones in space time; $x^{4}$ is the time coordinate, $x^{5}$ the new spacelike coordinate.
} 
"I am having second thoughts about having kept you from the publication of your idea on the unification of gravitation and electricity two years ago. I value your approach more than the one followed by H. Weyl. If you wish, I will present your paper to the Academy after all." ${ }^{105}$ (letter from Einstein to Kaluza reprinted in [49], p. 454)

It seems that at some point Einstein had set his calculational aide Grommer ${ }^{106}$ to work on regular spherically symmetric solutions of Kaluza's theory. This led to a joint publication which was submitted just one month after Einstein had finally presented a rewritten manuscript of Kaluza's to the Berlin Academy [105]. The negative result of his own paper, i.e., that no non-singular, statical, spherically symmetric exact solution exists, did not please Einstein. He also thought that Kaluza's assumption of general covariance in the five-dimensional manifold had no support from physics; he disliked the preference of the fifth coordinate due to Equation (109) which seemed to contradict the equivalence of all five coordinates used by Kaluza in the construction of the field equations [105]. In any case, apart from an encouraging letter to Kaluza in 1925 in which he called Kaluza's idea the only serious attempt at unified field theory besides the Weyl-Eddington approach, Einstein kept silent on the five-dimensional theory until 1926.

\subsection{Eddington's affine theory}

\subsubsection{Eddington's paper}

The third main idea that emerged was Eddington's suggestion to forego the metric as a fundamental concept and start right away with a (general) connection, which he then restricted to a symmetric one $\Gamma$ in order to avoid an "infinitely crinkled" world [58]. His motivation went beyond the unification of gravitation and electromagnetism:

"In passing beyond Euclidean geometry, gravitation makes its appearance; in passing beyond Riemannian geometry, electromagnetic force appears; what remains to be gained by further generalisation? Clearly, the non-Maxwellian binding forces which hold together an electron. But the problem of the electron must be difficult, and I cannot say whether the present generalisation succeeds in providing the material for its solution" ([58], p. 104)

In the first, shorter, part of two, Eddington describes affine geometry; in the second he relates mathematical objects to physical variables. He distinguishes the affine geometry as the "geometry of the world-structure" from Riemannian geometry as "the natural geometry of the world". He starts by calculating both the curvature and Ricci tensors from the symmetric connection according to Equation (39). The Ricci tensor $K^{i j}(\Gamma):={ }^{*} G^{i j}$ is asymmetric ${ }^{107}$,

$$
{ }^{*} G_{k l}=R_{k l}+F_{k l},
$$

with $R_{k l}(\Gamma)$ being the symmetric and $F_{k l}(\Gamma)$ the antisymmetric part. According to Equation (31) $F_{k l}$ derives from a "vector potential", i.e., $F_{k l}=\partial_{k} \Gamma_{l}-\partial_{l} \Gamma_{k}$ with $\Gamma_{l}:=\Gamma_{l r}{ }^{r}$, such that an immediate physical identification of $F_{k l}$ with the electromagnetic field tensor is at hand. With half of Maxwell's equations being satisfied automatically, the other half is used to define the electric charge current $j^{k}$ by $j^{l}:=F_{\| k}^{l k}$. By this, Eddington claims to guarantee charge conservation:

\footnotetext{
105 "Ich mache mir Gedanken darüber, dass ich Sie vor zwei Jahren von der Publikation Ihrer Idee über die Vereinigung von Gravitation und Elektrizität abgehalten habe. Ihr Weg scheint mir jedenfalls mehr für sich zu haben als der von H. Weyl beschrittene. Wenn Sie wollen, lege ich Ihre Arbeit doch der Akademie vor [...]."

106 Jakob Grommer (1879-1933). Born near Brest, then in Russia. First a Talmud student with a keen interest in mathematics. Came to Göttingen to study mathematics and obtained his Ph.D. there. Worked with Einstein for at least a decade (1917-1927) as his calculational assistant. He held a university position in Minsk from 1929 on and later became a member of the Belorussian Academy of Sciences. From his youth he was inflicted with elephantiasis.

${ }^{107}$ We use some of Eddington's notation. His notation representing the covariant derivative by a lower index is highly ambiguous, though, and will be avoided. ${ }^{*} G_{k l}$ is not a dual.
} 
"The divergence of $j^{k}$ will vanish identically if $j^{k}$ is itself the divergence of any antisymmetrical contravariant tensor." ([64], p. 223; cf. also [58], p. 113)

Now, by Equation (25),

$$
F_{\|[l \| k]}^{l k}=K_{[r k]} F^{r k}+S_{j k}^{s} \nabla_{s} F^{j k} .
$$

For a symmetric connection thus, unlike in Riemannian geometry,

$$
j_{\| k}^{k}=F_{\|[l \| k]}^{l k}=F_{r k} F^{r k} \neq 0 .
$$

However, for a tensor density, due to Equation (16) we obtain

$$
\hat{\jmath}_{\| k}^{k}=\hat{F}_{\|[l \| k]}^{l k}=\frac{1}{2}\left(V_{r k}+2 K_{[r k]}\right) \hat{F}^{r k}+S_{j k}^{s} \nabla_{s} \hat{F}^{j k}
$$

and thus for a torsionless connection (cf. Equation (38)) $j_{\| k}^{k}=0$.

Eddington introduces the metrical tensor by the definition

$$
\lambda g_{k l}=R_{k l}
$$

"introducing a universal constant $\lambda$, for convenience, in order to remain free to use the centimetre instead of the natural unit of length". This is called "Einstein's gauge" by Eddington; he is delighted that

"Our gauging-equation is therefore certainly true wherever light is propagated, i.e., everywhere inside the electron. Who shall say what is the ordinary gauge inside the electron?" ([58], p. 114)

While this remark certainly is true, there is no guarantee in Eddington's approach that $g_{k l}$ thus defined is a Lorentzian metric, i.e., that it could describe light propagation at all. Only connections leading to a Lorentz metric can be used if a physical interpretation is wanted. Note also, that the interpretation of $R_{k l}$ as the metric implies that det $R_{k l} \neq 0$.

We must read Equation (118) as giving $g_{k l}(\Gamma)$ if the only basic variable in affine geometry, i.e., the connection $\Gamma_{i j}{ }^{k}$, has been determined by help of some field equations. Thus, in general, $g_{k l}$ is not metric-compatible; in order to make it such, we are led to the differential equations $R_{i j \| k}=0$ for $\Gamma_{i j}{ }^{k}$, an equation not considered by Eddington. In the absence of an electromagnetic field, Equation (118) looks like Einstein's vacuum field equation with cosmological constant. In principle, now a fictitious "Riemannian" connection (the Christoffel symbol) can be written down which, however, is a horribly complicated function of the affine connection - as the only fundamental geometrical quantity available. This is due to the expression for the inverse of the metric, a function cubic in $R_{k l}$. Eddington's affine theory thus can also be seen as a bi-connection theory. Note also that Eddington does not explicitly say how to obtain the contravariant form of the electromagnetic field $F^{i j}$ from $F_{i j}$; we must assume that he thought of raising indices with the complicated inverse metric tensor.

In connection with cosmological considerations, Eddington cherished the $\lambda$-term in Equation (118):

"I would as soon think of reverting to Newtonian theory as of dropping the cosmic constant." ([63], p. 35)

Now, Eddington was able to identify the energy-momentum tensor $T^{i k}$ of the electromagnetic field by decomposing the Ricci tensor $K^{i j}$ formed from Equation (51) into a metric part $R_{i k}$ and the rest. The energy-momentum tensor $T^{i k}$ of the electromagnetic field is then defined by Einstein's field equations with a fictitious cosmological constant $\kappa T^{i k}:=G^{i k}-\frac{1}{2} g^{i k}(G-2 \lambda)$. 
Although Eddington's interest did not rest on finding a proper set of field equations, he nevertheless discussed the Lagrangian $\mathcal{L}=\sqrt{-g}{ }^{*} G^{i k}{ }^{*} G_{i k}$, and showed that a variation with regard to $g_{i k}$ did not lead to an acceptable field equation.

Eddington's main goal in this paper was to include matter as an inherent geometrical structure:

"What we have sought is not the geometry of actual space and time, but the geometry of the world-structure which is the common basis of space and time and things." ([58], p. 121)

By "things" he meant

(1) the energy-momentum tensor of matter, i.e., of the electromagnetic field,

(2) the tensor of the electromagnetic field, and

(3) the electric charge-and-current vector.

His aim was reached in the sense that all three quantities were fixed entirely by the connection; they could no longer be given from the outside. As to the question of the electron, it is seen as "a region of abnormal world-curvature", i.e., of abnormally large curvature.

While Pauli liked Eddington's distinction between "natural geometry" and "world geometry" - with the latter being only "a graphical representation" of reality - he was not sure at all whether "a point of view could be taken from which the gravitational and electromagnetical fields appear as union". If so, then it must be a purely phenomenological one without any recourse to the nature of the charged elementary particles (cf. his letter to Eddington quoted below).

Lorentz did not like the large number of variables in Eddington's theory; there were 4 components of the electromagnetic potential, 10 components of the metric and 40 components of the connection:

"It may well be asked whether after all it would not be preferable simply to introduce the functions that are necessary for characterising the electromagnetic and gravitational fields, without encumbering the theory with so great a number of superfluous quantities." ([211], p. 382)

\subsubsection{Einstein's reaction and publications}

Eddington's publication early in 1921, generalising Einstein's and Weyl's theories started a new direction of research both in physics and mathematics. At first, Einstein seems to have been reserved (cf. his letters to Weyl in June and September 1921 quoted by Stachel in his article on Eddington and Einstein ([330], pp. 453-475; here p. 466)), but one and a half years later he became attracted by Eddington's idea. To Bohr, Einstein wrote from Singapore on 11 January 1923:

"I believe I have finally understood the connection between electricity and gravitation.

Eddington has come closer to the truth than Weyl." ([139], p. 274)

He now tried to make Eddington's theory work as a physical theory; Eddington had not given field equations:

"I must absolutely publish since Eddington's idea must be thought through to the end."

(letter of Einstein to Weyl of 23 May 1923; cf. [241], p. 343)

And a few days later, he was still intrigued about this sort of unified field theory, in particular about its elusiveness:

Living Reviews in Relativity

http: //www. livingreviews.org/lrr-2004-2 
"[...] Over it lingers the marble smile of inexorable nature, which has bestowed on us more longing than brains." 108 (letter of Einstein to Weyl of 26 May 1923; cf.[241], p. 343$)$

And indeed Einstein published fast, even while still on the steamer returning from Japan through Palestine and Spain: The paper of February 1923 in the reports of the Berlin Academy carries, as location of the sender, the ship "Haruna Maru" of the Japanese Nippon Yushen Kaisha line ${ }^{109}$ [77].

"In past years, the wish to understand the gravitational and electromagnetic field as one in essence has dominated the endeavours of theoreticians. [...] From a purely logical point of view only the connection should be used as a fundamental quantity, and the metric as a quantity derived thereof [...] Eddington has done this." ${ }^{110}$ ([77], p. 32)

Like Eddington, Einstein used a symmetric connection and wrote down the equation ${ }^{111}$

$$
\lambda^{2} K_{k l}=g_{k l}+\phi_{k l}
$$

where $g_{k l}=g_{(k l)}$ and $\phi_{k l}=\phi_{[k l]}$, and $\lambda$ is a "large number". By this, the metric was defined as the symmetric part of the Ricci tensor. Due to

$$
\phi_{k l}=\frac{1}{2}\left(\frac{\partial \Gamma_{k j}^{j}}{\partial x^{l}}-\frac{\partial \Gamma_{l j}^{j}}{\partial x^{k}}\right)
$$

one half of Maxwell's equations is satisfied if $\phi_{k l}$ is taken to be the electromagnetic field tensor. Let us note, however, that while $\Gamma_{k j}{ }^{j}$ transforms inhomogeneously, its transformation law

$$
\Gamma_{k^{\prime} j^{\prime}}^{j^{\prime}}=\Gamma_{l m}^{m} \frac{\partial x^{l}}{\partial x^{k^{\prime}}}+\frac{\partial^{2} x^{l^{\prime}}}{\partial x^{k^{\prime}} \partial x^{m^{\prime}}} \cdot \frac{\partial x^{m^{\prime}}}{\partial x^{l^{\prime}}}
$$

is not exactly the same as that of the electric 4-potential under gauge transformations.

For a Lagrangian, Einstein used $\mathcal{L}=2 \sqrt{-\operatorname{det} K_{i j}}$; he claims that for vanishing electromagnetic field the vacuum field equations of general relativity, with the cosmological term included, hold. Einstein varied with regard to $g_{k l}$ and $\phi_{k l}$, not, as one might have expected, with regard to the connection $\Gamma_{k j}{ }^{j}$. If $\hat{f}^{k l}:=\frac{\delta \mathcal{L}}{\delta \phi_{k l}}$, then the electric current density $j^{l}$ is defined by $\hat{j}^{l}:=\frac{\partial \hat{f}^{l k}}{\partial x^{k}}$. $\hat{f}^{k l}$ is interpreted as "the contravariant tensor of the electromagnetic field".

The field equations are obtained from the Lagrangian by variation with regard to the connection $\Gamma_{k j}^{l}$ and are (Einstein worked in space-time)

$$
\hat{s}_{\| m}^{k l}+\frac{1}{3} \delta_{m^{j}}^{k}+\frac{1}{3} \delta_{m}^{l} \hat{\jmath}^{k}=0, \quad 3 \hat{s}^{k l}{ }_{\| l}+5 \hat{\jmath}^{k}=0
$$

with the definition of the current density $\hat{j}^{k}$ given before, and $\hat{s}^{k l}=\frac{\delta \mathcal{L}}{\delta g_{k l}}$. Besides $\hat{s}^{k l}$, Einstein also uses $s^{k l}$ introduced by

$$
\hat{s}^{k l}=s^{k l} \sqrt{-\operatorname{det} s_{k l}}, \quad s^{k l} s_{m l}=\delta_{m}^{k} .
$$

\footnotetext{
108 “[...] Darüber steht das marmorne Lächeln der unerbittlichen Natur, die uns mehr Sehnsucht als Geist verliehen hat."

${ }^{109}$ There even exist a manuscript dating from Einstein's stop-over in Singapore; it is incomplete and of little importance [223].

110 "Der Wunsch, das Gravitationsfeld und das elektromagnetische Feld als Wesenseinheit zu begreifen, beherrscht in den letzten Jahren das Streben der Theoretiker. [...] Von einem logisch einleuchtenden Standpunkt her sollte nur die Konnektion als fundamentale Grösse benutzt werden und die Metrik eine daraus abgeleitete Grösse sein. [...] Dies that Eddington."

${ }^{111}$ In place of our $K_{i k}$ Einstein used $R_{i k}$.
} 
From Equation (121) the connection can be obtained. If $\hat{\jmath}^{l}=\sqrt{-\operatorname{det} s_{k l}} j^{l}$, and $j_{k}=s_{k l} j^{l}$, then the affine connection may formally be expressed by

$$
\Gamma_{k j}^{l}=\frac{1}{2} s^{l r}\left(\frac{\partial s_{k r}}{\partial x^{j}}+\frac{\partial s_{j r}}{\partial x^{k}}-\frac{\partial s_{k j}}{\partial x^{r}}\right)-\frac{1}{2} s_{k j \hat{\jmath}^{l}}+\frac{1}{3} \delta_{\left(k \hat{\jmath}_{j}\right)}^{l}
$$

This equation is an identity if a solution of the field equations (121) is inserted. From Equation (123),

$$
\Gamma_{k j}^{j}=\frac{\partial}{\partial x^{k}} \sqrt{\operatorname{det} s_{l r}}+\frac{1}{3} j_{k} .
$$

If no electromagnetic field is present, $\hat{s}^{k l}$ reduces to $\hat{s}^{k l}=g^{k l} \sqrt{-\operatorname{det} g_{k l}}$; the definition of the metric $g_{i j}$ in Equation (119) is reinterpreted by Einstein as giving his vacuum field equation with cosmological constant $\lambda^{-2}$. In order that this makes sense, the identifications in Equation (119) are always to be made after the variation of the Lagrangian is performed.

For non-vanishing electromagnetic field, due to Equation (124) the Equation (120) now becomes

$$
\hat{\phi}_{k l}=\frac{1}{6}\left(\frac{\partial \hat{\jmath}_{k}}{\partial x^{l}}-\frac{\partial \hat{\jmath}_{l}}{\partial x^{k}}\right),
$$

which means that for vanishing current density no electromagnetic field is possible. Einstein concluded:

"But the extraordinary smallness of $\frac{1}{\lambda^{2}}$ implies that finite $\phi_{k l}$ are possible only for tiny, almost vanishing current density. Except for singular positions, the current density is practically vanishing." 112

Einstein went on to show that Maxwell's vacuum equations are holding in first order approximation. $\mathrm{Up}$ to the same order, $\hat{f}^{k l} \simeq \hat{\phi}_{k l}$. In general however, $\hat{\phi}_{k l} \neq s_{k m} s_{l n} \hat{f}^{n m}$. Also, the geometrical theory presented here is energetically closed, i.e., the current density $\hat{\jmath}$ cannot be given arbitrarily as in the usual Maxwell theory with external sources.

Einstein was not sure whether "electrical elementary elements", i.e., nonsingular electrons, are possible in this theory; they might be. He found it remarkable "[...] that, according to this theory, positive and negative electricity cannot differ just in sign" ${ }^{113}$ ([77], p. 38). His final conclusion was:

"that EDDINGTON'S general idea in context with the Hamiltonian principle leads to a theory almost free of ambiguities; it does justice to our present knowledge about gravitation and electricity and unifies both kinds of fields in a truly accomplished manner." 114 ([77], p. 38)

Until the end of May 1923, two further publications followed in which Einstein elaborated on the theory. In the second paper, he exchanged the Lagrangian $\mathcal{L}=2 \sqrt{-\operatorname{det} K_{i j}}$ for a new one, i.e., for $\mathcal{L}=-2 \sqrt{\operatorname{det} K_{i j}}+\hat{R}-\frac{1}{6} \hat{s}^{l m} i_{l} i_{m}$, where $\hat{\imath}^{k}=i^{k} \sqrt{\operatorname{det} s_{k l}}$. $\mathcal{L}$ is to be varied with respect

\footnotetext{
112 "Aber die ausserordentliche Kleinheit von $\frac{1}{\lambda^{2}}$ bringt es mit sich, dass endliche $\phi_{k l}$ nur bei winzigen, praktisch verschwindenden kovarianten Stromdichten möglich sind. Singuläre Stellen ausgenommen verschwindet praktisch also die Stromdichte."

113 “[...] dass nach dieser Theorie die positive und die negative Elektrizität keineswegs bloss dem Vorzeichen nach verschieden sein können."

114 "dass EDDINGTONS allgemeiner Gedanke in Verbindung mit dem Hamiltonschen Prinzip zu einer von Willkür fast freien Theorie führt, welche unserem bisherigen Wissen über Gravitation und Elektrizität gerecht wird und beide Feldarten in wahrhaft vollendeter Weise vereinigt."
} 
to $\hat{s}^{k l}$ and $\hat{f}^{k l}$. The resulting equations for the gravitational and electromagnetic fields are the symmetric and skew-symmetric part, respectively, of

$$
K_{j k}=R_{j k}+\frac{1}{6}\left(\frac{\partial i_{j}}{\partial x^{k}}-\frac{\partial i_{k}}{\partial x^{j}}+i_{j} i_{k}\right) .
$$

Although the theory offered, for every solution with positive charge, also a solution with negative charge, the masses in the two cases were the same. However, the only known particle with positive charge at the time (what is now called the proton) had a mass greatly different from the particle with negative charge, the electron. Einstein noted:

"Therefore, the theory may not account for the difference in mass of positive and negative electrons." ${ }^{115}$ ([74], p. 77)

In the third paper [76], apart from changing notations ${ }^{116}$, Einstein set $\lambda=1$. He also dropped the assumption (119) and replaced it by allowing his Lagrangian (Hamiltonian) $\hat{H}$ to be a function of the two independent variables,

$$
\gamma_{i j}=K_{(i j)}, \quad \phi_{i j}=K_{[i j]} .
$$

The logic of the subsequent derivations in his paper is quite involved. The first step consisted in the definition of tensor densities

$$
\hat{g}^{k l}:=\frac{\delta \hat{H}}{\delta \gamma_{k l}}, \quad \hat{f}^{k l}:=\frac{\delta \hat{H}}{\delta \phi_{k l}} .
$$

In the second step, the variations $\delta \gamma_{k l}$ and $\delta \phi_{k l}$ were expressed by $\delta \Gamma_{i k}^{l}$ via (127) and inserted into $\delta \hat{H}=0$. The ensuing equation could be solved for $\Gamma_{i k}^{l}$ and led to Equation (123). In the third step, the Lagrangian $\hat{H}^{*}$ is taken as a functional of the variables introduced in the first step, i.e., of $\hat{g}^{k l}, \hat{f}^{k l}$ such that in place of Equation (128) the relations

$$
\gamma_{k l}:=\frac{\delta \hat{H} *}{\delta \hat{g}^{k l}}, \quad \phi_{k l}:=\frac{\delta \hat{H} *}{\delta \hat{f}^{k l}} .
$$

hold. Einstein then took "the expression most natural vis-a-vis our present knowledge", i.e., $\hat{H}^{*}=2 \alpha \sqrt{-g}-\frac{\beta}{2} f_{i k} \hat{f}^{i k}$. By using both Equation (127) and Equation (129), Einstein obtained the Einstein-Maxwell equations augmented by a term $-\frac{1}{6} i_{k} i_{l}$ on the side of the energy-momentum tensor of the electromagnetic field and Equation (125) with a changed l.h.s. now reading $-\beta f_{i k}$.

After a field rescaling, he then took a third expression to become his Lagrangian

$$
\bar{H}=\sqrt{-g}\left[R-2 \alpha+\kappa\left(\frac{1}{2} f_{i j} f^{i j}\right)-\frac{1}{\beta} i_{l} i^{l}\right],
$$

where $\alpha$ and $\beta$ are arbitrary constants, and $\kappa$ is the gravitational constant. $i_{l}$ is defined to be proportional to the electromagnetic 4-potential $f_{k}$, i.e., $\frac{1}{\beta} i_{l}=-f_{k}$, and $f_{i j}$ corresponds to $\phi_{i j}, \sqrt{-g} f^{i j}$ to $\hat{f}^{i j}$. After the field equations had been obtained by this longwinded procedure, it became obvious that they could also be derived from $\bar{H}=H$ taken as an "effective" Lagrangian varied with respect to $g_{i k}$ and $f_{i k}$. In Einstein's words: " $R$ is the Riemannian curvature scalar formed from $g_{i j}{ }^{117}$. In the third paper as well, Einstein's desire to create a unified field theory satisfying all

\footnotetext{
115 "Die Theorie vermag also jedenfalls von der Verschiedenheit der Masse der positiven und negativen Elektronen keine Rechenschaft zu geben."

${ }^{116} \mathrm{He}$ exchanged $g_{i k}$ by $\gamma_{i k}, R_{i k}$ by $r_{i k}$.

117 " $R$ bedeutet hierbei den aus den $g_{i j}$ gebildeten RIEMANNschen Krümmungsskalar"
} 
his criteria still was not fulfilled: His equations, again, did not give a singularity-free electron. In a paper on Hilbert's vision of a unified science, Sauer and Majer recently have found out from lectures of Hilbert given in Hamburg and Zürich in 1923, that Hilbert considered Einstein's work in affine theory a return to his own results of 1915 by "[...] a colossal detour via Levi-Civita, Weyl, Schouten, Eddington [...]" [215]. It seems that, in this evaluation, Hilbert was influenced by Einstein's proportionality between the 4-potential and the electrical current which Hilbert had assumed as early as in $1915[161]^{118}$.

\subsubsection{Comments by Einstein's colleagues}

While, in the meantime, mathematicians had taken over the conceptual development of affine theory, some other physicists, including the perpetual pièce de resistance Pauli, kept a negative attitude:

"[...] I now do not at all believe that the problem of elementary particles can be solved by any theory applying the concept of continuously varying field strengths which satisfy certain differential equations to regions in the interior of elementary particles. [...] The quantities $\Gamma_{\nu \alpha}^{\mu}$ cannot be measured directly, but must be obtained from the directly measured quantities by complicated calculational operations. Nobody can determine empirically an affine connection for vectors at neighbouring points if he has not obtained the line element before. Therefore, unlike you and Einstein, I deem the mathematician's discovery of the possibility to found a geometry on an affine connection without a metric as meaningless for physics, in the first place." 119 (Pauli to Eddington on 20 September 1923; [251], pp. 115-119)

Also Weyl, in the 5th edition of Raum-Zeit-Materie ([398], Appendix 4), in discussing "worldgeometric extensions of Einstein's theory", found Eddington's theory not convincing. He criticised a theory that keeps only the connection as a fundamental building block for its lack of a guarantee that it would also house the conformal structure (light cone structure). This is needed for special relativity to be incorporated in some sense, and thus must be an independent fundamental input [405].

Likewise, Eddington himself did not appreciate much Einstein's followership. In Note 14, $\S 100$ appended to the second edition of his book, he laid out Einstein's theory but not without first having warned the reader:

"The theory is intensely formal as indeed all such action-theories must be, and I cannot avoid the suspicion that the mathematical elegance is obtained by a short cut which does not lead along the direct route of real physical progress. From a recent conversation with Einstein I learn that he is of much the same opinion." ([64], pp. 257-261)

In fact, when Eddington's book was translated into German in 1925 [60], Einstein wrote an appendix to it in which he repeated, with minor changes, the results of his last paper on the affine theory. His outlook on the state of the theory now was rather bleak:

\footnotetext{
${ }^{118}$ This proportionality is about the simplest assumption one can make; the equation $\nabla_{l} F^{k l} \sim A^{k}$ corresponds, in the case of the gravitational field, to the equation $R_{i k}=\lambda g_{i k}$ leading to Einstein spaces.

119 “[...] Ich glaube nun überhaupt nicht, dass dieses Problem der elektrischen Elementarteilchen von irgend einer Theorie gelöst werden kann, die den Begriff der kontinuierlich variierenden Feldstärken, die gewissen Differentialgleichungen genügen, auf die Gebiete im Innern der Elementarteilchen anwendet. [...] Die Grössen $\Gamma_{\nu \alpha}^{\mu}$ können nicht direkt gemessen werden, sondern müssen aus den direkt gemessenen Grössen erst durch komplizierte Rechenoperationen gewonnen werden. Niemand kann empirisch einen affinen Zusammenhang zwischen Vektoren in benachbarten Punkten feststellen, wenn er nicht vorher bereits das Linienelement ermittelt hat. Deswegen halte ich im Gegensatz zu Ihnen und Einstein die Erfindung der Mathematiker, dass man auch ohne Linienelement auf einen affinen Zusammenhang eine Geometrie gründen kann, zunächst für die Physik bedeutungslos."
}

Living Reviews in Relativity

http: //www. livingreviews . org/lrr-2004-2 
"For me, the final result of this consideration regrettably consists in the impression that the deepening of the geometrical foundations by Weyl-Eddington is unable to bring progress for our physical understanding; hopefully, future developments will show that this pessimistic opinion has been unjustified." ${ }^{120}$ ([60], p. 371)

An echo of this can be found in Einstein's letter to Besso of 5 June 1925:

"I am firmly convinced that the entire chain of thought Weyl-Eddington-Schouten does not lead to something useful in physics, and I now have found another, physically better founded approach. To me, the quantum-problem seems to require something like a special scalar, for the introduction of which I have found a plausible way." ${ }^{21}$ ([99], p. 204)

This remark shows that Einstein must have taken some notice of Schouten's work in affine geometry. What the "special scalar" was, remains an open question.

\subsubsection{Overdetermination of partial differential equations and elementary particles}

Einstein spent much time in thinking about the "quantum problem", as he confessed to Born:

"I do not believe that the theory will be able to dispense with the continuum. But I fail to succeed in giving my pet idea a tangible form: to understand the quantum-structure through an overdetermination by differential equations." ${ }^{122}$ ([103], pp. 48-49)

In a paper from December 1923, Einstein not only stated clearly the necessary conditions for a unified field theory to be acceptable to him, but also expressed his hope that this technique of "overdetermination" of systems of differential equations could solve the "quantum problem".

"According to the theories known until now the initial state of a system may be chosen freely; the differential equations then give the evolution in time. From our knowledge about quantum states, in particular as it developed in the wake of Bohr's theory during the past decade, this characteristic feature of theory does not correspond to reality. The initial state of an electron moving around a hydrogen nucleus cannot be chosen freely; its choice must correspond to the quantum conditions. In general: not only the evolution in time but also the initial state obey laws." ${ }^{123}$ ([75], pp. 360-361)

He then ventured the hope that a system of overdetermined differential equations is able to determine

\footnotetext{
120 "Für mich besteht das Endergebnis dieser Betrachtung leider in dem Eindruck, dass uns die Weyl-Eddingtonsche Vertiefung der geometrischen Grundlagen keinen Fortschritt der physikalischen Erkenntnis zu bringen vermag; hoffentlich wird die künftige Entwicklung zeigen, dass diese pessimistische Meinung unberechtigt gewesen ist."

121 "Ich bin fest überzeugt, dass die ganze Gedanken-Reihe Weyl-Eddington-Schouten zu nichts physikalisch brauchbarem führt und habe jetzt eine andere Spur gefunden, die mehr physikalisch fundiert ist. Das QuantenProblem scheint mir etwas wie einen besonderen Skalar zu verlangen, für dessen Einführung ich einen plausiblen Weg gefunden habe."

122 "Ich glaube nicht, dass die Theorie das Kontinuum wird entbehren können. Es will mir aber nicht gelingen, meiner Lieblingsidee, die Quantenstruktur aus einer Überbestimmung durch Differentialgleichungen zu verstehen, greifbare Gestalt zu geben."

123 "Nach den bisherigen Theorien kann der Anfangszustand eines Systems frei gewählt werden; die Differentialgleichungen liefern dann die zeitliche Fortsetzung. Nach unserem Wissen über die Quantenzustände, wie es sich insbesondere im Anschluss an die BOHRsche Theorie im letzten Jahrzehnt entwickelt hat, entspricht dieser Zug der Theorie nicht der Wirklichkeit. Der Anfangszustand eines um einen Wasserstoffkern bewegten Elektrons kann nicht frei gewählt werden, sondern diese Wahl muss den Quantenbedingungen entsprechen. Allgemein: nicht nur die zeitliche Fortsetzung, sondern auch der Anfangszustand unterliegt Gesetzen."
} 
"also the mechanical behaviour of singular points (electrons) in such a way that the initial states of the field and of the singular points are subjected to constraints as well. [...] If it is possible at all to solve the quantum problem by differential equations, we may hope to reach the goal in this direction."

We note here Einstein's emphasis on the very special problem of the quantum nature of elementary particles like the electron, as compared to the general problem of embedding matter fields into a geometrical setting.

One of the crucial tests for an acceptable unified field theory for him now was:

"The system of differential equations to be found, and which overdetermines the field, in any case must admit this static, spherically symmetric solution which describes, respectively, the positive and negative electron according to the equations given above [i.e the Einstein-Maxwell equations]." 124

This attitude can also be found in a letter to M. Besso from 5 January 1924:

"The idea I am wrestling with concerns the understanding of the quantum facts; it is: overdetermination of the laws by more field equations than field variables. In such a way, the un-ambiguity of the initial conditions ought to be understood without leaving field theory. [...] The equations of motion of material points (electrons) will be given up totally; their motion ought to be co-determined by the field laws." ${ }^{125}$ ([99], p. 197)

In his answer, Besso asked for more information concerning the quantum aspect of the concept of "overdetermination", because:

"On the one hand, this seems to be connected only formally with a field theory; on the other, it has not yet dawned on me how in this manner something corresponding to the discrete quantum orbits may be reached." ${ }^{126}$ ([99], p. 199)

\footnotetext{
124 "Das gesuchte Gleichungssystem, welches das Feld überbestimmt, muss jedenfalls jene statische, kugelsymmetrische Lösung zulassen, welche gemäss obigen Gleichungen [i.e., the Einstein-Maxwell equations] das positive bzw. negative Elektron beschreibt."

125 "Die Idee, mit der ich mich herumschlage, betrifft das Verstehen der Quantentheorie und heisst: Überbestimmung der Gesetze durch mehr Gleichungen als Feldvariable. So soll die Nichtwillkürlichkeit der Anfangsbedingungen begriffen werden, ohne die Feldtheorie zu verlassen.[...] Die Bewegungsgleichungen materieller Punkte (Elektronen) wird ganz aufgegeben; das motorische Verhalten der letzteren soll durch die Feldgesetze mitbestimmt werden."

126 "Einerseits scheint das nur noch formell etwas mit einer Feldtheorie gemeinsam zu haben; und andererseits schimmert mir noch nicht, wie auf diesem Wege etwas den diskreten Quantenbahnen entsprechendes zu erreichen ist."
} 


\section{Differential Geometry's High Tide}

In the introduction to his book, Struik distinguished three directions in the development of the theory of linear connections [337]:

(1) The generalisation of parallel transport in the sense of Levi-Civita and Weyl. Schouten is the leading figure in this approach [300].

(2) The "geometry of paths" considering the lines of constant direction for a connection - with the proponents Veblen, Eisenhart [122, 114, 115, 373], J. M. Thomas [348], and T. Y. Thomas ${ }^{127}$ [349, 347]. Here, only symmetric connections can appear.

(3) The idea of mapping a manifold at one point to a manifold at a neighbouring point is central (affine, conformal, projective mappings). The names of König [192] and Cartan [29, 302] are connected with this program.

In his assessment, Eisenhart [121] adds to this all the geometries whose metric is

"based upon an integral whose integrand is homogeneous of the first degree in the differentials. Developments of this theory have been made by Finsler, Berwald, Synge, and J. H. Taylor. In this geometry the paths are the shortest lines, and in that sense are a generalisation of geodesics. Affine properties of these spaces are obtained from a natural generalisation of the definition of Levi-Civita for Riemannian spaces." ([121], p. V)

In fact, already in May 1921 Jan Arnoldus Schouten in Delft had submitted two papers classifying all possible connections [297, 296]. In the first he wrote:

"Motivated by relativity theory, differential geometry received a totally novel, simple and satisfying foundation; I just refer to G. Hessenberg's 'Vectorial foundation...', Math. Ann. 78, 1917, S. 187-217 and H. Weyl, Raum-Zeit-Materie, 2. Section, Leipzig 1918 (3. Aufl. Berlin 1920) as well as 'Reine Infinitesimalgeometrie' etc. ${ }^{128}$. [...] In the present investigation all 18 different linear connections are listed and determined in an invariant manner. The most general connection is characterised by two fields of third degree, one tensor field of second degree, and a vector field [...]." ${ }^{129}$ ([297], p. 57)

The fields referred to are the torsion tensor $S_{i j}^{k}$, the tensor of non-metricity $Q_{i j}^{k}$, the metric $g_{i j}$, and the tensor $C_{i j}^{k}$ which, in unified field theory, was rarely used. It arose because Schouten introduced different linear connections for tangent vectors and linear forms. He defined the covariant derivative of a 1-form not by the connection $L_{i j}^{k}$ in Equation (13), but by

$$
\stackrel{+}{\omega}_{i}=\frac{\partial \omega_{i}}{\partial x^{k}}-L_{k i}{ }^{j} \omega_{j}
$$

\footnotetext{
127 Tracy Yerkes Thomas (1899-1983). Born in Alton, Illinois, U.S.A.: Studied mathematics at Princeton University and received his doctorate in 1923. Professor at Princeton, then from 1938-1944 at the University of California in Los Angeles, and since 1944 professor and chairman of the mathematics department at Indiana University in Bloomington, U.S.A.

${ }^{128}$ This is [160, 398] and [397].

129 "Durch die Relativitätstheorie veranlasst, hat die Differentialgeometrie eine ganz neue, einfache und befriedigende Begründung erfahren; ich nenne nur G. Hessenberg 'Vektorielle Begründung...', Math. Ann. 78, 1917, S. 187217 und H. Weyl, Raum-Zeit-Materie, 2. Kap., Leipzig 1918 (3. Aufl. Berlin 1920) sowie 'Reine Infinitesimalgeometrie' etc. [...] In der vorliegenden Untersuchung sind nun alle achtzehn verschiedenen Arten der linearen Übertragung vollständig aufgezählt und in invarianter Weise festgelegt. Die allgemeinste Übertragung wird durch zwei Felder dritten Grades, ein Tensorfeld zweiten Grades und ein Vektorfeld charakterisiert, [...]."
} 
with $\prime L \neq L$. In fact

$$
C_{i j}^{k}:=L_{i j}^{k}-L_{i j}^{k}
$$

In the first paper, Schouten had considered only the special case $C_{i j}^{k}=C_{i} \delta_{j}^{k 130}$.

Furthermore, on p. 57 of [297] we read:

"The general connection for $n=4$ at least theoretically opens the door for an extension of Weyl's theory. For such an extension an invariant fixing of the connection is needed, because a physical phenomenon can correspond only to an invariant expression." 131

Through footnote 5 on the same page we learn the pedagogical reason why Schouten did not use the 'direct' method $[294,336]$ in his presentation, but rather a coordinate dependent formalism ${ }^{132}$ :

"As the results of the present investigation might be of interest for a wider circle of mathematicians, and also for a number of physicists [...]." ${ }^{133}$

At the end of the first paper we can find a section "Eventual importance of the present investigation for physics" (p. 79-81) and the confirmation that during the proofreading Schouten received Eddington's paper ([58], accepted 19 February 1921). Thus, while Einstein and Weyl influenced Eddington, Schouten apparently did his research without knowing of Eddington's idea. Einstein, perhaps, got to know Schouten's work only later through the German translation of Eddington's book where it is mentioned ([60], p. 319), and to which he wrote an addendum, or, more directly, through Schouten's book on the Ricci calculus, Die Grundlehren der Mathematischen Wissenschaften in Einzeldarstellungen, in the same famous yellow series of Springer Verlag [300]. On the other hand, Einstein's papers following Eddington's [77, 74] inspired Schouten to publish on a theory with vector torsion that tried to remedy a problem Einstein had noted in his papers, i.e., that no electromagnetic field could be present in regions of vanishing electric current density. According to Schouten

"[...] we see that the electromagnetic field only depends on the curl of the electric current vector, so that the difficulty arises that the electromagnetic field cannot exist in a place with vanishing current density. In the following pages will be shown that this difficulty disappears when the more general supposition is made that the original deplacement is not necessarily symmetrical." ([300], p. 850)

Schouten criticised Einstein's argument for using a symmetric connection ${ }^{134}$ as unfounded (cf. Equation (15)). He then restricted the generality of his approach; in modern parlance, he did allow for vector torsion only:

"We will not consider the most general case, but the semi-symmetric case in which the alternating part of the parameters has the form:

$$
1 / 2\left(\Gamma_{\mu \lambda}^{\prime} \nu_{\lambda}-\Gamma_{\lambda \mu}^{\prime}{ }^{\nu}\right)=1 / 2\left(S_{\lambda} \delta_{\mu}^{\nu}-S_{\mu} \delta_{\lambda}^{\nu}\right)
$$

in which $S_{\lambda}$ is a general covariant vector." ([298], p. 851)

\footnotetext{
${ }^{130}$ The 18 possibilities were numbered by Schouten as I, ..., VI a-c; he mentioned 5 examples: Einstein (VI c), Hessenberg (VI a), Weyl (IV c) and (II c) (the latter also corresponds to Eddington's choice) as well as König (II a) (cf. also [296]).

131 "Die allgemeinen Übertragungen für $n=4$ eröffnen für die Physik wenigstens theoretisch die Möglichkeit einer Erweiterung der Weylschen Theorie. Für eine solche Erweiterung ist eine invariante Festlegung der Übertragung notwendig, da eine physische Erscheinung nur mit einem invarianten Ausdruck korrespondieren kann."

${ }^{132}$ A summary of Schouten's papers from 1922 is given in [300].

133 "Da die Resultate der vorliegenden Arbeit aber für weitere Kreise von Mathematikern und auch für manche Physiker interessant sein dürften [...]."

${ }^{134}$ Einstein had wished to avoid the distinction between $\stackrel{+}{\nabla}$ and $\bar{\nabla}$.
} 
The affine connection $\Gamma^{\prime}$ can then be decomposed as follows:

$$
\Gamma_{j k}^{\prime l}=\Lambda_{j k}^{l}+S_{[j} \delta_{k]}^{l} .
$$

Hence, besides the covariant derivative $\nabla^{\prime}$ following from use of $\Gamma_{j k}^{\prime}$, in his calculations Schouten also introduced a covariant derivative $\nabla^{*}$ formed with $\Lambda_{j k}{ }^{l}$. Schouten's point of departure for the field equations is Einstein's first Lagrangian $\mathcal{L}=\sqrt{\operatorname{det} K_{i j}}$ and, consequently, his field equations were the same as Einstein's apart from additional terms in vector torsion. Also, Schouten's definition of some of the observables is different; For example, the electromagnetic field tensor unlike in Equation (125) is now

$$
\hat{F}_{k l}=\frac{1}{6}\left(\frac{\partial \hat{\imath}_{k}}{\partial x^{l}}-\frac{\partial \hat{\imath}_{l}}{\partial x^{k}}\right)-\left(\frac{\partial \hat{S}_{k}}{\partial x^{l}}-\frac{\partial \hat{S}_{l}}{\partial x^{k}}\right),
$$

where $i^{k}:=\nabla_{l}^{*} f^{k l}-P_{l} f^{k l}$, and $P_{k}:=-\frac{\partial}{\partial x^{k}}\left(\log \sqrt{-\operatorname{det} K_{i j}}\right)+\Lambda_{l k}^{l}$. On the same topic, Schouten wrote a paper with Friedman in Leningrad [142]. A similar, but less detailed, classification of connections than Schouten's has also been given by Cartan. He relied on the curvature, torsion and homothetic curvature 2-forms ([32], Section III; cf. also Section 2.1.4). In 1925, Eyraud ${ }^{135}$ came back to Schouten's paper [298] and proved that his connection can be mapped projectively and conformally on a Riemannian space [124, 123].

Other mathematicians were also stimulated by Einstein's use of differential geometry in his general relativity and, particularly, by the idea of unified field theory. Examples are Eisenhart and Veblen, both in Princeton, who developed the "geometry of paths" ${ }^{136}$ under the influence of papers by Weyl, Eddington, and Einstein [122, 117, 383]. In Eisenhart's paper, we may read that

"Einstein has said (in Meaning of Relativity) that 'a theory of relativity in which the gravitational field and the electromagnetic field enter as an essential unity' is desirable and recently has proposed such a theory." ([117], pp. 367-368)

and

"His geometry also is included in the one now proposed and it may be that the latter, because of its greater generality and adaptability will serve better as the basis for the mathematical formulation of the results of physical experiments." ([117], p. 369)

The spreading of knowledge about properties of differential geometric objects like connection and curvature took time, however, even in Leningrad. Seven years after Schouten's classification of connections, Fréedericksz of Leningrad - known better for his contributions to the physics of liquid crystals - put forward a classification of his own by using both the connection and the curvature tensor [138].

\footnotetext{
135 Henri Eyraud (1892-1994). Studied mathematics at the University of Paris and received his doctorate in 1926 with a thesis on "Metrical spaces and physico-geometrical theories". From 1930 professor of mathematics at the University of Lyon and director of the Institute of "Financial and Assurance-Sciences". Perhaps he considered his papers on the geometry of unified field theory as a sin of his youth: In Poggendorff, among the 33 papers listed, all are from his later main interest.

${ }^{136}$ The geometry of paths involves a change of connection that preserves the geodesics when vectors are displaced along themselves.
} 


\section{The Pursuit of Unified Field Theory by Einstein and His Collaborators}

\subsection{Affine and mixed geometry}

Already in July 1925 Einstein had laid aside his doubts concerning "the deepening of the geometric foundations". He modified Eddington's approach to the extent that he now took both a nonsymmetric connection and a non-symmetric metric, i.e., dealt with a mixed geometry (metric-affine theory):

"[...] Also, my opinion about my paper which appeared in these reports [i.e., Sitzungsberichte of the Prussian Academy, Nr. 17, p. 137, 1923], and which was based on Eddington's fundamental idea, is such that it does not present the true solution of the problem. After an uninterrupted search during the past two years I now believe to have found the true solution." 137 ([78], p. 414)

As in general relativity, he started from the Lagrangian $\mathcal{L}=\hat{g}^{i k} R_{i k}$, but now with $\hat{g}^{i k}$ and the connection $\Gamma_{k j}^{l}$ being varied separately as independent variables. After some manipulations, the variation with regard to the metric and to the connection led to the following equations:

$$
-\frac{\partial g_{i k}}{\partial x^{l}}+g_{r k} \Gamma_{i l}^{r}+g_{i r} \Gamma_{l k}^{r}+g_{i k} \phi_{l}+g_{i l} \phi_{k}=0, \quad R_{i k}=0,
$$

i.e., $64+16$ equations for the same number of variables. $\phi_{k}$ is an arbitrary covariant vector. The asymmetric $g_{i k}$ is related to $\hat{g}^{l m}$ by

$$
\hat{g}_{i r} \hat{g}^{j r}=\hat{g}_{r i} \hat{g}^{r j}=\delta_{i}{ }^{j}, \quad \hat{g}_{i k}=\frac{g_{i k}}{\sqrt{-g}} .
$$

The three equations (134) and

$$
\frac{\partial \hat{g}^{i k}}{\partial x^{k}}-\frac{\partial \hat{g}^{k i}}{\partial x^{k}}=0, \quad R_{i k}=0,
$$

were the result of the variation. In order to be able to interpret the symmetric part of $g_{i k}$ as metrical tensor and its anti(skew)-symmetric part as the electromagnetic field tensor, Einstein put $\phi_{k}=0$, i.e., overdetermined his system of partial differential equations. However, he cautioned:

"However, for later investigations (e.g., the problem of the electron) it is to be kept in mind that the HAMILTONian principle does not provide an argument for putting $\phi_{k}$ equal to zero." 138

In comparing Equation (134) with $\phi_{k}=0$ and Equation (47), we note that the expression does not seem to correspond to a covariant derivative due to the + sign where a $-\operatorname{sign}$ is required. But this must be due to either a calculational error, or to a printer's typo because in the paper of J. M. Thomas following Einstein's by six months and showing that Einstein's

"new equations can be obtained by direct generalisation of the equations of the gravitational field previously given by him. The process of generalisation consists in abandoning assumptions of symmetry and in adopting a definition of covariant differentiation which is not the usual one, but which reduces to the usual one in case the connection is symmetric." ([346], p. 187)

137 “...] Auch von meiner in diesen Sitzungsberichten (Nr. 17, p. 137 1923) erschienenen Abhandlung, welche ganz auf Eddingtons Grundgedanke basiert war, bin ich der Ansicht, dass sie die wahre Lösung des Problems nicht gibt. Nach unablässigem Suchen in den letzten zwei Jahren glaube ich nun die wahre Lösung gefunden zu haben."

138 "Man wird jedoch für spätere Untersuchungen (z. B. Problem des Elektrons) im Sinne behalten müssen, dass das HAMILTONsche Prinzip für das Verschwinden der $\phi_{k}$ keinen Anhaltspunkt liefert." 
J. M. Thomas wrote Einstein's Equation (134) in the form

$$
g_{i k / l}=g_{i k} \phi_{l}+g_{i l} \phi_{k}, \quad \phi_{l}=-\frac{2}{n-1} \Omega_{r l}^{r},
$$

with $\Omega$ being the skew-symmetric part of the asymmetric connection $H_{i j}{ }^{k}=\Gamma_{(i j)}^{k}+\Omega_{[i j]}^{k}$, and $g_{i j}$ being the symmetric part of the asymmetric metric $h_{i j}=g_{(i j)}+\omega_{[i j]}$. The two covariant derivatives introduced by J. M. Thomas are $g_{i j, k}=\frac{\partial g_{i j}}{\partial x^{k}}-g_{r j} \Gamma_{i k}^{r}-g_{i r} \Gamma_{j k}^{r}$ and $h_{i j / k}=\frac{\partial h_{i j}}{\partial x^{k}}-h_{r j} H_{i k}{ }^{r}-h_{i r} H_{j k}{ }^{r}$. J. M. Thomas then could reformulate Equation (137) in the form

$$
g_{i j / l}=g_{[r i]} \Omega_{j l}^{r}+g_{[i r]} \Omega_{l j}^{r},
$$

and derive the result

$$
g_{i j, l}+g_{j l, i}+g_{l i, j}=0
$$

(see [346], p. 189).

After having shown that his new theory contains the vacuum field equations of general relativity for vanishing electromagnetic field, Einstein then proved that, in a first-order approximation, Maxwell's field equations result cum grano salis: Instead of $F_{i k, l}+F_{l i, k}+F_{k l, i}=0$ he only obtained $\sum \frac{\partial}{\partial x^{l}}\left(F_{i k, l}+F_{l i, k}+F_{k l, i}\right)=0$.

This was commented on in a paper by Eisenhart who showed "more particularly what kind of linear connection Einstein has employed" and who obtained "in tensor form the equations which in this theory should replace Maxwell's equations." He then pointed to some difficulty in Einstein's theory: When identification of the components of the antisymmetric part $\phi_{i j}$ of the metric $a_{i j}=g_{i j}+\phi_{i j}$ with the electromagnetic field is made in first order,

"they are not the components of the curl of a vector as in the classical theory, unless an additional condition is added." ([120], p. 129)

Toward the end of the paper Einstein discussed time-reversal; according to him, by it the sign of the magnetic field is changed, while the sign of the electric field vector is left unchanged ${ }^{139}$. As he wanted to obtain charge-symmetric solutions from his equations, Einstein now proposed to change the roles of the magnetic fields and the electric fields in the electromagnetic field tensor. In fact, the substitutions $\tilde{\mathbf{E}} \rightarrow \tilde{\mathbf{B}}$ and $\tilde{\mathbf{B}} \rightarrow-\tilde{\mathbf{E}}$ leave invariant Maxwell's vacuum field equations (duality transformations) $^{140}$. Already Pauli had pointed to time-reflection symmetry in relation with the problem of having elementary particles with charge $\pm e$ and unequal mass ([246], p. 774).

At first, Einstein seems to have been proud about his new version of unified field theory; he wrote to Besso on 28 July 1925 that he would have liked to present him "orally, the egg laid recently, but now I do it in writing", and then explained the independence of metric and connection in his mixed geometry. He went on to say:

"If the assumption of symmetry ${ }^{141}$ is dropped, the laws of gravitation and Maxwell's field laws for empty space are obtained in first approximation; the antisymmetric part of $\hat{g}^{i k}$ is the electromagnetic field. This is surely a magnificent possibility which likely corresponds to reality. The question now is whether this field theory is consistent

\footnotetext{
${ }^{139}$ Some extended discussion about the transformation of the field components with regard to time-reversal exist, in which two differing points of view are expressed (cf. [170], pp. 91-92, which corresponds to Einstein's view, and [390]).

${ }^{140}$ Einstein's proof that charge-symmetric solutions with the same mass are unavoidable, although to him a rather negative feature of his unified field theories, later was interpreted as Einstein's discovery of the concept of antimatter ([357, 371], p. 78, footnote 44). To me, this seems to be a case of whiggish historical hindsight. According to Bargmann, Einstein's lasting result is that he pointed out the importance of the discrete symmetry operations [8].

${ }^{141}$ i.e., the symmetry of the metrical tensor.
} 
with the existence of quanta and atoms. In the macroscopic realm, I do not doubt its

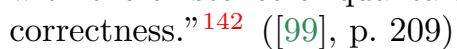

We have noted before that a similar suggestion within a theory with a geometry built from an asymmetric metric had been made, in 1917, by Bach alias Förster.

Yet, in the end, also this novel approach did not convince Einstein. Soon after the publication discussed, he found his argument concerning charge symmetric solutions not to be helpful. The link between the occurrence of solutions with both signs of the charge with time-symmetry of the field equations induced him to doubt, if only for a moment, whether the endeavour of unifying electricity and gravitation made sense at all:

"To me, the insight seems to be important that an explanation of the dissimilarity of the two electricities is possible only if time is given a preferred direction, and if this is taken into account in the definition of the decisive physical quantities. In this, electrodynamics is basically different from gravitation; therefore, the endeavour to melt electrodynamics with the law of gravitation into one unity, to me no longer seems to be justified." 143 [79]

In a paper dealing with the field equations

$$
R_{i k}-\frac{R}{4} g_{i k}=-\kappa T_{i k}
$$

which had been discussed earlier by Einstein [70], and to which he came back now after Rainich ${ }^{144}$ 's insightful paper into the algebraic properties of both the curvature tensor and the electromagnetic field tensor ([263, 264, 265, 266]), Einstein indicated that he had lost hope in the extension of Eddington's affine theory:

"That the equations (140) have received only little attention is due to two circumstances. First, the attempts of all of us were directed to arrive, along the path taken by Weyl and Eddington or a similar one, at a theory melting into a formal unity the gravitational and electromagnetic fields; but by lasting failure I now have laboured to convince myself that truth cannot be approached along this path." ${ }^{145}$ (Einstein's italics; [80], p. 100)

The new field equation was picked up by R. N. Sen of Kalkutta who calculated "the energy of an electric particle" according to it [323].

\footnotetext{
142 "Lässt man die Voraussetzung der Symmetrie fallen, so erhält man in erster Näherung die Gesetze der Gravitation und die Maxwell'schen Feldgesetze für den leeren Raum, wobei der antisymmetrische Teil der $\hat{g}^{i k}$ das elektromagnetische Feld ist. Dies ist doch eine prachtvolle Möglichkeit, die doch der Realität entsprechen dürfte. Nun ist die Frage, ob diese Feldtheorie mit der Existenz der Atome und Quanten vereinbar ist. Im Makroskopischen zweifle ich nicht an ihrer Richtigkeit."

143 "Wesentlich scheint mir die Erkenntnis zu sein, dass eine Erklärung der Ungleichartigkeit der beiden Elektrizitäten nur möglich ist, wenn man der Zeit eine Ablaufrichtung zuschreibt und diese bei der Definition der massgebenden physikalischen Grössen heranzieht. Hierin unterscheidet sich die Elektrodynamik von der Gravitation; deshalb erscheint mir auch das Bestreben, die Elektrodynamik mit dem Gravitationsgesetz zu einer Einheit zu verschmelzen, nicht mehr gerechtfertigt."

144 George Yuri Rainich (1886-1968). Of Russian origin. He studied mathematics at universities in Odessa, Göttingen, and Munich, taking his final exam at the University of Kazan in 1913. He then taught at Kazan and Odessa until 1922, when he came to the United States of America. He was a Johnston Scholar at Johns Hopkins University from 1923-1926 and then Professor of Mathematics at the University of Michigan in Ann Arbor, U.S.A. 145 "Dass die Gleichungen (140) noch wenig Beachtung gefunden haben, liegt an zwei Umständen. Erstens nämlich waren unser aller Bestrebungen darauf gerichtet, auf dem von Weyl und Eddington eingeschlagenen oder einem ähnlichen Weg zu einer Theorie zu gelangen, die das Gravitationsfeld und das elektromagnetische Feld zu einer formalen Einheit verschmilzt; durch mannigfache Misserfolge habe ich mich aber nun zu der Überzeugung durchgerungen, dass man auf diesem Wege der Wahrheit nicht näher kommt."
} 
In the same spirit as the one of his paper, Einstein said good bye to his theory in a letter to Besso on Christmas 1925 in words similar to those in his letter in June:

"Regrettably, I had to throw away my work in the spirit of Eddington. Anyway, I now am convinced that, unfortunately, nothing can be made with the complex of ideas by Weyl-Eddington. The equations

$$
R_{i k}-\frac{1}{4} R g_{i k}=-\kappa T_{i k} \quad \text { electromagnetic }
$$

I take as the best we have nowadays. They are 9 equations for the 14 variables $g_{i k}$ and $\gamma_{i k}$. New calculations seem to show that these equations yield the motion of the electrons. But it appears doubtful whether there is room in them for the quanta." 146 ([99], p. 216)

According to the commenting note by Tonnelat, the 14 variables are given by the 10 components of the symmetric part $g_{(i k)}{ }^{147}$ of the metric and the 4 components of the electromagnetic vector potential "the rotation of which are formed by the $\gamma_{[i k]}$ "148.

But even "the best we have nowadays" did not satisfy Einstein; half a year later, he expressed his opinion in a letter to Besso:

"Also, the equation put forward by myself ${ }^{149}$,

$$
R_{i k}=g_{i k} f_{l m} f^{l m}-\frac{1}{2} f_{l} f_{k m} g^{l m}
$$

gives me little satisfaction. It does not allow for electrical masses free from singularities. Moreover, I cannot bring myself to gluing together two items (as the l.h.s. and the r.h.s. of an equation) which from a logical-mathematical point of view have nothing to do with each other." 150 ([99], p. 230)

\subsection{Further work on (metric-) affine and mixed geometry}

Research on affine geometry as a frame for unified field theory was also carried on by mathematicians of the Princeton school. Thus J. M. Thomas, after having given a review of Weyl's, Einstein's, and Schouten's approaches, said about his own work:

\footnotetext{
146 "Meine Arbeit im Sinne Eddington's habe ich leider verwerfen müssen. Ueberhaupt bin ich jetzt überzeugt, dass mit dem Weyl-Eddington'schen Gedanken-Komplex leider nichts zu machen ist. Ich halte die Gleichung

$$
R_{i k}-\frac{1}{4} R g_{i k}=-\kappa T_{i k} \quad \text { elektromagnetisch }
$$

[cf. Equation (140)] für das beste, was wir heute haben. Es sind 9 Gleichungen für die 14 Grössen $g_{i k}$ und $\gamma_{i k}$. Aus den neuen Rechnungen scheint sich zu ergeben, dass diese Gleichungen die Bewegung der Elektronen liefern. Aber es erscheint zweifelhaft, ob die Quanten darin Platz haben."

${ }^{147}$ It remains unclear how these $\gamma_{[i k]}$ are embedded into the theory, possibly in the sense of Rainich. Einstein's paper practically excludes that they form the antisymmetric part of an asymmetric metric tensor.

148 "dont les $\gamma_{[i k]}$ forment le rotationel"

${ }^{149}$ Einstein had a wrong factor: $\frac{1}{2}$ instead of $\frac{1}{4}$.

150 "Auch die ja von mir selbst aufgestellte Gleichung
}

$$
R_{i k}=g_{i k} f_{l m} f^{l m}-\frac{1}{2} f_{l} f_{k m} g^{l m}
$$

befriedigt mich wenig. Sie lässt keine singularitätenfreien elektrischen Massen zu. Ferner kann ich mich nicht dazu entschliessen, zwei Sachen zusammenzuleimen (wie die rechte und die linke Seite einer Gleichung), die logischmathematisch nichts miteinander zu schaffen haben." 
"I show in the present paper that his [Einstein's] new equations can be obtained by a direct generalisation of the equations of the gravitational field previously given by $\operatorname{him}\left[g_{i j ; k}=0 ; R_{i j}=0\right]$. [... In the final section I show that the adoption of the ordinary definition of covariant differentiation leads to a geometry which includes as a special case that proposed by Weyl as a basis for the electric theory; further that the asymmetric connection for this special case is of the type adopted by Schouten for the geometry at the basis of his electric theory." ([346], p. 187)

We met J. M. Thomas' paper before in Section 6.1.

During the period considered here, a few physicists followed the path of Eddington and Einstein. One who had absorbed Eddington's and Einstein's theories a bit later was Infeld ${ }^{151}$ of Warsaw ${ }^{152}$. In January 1928, he followed Einstein by using an asymmetric metric the symmetric part $\gamma_{i k}$ of which stood for the gravitational potential, the skew-symmetric part $\phi_{i k}$ for the electromagnetic field. However, he set the non-metricity tensor (of the symmetric part $\gamma$ of the metric) $Q_{i j}{ }^{k}=0$, and assumed for the skew-symmetric part $\phi$,

$$
\nabla_{l} \phi_{i j}=J_{i j l}
$$

with an arbitrary tensor $J_{i j l}$. The electric current vector then is defined by $J^{i}=J_{l}^{i l}$ where the indices, as I assume, are moved with $\gamma_{i k}$. In a weak-field approximation for the metric, Infeld's connection turned out to be $L_{i k}^{l}=\left\{\begin{array}{c}l \\ i k\end{array}\right\}+\frac{1}{2}\left(\phi_{i, k}{ }^{l}+\phi_{k, i}{ }^{l}+\delta^{l s} \phi_{i k, s}\right)$. For field equations Infeld postulated the (generalised) Einstein field equations in empty space, $K_{i j}=0$. He showed that, in first approximation, he got what is wanted, i.e., Einstein's and Maxwell's equations [166].

Three months later, Infeld published a note in Comptes Rendus of the Parisian Academy in which he now presented the exact connection as

$$
L_{i k}^{l}=\left\{{ }_{i k}^{l}\right\}+\frac{\alpha}{2}\left(\phi_{i, k}^{l}+\phi_{k, i}^{l}+g^{l s} \phi_{i k, s}\right),
$$

where $\alpha$ is "an extremely small numerical factor". By neglecting terms $\sim \alpha^{2}$ he could gain both Einstein's field equation in empty space (94) and Maxwell's equation, if the electric current vector is identified with $\alpha^{-1}\left(L_{i l}^{l}-L_{l i}^{l}\right)$. Thus, he is back at vector torsion treated before by Schouten [298].

The Japanese physicist Hattori embarked on a metric-affine geometry derived purely from an asymmetric metrical tensor $h_{i k}=g_{(i k)}+f_{[i k]}$. He defined an affine connection

$$
L_{i k}^{j}=\left\{\begin{array}{l}
j \\
i k
\end{array}\right\}+g^{j l}\left(f_{l i, k}+f_{l i, k}-f_{i k, l}\right),
$$

where $g^{i l} g_{l k}=\delta_{k}{ }_{k}$, and the Christoffel symbol is formed from g. The electromagnetic field was not identified with $f_{i k}$ by Hattori, but with the skew-symmetric part of the (generalised) Ricci tensor formed from $L_{i k}{ }^{j}$. By introducing the tensor $f_{i j k}:=\frac{\partial f_{i j}}{\partial x^{k}}+\frac{\partial f_{j k}}{\partial x^{i}}+\frac{\partial f_{k i}}{\partial x^{j}}$, he could write the (generalised) Ricci tensor as

$$
K_{i k}=R_{i k}+\frac{1}{4} f_{i m}^{n} f_{k n}^{m}-\nabla_{l} f_{i k}^{l}
$$

where the covariant derivative $\nabla$ is formed with the Levi-Civita connection of $g_{i j}$. The electromagnetic field tensor $F_{i k}$ now is introduced through a tensor potential by $F_{i k}:=\nabla_{l} f_{i k} l$ and leads to half of "Maxwell's" equations. In the sequel, Hattori started from a Lagrangian $\mathcal{L}=\left(g^{i k}+\alpha^{2} F^{i k}\right) K_{i k}$

\footnotetext{
151 Leopold Infeld (1889-1968). Born in Cracow, Poland. Studied at the University of Cracow and received his doctorate in 1923. After teaching in Lwow/Lemberg, he became professor of applied mathematics at the University of Toronto in 1938. Worked on unitary field theory and quantum electrodynamics, with van der Waerden on spinors, worked with Born on non-linear electrodynamics, and with Einstein on equations of motion ("EIH paper").

${ }^{152}$ For the correspondence between Einstein and Infeld, cf. J. Stachel's essay in [330], pp. 477-497.
} 
with the constant $\alpha^{2}$ and varied, alternatively, with respect to $g_{i j}$ and $f_{i j}$. He could write the field equations in the form of Einstein's, with the energy-momentum tensor of the electromagnetic field $F_{i k}$ and a "matter" tensor $M^{i k}$ on the r.h.s., $M^{i k}$ being a complicated, purely geometrical quantity depending on $K_{i k}, K, f_{i k l}$, and $F_{i k l}$. $F_{i k l}$ is formed from $F_{i k}$ as $f_{i k l}$ from $f_{i k}$. From the variation with regard to $f_{i k}$, in addition to Maxwell's equation, a further field equation resulted, which could be brought into the form

$$
F^{i k}=\frac{2}{3} \nabla_{l} F^{i k l}
$$

i.e., $f_{i k l} \sim F_{i k l}$. Hattori's conclusion was:

"The preceding equation shows that electrical charge and electrical current are distributed wherever an electromagnetic field exists." ${ }^{153}$

Thus, the same problem obtained as in Einstein's theory: A field without electric current or charge density could not exist $[155]^{154}$.

Infeld quickly reacted to Hattori's paper by noting that Hattori's voluminous calculations could be simplified by use of Schouten's Equation (39) of Section 2.1.2. As in Hattori's theory two connections are used, Infeld criticised that Hattori had not explained what his fundamental geometry should be: Riemannian or non-Riemannian? He then gave another example for a theory allowing the identification of the electromagnetic field tensor with the antisymmetric part of the Ricci tensor: He displayed again the well-known connection with vector torsion used by Schouten [298] without referring to Schouten's paper [165]. He also claimed that Hattori's Equation (145) is the same as the one that had been deduced from Eddington's theory by Einstein in the Appendix to the German translation of Eddington's book ([60], p. 367). All in all, Infeld's critique tended to deny that Hattori's theory was more general than Einstein's, and to point out

"that the problem of generalising the theory of relativity cannot be solved along a purely formal way. At first, one does not see how a choice can be made among the various nonRiemannian geometries providing us with the gravitational and Maxwell's equations. The proper world geometry which ought to lead to a unified theory of gravitation and electricity can only be found by an investigation of its physical content." 155 ([165], p. 811)

Infeld could as well have applied this admonishment to his own unified field theory discussed above. Perhaps, he became irritated by comparing his expression for the connection (142) with Hattori's (145).

In June 1931, von Laue submitted a paper of the Genuese mathematical physicist Paolo Straneo to the Berlin Academy [331]. In it Straneo took note of Einstein's teleparallel geometry, but decided to take another route within mixed geometry; he started with a symmetric metric and the asymmetric connection

$$
L_{i k}^{j}=\left\{\begin{array}{l}
j \\
i k
\end{array}\right\}+2 \delta_{i}^{j} \psi_{k}
$$

with both non-vanishing curvature tensor $K_{j k l}^{i}=R_{j k l}^{i}+2 \delta_{j}^{i}\left(\frac{\partial \psi_{l}}{\partial x^{k}}-\frac{\partial \psi_{k}}{\partial x^{l}}\right)$ and torsion $S_{i k}{ }^{j}=2 \delta^{j}{ }_{[i} \psi_{k]}$. Thus, Straneo suggested a unified field theory with only vector torsion as Schouten had done 8

\footnotetext{
153 "Die obige Gleichung zeigt, dass sich die elektrische Ladung und der elektrische Strom überall verteilen, wo das elektromagnetische Feld existiert."

${ }^{154}$ I have not yet been able to read the contributions from other Japanese authors [197, 164, 182].

155 "das Problem der Verallgemeinerung der Relativitätstheorie nicht auf rein formalem Wege gelöst werden kann. Man sieht zunächst nicht, wie die Wahl zwischen den verschiedenen nicht-Riemannschen Geometrien, die uns die Gravitation- und die Maxwellschen Gleichungen ergeben, zu treffen ist. Die eigentliche Weltgeometrie, die zu einer einheitlichen Theorie von Gravitation und Elektrizität führen soll, kann nur durch Untersuchung ihres physikalischen Inhalts gefunden werden."
} 
years earlier $[298,142])$ without referring to him. The field equations Straneo wrote down, i. e.

$$
K_{i k}-\frac{1}{2} K g_{i k}=-\kappa T_{i k}+\Psi_{i k}
$$

where $T_{i k}$ is the symmetric and $\Psi_{i k}$ the antisymmetric part of the 1.h.s., do not fulfill Einstein's conception of unification: Straneo kept the energy-momentum tensor of matter as an extraneous object (including the electromagnetic field) as well as the electric current vector. The antisymmetric part of (147) just is $\Psi_{i k}=\left(\frac{\partial \psi_{l}}{\partial x^{k}}-\frac{\partial \psi_{k}}{\partial x^{l}}\right)$; thus $\Psi_{i k}$ is identified with the electromagnetic field tensor, and the electric current vector $J^{i}$ defined by $\Psi_{l}^{i l}=J^{i}$. Straneo wrote further papers on the subject [332, 333].

By a remark of Straneo, that auto-parallels and geodesics have to be distinguished in an affine geometry, the Indian mathematician Kosambi ${ }^{156}$ felt motivated to approach affine geometry from the system of curves solving $\ddot{x}^{i}+\alpha^{i}(x, \dot{x}, t)$ with an arbitrary parameter $t$. He then defined two covariant "vector-derivations" along an arbitrary curve and arrived at an (asymmetric) affine connection. By this, he claimed to have made superfluous the five-vectors of Einstein and Mayer $^{157}$ [107]. This must be read in the sense that he could obtain the Einstein-Mayer equations from his formalism without introducing a connecting quantity leading from the space of 5-vectors to space-time [195].

Einstein, in his papers, did not comment on the missing metric compatibility in his theory and its physical meaning. Due to this complication - for example even a condition of metric compatibility would not have the physical meaning of the conservation of the norm of an angle between vectors under parallel transport, and the further difficulty that much of the formalism was very clumsy to manipulate; essential work along this line was done only much later in the 10940s and 1950s (Einstein, Einstein and Strauss, Schrödinger, Lichnerowicz, Hlavaty, Tonnelat, and many others). In this work a generalisation of the equation for metric compatibility, i.e., Equation (47), will play a central role. The continuation of this research line will be presented in Part II of this article.

\subsection{Kaluza's idea taken up again}

\subsubsection{Kaluza: Act I}

Einstein became interested in Kaluza's theory again due to O. Klein's paper concerning a relation between "quantum theory and relativity in five dimensions" (see Klein 1926 [185], received by the journal on 28 April 1926). Einstein wrote to his friend and colleague Paul Ehrenfest on 23 August 1926: "Subject Kaluza, Schroedinger, general relativity", and, again on 3 September 1926: "Klein's paper is beautiful and impressive, but I find Kaluza's principle too unnatural." However, less than half a year later he had completely reversed his opinion:

\footnotetext{
156 Damodar D. Kosambi (1907-1966). Of Indian origin; born in Goa he moved to America in 1918 with his learned father and graduated from Harvard University in 1926 in mathematics, history and languages. Taught at the Muslim University of Aligarh and, from 1932, at Ferguson College, Pune. Mathematician, historian, and Sanskrit scholar.

157 Walther Mayer (1887-1948). Studied mathematics at the Federal Institute of Technology in Zürich and at the University of Vienna where he wrote his dissertation and became a Privatdozent (lecturer) with the title "professor". He had made himself a name in topology ("Mayer-Vietoris sequences"), and worked also in differential geometry (well-known textbook "Duschek-Mayer" on differential geometry). In 1929 he became Einstein's assistant with the explicit understanding that he work with him on distant parallelism. It seems that Mayer was appreciated much by Einstein and, despite being in his forties, did accept this role as a collaborator of Einstein. After coming to Princeton with Einstein in 1933, he got a position at the Mathematical Institute of Princeton University and became an associate of the Institute for Advanced Study. Wrote a joint paper with T. Thomas on "Field of parallel vectors in nonanalytic manifolds in the large." Mayer died in 1948.
}

Living Reviews in Relativity

http: //www. livingreviews.org/lrr-2004-2 
"It appears that the union of gravitation and Maxwell's theory is achieved in a completely satisfactory way by the five-dimensional theory (Kaluza-Klein-Fock)." (Einstein to H. A. Lorentz, 16 February 1927)

On the next day (17 February 1927), and ten days later Einstein was to give papers of his own in front of the Prussian Academy in which he pointed out the gauge-group, wrote down the geodesic equation, and derived exactly the Einstein-Maxwell equations - not just in first order as Kaluza had done [81, 82]. He came too late: Klein had already shown the same before [185]. Einstein himself acknowledged indirectly that his two notes in the report of the Berlin Academy did not contain any new material. In his second communication, he added a postscript:

"Mr. Mandel brings to my attention that the results reported by me here are not new.

The entire content can be found in the paper by O. Klein." 158

He then referred to the papers of Klein [185, 186] and to "Fochs Arbeit" which is a paper by Fock $^{159} 1926$ [130], submitted three months later than Klein's paper. That Klein had published another important clarifying note in Nature, in which he closed the fifth dimension, seems to have escaped Einstein ${ }^{160}$ [184]. Unlike in his paper with Grommer, but as in Klein's, Einstein, in his notes, applied the "sharpened cylinder condition", i.e., dropped the scalar field. Thus, the three of them had no chance to find out that Kaluza had made a mistake: For $g_{55} \neq$ const., even in first approximation the new field will appear in the four-dimensional Einstein-Maxwell equations ([145], p. 5).

Mandel $^{161}$ of Leningrad was not given credit by Einstein although he also had rediscovered by a different method some of O. Klein's results [216]. In a footnoote, Mandel stated that he had learned of Kaluza's (whom he spelled "Kalusa") paper only through Klein's article. He started by embedding space-time as a hypersurface $x^{5}=$ const. into $M_{5}$, and derived the field equations in space-time by assuming that the five-dimensional curvature tensor vanishes; by this procedure he obtained also a matter-energy tensor "closely linked to the second fundamental form of this hypersurface". From the geodesics in $M_{5}$ he derived the equations of motion of a charged point particle. One of the two additional terms appearing besides the Lorentz force could be removed by a weakness assumption; as to the second, Mandel opinioned

"that the experimental discovery of the second term appears difficult, yet perhaps not entirely impossible." ([216], p. 145)

As to Fock's paper, it is remarkable because it contains, in nuce, the coupling of the Schrödinger wave function $\psi$ and the electromagnetic potential by the gauge transformation $\psi=\psi_{0} e^{2 \pi i p / h}$, where $h$ is Planck's constant and $p$ "a new parameter with the unit of the quantum of action" [130]. In Fock's words:

"The importance of the additional coordinate parameter $p$ seems to lie in the fact that it causes the invariance of the equations [i.e., the relativistic wave equations] with respect to addition of an arbitrary gradient to the 4-potential." ${ }^{162}$ ([130], p. 228)

\footnotetext{
158 "Herr Mandel macht mich darauf aufmerksam, dass die von mir hier mitgeteilten Ergebnisse nicht neu sind. Der ganze Inhalt findet sich in der Arbeit von O. Klein."

159 Vladimir Aleksandrovich Fo(c)k (1898-1974). Born in St. Petersburg (renamed later Petrograd and Leningrad). Studied at Petrograd University and spent his whole carrier at this University. Member of the USSR Academy of Sciences. Fundamental contributions to quantum theory (Fock space, Hartree-Fock method); also worked in and defended general relativity.

${ }^{160}$ The correspondence is taken from Pais [241] who, in his book, expresses his lack of understanding as to why Einstein published these two papers at all.

161 Heinrich Mandel (1898- ). From 1928 lecturer at the University of Leningrad, and from 1931 research work at the Physics Institute of this university.

162 "Die Bedeutung des überzähligen Koordinatenparameters scheint nämlich gerade darin zu liegen, dass er die Invarianz der Gleichungen [i.e., the relativistic wave equations] in bezug auf die Addition eines beliebigen Gradienten zum Viererpotential bewirkt."
} 
Fock derived the general relativistic wave equation and the equations of motion of a charged point particle; the latter is identified with the null geodesics of $M_{5}$. Neither Mandel nor Fock used the "sharpened cylinder condition" (110).

A main motivation for Klein was to relate the fifth dimension with quantum physics. From a postulated five-dimensional wave equation

$$
a^{i k}\left(\frac{\partial^{2} U}{\partial x^{i} \partial x^{k}}-\left\{\begin{array}{c}
i k \\
r
\end{array}\right\} \frac{\partial U}{\partial x^{r}}\right)=0, \quad i, k,=1, \ldots, 5
$$

and by neglecting the gravitational field, he arrived at the four-dimensional Schrödinger equation after insertion of the quantum mechanical differential operators $-\frac{i h}{2 \pi} \frac{\partial}{\partial x^{i}}$. It was Klein's papers and the magical lure of a link between classical field theory and quantum theory that raised interest in Kaluza's idea - seven years after Kaluza had sent his manuscript to Einstein. Klein acknowledged Mandel's contribution in his second paper received on 22 October 1927, where he also gave further references on work done in the meantime, but remained silent about Einstein's papers [189]. Likewise, Einstein did not comment on Klein's new idea of "dimensional reduction" as it is now called and which justifies Klein's name in the "Kaluza-Klein" theories of our time. By this, the reduction of five-dimensional equations (as e.g., the five-dimensional wave equation) to four-dimensional equations by Fourier decomposition with respect to the new 5th spacelike coordinate $x^{5}$, taken as periodic with period $L$, is understood:

$$
\psi\left(x, x^{5}\right)=\frac{1}{\sqrt{L}} \Sigma_{n} \psi_{n}(x), e^{i n x^{5} / R^{5}}
$$

with an integer $n$. Klein had only the lowest term in the series. The 5 th dimension is assumed to be a circle, topologically, and thus gets a finite linear scale: This is at the base of what now is called "compactification". By adding to this picture the idea of de Broglie waves, Klein brought in Planck's constant and determined the linear scale of $x^{5}$ to be unmeasurably small $\left(\sim 10^{-30}\right)$. From this, the possibility of "forgetting" the fifth dimension arose which up to now has not been observed.

In his papers, Einstein took over Klein's condition $g_{55}=1$, which removed the additional scalar field admitted by the theory. It was Reichenbächer who apparently first tried to perform the projection into space-time of the most general five-dimensional metric, and without using the cylinder condition (109):

"Now, a rather laborious calculation of the five-dimensional curvature quantities in terms of a four-dimensional submanifold contained in it has shown to me also in the general case $\left(g_{55} \neq\right.$ const., dependence of the components of the $f u n d a m e n t a l$ [tensor] of $x^{5}$ is admitted) that the $\mathrm{ch}$ a r a c t e r is t i c properties of the field equations are then conserved as well, i.e., they keep the form

$$
R^{i k}-\frac{1}{2} g^{i k} R=T^{i k}, \quad \frac{\partial \sqrt{g} F^{i k}}{\partial x^{k}}=s^{i},
$$

only the $T^{i k}$ contain further terms besides the electromagnetic energy tensor $S^{i k}$, and the quantities collected in $s^{i}$ do not vanish. [...] The appearance of the new terms on the right hand sides could even be welcomed in the sense that now the field equations are obtained not only for a field point free of matter and charge ${ }^{163}$."164 ([276], p. 426)

\footnotetext{
${ }^{163} \mathrm{My}$ italics.

164 "Nun hat mir die allerdings reichlich mühsehlige Umrechnung der fünfdimensionalen Krümmungsgrössen auf eine in ihr enthaltene vierdimensionale Untermannigfaltigkeit auch im allgemeinen Falle ( $g_{55} \neq$ const., Abhängigkeit der Fund a m e n t a lkomponenten auch von $x^{5}$ zugelassen) gezeigt, dass die we e $\mathrm{nt}$ liche $\mathrm{n}$ Eigenschaften
} 
Here, in nuce, is already contained what more than a decade later Einstein and Bergmann worked out in detail [102].

It is likely that Reichenbächer had been led to this excursion into five-dimensional space, an idea which he had rejected before as unphysical, because his attempt to build a unified field theory in space-time through the ansatz for the metric $\gamma_{i k}=g_{i k}-\epsilon^{2} \phi_{i} \phi_{k}$ with $\phi_{k}$ the electromagnetic 4-potential, had failed. Beyond incredibly complicated field equations nothing much had been gained [275]. Reichenbächer's ansatz is well founded: As we have seen in Section 4.2, due to the violation of covariance in $M_{5}, \gamma_{i k}$ transforms as a tensor under the reduced covariance group.

Even L. de Broglie became interested in Kaluza's "bold but very beautiful theory" and rederived Klein's results his way [46], but not without getting into a squabble with Klein, who felt misunderstood $[188,47]$. He also suggested that one should not accept the cylinder condition, a suggestion looked into by Darrieus who introduced an electrical 5-potential and 5-current, and deduced Maxwell's equations from the five-dimensional homogeneous wave equation and the fivedimensional equation of continuity [43].

In 1929 Mandel tried to "axiomatise" the five-dimensional theory: His two axioms were the cylinder condition (109) and its sharpening, Equation (110). He then weakened the second assumption by assuming that "an objective meaning does not rest in the $g_{i k}$ proper, but only in their quotients", an idea he ascribed to O. Klein and Einstein. He then discussed conformally invariant field equations, and tried to relate them to equations of wave mechanics [220].

Klein's lure lasted for some years. In 1930, N. R. Sen claimed to have investigated the "Keplerproblem for the five-dimensional wave equation of Klein". What he did was to calculate the energy levels of the hydrogen atom (as a one particle-system) with the general relativistic wave equation in space-time (148) with $a^{i k}=\gamma^{i k}$, where $\gamma^{i k}=g_{i k}+\alpha^{2} \gamma_{55}$ is the metric on space-time following from the 5-metric $\gamma_{\alpha \beta}$ by $d x^{5}=0$. For $g_{i k}$ he took the Reissner-Nordström solution and did not obtain a discrete spectrum [324]. He continued his approach by trying to solve Schrödinger's wave equation [325]

$$
g^{i k}\left(\frac{\partial^{2} u}{\partial x^{i} \partial x^{k}}-\left\{\begin{array}{c}
i k \\
r
\end{array}\right\} \frac{\partial u}{\partial x^{r}}\right)=-\frac{4 \pi^{2}}{h^{2}} m_{0}^{2} c^{2} u
$$

Presently, the different contributions of Kaluza and O. Klein are lumped together by most physicists into what is called "Kaluza-Klein theory". An early criticism of this unhistorical attitude has been voiced in [210].

\subsubsection{Kaluza: Act II}

Four years later, Einstein returned to Kaluza's idea. Perhaps, he had since absorbed Mandel's ideas which included a projection formalism from the five-dimensional space to space-time [216, 217, 218, 219].

In a paper with his assistant Mayer, Einstein now presented Kaluza's approach in the form of an implicit projective four-dimensional theory, although he did not mention the word "projective" [107]:

der Feldgleichungen auch dann erhalten bleiben, d.h. diese behalten die Gestalt:

$$
R^{i k}-\frac{1}{2} g^{i k} R=T^{i k}, \quad \frac{\partial \sqrt{g} F^{i k}}{\partial x^{k}}=s^{i}
$$

nur enthalten die $T^{i k}$ ausser den Komponenten $S^{i k}$ des elektromagnetischen Energietensors noch weitere Glieder, und die zu $s^{i}$ zusammengefassten Grössen verschwinden nicht. [...] Man könnte sogar das Auftreten der neuen Glieder auf den rechten Seiten von dem Standpunkt aus begrüssen, dass nunmehr die Feldgleichungen nicht nur für einen materie- und ladungsfreien Feldpunkt geliefert werden." 
"Psychologically, the theory presented here connects to Kaluza's well-known theory; however, it avoids extending the physical continuum to one of five dimensions." 165

In the eyes of Einstein, by avoiding the artificial cylinder condition (109), the new method removed a serious objection to Kaluza's theory.

Another motivation is also put forward: The linearity of Maxwell's equations "may not correspond to reality"; thus, for strong electromagnetic fields, Einstein expected deviations from Maxwell's equations. After a listing of all the shortcomings of Kaluza's theory, the new approach is introduced: At every event a five-dimensional vector space $V_{5}$ is affixed to space-time $V_{4}$, and "mixed" tensors $\gamma_{\iota}{ }^{k}$ are defined linking the tangent space of space-time $V_{4}$ with a $V_{5}$ such that

$$
g_{\iota \kappa} \gamma_{l}^{\iota} \gamma_{m}^{\kappa}=g_{l m}
$$

where $g_{l m}$ is the metric tensor of $V_{4}$, and $g_{\iota \kappa}$ a non-singular, symmetric tensor on $V_{5}$ with $\iota, \kappa=$ $1, \ldots, 5$, and $k, l=1, \ldots, 4^{166}$. Indices are raised and lowered with the metrics of $V_{5}$ or $V_{4}$, respectively. There exists a "preferred direction of $V_{5}$ " defined by $\gamma_{\iota}{ }^{k} A^{\iota}=0$, and which is the normal to a "preferred plane" $\gamma_{\iota}{ }^{k} \omega_{k}=0^{167}$. A consequence then is

$$
\gamma_{\sigma k} \gamma^{\tau k}=\gamma_{\sigma}^{k} \gamma_{k}^{\tau}=\delta_{\sigma}^{\tau}-A_{\sigma} A^{\tau} \text {. }
$$

A covariant derivative for five-vectors in $V_{4}$ is defined with a "three-index-symbol" $\Gamma_{\pi l}^{\iota}$ with two indices in $V_{5}$, and one in $V_{4}$ standing in for the connection coefficients:

$$
\stackrel{+}{\nabla}_{l} X^{\iota}=\frac{\partial X^{\iota}}{\partial x^{l}}+\Gamma_{\pi}^{\iota} X^{\pi} .
$$

The covariant derivative of 4 -vectors is defined as usual,

$$
\nabla_{l} X^{i}=\frac{\partial X^{i}}{\partial x^{l}}+\left\{\begin{array}{c}
i \\
j l
\end{array}\right\} X^{j}
$$

where $\left\{\begin{array}{c}i \\ j l\end{array}\right\}$ is calculated from the metric of $V_{4}$ as given in Equation (149). Both covariant derivatives are abbreviated by the same symbol $A_{; k}$. The covariant derivative of tensors with both indices referring to $V_{5}$ and those referring to $V_{4}$, is formed correspondingly. In this context, Einstein and Mayer mention an extension of absolute differential calculus by "WAERDEN and BARTOLOTTI" without giving any reference to their respective papers. They may have had in mind van der Waerden's [368] and Bortolotti ${ }^{168}$ 's [24] papers. The autoparallels of $V_{5}$ lead to the exact equations of motion of a charged particle, not the geodesics of $V_{4}$.

Einstein and Mayer made three basic assumptions:

$$
\begin{aligned}
g_{\iota \kappa ; l} & =0, \\
\gamma_{\iota ; l}^{k} & =A^{\iota} F_{k l}, \\
F_{k l} & =-F_{l k},
\end{aligned}
$$

where $A^{\iota}$ is the preferred direction and $F_{k l}$ an arbitrary 2-form, later to be interpreted as the electromagnetic field tensor. From them $A_{\sigma ; l}=\gamma_{\sigma}{ }^{k} F_{l k}$ follows. They also noted that a symmetric

\footnotetext{
165 "Die hier dargestellte Theorie knüpft psychologisch an die bekannte Theorie von KALUZA an, vermeidet es aber, das physikalische Kontinuum zu einem solchen von fünf Dimensionen zu erweitern."

166 Greek indices run from 1 to 5 , Latin indices from 1 to 4 .

${ }^{167}$ In special coordinates, $A^{\iota} \stackrel{*}{=} \delta_{5}^{\iota}$.

168 Enea Bortolotti (1896-1934). Born in Rome. After a break during the First World War, he received his Ph.D. in 1920 at Pisa; he was particularly influenced by L. Bianchi. After teaching at the medical school, he became professor of geometry at the Univerity of Cagliari in 1928. From there he moved on to the same position at the University of Florence in 1934. Despite his premature death, Bortolotti published about a hundred papers, notably in differential geometry.
} 
tensor $F_{k l}$ could have been interpreted as the second fundamental form, and the formalism would then be the same as local isometric embedding of $V_{4}$ into $V_{5}$.

Einstein and Mayer introduced what they called "Fünferkrümmung" (5-curvature) via the three-index symbol given above by

$$
P_{\iota k l}^{\sigma}=\partial_{k} \Gamma_{\iota l}^{\sigma}-\partial_{l} \Gamma_{\iota k}^{\sigma}+\Gamma_{\tau k}^{\sigma} \Gamma_{\iota l}^{\tau}-\Gamma_{\tau l}^{\sigma} \Gamma_{\iota k}^{\tau}
$$

It is related to the Riemannian curvature $R_{m l k}^{r}$ of $V_{4}$ by

$$
P_{\iota k l}^{\sigma} \gamma_{\sigma m}=A_{\iota}\left(F_{m k ; l}-F_{m l ; k}\right)+\gamma_{\iota r}\left(R_{m l k}^{r}+F_{m k} F_{l}^{r}-F_{m l} F_{k}^{r}\right),
$$

and

$$
P_{\iota k l}^{\sigma} A_{\sigma}=\gamma_{\iota r}\left(F_{k ; l}^{r}-F_{l ; k}^{r}\right) \text {. }
$$

From (154), by transvection with $\gamma^{\tau k}$, the 5 -curvature itself appears:

$$
P_{\iota k l}^{\tau}=\gamma_{\iota r} A^{\tau}\left(F_{k ; l}^{r}-F_{l ; k}^{r}\right)+\gamma^{\tau r} A_{\iota}\left(F_{r k ; l}-F_{r l ; k}\right)+\gamma_{\iota r} \gamma^{\tau s}\left(R_{s l k}^{r}+F_{s k} F_{l}^{r}-F_{s l} F_{k}^{r}\right) .
$$

By contraction, $P_{\iota k}:=\gamma_{\tau}^{r} P_{\iota r k}^{\tau}$ and $P:=\gamma^{\iota k} P_{\iota k}$. Two new quantities are introduced:

(1) $U_{\iota k}:=P_{\iota k}-\frac{1}{4}(P+R)$, where $\mathrm{R}$ is the Ricci scalar of the Riemannian curvature tensor of $V_{4}$, and

(2) the tensor $N_{k l m}:=F_{\{k l ; m\}}{ }^{169}$.

It turns out that $P=R-F_{k p} F^{k p}$.

The field equations put forward in the paper by Einstein and Mayer now are

$$
U_{\iota k}=0, \quad N_{k l m}=0,
$$

and turn out to be exactly the Einstein-Maxwell vacuum field equations. Thus, by another formalism, Einstein and Mayer rederived what Klein had obtained in his first paper on Kaluza's theory [185].

The authors' conclusion is:

"From the theory presented here, the equations for the gravitational and the electromagnetic fields follow effortlessly by a unifying method; however, up to now, [the theory] does not bring any understanding for the way corpuscles are built, nor for the facts comprised by quantum theory." ${ }^{170}$ ([107], p. 19)

After this paper Einstein wrote to Ehrenfest in a letter of 17 September 1931 that this theory "in my opinion definitively solves the problem in the macroscopic domain" ([241], p. 333). Also, in a lecture given on 14 October 1931 in the Physics Institute of the University of Wien, he still was proud of the 5-vector approach. In talking about the failed endeavours to reconcile classical field theory and quantum theory ("a cemetery of buried hopes") he is reported to have said:

"Since 1928 I also tried to find a bridge, yet left that road again. However, following an idea half of which came from myself and half from my collaborator, Prof. Dr. Mayer, a startlingly simple construction became successful. [...] According to my and Mayer's opinion, the fifth dimension will not show up. [...] according to which relationships between a hypothetical five-dimensional space and the four-dimensional can be obtained.

\footnotetext{
${ }^{169}$ The curly bracket was introduced in Equation (29).

170 "Die hier dargelegte Theorie liefert die Gleichungen des Gravitationsfeldes und des elektromagnetischen Feldes zwanglos auf einheitlichem Wege; dagegen liefert sie vorläufig kein Verständnis für den Bau der Korpuskeln sowie für die in der Quantentheorie zusammengefassten Tatsachen."
} 
In this way, we succeeded to recognise the gravitational and electromagnetic fields as a logical unity." 171 [96]

In his letter to Besso of 30 October 1931, Einstein seemed intrigued by the mathematics used in his paper with Mayer, but not enthusiastic about the physical content of this projective formulation of Kaluza's unitary field theory:

"The only result of our investigation is the unification of gravitation and electricity, whereby the equations for the latter are just Maxwell's equations for empty space. Hence, no physical progress is made, [if at all] at most only in the sense that one can see that Maxwell's equations are not just first approximations but appear on as good a rational foundation as the gravitational equations of empty space. Electrical and mass-density are non-existent; here, splendour ends; perhaps this already belongs to the quantum problem, which up to now is unattainable from the point of view of field [theory] (in the same way as relativity is from the point of view of quantum mechanics). The witty point is the introduction of 5 -vectors $a^{\sigma}$ in fourdimensional space, which are bound to space by a linear mechanism. Let $a^{s}$ be the 4 -vector belonging to $a^{\sigma}$; then such a relation $a^{s}=\gamma_{\sigma}^{s} a^{\sigma}$ obtains. In the theory equations are meaningful which hold independently of the special relationship generated by $\gamma_{\sigma}^{s}$. Infinitesimal transport of $a^{\sigma}$ in fourdimensional space is defined, likewise the corresponding 5-curvature from which spring the field equations." ${ }^{172}$ ([99], pp. 274-275)

In his report for the Macy-Foundation, which appeared in Science on the very same day in October 1931, Einstein had to be more optimistic:

"This theory does not yet contain the conclusions of the quantum theory. It furnishes, however, clues to a natural development, from which we may anticipate further developments in this direction. In any event, the results thus far obtained represent a definite advance in knowledge of the structure of physical space." ([94], p. 439)

Unfortunately, as in the case of his previous papers on Kaluza's theory, Einstein came in only second: Veblen had already worked on projective geometry and projective connections for a couple of years [374, 376, 375]. One year prior to Einstein's and Mayer's publication, with his student Hoffmann ${ }^{173}$, he had suggested an application to physics equivalent to the Kaluza-Klein

\footnotetext{
171 "Auch ich habe seit 1928 einen Ausgleich zu finden gesucht, diesen Weg aber wieder verlassen. Dagegen gelang eine verblüffend einfache Konstruktion auf Grund einer Idee, die zur Hälfte von mir, zur Hälfte von meinem Mitarbeiter Prof. Dr. Mayer stammt. [...] Nach meiner und Mayers Auffassung tritt die fünfte Dimension nicht in Erscheinung. [...] demzufolge man Beziehungen zwischen einem hypothetischen fünfdimensionalen Raum und dem vierdimensionalen aufstellen kann. Auf diese Weise gelang es, das Gravitations- und das elektromagnetische Feld als logische Einheit zu erfassen."

172 "Das Einzige, was in unserer Untersuchung herauskommt, ist die Vereinigung von Gravitation und Elektrizität, wobei die Gleichungen der letzteren genau die (relativistisch geschriebenen) Maxwell'schen des leeren Raumes sind Es ist also kein physikalischer Fortschritt dabei, höchstens nur insoweit als man eben sieht, dass die Maxwell'schen Gleichungen nicht nur erste Näherungen sind, sondern ebensogut rationell begründet erscheinen wie die Gravitationsgleichungen des leeren raumes. Elektrische und Massendichte gibt es hierbei nicht; da hört die Herrlichkeit auf; dies gehört wohl schon zum Quantenproblem, das bis jetzt vom Feldstandpunkt unerreichbar ist (ebensowenig wie die Relativität vom Standpunkt der Quantenmechanik aus). Der Witz liegt in der Einführung von Fünfervektoren $a^{\sigma}$ im vierdimensionalen Raum, die an den Raum durch einen linearen Mechanismus gebunden sind. $a^{s}$ sei der zu $a^{\sigma}$ gehörende Vierervektor, dann gibt es eine solche Beziehung $a^{\sigma}=\gamma_{\sigma}^{s} a^{\sigma}$. Sinnvoll sind dann in der Theorie solche Gleichungen, welche unabhängig von der durch $\gamma_{\sigma}^{s}$ geschaffenen besonderen Beziehung gelten. Infinitesimale Verschiebung von $\left(a^{\sigma}\right)$ im vierdim. Raum wird definiert, ebenso die dazu gehörige Fünferkrümmung und diese liefert dann die Feldgleichungen."

173 Banesh Hoffmann (1906-1986). Born in Richmond, England. Studied mathematics and theoretical physics at Oxford University and received his doctorate in 1929. Became an assistant at Princeton University and worked there with Einstein in 1932-1935. (His name supplied the "H" in the EIH paper.) From 1939 professor at Queens College in New York. His scientific interests were in relativity theory, tensor analysis, and quantum theory.
} 
theory [381, 163]. However, according to Pauli, Veblen and Hoffmann had spoiled the advantage of projective theory:

"But these authors choose a formulation that, due to an unnecessary specialisation of the coordinate system, prefers the fifth coordinate relative to the remaining [coordinates] in much the same way as this had happened in Kaluza-Klein theory by means of the cylinder condition [...]." ${ }^{174}$ ([249], p. 307)

By using the idea that an affine $(n+1)$-space can be represented by a projective $n$-space [413], Veblen and Hoffmann avoided the five dimensions of Kaluza: There is a one-to-one correspondence between the points of space-time and a certain congruence of curves in a five-dimensional space for which the fifth coordinate is the curves' parameter, while the coordinates of space-time are fixed. The five-dimensional space is just a mathematical device to represent the events (points) of space-time by these curves. Geometrically, the theory of Veblen and Hoffmann is more transparent and also more general than Einstein and Mayer's: It can house the additional scalar field inherent in Kaluza's original approach. Thus, Veblen and Hoffmann also gained the Klein-Gordon equation in curved space, i.e., an equation with the Ricci scalar $R$ appearing besides its mass term. Interestingly, the curvature term reads as $\frac{5}{27} R$ ([381], p. 821). In his note, Hoffmann generalised the formalism such as to include Dirac's equations (without gravitation), although some technical difficulties remained. Nevertheless, Hoffman remained optimistic:

"There is thus a possibility that the complete system will constitute an improved unification within the relativity theory of the gravitational, electromagnetic and quantum aspects of the field." ([163], p. 89)

In his book, Veblen emphasised

"[...] that our theory starts from a physical and geometrical point of view totally different from KALUZA's. In particular, we do not demand a relationship between electrical charge and a fifth coordinate; our theory is strictly four-dimensional." 175 [379]

Shortly after Einstein's and Mayer's paper had appeared, Schouten and van Dantzig also proved that the 5-vector formalism of this paper can be brought into a projective form [314].

In a second note, Einstein and Mayer extended the 5-vector-formalism to include Maxwell's equations with a non-vanishing current density [109]. Of the three basic assumptions of the previous paper, the second had to be given up. The expression in the middle of Equation (153) is replaced by

$$
\gamma_{k ; l}^{\iota}=A^{\iota} F_{k l}+\gamma^{\iota r} V_{r l k},
$$

where, again, $F_{k l}=-F_{l k}$, and the new $V_{r l k}=V_{r k l}$ are arbitrary tensors. The field equations were set up according to the method of the first paper; now the 5-curvature scalar was $P=$ $R-F_{k p} F^{k p}-V_{r q p} V^{r p q}$. It also turned out that $V^{r p q}=\epsilon^{l r p q} \phi_{l}$ with $\phi_{l}=\frac{\partial \phi}{\partial x^{l}}$, i.e., that the introduction of $V_{r p q}$ brought only one additional variable. The electric current density became $\sim V_{p r q} F^{r q}$.

In the last paragraph, the compatibility of the equations was proven, and at the end Cartan was acknowledged:

\footnotetext{
174 "Diese Autoren wählen aber eine Formulierung, die infolge unnötiger Spezialisierung des Koordinatensystems die fünfte Koordinate in ganz ähnlicher Weise vor den übrigen auszeichnet wie dies bei Kaluza-Klein durch die Zylinderbedingung geschehen war [...]."

175 “[...] dass unsere Theorie von ganz anderen physikalischen und geometrischen Gesichtspunkten als die KALUZAsche ausgeht. Insbesondere fordern wir kein Verhältnis zwischen elektrischer Ladung und einer fünften Koordinate; unsere Theorie ist vielmehr durchaus vierdimensional."
} 
"We note that Mr. Cartan, in a general and very illuminating investigation, has analysed more deeply the property of systems of differential equations that has been termed by us 'compatibility' in this paper and in previous papers." ${ }^{176}$ [37]

At about the same time as Einstein and Mayer wrote their second note, van Dantzig continued his work on projective geometry $[361,362,360]$. He used homogeneous coordinates $X^{\alpha}$, with $\alpha=1, \ldots, 5$, and the invariant $g_{\alpha \beta} X^{\alpha} X^{\beta}$, and introduced projectors and covariant differentiation (cf. Section 2.1.3). Together with him, Schouten wrote a series of papers on projective geometry as the basis of a unified field theory $[316,317,315,318]^{177}$, which, according to Pauli, combine

"all advantages of the formulations of Kaluza-Klein and Einstein-Mayer while avoiding all their disadvantages." ([249], p. 307)

Both the Einstein-Mayer theory and Veblen and Hoffmann's approach turned out to be subcases of the more general scheme of Schouten and van Dantzig intending

"to give a unification of general relativity not only with Maxwell's electromagnetic theory but also with Schrödinger's and Dirac's theory of material waves." ([318], p. 271)

In this paper ([318], p. 311, Figure 2), we find an early graphical representation of the parametrised set of all possible theories of a kind ${ }^{178}$. The formalism of Schouten and van Dantzig allows for taking the additional dimension to be timelike; in their physical applications the metric of spacetime is taken as a Lorentz metric; torsion is also included in their geometry.

Pauli, with his student J. Solomon ${ }^{179}$, generalised Klein, and Einstein and Mayer by allowing for an arbitrary signature in an investigation concerning "the form that take Dirac's equations in the unitary theory of Einstein and Mayer" 180 [253]. In a note added after proofreading, the authors showed that they had noted Schouten and Dantzig's papers [316, 317]. The authors pointed out that

"[...] even in the absence of gravitation we must pay attention to a difference between Dirac's equation in the theory of Einstein and Mayer, and Dirac's equation as it is written out, usually." ${ }^{181}$ ([253], p. 458)

The second order wave equation iterated from their form of Dirac's equation, besides the spin term contained a curvature term $-\frac{1}{4} R$, with the numerical factor different from Veblen's and Hoffmann's. In a sequel to this publication, Pauli and Solomon corrected an error:

"We examine from a general point of view the theory of spinors in a five-dimensional space. Then we discuss the form of the energy-momentum tensor and of the current vector in the theory of Einstein-Mayer.[...] Unfortunately, it turned out that the considerations of §in the first part are marred by a calculational error... This has made it necessary to introduce a new expression for the energy-momentum tensor and [...] likewise for the current vector [...]." ${ }^{182}$ ([254], p. 582)

\footnotetext{
176 "Wir bemerken, dass Herr Cartan in einer allgemeinen und überaus aufklärenden Untersuchung jene Eigenschaft von Differentialgleichungssystemen tiefer analysiert hat, welche von uns in dieser Arbeit und in früheren Arbeiten als 'Kompatibilität' bezeichnet wurde."

${ }^{177}$ van Dantzig ventured even into physics; he wrote a paper on Miller's repeat of Michelson's experiment but published it in a mathematics journal [359].

${ }^{178}$ A similar picture is already given in [315], p. 666, Figure 2

${ }^{179}$ J. Solomon came from Copenhagen to Zürich on a Rockerfeller grant.

180 "la forme que prennent les équations de Dirac dans la théorie unitaire d'Einstein et Mayer"

181 “[...] même en l'absence de gravitation nous devons nous attendre à une différence entre l'equation de Dirac dans la théorie d'Einstein et Mayer et l'équation de Dirac telle qu'elle est écrite habituellement."

182 "On y examine d'un point de vue général la théorie des spinors dans l'espace à cinq dimensions. On discute ensuite la forme du tenseur énergie-quantité de mouvement et du vecteur de courant dans la théorie d'Einstein et Mayer.[...] Malheureusement il s'est montré que les considérations du $\S 7$ de la première partie sont entachées d'une faute de calcul. . Ceci a rendu nécessaire l'introduction d'une nouvelle expression pour le tenseur énergie-quantité de mouvement et [...] également pour le vecteur de courant [...]."
} 
In the California Institute of Technology, Einstein's and Mayer's new mathematical technique found an attentive reader as well; A. D. Michal and his co-author generalised the Einstein-Mayer 5-vector-formalism:

"The geometry considered by Einstein and Mayer in their 'Unified field theory' leads to the consideration of an $n$-dimensional Riemannian space $V_{n}$ with a metric tensor $g_{i j}$, to each point of which is associated an $m$-dimensional linear vector space $V_{m},(m>n)$, for which vector spaces a general linear connection is defined. For the general case $(m-n \neq 1)$ we find that the calculation of the $m-n$ 'exceptional directions' is not unique, and that an additional postulate on the linear connection is necessary. Several of the new theorems give new results even for $n=4, m=5$, the Einstein-Mayer case." [228]

Michal had come from Cartan and Schouten's papers on group manifolds and the distant parallelisms defined on them [227]. H. P. Robertson found a new way of applying distant parallelism: He studied groups of motion admitted by such spaces, e.g., by Einstein's and Mayer's spherically symmetric exact solution [282] (cf. Section 6.4.3).

Cartan wrote a paper on the Einstein-Mayer theory as well ([39], an article published only posthumously) in which he showed that this could be interpreted as a five-dimensional flat geometry with torsion, in which space-time is embedded as a totally geodesic subspace.

\subsection{Distant parallelism}

The next geometry Einstein took as a fundament for unified field theory was a geometry with Riemannian metric, vanishing curvature, and non-vanishing torsion, named "absolute parallelism", "distant parallelism", "teleparallelism, or "Fernparallelismus". The contributions from the LeviCivita connection and from contorsion ${ }^{183}$ in the curvature tensor cancel. In place of the metric, tetrads are introduced as the basic variables. As in Euclidean space, in the new geometry these 4-beins can be parallely translated to retain the same fixed directions everywhere. Thus, again, a degree of absoluteness is re-introduced into geometry in contrast to Weyl's first attempt at unification which tried to soften the "rigidity" of Riemannian geometry.

The geometric concept of "fields of parallel vectors" had been introduced on the level of advanced textbooks by Eisenhart as early as 1925-1927 [119, 121] without use of the concept of a metric. In particular, the vanishing of the (affine) curvature tensor was given as a necessary and sufficient condition for the existence of $D$ linearly independent fields of parallel vectors in a $D$-dimensional affine space ([121], p. 19).

\subsubsection{Cartan and Einstein}

As concerns the geometry of "Fernparallelism", it is a special case of a space with Euclidean connection introduced by Cartan in 1922/23 [31, 30, 32]. Pais let Einstein "invent" and "discover" distant parallelism, and he states that Einstein "did not know that Cartan was already aware of this geometry" ([241], pp. 344-345). However, when Einstein published his contributions in June 1928 [84, 83], Cartan had to remind him that a paper of his introducing the concept of torsion had

"appeared at the moment at which you gave your talks at the Collège de France. I even remember having tried, at Hadamard's place, to give you the most simple example of a Riemannian space with Fernparallelismus by taking a sphere and by treating as parallels two vectors forming the same angle with the meridians going through their

183 a linear combination of torsion appearing in the connection besides the metric contribution (cf. Equation (43)). 
two origins: the corresponding geodesics are the rhumb lines." ${ }^{184}$ (letter of Cartan to Einstein on 8 May 1929; cf. [50], p. 4)

This remark refers to Einstein's visit in Paris in March/April 1922. Einstein had believed to have found the idea of distant parallelism by himself. In this regard, Pais may be correct. Every researcher knows how an idea, heard or read someplace, can subconsciously work for years and then surface all of a sudden as his or her own new idea without the slightest remembrance as to where it came from. It seems that this happened also to Einstein. It is quite understandable that he did not remember what had happened six years earlier; perhaps, he had not even fully followed then what Cartan wanted to explain to him. In any case, Einstein's motivation came from the wish to generalise Riemannian geometry such that the electromagnetic field could be geometrized:

"Therefore, the endeavour of the theoreticians is directed toward finding natural generalisations of, or supplements to, Riemannian geometry in the hope of reaching a logical building in which all physical field concepts are unified by one single viewpoint." 185 ([84], p. 217)

In an investigation concerning spaces with simply transitive continuous groups, Eisenhart already in 1925 had found the connection for a manifold with distant parallelism given 3 years later by Einstein [118]. He also had taken up Cartan's idea and, in 1926, produced a joint paper with Cartan on "Riemannian geometries admitting absolute parallelism" [40], and Cartan also had written about absolute parallelism in Riemannian spaces [33]. Einstein, of course, could not have been expected to react to these and other purely mathematical papers by Cartan and Schouten, focussed on group manifolds as spaces with torsion and vanishing curvature ([41, 34], pp. 50-54). No physical application had been envisaged by these two mathematicians.

Nevertheless, this story of distant parallelism raises the question of whether Einstein kept up on mathematical developments himself, or whether, at the least, he demanded of his assistants to read the mathematical literature. Against his familiarity with mathematical papers speaks the fact that he did not use the name "torsion" in his publications to be described in the following section. In the area of unified field theory including spinor theory, Einstein just loved to do the mathematics himself, irrespective of whether others had done it before - and done so even better (cf. Section 7.3).

Anyhow, in his response (Einstein to Cartan on 10 May 1929, [50], p. 10), Einstein admitted Cartan's priority and referred also to Eisenhart's book of 1927 and to Weitzenböck's paper [393]. He excused himself by Weitzenböck's likewise omittance of Cartan's papers among his 14 references. In his answer, Cartan found it curious that Weitzenböck was silent because

"[...] he indicates in his bibliography a note by Bortolotti in which he several times refers to my papers." ${ }^{186}$ (Cartan to Einstein on 15 May 1929; [50], p. 14)

The embarrassing situation was solved by Einstein's suggestion that he had submitted a comprehensive paper on the subject to Zeitschrift für Physik, and he invited Cartan to add his description of the historical record in another paper (Einstein to Cartan on 10 May 1929). After Cartan had sent his historical review to Einstein on 24 May 1929, the latter answered three months later:

\footnotetext{
184 "Parue au moment oú vous faisiez vos conférences au Collège de France; je me rappelle même avoir, chez M. Hadamard, essayé de vous donner l'exemple le plus simple d'un espace de Riemann avec Fernparallelismus en prenant une sphère et en regardand commes paralléles deux vecteurs faisant le même angle avec les méridiennes qui passent par leurs deux origines: les géodésiques correspondantes sont les loxodromies."

185 "Deshalb ist das Bestreben der Theoretiker darauf gerichtet, natürliche Verallgemeinerungen oder Ergänzungen der RIEMANNschen Geometrie aufzufinden, welche begriffsreicher sind als diese, in der Hoffnung, zu einem logischen Gebäude zu gelangen, das alle physikalischen Feldbegriffe unter einem einzigen Gesichtspunkte vereinigt."

186 "[...] il indique dans sa bibliographie une note de Bortolotti dans laquelle il se réfère plusieurs fois à mes travaux."
}

Living Reviews in Relativity

http: //www. livingreviews.org/lrr-2004-2 
"I am now writing up the work for the Mathematische Annalen and should like to add yours [...]. The publication should appear in the Mathematische Annalen because, at present, only the mathematical implications are explored and not their applications to physics." ${ }^{187}$ (letter of Einstein to Cartan on 25 August 1929 [50, 35, 89])

In his article, Cartan made it very clear that it was not Weitzenböck who had introduced the concept of distant parallelism, as valuable as his results were after the concept had become known. Also, he took Einstein's treatment of Fernparallelism as a special case of his more general considerations. Interestingly, he permitted himself to interpet the physical meaning of geometrical structures $^{188}$ :

"Let us say simply that mechanical phenomena are of a purely affine nature whereas electromagnetic phenomena are essentially metric; therefore it is rather natural to try to represent the electromagnetic potential by a not purely affine vector." ${ }^{189}$ ([35], p. 703)

Einstein explained:

"In particular, I learned from Mr. Weitzenböck and Mr. Cartan that the treatment of continua of the species which is of import here, is not really new.[...] In any case, what is most important in the paper, and new in any case, is the discovery of the simplest field laws that can be imposed on a Riemannian manifold with Fernparallelismus." 190 ([89], p. 685)

For Einstein, the attraction of his theory consisted

"For me, the great attraction of the theory presented here lies in its unity and in the allowed highly overdetermined field variables. I also could show that the field equations, in first approximation, lead to equations that correspond to the Newton-Poisson theory of gravitation and to Maxwell's theory. Nevertheless, I still am far from being able to claim that the derived equations have a physical meaning. The reason is that I could not derive the equations of motion for the corpuscles." ${ }^{191}$ ([89], p. 697)

The split, in first approximation, of the tetrad field $h_{a b}$ according to $h_{a b}=\eta_{a b}+\bar{h}_{a b}$ lead to homogeneous wave equations and divergence relations for both the symmetric and the antisymmetric part identified as metric and electromagnetic field tensors, respectively.

\subsubsection{How the word spread}

Einstein in 1929 really seemed to have believed that he was on a good track because, in this and the following year, he published at least 9 articles on distant parallelism and unified field theory

\footnotetext{
187 Mathematische Annalen was a journal edited by David Hilbert with co-editors O. Blumenthal and G. Hecke which physicists usually would not read. Einstein had been co-editor for the volumes 81 (1920) to 100 (1928); thus he had easy access. The editor of Zeitschrift für Physik was Karl Scheel, an experimental physicist.

${ }^{188}$ In view of van Dantzig's later papers showing that the vacuum Maxwell equations depend neither on the concept of metric nor of connection, Cartan's reasons underlying his remark are not obvious [367, 363, 364, 365, 366].

189 "Remarquons simplement qu'en principe les phénomènes mécaniques sont de nature purement affine, tandis que les phénomènes électromagnétiques sont de nature essentiellement métrique; il peut donc assez naturel de chercher à représenter le potentiel électromagnétique par un vecteur non purement affine."

190 "Insbesondere durch die Herren Weitzenböck und Cartan erfuhr ich, dass die Behandlung von Kontinua der hier in Betracht kommenden Gattung an sich nicht neu sei. [...] Was an der vorliegenden Abhandlung das Wichtigste und jedenfalls neu ist, das ist die Auffindung der einfachsten Feldgesetze, welche eine Riemannsche Mannigfaltigkeit mit Fernparallelismus unterworfen werden kann."

191 "in ihrer Einheitlichkeit und der hochgradigen (erlaubten) Überbestimmung der Feldvariablen. Auch habe ich zeigen können, dass die Feldgleichungen in erster Näherung auf Gleichungen führen, welche der Newton-Poissonschen Theorie der Gravitation und der Maxwellschen Theorie des elektromagnetischen Feldes entsprechen. Trotzdem bin ich noch weit davon entfernt, die physikalische Gültigkeit der abgeleiteten Gleichungen behaupten zu können. Der Grund liegt darin, dass mir die Ableitung von Bewegungsgesetzen für die Korpuskeln noch nicht gelungen ist."
} 
before switching off his interest. The press did its best to spread the word: On 2 February 1929, in its column News and Views, the respected British science journal Nature reported:

"For some time it has been rumoured that Prof. Einstein has been about to publish the results of a protracted investigation into the possibility of generalising the theory of relativity so as to include the phenomena of electromagnetism. It is now announced that he has submitted to the Prussian Academy of Sciences a short paper in which the laws of gravitation and of electromagnetism are expressed in a single statement."

Nature then went on to quote from an interview of Einstein of 26 January 1929 in a newspaper, the Daily Chronicle. According to the newspaper, among other statements Einstein made, in his wonderful language, was the following:

"Now, but only now, we know that the force which moves electrons in their ellipses about the nuclei of atoms is the same force which moves our earth in its annual course about the sun, and it is the same force which brings to us the rays of light and heat which make life possible upon this planet." [2]

Whether Einstein used this as a metaphorical language or, whether he at this time still believed that the system "nucleus and electrons" is dominated by the electromagnetic force, remains open.

The paper announced by Nature is Einstein's "Zur einheitlichen Feldtheorie", published by the Prussian Academy on 30 January 1929 [88]. A thousand copies of this paper had been sold within 3 days, so the presiding secretary of the Academy ordered the printing of a second thousand. Normally, only a hundred copies were printed ([183], Dokument Nr. 49, p. 136). On 4 February 1929, The Times (of London) published the translation of an article by Einstein, "written as an explanation of his thesis for readers who do not possess an expert knowledge of mathematics". This article then became reprinted in March by the British astronomy journal The Observatory [86]. In it, Einstein first gave a historical sketch leading up to the introduction of relativity theory, and then described the method that guided him to the new theory of distant parallelism. In fact, the only formulas appearing are the line elements for two-dimensional Riemannian and Euclidean space. At the end, by one figure, Einstein tried to convey to the reader what consequence a Euclidean geometry with torsion would have - without using that name. His closing sentences are ${ }^{192}$ :

"Which are the simplest and most natural conditions to which a continuum of this kind can be subjected? The answer to this question which I have attempted to give in a new paper yields unitary field laws for gravitation and electromagnetism." ([86], p. 118)

A few months later in that year, again in Nature, the mathematician H. T. H. Piaggio gave an exposition for the general reader of "Einstein's and other Unitary Field Theories". He was a bit more explicit than Einstein in his article for the educated general reader. However, he was careful to end it with a warning:

"Of course the ultimate test of the theory must be by experiment. It may succeed in predicting some interaction between gravitation and electromagnetism which can be confirmed by observation. On the other hand, it may be only a 'graph' and so outside the ken of the ordinary physicist." ([258], p. 879)

The use of the concept "graph" had its origin in Eddington's interpretation of his and other peoples' unified field theories to be only graphs of the world; the true geometry remained the Riemannian geometry underlying Einstein's general relativity.

\footnotetext{
${ }^{192}$ Einstein's policy was to permit only articles for the general reader to be printed in newspapers; he discouraged an English translation of his first teleparallelism paper of 1929 asked for by a publishing house (see [183], Documents Nr. 57 and 58, p. 141).
}

Living Reviews in Relativity

http: //www. livingreviews . org/lrr-2004-2 
Even the French-Belgian writer and poet Maurice Maeterlinck had heard of Einstein's latest achievement in the area of unified field theory. In his poetic presentation of the universe "La grande féerie" 193 we find his remark:

"Einstein, in his last publications comments to which are still to appear, again brings us mathematical formulae which are applicable to both gravitation and electricity, as if these two forces seemingly governing the universe were identical and subject to the same law. If this were true it would be impossible to calculate the consequences." 194 ([214], p. 68)

\subsubsection{Einstein's research papers}

We are dealing here with Einstein's, and Einstein and Mayer's joint papers on distant parallelism in the reports of the Berlin Academy and Mathematische Annalen, which were taken as the starting point by other researchers following suit with further calculations. Indeed, there was a lot of work to do, only in part because Einstein, from one paper to the next, had changed his field equations ${ }^{195}$.

In his first note [84], dynamics was absent; Einstein made geometrical considerations his main theme: Introduction of a local " $n$-bein-field" $h_{\hat{\imath}}^{k}$ at every point of a differentiable manifold and the related object $h_{k \hat{\imath}}$ defined as the collection of the "normed subdeterminants of the $h_{\hat{\imath}}^{k}$ " 196 such that $h_{i \hat{l}} h_{\hat{l}}^{k}=\delta_{i}^{k}$. As we have seen before, the components of the metric tensor are defined by

$$
g_{l m}=h_{l \hat{\jmath}} h_{m \hat{\jmath}},
$$

where summation over $\hat{\jmath}=1, \ldots, n$ is assumed ${ }^{197}$.

"Fernparallelism" now means that if the components referred to the local $n$-bein of a vector $A^{\hat{k}}=h_{l}^{\hat{k}} A^{l}$ at a point $p$, and of a vector $B^{\hat{k}}$ at a different point $q$ are the same, i.e., $A^{\hat{k}}=B^{\hat{k}}$, then the vectors are to be considered as "parallel". There is an underlying symmetry, called "rotational invariance" by Einstein: joint rotations of each $n$-bein by the same angle. All relations with a physical meaning must be "rotationally invariant". Of course, in space-time with a Lorentz metric, the 4-bein-transformations do form the proper Lorentz group.

If parallel transport of a tangent vector $A$ is defined as usual by $d A^{k}=-\Delta_{l m}{ }^{k} A^{l} d x^{m}$, then the connection components turn out to be

$$
\Delta_{l m}^{k}=h^{k \hat{\jmath}} \frac{\partial h_{l \hat{\jmath}}}{\partial x^{m}}
$$

An immediate consequence is that the covariant derivative of each bein-vector vanishes,

$$
h_{\hat{\jmath} ; l}^{k}:=h_{\hat{\jmath}, l}^{k}+\Delta_{s l}^{k} h_{\hat{\jmath}}^{s}=0
$$

by use of Equation (161). Also, the metric is covariantly constant

$$
g_{i k ; l}=0 .
$$

${ }^{193}$ German edition: "Geheimnisse des Weltalls" (secrets of the universe).

194 "Einstein wiederum bringt uns in seiner letzten Veröffentlichung, deren Kommentare noch ausstehen, mathematische Formeln, die gleichzeitig auf die Schwerkraft und die Elektrizität anwendbar sind, als wären diese beiden Kräfte, die das Weltall zu lenken scheinen, identisch und demselben Gesetz unterworfen. Wenn dem so wäre, würden die Folgen unberechenbar sein."

${ }^{195}$ The non-specialised reader will find this section rather technical.

196 "normed subdeterminants" means that the subdeterminants are divided by the determinant det $h_{\hat{\imath}}^{k}$. Thus, the inverse matrix must be calculated.

${ }^{197}$ Unlike in Einstein's notation, which used Greek indices for the coordinate indices and Latin ones for the beins, here bein-indices are noted by a hat-symbol. Consequently, Latin indices are to be moved by the metric $g_{i k}$, while bein-indices are raised and lowered by $\delta_{\hat{\imath} \hat{k}}$ or, for real coordinates, by the Minkowski metric. 
Neither fact is mentioned in Einstein's note. Also, no reference is given to Eisenhart's paper of 1925 [118], in which the connection (161) had been given (Equation (3.5) on p. 248 of [118]), as noted above, its metric-compatibility shown, and the vanishing of the curvature tensor concluded.

The (Riemannian) curvature tensor calculated from Equation (161) turns out to vanish. As Einstein noted, by $g_{i j}$ from Equation (160) also the usual Riemannian connection $\Gamma_{l m}^{k}(g)$ may be formed. Moreover, $Y_{l m}{ }^{k}:=\Gamma_{l m}^{k}(g)-\Delta_{l m}^{k}$ is a tensor that could be used for building invariants. In principle, distant parallelism is a particular bi-connection theory. The connection $\Gamma_{l m}^{k}(g)$ does not play a role in the following (cf., however, de Donder ${ }^{198}$ 's paper [48]).

From Equation (161), obviously the torsion tensor $S_{l m}^{k}=\frac{1}{2}\left(\Delta_{l m}^{k}-\Delta_{m l}^{k}\right) \neq 0$ follows (cf. Equation (21)). Einstein denoted it by $\Lambda_{l m}^{k}$ and, in comparison with the curvature tensor, considered it as the "formally simplest" tensor of the theory for building invariants by help of the linear form $\Lambda_{l j}{ }^{j} d x^{l}$ and of the scalars $g^{i j} \Lambda_{i m}^{l} \Lambda_{j l}{ }^{m}$ and $g_{i j} g^{l r} g^{m s} \Lambda_{l m}^{i} \Lambda_{r s}^{j}$. He indicated how a Lagrangian could be built and the 16 field equations for the field variables $h_{l j}$ obtained.

At the end of the note Einstein compared his new approach to Weyl's and Riemann's:

- WEYL: Comparison at a distance neither of lengths nor of directions;

- RIEMANN: Comparison at a distance of lengths but not of directions;

- Present theory: Comparison at a distance of both lengths and directions.

In his second note [83], Einstein departed from the Lagrangian $\mathcal{L}=h g^{i j} \Lambda_{i m}{ }^{l} \Lambda_{j l}{ }^{m}$, i.e., a scalar density corresponding to the first scalar invariant of his previous note ${ }^{199}$. He introduced $\phi_{k}:=\Lambda_{k l}^{l}$, and took the case $\phi=0$ to describe a "purely gravitational field". However, as he added in a footnote, pure gravitation could have been characterised by $\frac{\partial \phi_{i}}{\partial x^{k}}-\frac{\partial \phi_{k}}{\partial x^{i}}$ as well. In his first paper on distant parallelism, Einstein did not use the names "electrical potential" or "electrical field". He then showed that in a first-order approximation starting from $h_{i \hat{\jmath}}=\delta_{i \hat{\jmath}}+k_{i \hat{\jmath}}+\ldots$, both the Einstein vacuum field equations and Maxwell's equations are surfacing. To do so he replaced $h_{i \hat{\jmath}}$ by $g_{i j}=\delta_{i j}+2 k_{(i j)}$ and introduced $\phi_{k}:=\frac{1}{2}\left(\frac{\partial k_{k j}}{\partial x^{j}}-\frac{\partial k_{j j}}{\partial x^{k}}\right)$. Einstein concluded that

"The separation of the gravitational and the electromagnetic field appears artificial in this theory. [...] Furthermore, it is remarkable that, according to this theory, the electromagnetic field does not enter the field equations quadratically." ${ }^{200}$ ([83], p. 6)

In a postscript, Einstein noted that he could have obtained similar results by using the second scalar invariant of his previous note, and that there was a certain indeterminacy as to the choice of the Lagrangian.

This shows clearly that the ambiguity in the choice of a Lagrangian had bothered Einstein. Thus, in his third note, he looked for a more reassuring way of deriving field equations [88]. He left aside the Hamiltonian principle and started from identities for the torsion tensor, following from the vanishing of the curvature tensor ${ }^{201}$. He thus arrived at the identity given by Equation (29),

\footnotetext{
198 Théophile Ernest de Donder (1872-1957). Born in Brussels. Studied mathematics and physics at the University of Brussels and received his doctorate in 1899. Professor of mathematical physics at the Université libre de Bruxelles from 1911 to 1942. Member of the Royal Belgian Academy. Research on variational calculus, general relativity, electromagnetism, thermodynamics, and wave mechanics.

${ }^{199} h:=\operatorname{det} h_{l \hat{m}}$ corresponds to $\sqrt{-\operatorname{det} g_{i j}}$. In his next note [88] Einstein also switched his notation of the 4-leg to Weitzenböck's ${ }^{s} h_{k}$, where the index to the left of $h$ counts the number of legs.

200 "die Trennung des Gravitationsfeldes und des elektromagnetischen Feldes erscheint aber nach dieser Theorie als künstlich. [...] Bemerkenswert ist ferner, dass nach dieser Theorie das elektrische Feld nicht quadratisch in die Feldgleichungen eingeht."

${ }^{201}$ There are some misprints in the formulae of $\S 1$ of Einstein's paper [88]. He writes the tensor density $V$ with an upper coordinate and two lower bein-indices, i.e., as $\hat{V}_{\hat{k} \hat{l}}{ }^{j}$. Einstein also introduced a new covariant derivative for which he used the symbol $A_{/ k}$. We call it $\nabla *_{l}$; it is given through Equation (16), i.e., by $\nabla_{k} X^{i}=\nabla^{*}-\Delta_{k r}^{r} \hat{X}^{i}$.
} 
i.e., (Einstein's equation (3), p. 5; his convention is $\Lambda_{\hat{k} \hat{l}}^{\hat{\imath}}:=2 S_{\hat{k} \hat{l}}{ }^{\imath}$ )

$$
0=2 \nabla_{\{\hat{\jmath}} S_{\hat{k} \hat{l}\}}^{\hat{\imath}}+4 S_{\hat{m}\{\hat{\jmath}}^{\hat{\imath}} S_{\hat{k} \hat{l}\}}^{\hat{m}} .
$$

By defining $\phi_{\hat{k}}:=\Lambda_{\hat{k} \hat{l}}^{\hat{l}}=2 S_{\hat{k} \hat{l}}^{\hat{l}}$, and contracting equation (164), Einstein obtained another identity $^{202}$ :

$$
\nabla *_{j} \hat{V}_{\hat{k} \hat{l}}^{j}=0
$$

where the covariant divergence refers to the connection components $\Delta_{\hat{m} \hat{l}}^{\hat{k}}$, and the tensor density $\hat{V}_{\hat{k} \hat{l}}^{\hat{\jmath}}$ is given by

$$
\hat{V}_{\hat{k} \hat{l}}^{\hat{\jmath}}:=2 h\left(S_{\hat{k} \hat{l}}^{\hat{\jmath}}+\phi_{[\hat{l}} \delta_{\hat{k}]}^{\hat{\jmath}}\right) .
$$

For the proof, he used the formula for the covariant vector density given in Equation (16), which, for the divergence, reduces to $\stackrel{+}{\nabla}_{i} X^{i}=\frac{\partial \hat{X}^{i}}{\partial x^{i}}-2 S_{j} \hat{X}^{j}$.

The second identity used by Einstein follows with the help of Equation (27) for vanishing curvature (Einstein's equation (5), p. 5):

$$
\stackrel{+}{\nabla}_{[j} \stackrel{+}{\nabla}_{k]} \quad \hat{A}^{j k}=-S_{j k}^{r} \stackrel{+}{\nabla}_{r} \hat{A}^{j k} .
$$

As we have seen in Section 2, if he had read it, Einstein could have taken these identities from Schouten's book of 1924 [300]. is

By replacing $\hat{A}^{j k}$ by $\hat{V}^{\hat{k} \hat{l} j}$ and using Equation (165), the final form of the second identity now

$$
\nabla *_{j}\left(\nabla_{\hat{l}} \hat{V}^{\hat{k} \hat{l} j}-2 \hat{V}^{\hat{k} l r} S_{l r}{ }^{j}\right)=0 .
$$

Einstein first wrote down a preliminary set of field equations from which, in first approximation, both the gravitational vacuum field equations (in the limit $\epsilon=0$, cf. below) and Maxwell's equations follow:

$$
\nabla *_{j} \hat{U}_{\hat{k} \hat{l}}^{j}=0, \quad \nabla *_{r} \nabla *_{l} \hat{V}_{\hat{k}}^{l r}=0 .
$$

Here,

$$
\hat{U}_{\hat{k} \hat{l}}^{\hat{\jmath}}:=\hat{V}_{\hat{k} \hat{l}}^{\hat{\jmath}}-2 \epsilon h \phi_{[\hat{l}} \delta_{\hat{k}]}^{\hat{\jmath}}
$$

replaces $\hat{V}_{k l}^{j}$ such that the necessary number of equations is obtained. With this first approximation as a hint, Einstein, after some manipulations, postulated the 20 exact field equations:

$$
\nabla *_{\hat{l}} \hat{V}^{\hat{k} \hat{l} r}-2 \hat{V}^{\hat{k} r s} S_{s r}{ }^{l}=0, \quad \nabla *_{j}\left[h p h i^{[k ; j]}\right]=0,
$$

among which 8 identities hold.

Einstein seems to have sensed that the average reader might be able to follow his path to the postulated field equations only with difficulty. Therefore, in a postscript, he tried to clear up his motivation:

"The field equations suggested in this paper may be characterised with regard to other such possible ones in the following way. By staying close to the identity (167), it has been accomplished that not only 16 , but 20 independent equations can be imposed on the 16 quantities $h_{i}^{\hat{k}}$. By 'independent' we understand that none of these equations can

${ }^{202}$ The abbreviation $A_{\| k}$ is used only in place of $\stackrel{+}{\nabla}_{k}$. If summation over indices is to be performed, it is irrelevant whether bein-indices or coordinate indices are written. 
be derived from the remaining ones, even if there exist 8 identical (differential) relations among them." 203 ([88], p. 8)

He still was not entirely sure that the theory was physically acceptable:

"A deeper investigation of the consequences of the field equations (170) will have to show whether the Riemannian metric, together with distant parallelism, really gives an adequate representation of the physical qualities of space." 204

In his second paper of 1929, the fourth in the series in the Berlin Academy, Einstein returned to the Hamiltonian principle because his collaborators Lanczos and Müntz ${ }^{205}$ had doubted the validity of the field equations of his previous publication [88] on grounds of their unproven compatibility. In the meantime, however, he had found a Lagrangian such that the compatibility-problem disappeared. He restricted the many constructive possibilities for $\mathcal{L}=\mathcal{L}\left(h_{i}^{\hat{k}}, \partial_{l} h_{i}^{\hat{k}}\right)$ by asking for a Lagrangian containing torsion at most quadratically. His Lagrangian is a particular linear combination of the three possible scalar densities, as follows:

(1) $\hat{H}=\frac{1}{2} \hat{\jmath}_{1}+\frac{1}{4} \hat{\jmath}_{2}-\hat{\jmath}_{3}$,

(2) $\hat{H}^{*}=\frac{1}{2} \hat{\jmath}_{1}-\frac{1}{4} \hat{\jmath}_{2}$,

(3) $\hat{H}^{* *}=\hat{\jmath}_{3}$,

with $\hat{\jmath}_{1}:=h S_{k l}{ }^{m} S_{m}^{k}{ }^{l}, \hat{\jmath}_{2}:=h S_{k l}{ }^{m} S^{k l}{ }_{m}$, and $\hat{\jmath}_{3}:=h S_{j} S^{j}$. If $\epsilon_{1}, \epsilon_{2}$ are small parameters, then his final Lagrangian is $\mathcal{L}=\hat{H}+\epsilon_{1} \hat{H}^{*}+\epsilon_{2} \hat{H}^{* *}$. In order to prove that Maxwell's equations follow from his Lagrangian, Einstein had to perform the limit $\sigma:=\frac{\epsilon_{1}}{\epsilon_{2}} \rightarrow 0$ in an expression termed $\hat{G}^{* i k}$, which he assumed to depend homogeneously and quadratically on a linear combination of torsion $^{206}$.

In a Festschrift for his former teacher and colleague in Zürich, A. Stodola, Einstein summed up what he had reached ${ }^{207}$. He exchanged the definition of the invariants named $\hat{\jmath}_{2}$, and $\hat{\jmath}_{3}$, and stated that a choice of $A=-B, C=0$ in the Lagrangian $\hat{\jmath}=A_{\hat{\jmath}_{1}}+B_{\hat{\jmath}_{2}}+C_{\hat{\jmath}_{3}}$ would give field equations

\footnotetext{
203 "Die in dieser Arbeit vorgeschlagenen Feldgleichungen sind formal gegenüber sonst denkbaren so zu kennzeichnen. Es ist durch Anlehnung an die Identität (167) erreicht worden, dass die 16 Grössen $h_{i}^{\hat{k}}$ nicht nur 16 , sondern 20 selbständigen Differentialgleichungen unterworfen werden können. Unter 'selbständig' ist dabei verstanden, dass keine dieser Gleichungen aus den übrigen gefolgert werden kann, wenn auch zwischen ihnen 8 identische (Differentiations-) Relationen bestehen."

204 "Eine tiefere Untersuchung der Konsequenzen der Feldgleichungen (170) wird zu zeigen haben, ob die RIEMANN-Metrik in Verbindung mit dem Fernparallelismus wirklich eine adäquate Auffassung der physikalischen Qualitäten des Raumes liefert."

205 As to the person of H. Müntz, it is not obvious whether he can be identified with Dr. Ch. Müntz, a possibility following from a paper of Ch. H. Müntz, presented to the Göttingen Academy by D. Hilbert in 1917. If it is the same person, then H. Müntz seems to have been a mathematics teacher, first at the Odenwaldschule in Heppenheim a.d. B. from 1918 to 1922(?), then, possibly for a short time in Göttingen (Friedländerweg 61), and from 1924 on in BerlinNikolassee, Herkrathstr. 5. I conclude this from the membership lists of the Deutsche Mathematikervereinigung, which Müntz entered in 1913, and which gives a Berlin address since July 1924 and lists him as "Prof." in Berlin, in 1931. At the time, experienced teachers at Gymnasium could carry the title of professor. In the Einstein archive, 26 letters of Einstein to Müntz from the years 1927-1931 exist. The addresses show that Müntz went to Stockhom via Tallin. In fact, Pais [241] writes that Müntz became a professor of mathematics at the University of Leningrad but had to leave the Soviet Union in 1938 for Sweden. In fact, a document of 1931 states: "Prof. Hermann Mueninz, der einer der engeren wissenschaftlichen Mitarbeiter Albert Einsteins ist und gegenwärtig ein Lehramt für höhere Mathematik an der Leningrader Universität bekleidet [...]" ([183], Dokument 144, p. 222). Sauer ([289], p.11) reports the life span of Müntz to have been 1884-1956.

${ }^{206}$ The variational derivatives of $\hat{H}, \hat{H}^{*}$, and $\hat{H}^{* *}$ are named $\hat{G}^{i k}, \hat{G}^{* i k}$, and $\hat{G}^{* * i k}$, respectively, by Einstein.

${ }^{207}$ Einstein's contribution seems to have been submitted towards the end of 1928 (cf. [289], p. 21).
} 
"[...] that coincide in first approximation with the known laws for the gravitational and electromagnetic field [...]"208

with the proviso that the specialisation of the constants $A, B, C$ must be made only after the variation of the Lagrangian, not before. Also, together with Müntz, he had shown that for an uncharged mass point the Schwarzschild solution again obtained [87].

Einstein's next publication was the note preceding Cartan's paper in Mathematische Annalen [89]. He presented it as an introduction suited for anyone who knew general relativity. It is here that he first mentioned Equations (162) and (163). Most importantly, he gave a new set of field equations not derived from a variational principle; they are ${ }^{209}$.

$$
\begin{aligned}
& G^{i k}:=S_{.}^{i l{ }^{k}}{ }_{\| l}-S^{i m n}{ }^{i m} S_{n m}{ }^{k}=0, \\
& F_{i k}:=\quad S_{i k}{ }^{l}{ }^{l} l=0,
\end{aligned}
$$

where $S^{i k l}=g^{i m} g^{k n} S_{m n}{ }^{l}$. There exist 4 identities among the $16+6$ field equations

$$
G_{\| l}^{i l}-F_{\| l}^{i l}+S_{.}^{i m n} F_{n m}=0 .
$$

As Cartan remarked, Equation (172) expresses conservation of torsion under parallel transport:

"In fact, in the new theory of Mr. Einstein, it is natural to call a universe homogeneous if the torsion vectors that are associated to two parallel surface elements are parallel themselves; this means that parallel transport conserves torsion." ${ }^{210}$ ([35], p. 703)

From Equation (173) with the help of Equation (171), (172), Einstein wrote down two more identities. One of them he had obtained from Cartan:

"But I am very grateful to you for the identity

$$
G^{i k}{ }_{\| i}-S_{l m}^{k} G^{l m}=0
$$

which, astonishingly, had escaped me. [...] In a new presentation in the Sitzungsberichten, I used this identity while taking the liberty of pointing to you as its source." 211 (letter of Einstein to Cartan from 18 December 1929, Document X of [50], p. 72)

In order to show that his field equations were compatible he counted the number of equations, identities, and field quantities (in $n$-dimensional space) to find, in the end, $n^{2}+n$ equations for the same number of variables. To do so, he had to introduce an additional variable $\psi$ via $F_{k}=\phi_{k}-\frac{\partial \log \psi}{\partial x^{k}}$. Here, $F_{k}$ is introduced by $F_{i k}=\partial_{k} F_{i}-\partial_{i} F_{k}=\partial_{k} \phi_{i}-\partial_{i} \phi_{k}$. Einstein then showed that $\partial_{l} F_{i k}+\partial_{k} F_{l i}+\partial_{i} F_{k l}=0$.

208 “...] welche mit den bekannten Gesetzen des Gravitationsfeldes und des elektromagnetischen Feldes in erster Näherung übereinstimmen [...]"

${ }^{209}$ The quantity $G^{i k}$ defined here must be distinguished from the Einstein tensor in Section 2.2 denoted by the same symbol.

210 "En effet, avec la nouvelle théorie de M. Einstein, il est naturel d'appeler homogène un univers où les vecteurs de torsion associés à deux éléments de surface sont paralléles eux-mêmes; c'est à dire où le transport paralléle conserve la torsion."

211 "Sehr dankbar bin ich Ihnen aber für die Identität

$$
G^{i k}{ }_{\| i}-S_{l m}{ }^{k} G^{l m}=0,
$$

die mir merkwürdigerweise entgangen war. [...] In einer neuen Darstellung in den Sitzungsberichten habe ich von dieser Identität Gebrauch gemacht, indem ich mir erlaubte, auf Sie als Quelle aufmerksam zu machen." 
The changes in his approach Einstein continuously made, must have been hard on those who tried to follow him in their scientific work. One of them, Zaycoff ${ }^{212}$, tried to make the best out of them:

"Recently, A. Einstein ([89]), following investigations by E. Cartan ([35]), has considerably modified his teleparallelism theory such that former shortcomings (connected only to the physical identifications) vanish by themselves." ${ }^{213}$ ([433], p. 410)

In November 1929, Einstein gave two lectures at the Institute Henri Poincaré in Paris which had been opened one year earlier in order to strengthen theoretical physics in France ([14], pp. 263272). They were published in 1930 as the first article in the new journal of this institute [92]. On 23 pages he clearly and leisurely outlined his theory of distant parallelism and the progress he had made. As to references given, first Cartan's name is mentioned in the text:

"It is not for the first time that such spaces are envisaged. From a purely mathematical point of view they were studied previously. M. Cartan was so amiable as to write a note for the Mathematische Annalen exposing the various phases in the formal development of these concepts." 214 ([92], p. 4)

Note that Einstein does not say that it was Cartan who first "envisaged" these spaces before. Later in the paper, he comes closer to the point:

"This type of space had been envisaged before me by mathematicians, notably by WEITZENBÖCK, EISENHART et CARTAN [...]." ${ }^{215}$ [92]

Again, he held back in his support of Cartan's priority claim.

Some of the material in the paper overlaps with results from other publications [85, 90, 93]. The counting of independent variables, field equations, and identities is repeated from Einstein's paper in Mathematische Annalen [89]. For $n=4$, there were 20 field equations $\left(G^{i k}=0, F^{l}=0\right)$ for $16+1$ variables $h_{\hat{\imath}}^{k}$ and $\psi$, four of which were arbitrary (coordinate choice). Hence 7 identities should exist, four of which Einstein had found previously. He now presented a derivation of the remaining three identities by a calculation of two pages' length. The field equations are the same as in [89]; the proof of their compatibility takes up, in a slightly modified form, the one communicated by Einstein to Cartan in a letter of 18 December 1929 ([92], p. 20). It is reproduced also in [90].

Interestingly, right after Einstein's article in the institute's journal, a paper of C. G. Darwin, "On the wave theory of matter", is printed, and, in the same first volume, a report of Max Born on "Some problems in Quantum Mechanics." Thus, French readers were kept up-to-date on progress made by both parties - whether they worked on classical field theory or quantum theory [45, 21].

A. Proca, who had attended Einstein's lectures, gave an exposition of them in a journal of his native Romania. He was quite enthusiastic about Einstein's new theory:

"A great step forward has been made in the pursuit of this total synthesis of phenomena which is, right or wrong, the ideal of physicists. [...] the splendid effort brought about

\footnotetext{
212 Gawrilow Raschko Zaycoff (1901-1982). Born in Burgas, Bulgaria. Studied at the Universities of Sofia, Göttingen, and Berlin from 1922 to 1928. From 1928 assistant in the Physics Institute of the University of Sofia; 1931-1933 teacher at a Gymnasium in Sofia. From 1935 on mathematical statistician at the Institute for Economic Research of Sofia University. From 1961-1972 Professor at the Physics Institute of the Bulgarian Academy of Science 213 "Neuerdings hat A. Einstein gestützt auf Untersuchungen von E. Cartan, seine Fernparallelismustheorie wesentlich modifiziert, so dass die früheren Nachteile (es handelt sich nur um die physikalischen Identifikationen) derselben von selbst hinfällig werden."

214 "Ce n'est pas la première fois qu'on envisage de tels espaces. Du point de vue purement mathématique ils ont déjà été étudié auparavant. M. CARTAN a eu l'amabilité de rédiger, pour les Mathematische Annalen, une note exposant les diverses phases du développement formel de ces conceptions."

215 "Ce type d'espace à été envisagé, avant moi, par des mathématiciens, notamment par WEITZENBÖCK, EISENHART et CARTAN [...]."
} 
by Einstein permits us to hope that the last theoretical difficulties will be vanquished, and that we soon will compare the consequences of the theory with [our] experience, the great stepping stone of all creations of the mind." 216 [260, 261]

Einstein's next paper in the Berlin Academy, in which he reverts to his original notation $h_{l}^{\hat{k}}$, consisted of a brief critical summary of the formalism used in his previous papers, and the announcement of a serious mistake in his first note in 1930, which made invalid the derivation of the field equations for the electromagnetic field ([90], p. 18). The mistake was the assumption on the kind of dependence on torsion of the quantity $\hat{G}^{*} i k$, which was mentioned above. Also, Einstein now found it better "to keep the concept of divergence, defined by contraction of the extension of a tensor" and not use the covariant derivative $\nabla *_{l}$ introduced by him in his third paper in the Berlin Academy [88].

Then Einstein presented the same field equations as in his paper in Annalen der Mathematik, which he demanded to be

(1) covariant,

(2) of second order, and

(3) linear in the second derivatives of the field variable $h_{i}^{k}$.

While these demands had been sufficient to uniquely lead to the gravitational field equations (with cosmological constant) of general relativity, in the teleparallelism theory a great deal of ambiguity remained. Sixteen field equations were needed which, due to covariance, induced four identities.

"Therefore equations must be postulated among which identical relations are holding. The higher the number of equations (and consequently also the number of identities among them), the more precise and stronger than mere determinism is the content; accordingly, the theory is the more valuable, if it is also consistent with the empirical facts." 217 ([90], p. 21)

He then gave a proof of the compatibility of his field equations:

"The proof of the compatibility, as given in my paper in the Mathematische Annalen, has been somewhat simplified due to a communication which I owe to a letter of Mr. CARTAN (cf. $\S 3,[16]) . " 218$

The reader had to make out for himself what Cartan's contribution really was.

In linear approximation, i.e., for $h_{i k}=\delta_{i k}+\bar{h}_{i k}$, Einstein obtained d'Alembert's equation for both the symmetric and the antisymmetric part of $\bar{h}_{i k}$, identified with the gravitational and the electromagnetic field, respectively.

Einstein's next note of one and a half pages contained a mathematical result within teleparallelism theory: From any tensor with an antisymmetric pair of indices a vector with vanishing divergence can be derived [93].

\footnotetext{
216 "Un grand pas en avant a été fait dans la poursuite de cette synthèse totale des phénomènes qui est, à tort ou à raison, l'idéal des physiciens. [...] le splendide effort fourni par Einstein nous permet d'espérer que les dernières difficultés théoriques seront vaincues et que nous pourrons bientôt comparer les conséquences de la théorie à l'expérience, cette pierre d'achoppement de toutes les créations de l'esprit."

217 "Es müssen also Gleichungen aufgestellt werden, zwischen denen identische Relationen bestehen. Je höher die Zahl der Gleichungen ist (und folglich auch der zwischen ihnen bestehenden Identitäten), desto bestimmtere, über die Forderung des blossen Determinismus hinausgehende Aussagen macht die Theorie; desto wertvoller ist also die Theorie, falls sie mit den Erfahrungstatsachen verträglich ist."

218 "Der Kompatibilitätsbeweis ist auf Grund einer brieflichen Mitteilung, welche ich Herrn CARTAN verdanke (vgl. §3, [16]), gegenüber der in den Mathematischen Annalen gegebene Darstellung etwas vereinfacht."
} 
In order to test the field equations by exhibiting an exact solution, a simple case would be to take a spherically symmetric, asymptotically (Minkowskian) 4-bein. This is what Einstein and Mayer did, except with the additional assumption of space-reflection symmetry [106]. Then the 4-bein contains three arbitrary functions of one parameter $s$ :

$$
h_{\hat{\iota}}^{\alpha}=\lambda(s) \delta_{\hat{\iota}}^{\alpha}, \quad h_{\hat{4}}^{\alpha}=\tau(s) x_{\alpha}, \quad h_{\hat{\iota}}^{4}=0, \quad h_{\hat{4}}^{\hat{4}}=u(s),
$$

where $\alpha, \hat{\imath}=1,2,3$. As an exact solution of the field equations (171, 172), Einstein and Mayer obtained $\left.\lambda=\tau=e s^{-3}\left(1-e^{2} / s^{4}\right)^{-\frac{1}{4}}\right)$ and $u=1+m \int d s s^{-2}\left(1-e^{2} / s^{4}\right)^{-\frac{1}{4}}$. The constants $e$ and $m$ were interpreted as electric charge and "ponderomotive mass," respectively. A further exact solution for uncharged point particles was also derived; it is static and corresponds to "two or more unconnected electrically neutral masses which can stay at rest at arbitrary distances". Einstein and Mayer do not take this physically unacceptable situation as an argument against the theory, because the equations of motion for such singularities could not be derived from the field equations as in general relativity. Again, the continuing wish to describe elementary particles by singularity-free exact solutions is stressed.

Possibly, W. F. G. Swan of the Bartol Research Foundation in Swarthmore had this paper in mind when he, in April 1930, in a brief description of Einstein's latest publications, told the readers of Science:

"It now appears that Einstein has succeeded in working out the consequences of his general law of gravity and electromagnetism for two special cases just as Newton succeeded in working out the consequences of his law for several special cases. [...] It is hoped that the present solutions obtained by Einstein, or if not these, then others which may later evolve, will suggest some experiments by which the theory may be tested." ([339], p. 391)

Two days before the paper by Einstein and Mayer became published by the Berlin Academy, Einstein wrote to his friend Solovine:

"My field theory is progressing well. Cartan has already worked with it. I myself work with a mathematician (S. Mayer ${ }^{219}$ from Vienna), a marvelous chap [...]." ${ }^{220}$ ([98], p. 56)

The mentioning of Cartan resulted from the intensive correspondence of both scientists between December 1929 and February 1930: About a dozen letters were exchanged which, sometimes, contained long calculations [50] (cf. Section 6.4.6). In an address given at the University of Nottingham, England, on 6 June 1930, Einstein also must have commented on the exact solutions found and on his program concerning the elementary particles. A report of this address stated about Einstein's program:

"The problem is nearly solved; and to the first approximations he gets laws of gravitation and electro-magnetics. He does not, however, regard this as sufficient, though those laws may come out. He still wants to have the motions of ordinary particles to come out quite naturally. [The program] has been solved for what he calls the 'quasistatical motions', but he also wants to derive elements of matter (electrons and protons) out of the metric structure of space." ([91], p. 610)

With his "assistant" Walther Mayer, Einstein then embarked on a very technical, systematic study of compatible field equations for distant parallelism [108]. In addition to the assumptions (1), (2), (3) for allowable field equations given above, further restrictions were made:

\footnotetext{
${ }^{219}$ Note that Einstein used the initial "S" in place of the correct "W" (Walther)

220 "Meine Feldtheorie macht gute Fortschritte. Cartan hat schon darin gearbeitet. Ich selbst arbeite mit einem Mathematiker (S. Mayer aus Wien), einem prächtigen Kerl [...]."
} 
(4) the field equations must contain the first derivatives of the field variable $h_{i}^{\hat{k}}$ only quadratically;

(5) the identities for the left hand sides $G^{i k}$ of the field equations must be linear in $G^{i k}$ and contain only their first derivatives;

(6) torsion must occur only linearly in $G^{i k}$.

For the field equations, the following ansatz was made:

$$
0=G^{i k}=p S_{. \cdot{ }^{i l ~ k l}}^{k}+q S_{.}^{k l} \stackrel{i}{\| l}+a_{1} \phi_{.}^{i \| k}+a_{2} \phi_{.}^{k \| i}+a_{3} g_{.}^{i k} \phi_{\| l}^{l}+R^{i k}
$$

where $R^{i k}$ is a collection of terms quadratic in torsion $S$, and $p, q, a_{1}, a_{2}, a_{3}$ are constants. They must be determined in such a way that the "divergence-identity"

$$
G_{; i}^{i k}+G_{; i}^{k i}+G^{l m}\left(c_{1} S_{l m}^{k}+c_{2} S_{l}^{\cdot m}+c_{3} S_{m}^{\cdot l}{ }_{k}\right)+c_{4} G^{k l} \phi_{l}+c_{5} G^{l k} \phi_{l}+c_{6} G_{l}^{\cdot{ }^{l}} \phi_{.}^{k}+B G_{l \| \cdot}^{l \cdot k}=0
$$

is satisfied. Here, 8 new constants $A, B, c_{r}$ with $r=1, \ldots, 6$ to be fixed in the process also appear. After inserting Equation (175) into Equation (176), Einstein and Mayer reduced the problem to the determination of 10 constants by 20 algebraic equations by a lengthy calculation. In the end, four different types of compatible field equations for the teleparallelism theory remained:

"Two of these are (non-trivial) generalisations of the original gravitational field equations, one of them being known already as a consequence of the Hamiltonian principle.

The remaining two types are denoted in the paper by [...]."221

With no further restraining principles at hand, this ambiguity in the choice of field equations must have convinced Einstein that the theory of distant parallelism could no longer be upheld as a good candidate for the unified field theory he was looking for, irrespective of the possible physical content $^{222}$. Once again, he dropped the subject and moved on to the next. While aboard a ship back to Europe from the United States, Einstein, on 21 March 1932, wrote to Cartan:

"[...] In any case, I have now completely given up the method of distant parallelism. It seems that this structure has nothing to do with the true character of space [...]." ([50], p. 209)

What Cartan might have felt, after investing the forty odd pages of his calculations printed in Debever's book, is unknown. However, the correspondence on the subject came to an end in May 1930 with a last letter by Cartan. ${ }^{223}$.

\subsubsection{Reactions I: Mostly critical}

About half a year after Einstein's two papers on distant parallelism of 1928 had appeared, Reichenbach $^{224}$, who always tended to defend Einstein against criticism, classified the new theory [268] according to the lines set out in his book [267] as "having already its precisely fixed logical position

\footnotetext{
221 "Hiervon sind zwei (nichttriviale) Verallgemeinerungen der ursprünglichen Feldgleichungen der Gravitation, von denen eine als aus dem Hamiltonschen Prinzip hervorgehend bereits bekannt ist [...]. Die beiden übrigen sind in der Arbeit durch [...] bezeichnet."

${ }^{222}$ This view is supported by an (as yet unpublished) detailed investigation of Einstein's theory of distant parallelism by $T$. Sauer which I received 6 month after after having submitted this review [289]. Sauer uses unpublished correspondence in the Einstein Papers Archive, and points to "some historical and systematic similarities between the Fernparallelismus episode and the Entwurf theory, i.e., the precurser theory of general relativity theory pursued by Einstein in the years 1912-1915."

${ }^{223}$ An evaluation of this correspondence has been given in [15].

224 Hans Reichenbach (1891-1953). Philosopher of science, neo-positivist. Professor in Berlin, Istanbul, and Los Angeles. Wrote books on the foundations of relativity theory, probability, and quantum mechanics.
} 
in the edifice of Weyl-Eddington geometry" ([267], p. 683). He mentioned as a possible generalization an idea of Einstein's, in which the operation of parallel transport might be taken as integrable not with regard to length but with regard to direction: "a generalisation which already has been conceived by Einstein as I learned from him" ([267], p. 687) ${ }^{225}$.

As concerns parallelism at a distance, Reichenbach was not enthusiastic about Einstein's new approach:

"[...] it is the aim of Einstein's new theory to find such an entanglement between gravitation and electricity that it splits into the separate equations of the existing theory only in first approximation; in higher approximation, however, a mutual influence of both fields is brought in, which, possibly, leads to an understanding of questions unanswered up to now as [is the case] for the quantum riddle. But this aim seems to be in reach only if a direct physical interpretation of the operation of transport, even of the immediate field quantities, is given up. From the geometrical point of view, such a path [of approach] must seem very unsatisfactory; its justifications will only be reached if the mentioned link does encompass more physical facts than have been brought into it for building it up." ${ }^{226}$ ([267], p. 689)

A first reaction from a competing colleague came from Eddington, who, on 23 February 1929, gave a cautious but distinct review of Einstein's first three publications on distant parallelism [84, 83, 88] in Nature. After having explained the theory and having pointed out the differences to his own affine unified field theory of 1921, he confessed:

"For my own part I cannot readily give up the affine picture, where gravitational and electric quantities supplement one another as belonging respectively to the symmetrical and antisymmetrical features of world measurement; it is difficult to imagine a neater kind of dovetailing. Perhaps one who believes that Weyl's theory and its affine generalisation afford considerable enlightenment, may be excused for doubting whether the new theory offers sufficient inducement to make an exchange." [62]

Weyl was the next unhappy colleague; in connection with the redefinition of his gauge idea he remarked (in April/May 1929):

"[...] my approach is radically different, because I reject distant parallelism and keep to Einstein's general relativity. [...] Various reasons hold me back from believing in parallelism at a distance. First, my mathematical intuition a priori resists to accept such an artificial geometry; I have difficulties to understand the might who has frozen into rigid togetherness the local frames in different events in their twisted positions. Two weighty physical arguments join in [...] only by this loosening [of the relationship between the local frames] the existing gauge-invariance becomes intelligible. Second, the possibility to rotate the frames independently, in the different events, [...] is equivalent to the symmetry of the energy-momentum tensor, or to the validity of the conservation law for angular momentum." ${ }^{227}$ ([407], pp. 330-332.)

\footnotetext{
${ }^{225}$ In fact, this generalisation was to be worked out soon by V. Bargmann in 1930 [5] (cf. below).

226 “[... es ist das Ziel der neuen Theorie Einsteins, eine derartige Verkettung von Gravitation und Elektrizität zu finden, dass sie nur in erster Näherung in die getrennten Gleichungen der bisherigen Theorie zerspaltet, während sie in höherer Näherung einen gegenseitigen Einfluss der beiden Felder lehrt, der möglicherweise zum Verständnis bisher ungelöster Fragen, wie der Quantenrätsel, führt. Aber dieses Ziel scheint nur erreichbar zu sein unter Verzicht auf eine unmittelbare physikalische Interpretation der Verschiebungsoperation, ja sogar der eigentlichen Feldgrössen selbst. Vom geometrischen Standpunkt muss deshalb ein solcher Weg sehr unbefriedigend erscheinen; seine Rechtfertigung wird allein dadurch gegeben werden können, dass er durch die genannte Verkettung mehr physikalische Tatsachen umschliesst, als zu seiner Aufstellung in ihn hineingelegt wurden."

227 “...] unterscheidet sich mein Ansatz in radikaler Weise dadurch, dass ich den Fernparallelismus ablehne und
} 
As usual, Pauli was less than enthusiastic; he expressed his discontent in a letter to Hermann Weyl of 26 August 1929:

"First let me emphasize that side of the matter about which I fully agree with you: Your approach for incorporating gravitation into Dirac's theory of the spinning electron [...] I am as adverse with regard to Fernparallelismus as you are [...] (And here I must do justice to your work in physics. When you made your theory with $g_{i k}^{\prime}=\lambda g_{i k}$ this was pure mathematics and unphysical; Einstein rightly criticised and scolded you. Now the hour of revenge has come for you, now Einstein has made the blunder of distant parallelism which is nothing but mathematics unrelated to physics, now you may scold [him].)" 228 ([251], pp. 518-519)

Another confession of Pauli's went to Paul Ehrenfest:

"By the way, I now no longer believe in one syllable of teleparallelism; Einstein seems to have been abandoned by the dear Lord." 229 (Pauli to Ehrenfest 29 September $1929 ;[251]$, p. 524)

Pauli's remark shows the importance of ideology in this field: As long as no empirical basis exists, beliefs, hopes, expectations, and rationally guided guesses abound. Pauli's letter to Weyl from 1 July 1929 used non-standard language (in terms of science):

"I share completely your skeptical position with regard to Einstein's 4-bein geometry.

During the Easter holidays I have visited Einstein in Berlin and found his opinion on modern quantum theory reactionary." ${ }^{230}$ ([251], p. 506)

While the wealth of empirical data supporting Heisenberg's and Schrödinger's quantum theory would have justified the use of a word like "uninformed" or even "not up to date" for the description of Einstein's position, use of "reactionary" meant a definite devaluation.

Einstein had sent a further exposition of his new theory to the Mathematische Annalen in August 1928. When he received its proof sheets from Einstein, Pauli had no reservations to criticise him directly and bluntly:

"I thank you so much for letting be sent to me your new paper from the Mathematische

Annalen [89], which gives such a comfortable and beautiful review of the mathematical properties of a continuum with Riemannian metric and distant parallelism [...]. Unlike

an Einsteins klassischer Relativitätstheorie der Gravitation festhalte. [...] An den Fernparallelismus vemag ich aus mehreren Gründen nicht zu glauben. Erstens sträubt sich mein mathematisches Gefühl a priori dagegen, eine so künstliche Geometrie zu akzeptieren; es fällt mir schwer, die Macht zu begreifen, welche die lokalen Achsenkreuze in den verschiedenen Weltpunkten in ihrer verdrehten Lage zu starrer Gebundenheit aneinander hat einfrieren lassen. Es kommen [...] zwei gewichtige physikalische Gründe hinzu. [...] nur durch diese Lockerung [des Zusammenhangs zwischen den lokalen Achsenkreuzen] wird die tatsächlich bestehende Eichinvarianz verständlich. Und zweitens ist die Möglichkeit, die Achsenkreuze an verschiedenen Stellen unabhängig voneinander zu drehen [...] gleichbedeutend mit der Sy m metrie des Energi eimpulstensors oder mit der Gültigkeit des Erhaltungssatzes für das Impulsmoment."

228 "Zuerst will ich diejenige Seite der Sache hervorheben, bei der ich voll und ganz mit Ihnen übereinstimme: Ihr Ansatz zur Einordnung der Gravitation in die Diracsche Theorie des Spinelektrons. [...] Ich bin nämlich dem Fernparallelismus ebenso feindlich gesinnt wie Sie, [...]. (Und hier muss ich Ihrer Tätigkeit in der Physik Gerechtigkeit widerfahren lassen. Als Sie früher die Theorie mit $g_{i k}^{\prime}=\lambda g_{i k}$ machten, war dies reine Mathematik und unphysikalisch, Einstein konnte mit Recht kritisieren und schimpfen. Nun ist die Stunde der Rache für Sie gekommen; jetzt hat Einstein den Bock des Fernparallelismus geschossen, der auch nur reine Mathematik ist und nichts mit Physik zu tun hat, und Sie können schimpfen!)"

229 "Jetzt glaube ich übrigens vom Fernparallelismus keine Silbe mehr, den Einstein scheint der liebe Gott jetzt völlig verlassen zu haben."

230 "Ihre Skepsis bezüglich der Einsteinschen 4-Beingeometrie teile ich vollständig. In den Osterferien habe ich Einstein in Berlin besucht und fand seine Einstellung zur modernen Quantenphysik reaktionär." 
what I told you in spring, from the point of view of quantum theory, now an argument in favour of distant parallelism can no longer be put forward [...]. It just remains [...] to congratulate you (or should I rather say condole you?) that you have passed over to the mathematicians. Also, I am not so naive as to believe that you would change your opinion because of whatever criticism. But I would bet with you that, at the latest after one year, you will have given up the entire distant parallelism in the same way as you have given up the affine theory earlier. And, I do not wish to provoke you to contradict me by continuing this letter, because I do not want to delay the approach of this natural end of the theory of distant parallelism." ${ }^{231}$ (letter to Einstein of 19 December 1929; [251], 526-527)

Einstein answered on 24 December 1929:

"Your letter is quite amusing, but your statement seems rather superficial to me. Only someone who is certain of seeing through the unity of natural forces in the right way ought to write in this way. Before the mathematical consequences have not been thought through properly, is not at all justified to make a negative judgement. [...] That the system of equations established by myself forms a consequential relationship with the space structure taken, you would probably accept by a deeper study - more so because, in the meantime, the proof of the compatibility of the equations could be simplified." ${ }^{232}$ ([251], p. 582)

Before he had written to Einstein, Pauli, with lesser reservations, complained vis-a-vis Jordan:

"Einstein is said to have poured out, at the Berlin colloquium, horrible nonsense about new parallelism at a distance. The mere fact that his equations are not in the least similar to Maxwell's theory is employed by him as an argument that they are somehow related to quantum theory. With such rubbish he may impress only American journalists, not even American physicists, not to speak of European physicists." ${ }^{233}$ (letter of 30 November 1929, [251], p. 525)

Of course, Pauli's spells of rudeness are well known; in this particular case they might have been induced by Einstein's unfounded hopes for eventually replacing the Schrödinger-Heisenberg-Dirac quantum mechanics by one of his unified field theories.

\footnotetext{
231 "Ich danke Ihnen vielmals dafür, dass Sie die Korrekturen Ihrer neuen Arbeit aus den mathematischen Annalen [89] an mich senden liessen, die eine so bequeme und schöne Übersicht über die mathematischen Eigenschaften eines Kontinuums mit Riemann-Metrik und Fernparallelismus enthält. [...] Entgegen dem, was ich im Frühjahr zu Ihnen sagte, lässt sich vom Standpunkt der Quantentheorie nunmehr kein Argument zu Gunsten des Fernparallelismus mehr vorbringen. [...] Es bleibt [...] nur übrig, Ihnen zu gratulieren (oder soll ich lieber sagen: zu kondolieren?), dass Sie zu den reinen Mathematikern übergegangen sind. Ich bin auch nicht so naiv, dass ich glauben würde, Sie würden auf Grund irgendeiner Kritik durch Andere Ihre Meinung ändern. Aber ich würde jede Wette mit Ihnen eingehen, dass Sie spätestens nach einem Jahr den ganzen Fernparallelismus aufgegeben haben werden, so wie Sie früher die Affintheorie aufgegeben haben. Und ich will Sie nicht durch Fortsetzung dieses Briefes noch weiter zum Widerspruch reizen, um das Herannahen dieses natürlichen Endes der Fernparallelismustheorie nicht zu verzögern."

232 "Ihr Brief ist recht amüsant, aber Ihre Stellungnahme scheint mir doch etwas oberflächlich. So dürfte nur einer schreiben, der sicher ist, die Einheit der Naturkräfte vom richtigen Standpunkt aus zu überblicken. [...] Bevor die mathematischen Konsequenzen richtig durchgedacht sind, ist es keineswegs gerechtfertigt, darüber wegwerfend zu urteilen. [...] Dass das von mir aufgestellte Gleichungssystem zu der zugrundegelegten Raumstruktur in einer zwangsläufigen Beziehung steht, würden Sie bei tieferem Studium bestimmt einsehn, zumal der Kompatibilitätsbeweis der Gleichungen sich unterdessen noch hat vereinfachen lassen."

233 "Einstein soll im Berliner Kolloquium schrecklichen Quatsch über neuen Fernparallelismus verzapft haben! Die blosse Tatsache, dass seine Gleichungen nicht die geringste Ähnlichkeit mit der Maxwellschen Theorie haben, will er als Argument dafür hinstellen, dass sie etwas mit Quantentheorie zu tun haben. Mit einem solchen Kohl kann man nur amerikanischen Journalisten imponieren, nicht einmal amerikanischen Physikern, geschweige denn europäischen Physikern."
}

Living Reviews in Relativity

http: //www . livingreviews .org//rr-2004-2 
The question of the compatibility of the field equations played a very important role because Einstein hoped to gain, eventually, the quantum laws from the extra equations (cf. his extended correspondence on the subject with Cartan ([50] and Section 6.4.6).

That Pauli had been right (except for the time span envisaged by him) was expressly admitted by Einstein when he had given up his unified field theory based on distant parallelism in 1931 (see letter of Einstein to Pauli on 22 January 1932; cf. [241], p. 347).

Born's voice was the lonely approving one (Born to Einstein on 23 September 1929) ${ }^{234}$ :

"Your report on progress in the theory of Fernparallelism did interest me very much, particularly because the new field equations are of unique simplicity. Until now, I had been uncomfortable with the fact that, aside from the tremendously simple and transparent geometry, the field theory did look so very involved" ${ }^{235}$ ([154], p. 307)

Born, however, was not yet a player in unified field theory, and it turned out that Einstein's theory of distant parallelism became as involved as the previous ones.

Einstein's collaborator Lanczos even wrote a review article about distant parallelism with the title "The new field theory of Einstein" [201]. In it, Lanczos cautiously offers some criticism after having made enough bows before Einstein:

"To be critical with regard to the creation of a man who has long since obtained a place in eternity does not suit us and is far from us. Not as a criticism but only as an impression do we point out why the new field theory does not house the same degree of conviction, nor the amount of inner consistency and suggestive necessity in which the former theory excelled.[...] The metric is a sufficient basis for the construction of geometry, and perhaps the idea of complementing RIEMANNian geometry by distant parallelism would not occur if there were the wish to implant something new into RIEMANNian geometry in order to geometrically interpret electromagnetism." ${ }^{236}$ ([201], p. 126)

When Pauli reviewed this review, he started with the scathing remark

"It is indeed a courageous deed of the editors to accept an essay on a new field theory of Einstein for the 'Results in the Exact Sciences' [literal translation of the journal's title]. His never-ending gift for invention, his persistent energy in the pursuit of a fixed aim in recent years surprise us with, on the average, one such theory per year. Psychologically interesting is that the author normally considers his actual theory for a while as the 'definite solution'. Hence, [...] one could cry out: 'Einstein's new field theory is dead. Long live Einstein's new field theory!'”237 ([248], p. 186)

\footnotetext{
${ }^{234}$ This letter printed in Grüning's book is not contained in the Einstein-Born correspondence edited by Born [103]. 235 "Dein Bericht über die Fortschritte der Fernparallelismus-Theorie hat mich sehr interessiert und besonders, dass die neuen Feldgleichungen von einzigartiger Einfachheit sind. Bisher hat mich nämlich immer an der Sache gestört, dass neben der so ungeheuer einfachen und durchsichtigen Geometrie die Feldtheorie so äusserst verwickelt aussah."

236 "Kritik zu üben an der Schöpfung eines Mannes, der längst der Ewigkeit verschrieben ist, kommt uns nicht zu und liegt uns auch fern. Nicht als Kritik, lediglich als Eindruck sei darauf hingewiesen, weshalb der neuen Feldtheorie nicht jene Überzeugungskraft inne zu wohnen scheint, nicht jene innere Geschlossenheit und suggestive Notwendigkeit, die die frühere Theorie ausgezeichnet hat. [...] Die Metrik ist eine hinreichende Basis zum Aufbau der Geometrie und man würde wahrscheinlich nicht auf den Gedanken kommen, die RIEMANNsche Geometrie durch den Fernparallelismus zu ergänzen, wenn man nicht den Wunsch hätte, etwas Neues in die RIEMANNsche Geometrie hineinzukonstruieren, um den Elektromagnetismus geometrisch zu interpretieren."

237 "Es ist schon eine kühne Tat der Redaktion, ein Referat über eine neue Feldtheorie EINSTEINs unter die Ergebnisse der exakten Naturwissenschaften aufzunehmen. Beschert uns doch seine nie versagende Erfindungsgabe sowie seine hartnäckige Energie beim Verfolgen eines bestimmten Zieles in letzter Zeit durchschnittlich etwa eine solche Theorie pro Jahr - wobei es psychologisch interessant ist, dass die jeweilige Theorie vom Autor gewöhnlich eine Zeitlang als 'definitive' Lösung betrachtet wird. So könnte man [...] ausrufen 'Die neue Feldtheorie Einsteins ist tot. Es lebe die neue Feldtheorie Einsteins!'”
} 
For the remainder, Pauli engaged in a discussion with the philosophical background of Lanczos and criticised his support for Mie's theory of matter of 1913 according to which

"the atomism of electricity and matter, fully separated from the existence of the quantum of action, is to be reduced to the properties of (singularity-free) eigen-solutions of still-to-be-found nonlinear differential equations for the field variables." 238

Thus, Pauli lightly pushed aside as untenable one of Einstein's repeated motivations and hoped-for tests for his unified field theories.

Lanczos, being dissatisfied with Einstein's distant parallelism, then tried to explain "electromagnetism as a natural property of Riemannian geometry" by starting from the Lagrangian quadratic in the components of the Ricci tensor: $\mathcal{L}=R_{i k} R_{l m} g^{i l} g^{k m}+C\left(R_{i k} g^{i k}\right)^{2}$ with an arbitrary constant $C$. He varied $g_{i k}$ and $R_{i k}$ independently [202]. (For Lanczos see J. Stachel's essay "Lanczos' early contributions to relativity and his relation to Einstein" in [330], pp. 499-518.)

\subsubsection{Reactions II: Further research on distant parallelism}

The first reactions to Einstein's papers came quickly. On 29 October 1928, de Donder suggested a generalisation by using two metric tensors, a space-time metric $g_{i k}$, and a bein-metric $g_{i k}^{\star}$, connected to the 4-bein components $h_{l \hat{\jmath}}$ by

$$
g_{l m}=g_{\star}^{\hat{\jmath} \hat{k}} h_{l \hat{\jmath}} h_{m \hat{k}} .
$$

In place of Einstein's connection (161), defined through the 4-bein only, he took:

$$
\Delta_{l m}^{k}=\frac{1}{2} h^{k \hat{\jmath}}\left(h_{l \hat{\jmath} \cdot m}-h_{m \hat{\jmath} \cdot l}\right),
$$

where the dot-symbol denotes covariant derivation by help of the Levi-Civita connection derived from $g_{i k}^{\star}$. If the Minkowski metric is used as a bein metric $g^{\star}$, then the dot derivative reduces to partial derivation, and Einstein's original connection is obtained [48].

Another application of Einstein's new theory came from Eugen Wigner in Berlin whose paper showing that the tetrads in distant-parallelism-theory permitted a generally covariant formulation of "Diracs equation for the spinning electron", was received by Zeitschrift für Physik on 29 December 1928 [419]. He did point out that "up to now, grave difficulties stood in the way of a general relativistic generalisation of Dirac's theory" and referred to a paper of Tetrode [344]. Tetrode, about a week after Einstein's first paper on distant parallelism had appeared on 14 June 1928, had given just such a generally relativistic formulation of Dirac's equation through coordinate dependent Gamma-matrices ${ }^{239}$; he also wrote down a (symmetric) energy-momentum tensor for the Dirac field and the conservation laws. However, he had kept the metric $g_{i k}$ introduced into the formalism by

$$
\gamma_{i} \gamma_{k}+\gamma_{k} \gamma_{i}=2 g_{i k}
$$

to be conformally flat. For the matrix-valued 4 -vector $\gamma^{i}$ he prescribed the condition of vanishing divergence. Wigner did not fully accept Tetrode's derivations because there, implicitly and erroneously, it had been assumed that the two-dimensional representation of the Lorentz group (2-spinors) could be extended to a representation of the affine group. Wigner stated that such difficulties would disappear if Einstein's teleparallelism theory were used. Nevertheless, nowhere did

\footnotetext{
238 "soll der Atomismus von Elektrizität und Materie ganz losgelöst von der Existenz des Wirkungsquantums, auf die Eigenschaften von (singularitätenfreien) Eigenlösungen noch aufzufindender nichtlinearer Differentialgleichungen der Feldgrössen zurückgeführt werden."

${ }^{239}$ The paper extended a previous one within the framework of special relativity [345].
} 
he claim that the Dirac equation could only be formulated covariantly with the help of Einstein's new theory.

Zaycoff of the Physics Institute of the University in Sofia also followed Einstein's work closely. Half a year after Einstein's first two notes on distant parallelism had appeared [84, 83], i.e., shortly before Christmas 1928, Zaycoff sent off his first paper on the subject, whose arrival in Berlin was acknowledged only after the holidays on 13 January 1929 [429]. In it he described the mathematical formalism of distant parallelism theory, gave the identity (42), and calculated the new curvature scalar in terms of the Ricci scalar and of torsion. He then took a more general Lagrangian than Einstein and obtained the variational derivatives in linear and, in a simple example, also in second approximation. In his presentation, he used both the teleparallel and the Levi-Civita connections. His second and third papers came quickly after Einstein's third note of January 1929 [88], and thus had to take into account that Einstein had dropped derivation of the field equations from a variational principle. In his second paper, Zaycoff followed Einstein's method and gave a somewhat simpler derivation of the field equations. An exact, complicated wave equation followed:

$$
D_{l} D^{l} S_{k}-F_{k j} S^{j}-X_{k}=0
$$

where $X_{k}:=\frac{1}{2} D_{k}\left(V^{l m n} S_{l n m}\right)+S_{l k m} D_{r} S^{l r m}-\frac{1}{2} S_{k} V^{l m n} S_{l n m}+V^{l m n} S_{m n}^{r} S_{l k r}+S_{r k m} S^{r} S^{m}$ with torsion $S_{m n}{ }^{r}$ and the torsion vector $S_{k}$, and the covariant derivative $D_{l}:=\nabla_{l}-S_{l}$, $\nabla_{l}$ being the teleparallel connection (161). In linear approximation, the Einstein vacuum and the vacuum Maxwell equations are obtained, supplemented by the homogeneous wave equation for a vector field [431]. In his third note, Zaycoff criticised Einstein "for not having shown, in his most recent publication, whether his constraints on the world metric be permissible." He then derived additional exact compatibility conditions for Einstein's field equations to hold; according to him, their effect would show up only in second approximation [430]. In his fourth publication Zaycoff came back to Einstein's Hamiltonian principle and rederived for himself Einstein's results. He also defended Einstein against critical remarks by Eddington [62] and Schouten [304], although Schouten, in his paper, had mentioned neither Einstein nor his teleparallelism theory, but only gave a geometrical interpretation of the torsion vector in a geometry with semi-symmetric connection. Zaycoff praised Einstein's teleparallelism theory in words reminding me of the creation of the world as described in Genesis:

"We may say that A. Einstein built a plane world which is no longer waste like the Euclidean space-time-world of H. Minkowski, but, on the contrary, contains in it all that we usually call physical reality." 240 ([428], p. 724)

A conference on theoretical physics at the Ukrainian Physical-Technical Institute in Charkow in May 1929, brought together many German and Russian physicists. Unified field theory, quantum mechanics, and the new quantum field theory were all discussed. Einstein's former calculational assistant Grommer, now on his own in Minsk, in a brief contribution stressed Einstein's path for getting an overdetermined system of differential equations: Vary with regard to the 16 beinquantities but consider only the 10 metrical components as relevant. He claimed that Einstein had used only the antisymmetric part of the tensor $P_{l m}^{k}:=\Gamma(g)_{l m}{ }^{k}-\Delta_{l m}{ }^{k}$, where both $\Gamma(g)_{l m}{ }^{k}$ and $\Delta_{l m}{ }^{k}$ were mentioned above (in Einstein's first note) although Einstein never used $\Gamma(g)_{l m}{ }^{k}$. According to Grommer the anti-symmetry of $P$ is needed, because its contraction leads to the electromagnetic 4-potential and because the symmetric part can be expressed by the antisymmetric part and the metrical tensor. He also played the true voice of his (former) master by repeating Einstein's program of deriving the equations of motion from the overdetermined system:

240 "Man kann etwa sagen, dass A. Einstein eine ebene Welt aufgebaut hat, welche nicht mehr öde ist wie die euklidische Raum-Zeit-Welt von H. Minkowski, sondern im Gegenteil alles in sich enthält, was wir als physikalische Wirklichkeit zu bezeichnen pflegen." 
"If the law of motion of elementary particles could be derived from the overdetermined field equation, one could imagine that this law of motion permit only discrete orbits, in the sense of quantum theory." 241 ([153], p. 646)

Levi-Civita also had sent a paper on distant parallelism to Einstein, who had it appear in the reports of the Berlin Academy [207]. Levi-Civita introduced a set of four congruences of curves that intersect each other at right angles, called their tangents $\lambda_{\hat{\imath}}^{k}$ and used Equation (160) in the form:

$$
\delta_{l m}=h_{l \hat{\jmath}} h_{m \hat{\jmath}}
$$

He also employed the Ricci rotation coefficients defined by $\gamma_{\hat{\imath} \hat{k} \hat{l}}=\nabla_{\sigma} h_{\hat{\imath}}^{\beta} h_{\hat{k} \beta} h_{\hat{l}}^{\sigma}$, where the hatted indices are "bein"-indices; the Greek letters denote coordinates. They obey

$$
\gamma_{\hat{\imath} \hat{k} \hat{l}}+\gamma_{\hat{k} \hat{\imath} \hat{l}}=0
$$

The electromagnetic field tensor $F_{i k}$ was entered via

$$
F_{i k} \lambda_{\hat{l}}^{i} \lambda_{\hat{m}}^{k}=\lambda_{\hat{s}}^{r} \frac{\partial}{\partial x^{r}} \gamma_{\hat{l} \hat{m}}^{\hat{s}}
$$

Levi-Civita chose as his field equations the Einstein-Maxwell equations projected on a rigidly fixed "world-lattice" of 4-beins. He used the time until the printing was done to give a short preview of his paper in Nature [206]. About a month before Levi-Civita's paper was issued by the Berlin Academy, Fock and Ivanenko [135] had had the same idea and compared Einstein's notation and the one used by Levi-Civita in his monograph on the absolute differential calculus [205]:

"Einstein's new gravitational theory is intimately linked to the known theory of the orthogonal congruences of curves due to Ricci. In order to ease a comparison between both theories, we may bring together here the notations of $\mathrm{Ricci}$ and $\mathrm{Levi}-$ C i vi t a [...] with those of Einstein." 242

A little after the publication of Levi-Civita's papers, Heinrich Mandel embarked on an application of Kaluza's five-dimensional approach to Einstein's theory of distant parallelism [218]. Einstein had sent him the corrected proof sheets of his fourth paper [85]. The basic idea was to consider the points of $M_{4}$ as equivalent to the ensemble of congruences with tangent vector $X_{5}^{i}$ in $M_{5}$ (with cylindricity condition) ${ }^{243}$. The space-time interval is defined as the distance of two lines of the congruence on $M_{5}: d \tau^{2}=\left(\gamma_{i l}-X_{5 i} X_{5 l}\right)\left(\delta_{k}^{l}-X_{5}^{l} X_{5 k}\right) d x^{i} d x^{k}$. Mandel did not identify the torsion vector with the electromagnetic 4-potential, but introduced the covariant derivative $\triangle_{k} A^{i}:=\frac{\partial A^{i}}{\partial x^{k}}+\left\{\begin{array}{l}i \\ k j\end{array}\right\} A^{j}+\frac{e}{c} X_{5 k} \mathcal{M}_{l}^{i} A^{l}$, where the tensor $\mathcal{M}$ is skew-symmetric. We may look at this paper also as a forerunner of some sort to the Einstein-Mayer 5-vector formalism (cf. Section 6.3.2).

Before Einstein dropped the subject of distant parallelism, many more papers were written by a baker's dozen of physicists. Some were more interested in the geometrical foundations, in exact solutions to the field equations, or in the variational principle.

One of those hunting for exact solutions was G. C. McVittie who referred to Einstein's paper [88]:

\footnotetext{
241 "Würde man das Bewegungsgesetz der Elementarteilchen aus den überbestimmten Feldgleichungen ableiten können, so kann man sich denken, dass dieses Bewegungsgesetz nur diskrete Bahnen, im Sinne der Quantentheorie, gestatten wird."

242 "Die neue Einsteinsche Gravitationstheorie steht mit der bekannten, von Ricci herrührenden Theorie der orthogonalen Kurvenkongruenzen in engem Zusammenhang. Um den Vergleich der beiden Theorien zu erleichtern, mögen hier die Bezeichnungen von R i c i und Levi-Civita, [...] mit denjenigen von Einstein zusammengestellt werden."

${ }^{243}$ Mandel's $X_{5}^{i}$ corresponds to a 5 -bein vector $h_{\hat{5}}^{i}$.
} 
"[...] we test whether the new equations proposed by Einstein are satisfied. It is shown

that the new equations are satisfied to the first order but not exactly."

He then goes on to find a rigourous solution and obtains the metric $d s^{2}=e^{a x_{1}} d x_{4}^{2}-e^{-2 a x_{1}} d x_{1}^{2}-$ $e^{-a x_{1}} d x_{2}^{2}-e^{-a x_{1}} d x_{3}^{2}$ and the 4-potential $\phi_{4}=\frac{1}{2 \sqrt{\pi}} e^{\frac{1}{2} a x_{1}}$ [225]. He also wrote a paper on exact axially symmetric solutions of Einstein's teleparallelism theory [226].

Tamm and Leontowich treated the field equations given in Einstein's fourth paper on distant parallelism [85]. They found that these field equations did not have a spherically symmetric solution corresponding to a charged point particle at rest ${ }^{244}$. The corresponding solution for the uncharged particle was the same as in general relativity, i.e., Schwarzschild's solution. Tamm and Leontowitch therefore guessed that a charged point particle at rest would lead to an axially-symmetric solution and pointed to the spin for support of this hypothesis [342, 342].

Wiener $^{245}$ and Vallarta ${ }^{246}$ were after particular exact solutions of Einstein's field equations in the teleparallelism theory. By referring to Einstein's first two papers concerning distant parallelism, they set out to show that the

"[...] electromagnetic field is incompatible in the new Einstein theory with the assumption of static spherical symmetry and symmetry of the past and the future. [...] the new Einstein theory lacks at present all experimental confirmation."

In footnote 4 , they added:

"Since writing this paper the authors have learned from Dr. H. Müntz that the new Einstein field equations of the 1929 paper do not yield the vanishing of the gravitational field in the case of spherical symmetry and time symmetry. In this case he has been able to obtain results checking the observed perihelion of mercury" ([416], p. 356)

Müntz is mentioned in $[88,85]$.

In his paper "On unified field theory" of January 1929, Einstein acknowledges work of a Mr. Müntz:

"I am pleased to dutifully thank Mr. Dr. H. Müntz for the laborious exact calculation of the centrally-symmetric problem based on the Hamiltonian principle; by the results of this investigation I was led to the discovery of the road following here." ${ }^{247}$

Again, two months later in his next paper, "Unified field theory and Hamiltonian principle", Einstein remarks:

"Mr. Lanczos and Müntz have raised doubt about the compatibility of the field equations obtained in the previous paper [...]."

\footnotetext{
${ }^{244}$ There is no contradiction with the result of Einstein and Mayer [106]; this paper proceeds from different field equations.

245 Norbert Wiener (1894-1964). Born in Columbia, Missouri, U.S.A. Studied at Tufts College and Harvard University and received his doctorate with a dissertation on mathematical logic. He continued his studies in Cambridge, England and in Göttingen. From 1918 instructor at the Massachusetts Institute of Technology where he first studied Brownian motion. Wiener had a wide range of interests, from harmonic analysis to communications theory and cybernetics.

246 Manuel Sandoval Vallarta (1899-1977). Born in Mexico City. He studied at the Massachusetts Institute of Technology (MIT), where he received his degree in science and specialised in theoretical physics (1924). With a scholarship from the Guggenheim Foundation (1927-1928), he studied physics in Berlin and Leipzig. From 1923 to 1946, he worked as an assistant, associate, and regular professor at the MIT, and guest professor at the Lovaina University in Belgium (Cooperation with Lemaitre). From 1943, he divided his time between MIT and the School of Sciences and the Institute of Physics of the National Autonomous University of Mexico (UNAM). His main contributions were in mathematic methods, quantum mechanics, general relativity and, from 1932, cosmic rays.

247 "Es ist mir eine angenehme Pflicht, Hrn. Dr. H. Müntz für die mühsame strenge Berechnung des zentralsymmetrischen Problems auf Grund des Hamiltonschen Prinzips zu danken; durch die Ergebnisse jener Untersuchung wurde mir die Auffindung des hier beschrittenen Weges nahegebracht."
} 
and, by deriving field equations from a Lagrangian shows that the objection can be overcome. In his paper in July 1929, the physicist Zaycoff had some details:

"Solutions of the field equations on the basis of the original formulation of unified field theory to first approximation for the spherically symmetric case were already obtained by Müntz."

In the same paper, he states: "I did not see the papers of Lanczos and Müntz." Even before this, in the same year, in a footnote to the paper of Wiener and Vallarta, we read:

"Since writing this paper the authors have learned from Dr. H. Müntz that the new Einstein field equations of the 1929 paper do not yield the vanishing of the gravitational field in the case of spherical symmetry and time symmetry. In this case he has been able to obtain results checking the observed perihelion of mercury."

The latter remark refers to a constant query Pauli had about what would happen, within unified field theory, to the gravitational effects in the planetary system, described so well by general relativity $^{248}$.

Unfortunately, as noted by Meyer Salkover of the Mathematics Department in Cincinatti, the calculations by Wiener and Vallarta were erroneous; if corrected, one finds the Schwarzschild metric is indeed a solution of Einstein's field equations. In the second of his two brief notes, Salkover succeeded in gaining the most general, spherically symmetric solution [288, 287]. This is admitted by the authors in their second paper, in which they present a new calculation.

"In a previous paper the authors of the present note have treated the case of a spherically symmetrical statical field, and stated the conclusions: first, that under Einstein's definition of the electromagnetic potential an electromagnetic field is incompatible with the assumption of static spherical symmetry and symmetry of the past and future; second, that if one uses the Hamiltonian suggested in Einstein's second 1928 paper, the electromagnetic potential vanishes and the gravitational field also vanishes."

And they hasten to reassure the reader:

"None of the conclusions of the previous paper are vitiated by this investigation, although some of the final formulas are supplemented by an additional term." ([417], p. 802$)$

Vallarta also wrote a paper by himself ([358], p. 784) whose abstract reads:

"In recent papers Wiener and the author have determined the tensors ${ }^{s} h_{\lambda}$ of Einstein's unified theory of electricity and gravitation under the assumption of static spherical symmetry and of symmetry of past and future. It was there shown that the field equations suggested in Einstein's second 1928 paper [83] lead in this case to a vanishing gravitational field. The purpose of this paper is to investigate, for the same case, the nature of the gravitational field obtained from the field equations suggested by Einstein in his first 1929 paper [88]."

He also claims

"that Wiener has shown in a paper to be published elsewhere soon that the Schwarzschild solution satisfies exactly the field equations suggested by Einstein in his second 1929 paper ([85])."

${ }^{248}$ See Pauli's note of 1919 concerning Weyl's theory [243]. So far, I have not been able to find a publication by Dr. H. Müntz reporting about his calculations for Einstein. What should we conclude if none exists? That Einstein lost his interest in this particular version of unified field theory? That the calculations were erroneous, or just not reaching far enough? Further hypotheses are possible.

Living Reviews in Relativity

http: //www. livingreviews.org/lrr-2004-2 
Finally, Rosen and Vallarta [283] got together for a systematic investigation of the spherically symmetric, static field in Einstein's unified field theory of the electromagnetic and gravitational fields [93].

Further papers on Einstein's teleparallelism theory were written in Italy by Bortolotti in Cagliari, Italy [22, 23, 25, 24], and by Palatini [242].

In Princeton, people did not sleep either. In 1930 and 1931, T. Y. Thomas wrote a series of six papers on distant parallelism and unified field theory. He followed Einstein's example by also changing his field equations from the first to the second publication. After that, he concentrated on more mathematical problems, such as proving an existence theorem for the Cauchy-Kowlewsky type of equations in unified field theory, by studying the characteristics and bi-characteristic, the characteristic Cauchy problem, and Huygen's principle. T. Y. Thomas described the contents of his first paper as follows:

"In a number of notes in the Berlin Sitzungsberichte followed by a revised account in the Mathematische Annalen, Einstein has attempted to develop a unified theory of the gravitational and electromagnetic field by introducing into the scheme of Riemannian geometry the possibility of distant parallelism. [...] we are led to the construction of a system of wave equations as the equations of the combined gravitational and electromagnetic field. This system is composed of 16 equations for the determination of the 16 quantities $h_{i}^{\hat{k}}$ and is closely analogous to the system of 10 equations for the determination of the 10 components $g_{i k}$ in the original theory of gravitation. It is an interesting fact that the covariant components $h_{i}^{\hat{k}}$ of the fundamental vectors, when considered as electromagnetic potential vectors, satisfy in the local coordinate system the universally recognised laws of Maxwell for the electromagnetic field in free space, as a consequence of the field equations." [350]

This looks as if he had introduced four vector potentials for the electromagnetic field, and this, in fact, T. Y. Thomas does: "the components $h_{i}^{\hat{k}}$ will play the role of electromagnetic potentials in the present theory." The field equations are just the four wave equations $\sum e_{\hat{k}} h_{\hat{\jmath}, \hat{k} \hat{k}}$ where the summation extends over $\hat{k}$, with $\hat{k}=1, \ldots, 4$, and the comma denotes an absolute derivative he has introduced. The gravitational potentials are still $g_{i k}$. In his next note, T. Y. Thomas changed his field equations on the grounds that he wanted them to give a conservation law.

"This latter point of view is made the basis for the construction of a system of field equations in the present note - and the equations so obtained differ from those of note I only by the appearance of terms quadratic in the quantities $h_{j, k}^{\hat{\imath}}$. It would thus appear that we can carry over the interpretation of the $h_{i}^{\hat{k}}$ as electromagnetic potentials; doing this, we can say that Maxwell's equations hold approximately in the local coordinate system in the presence of weak electromagnetic fields." [351]

The third paper contains a remark as to the content of the concept "unified field theory":

"It is the objective of the present note to deduce the general existence theorem of the Cauchy-Kowalewsky type for the system of field equations of the unified field theory. [...] Einstein (Sitzber. 1930, 18-23) has pointed out that the vanishing of the invariant $h_{j, k}^{\hat{\imath}}$ is the condition for the four-dimensional world to be Euclidean, or more properly, pseudo-Euclidean. From the point of view of our previous notes this fact has its interpretation in the statement that the world will be pseudo-Euclidean only in the absence of electric and magnetic forces. This means that gravitational and electromagnetic phenomena must be intimately related since the existence of gravitation becomes dependent on the electromagnetic field. Thus we secure a real physical unification of 
gravitation and electricity in the sense that these concepts become but different manifestations of the same fundamental entity - provided, of course, that the theory shows itself to be tenable as a theory in agreement with experience." [352].

In his three further installments, T. Y. Thomas moved away from unified field theory to the discussion of mathematical details of the theory he had advanced [353, 354, 355].

Unhindered by constraints from physical experience, mathematicians try to play with possibilities. Thus, it was only consequential that Valentin Bargmann in Berlin, after Riemann and Weyl, now engaged in looking at a geometry allowing a comparison "at a distance" of directions but not of lengths, i.e., only of the quotient of vector components, $A^{i} / A^{k}[5]$. In the framework of a purely affine theory he obtained a necessary and sufficient condition for this geometry,

$$
R_{j k l}^{i}(\Gamma)=\frac{1}{D} \delta^{i}{ }_{j} V_{k l}
$$

with the homothetic curvature $V_{k l}$ from Equation (31). Then Bargmann linked his approach to Einstein's first note on distant parallelism [84, 89], introduced a $D$-bein $h_{i}^{k}$, and determined his connection such that the quotients $A^{i} / A^{k}$ of vector components with regard to the $D$-bein remained invariant under parallel transport. The resulting connection is given by

$$
\Gamma_{l m}^{k}=h_{j}^{k} \frac{\partial h_{l}^{j}}{\partial x^{m}}-\delta_{l}^{k} \psi_{m}
$$

where $\psi_{m}$ corresponds to $\Gamma_{m k}$.

Schouten and van Dantzig also used a geometry built on complex numbers, and on Hermitian forms:

"[...] we were able to show that the metric geometry used by Einstein in his most recent approach to relativity theory $[84,83]$ coincides with the geometry of a Hermitian tensor of highest rank, which is real on the real axis and satisfies certain differential equations." ([313], p. 319)

The Hermitian tensor referred to leads to a linear integrable connection that, in the special case that it "is real in the real", coincides with Einstein's teleparallel connection.

Distant parallelism was revived four decades later within the framework of Poincaré gauge theory; the corresponding theories will be treated in the second part of this review.

\subsubsection{Overdetermination and compatibility of systems of differential equations}

In the course of Einstein's thinking about distant parallelism, his ideas about overdetermined systems of differential equations gradually changed. At first, the possibility of gaining hold on the paths of elementary particles - described as singular worldlines of point particles - was central. He combined this with the idea of quantisation, although Planck's constant $h$ could not possibly surface by such an approach. But somehow, for Einstein, discretisation and quantisation must have been too close to bother about a fundamental constant.

Then, after the richer constructive possibilities (e.g., for a Lagrangian) became obvious, a principle for finding the correct field equations was needed. As such, "overdetermination" was brought into the game by Einstein:

"The demand for the existence of an 'overdetermined' system of equations does provide us with the means for the discovery of the field equations" ${ }^{249}$ ([90], p. 21)

\footnotetext{
249 "Die Forderung der Existenz eines 'überbestimmten' Gleichungssystems mit der erforderlichen Zahl der Iden-
} titäten gibt uns ein Mittel zur Auffindung der Feldgleichungen an die Hand."

Living Reviews in Relativity

http://www. livingreviews.org//rr-2004-2 
It seems that Einstein, during his visit to Paris in November 1929, had talked to Cartan about his problem of finding the right field equations and proving their compatibility. Starting in December of 1929 and extending over the next year, an intensive correspondence on this subject was carried on by both men [50]. On 3 December 1929, Cartan sent Einstein a letter of five pages with a mathematical note of 12 pages appended. In it he referred to his theory of partial differential equations, deterministic and "in involution," which covered the type of field equations Einstein was using and put forward a further field equation. He clarified the mathematical point of view but used concepts such as "degree of generality" and "generality index" not familiar to Einstein ${ }^{250}$. Cartan admitted ${ }^{251}$ :

"I was not able to completely solve the problem of determining if there are systems of 22 equations other than yours and the one I just indicated [...] and it still astonishes me that you managed to find your 22 equations! There are other possibilities giving rise to richer geometrical schemes while remaining deterministic. First, one can take a system of 15 equations [...]. Finally, maybe there are also solutions with 16 equations; but the study of this case leads to calculations as complicated as in the case of 22 equations, and I was not fortunate enough to come across a possible system [...]." ([50], pp. 25-26)

Einstein's rapid answer of 9 December 1929 referred to the letter only; he had not been able to study Cartan's note. As the further correspondence shows, he had difficulties in following Cartan:

"For you have exactly that which I lack: an enviable facility in mathematics. Your explanation of the indice de généralité I have not yet fully understood, at least not the proof. I beg you to send me those of your papers from which I can properly study the theory." ([50], p. 73)

It would be a task of its own to closely study this correspondence; in our context, it suffices to note that Cartan wrote a special note ${ }^{252}$

"[...] edited such that I took the point of view of systems of partial differential equations and not, as in my papers, the point of view of systems of equations for total differentials $[\ldots]^{\prime 253}$

which was better suited to physicists. Through this note, Einstein came to understand Cartan's theory of systems in involution:

"I have read your manuscript, and this enthusiastically. Now, everything is clear to me. Previously, my assistant Prof. Müntz and I had sought something similar - but we were unsuccessful." 254 ([50], pp. 87, 94)

In the correspondence, Einstein made it very clear that he considered Maxwell's equations only as an approximation for weak fields, because they did not allow for non-singular exact solutions approaching zero at spacelike infinity.

${ }^{250}$ Cartan explained the generality index like this:

"[...] the general solution of a given deterministic system in involution depends on $r_{2}+2 p-n$ arbitrary functions of three variables in the sense that the 3 -dimensional solution $\left(x_{4}=0\right)$ that determines the most general solution can be obtained by arbitrarily taking $r_{2}+2 p-n$ of the unknown functions to be functions of $x_{1}, x_{2}, x_{3} . "$

Here, $n$ is the number of equations of the system for $p$ unknown functions; $r_{2}$ denotes the number of existing linear relations among the $2 p$ derivatives of the unknowns with regard to two arbitrary directions in 3 -space.

${ }^{251}$ Here, and in the sequel, I mostly take over the English translation by J. Leroy and J. Ritter used in the book. ${ }^{252}$ Cartan's note was published in a very similar form in [37].

253 “[...] redigée en me placant au point de vue des systèmes d'équations aux dérivées partielles et non, comme dans mes mémoires, au point de vue des systémes d'équations aux différentielles totales [...]"

254 "Ich habe Ihr Manuskript gelesen und zwar mit Begeisterung. Nun ist mir alles klar. [...] Ich hatte mit meinem Assistenten Prof. Müntz früher etwas ähnliches versucht - wir sind aber nicht durchgekommen." 
"It now is my conviction that for rigourous field theories to be taken seriously everywhere a complete absence of singularities of the field must be demanded. This probably will restrict the free choice of solutions in a region in a far-reaching way - more strongly than the restrictions corresponding to your degrees of determination." ${ }^{255}$ ([50], p. 92)

Although Einstein was grateful for Cartan's help, he abandoned the geometry with distant parallelism.

\footnotetext{
255 "Es ist nun meine Überzeugung, dass bei ernst zu nehmenden Feldtheorien völlig Singularitätsfreiheit des ganzen Feldes verlangt werden muss. Dies wird wohl die freie Wahl der Lösungen in einem Gebiete in einer sehr weitgehenden Weise einschränken - über die Einschränkung hinaus, die Ihren Determinationsgrade entsprechen.”
} 


\section{Geometrization of the Electron Field as an Additional Element of Unified Field Theory}

After the advent of Schrödinger's and Dirac's equations describing the electron non-relativistically and relativistically, a unification of only the electromagnetic and gravitational fields was considered unconvincing by many theoretical physicists. Hence, in the period 1927-1933, quite a few attempts were made to include Schrödinger's, Dirac's, or the Klein-Gordon equation as a classical oneparticle equation into a geometrical framework by relating the quantum mechanical wave function with some geometrical object. Such an approach then was believed to constitute a unification, up to a degree, of gravitation and/or electricity and quantum theory. In this section, we loosely collect some of these approaches.

The mathematicians Struik and Wiener found the task of an amalgamation of relativity and quantum theory (wave mechanics) attractive:

"It is the purpose of the present paper to develop a form of the theory of relativity which shall contain the theory of quanta, as embodied in Schrödingers wave mechanics, not merely as an afterthought, but as an essential and intrinsic part." [338]

A further example for the new program is given by J. M. Whittaker at the University of Edinburgh [415] who wished to introduce the wave function via the matter terms:

"In addition to the wave equations a complete scheme must include electromagnetic and gravitational equations. These will differ from the equations of Maxwell and Einstein in having 'wave' terms instead of 'particle' terms for the current vector and material energy tensor. The object of the present paper is to find these equations [...]." ([415], p. 543)

Zaycoff, from the point of view of distant parallelism, found the following objection to unified field theory as the only valid one:

"It neglects the existence of wave-mechanical phenomena. By the work of Dirac, wavemechanics has reached an independent status; the only attempt to bring together this new group of phenomena with the other two is J. M. Whittaker's theory [415]." ${ }^{256}$ [428]

In fact, in a short note, Zaycoff presented his version of Whittaker's theory with 8 coupled secondorder linear field equations for two "wave vectors" that, in a suitable combination, were to represent "the Dirac's wave equations"; they contain the Ricci tensor and both the electromagnetic 4-potential and field [432]. Thus, what is described is Dirac's equation in external gravitational and electromagnetic fields, not a unified field theory. Whittaker had expressed himself more clearly:

"Eight wave functions are employed instead of Dirac's four. These are grouped together to form two four-vectors and satisfy wave equations of the second order. It is shown [...] that these eight wave equations can be reduced, by addition and subtraction, to the four second order equations satisfied by Dirac's functions taken twice over; and that, in a sense, the present theory includes Dirac's." ([415], p. 543)

Whittaker also had written down a variational principle by which the gravitational and electromagnetic field equations were also gained. However, as the terms for the various fields were just added up in his Lagrangian, the theory would not have qualified as a genuine unified field theory in the spirit of Einstein.

256 "Sie vernachlässigt die Existenz der wellenmechanischen Erscheinungen. Die Wellenmechanik ist durch die Arbeiten von Dirac in eine selbständige Phase getreten, und der einzige Versuch, diese neue Gruppe von Erscheinungen mit den übrigen beiden in Zusammenhang zu bringen, ist die Theorie von J. M. Whittaker [415]." 
What fancy, if only shortlived, flowers sprang from the mixing of geometry and wave mechanics is shown by the example of H. Jehle's

"[...] path leading, on the one hand, to electrical elementary particles and, on the other, to the explanation of cosmological problems by quantum theory." [171]

His ad-hoc modification of Einstein's equations was:

$$
G_{i k} \psi^{2}-\sigma^{2} R \frac{\partial \psi}{\partial x^{i}} \frac{\partial \psi}{\partial x^{k}}=0, \quad G_{i k} \psi \bar{\psi}-\sigma^{2} R \frac{\partial \psi}{\partial x^{i}} \frac{\partial \bar{\psi}}{\partial x^{k}}=0,
$$

where $\psi$ is the quantum mechanical state function. Although, two years later, Jehle withdrew his claims concerning elementary particles, he continued to apply

"wave-mechanical methods to gravitational phenomena, by which the curious structure of the spiral nebulae and spherical star systems may be readily understood." [172]

An eminent voice was Weyl's:

"It seems to me that it is now hopeless to seek a unification of gravitation and electricity without taking material waves into account." ([408], p. 325)

Now, this posed a problem because for the representation of the electrons in the form of Dirac's equation, the elements of spin space, i.e., spinors, had to be used. How to combine them with the vectors and tensors appearing in electromagnetic and gravitational theories? As the spinor representation is the simplest representation of the Lorentz group, everything may be played back to spin space. At the time, this was being done in different ways, in part by the use of number fields with which physicists were unacquainted such as quaternions and sedenions (cf. Schouten [315]). Others, such as Einstein and Mayer, liked vectors better and introduced so-called semi-vectors. Still others tried to write Dirac's equations in a vectorial form and took into account the doubling of variables and equations [213, 415]. Some less experienced, as e.g., "Exhibition Research Student" G. Temple, even claimed that a tensorial theory was necessary to retain it relativistically:

'It is an admitted fact that Dirac's wave functions are not the components of a tensor and that his wave equations are not in tensorial form. It is contended here that therefore his theory cannot be upheld without abandoning the theory of relativity." ([343], p. 352)

While this story about geometrizing wave mechanics might not be a genuine part of unified field theory at the time, it seems interesting to follow it as a last attempt for binding together classical field theory and quantum physics. Even Einstein was lured into thinking about spinors by Dirac's equation; this equation promised more success for his program concerning elementary particles as solutions of differential equations (cf. Section 7.3. )

\subsection{Unification of Maxwell's and Dirac's equations, of electrons and light}

The appearance of these so-called "wave equations" for some seemed to show a kinship between the photon and the electron; this led to attempts to obtain a common representation for both kind of particles (waves) [199, 200, 198, 286]. Some of the motivation for these papers came from formal considerations, i.e., the wish to replace the not yet well-understood new spinor representation of the Lorentz group by the old tensor representation; a working knowledge about non-commuting objects like matrices was not yet available to everyone:

"There are probably readers who will share the present writer's feeling that the methods of non-commutative algebra are harder to follow, and certainly much more difficult to invent, than are operations of types long familiar to analysis." ([44], p. 654)

Living Reviews in Relativity

http: //www. livingreviews.org/lrr-2004-2 
More interesting is Frenkel's remark about Darwin's presentation of Dirac's equations in a form analogous to Maxwell's equations [44]:

"This relation between the wave-mechanical equations of a 'quantum of electricity' and the electromagnetic field equations, which may be looked at as wave-mechanical equations for photons, ought to have a fundamental physical meaning. Therefore, I do not think it is superfluous to win the wave equation of the electron as a generalisation of M axw e l l's equations." 257 ([140], p. 357)

H. T. Flint of King's College in London aimed at describing the electron in a Maxwell-like way within a five-dimensional approach. He saw two "unsatisfactory points" in Dirac's approach, the introduction of the operator $\frac{h}{2 \pi i} \frac{\partial}{\partial x^{k}}-e \phi_{k}$, and the mass term $m c$. In order to mend these thin spots he wrote down two Maxwell's equations,

$$
\nabla_{\mu} F^{\lambda \mu}=J^{\lambda}, \quad \nabla_{\mu} G^{\lambda \mu}=L^{\lambda},
$$

where $F^{\lambda \mu}$ and $G^{\lambda \mu}$ are two asymmetric field tensors, and $J_{\lambda}=\frac{\partial \psi}{\partial x^{\lambda}}$ and $L_{\lambda}=\frac{\partial \theta}{\partial x^{\lambda}}$ are two current vectors $(\mu, \lambda, \nu=1,2, \ldots, 5)$. $\psi$ is the electron's wave function; although not provided by Flint, the interpretation of $\theta$ points to a second kind of wave function. Despite the plentitude of variables introduced, Flint's result was meagre; his second order wave equation contained the correct mass term and two new terms; he did not write up all the equations occurring [129]. His approach for embedding wave mechanics into a Maxwell-like was continued in further papers, in part in collaboration with J. W. Fisher; to them it appeared

"unnecessary to introduce in any arbitrary way terms and operators to account for quantum phenomena." ([128], p. 653; [127])

By adding four spinor equations at his choosing to Dirac's equation, Wisniewski in Poland arrived at a "system of equations similar to Maxwell's". His conclusion sounds a bit strange:

"These equations may be interpreted as equations for the electromagnetic field in an electron gas whose elements are electric and magnetic dipoles." [388]

In this context, another unorthodox suggestion was put forward by A. Anderson who saw matter and radiation as two phases of the same substrate:

"We conclude that, under sufficiently large pressure, even at absolute zero normal matter and black-body radiation (gas of light quanta) become identical in every sense. Electrons and protons cannot be distinguished from quanta of light, gas pressure not from radiation pressure." 258

Anderson somehow sensed that charge conservation was in his way; he meddled through by either assuming neutral matter, i.e., a mixture of electrons and protons, or by raising doubt as to "whether the usual quanta of light are strictly electrically neutral" ([3], p. 441).

One of the German theorists trying to keep up with wave mechanics was Gustav Mie. He tried to reformulate electrodynamics into a Schrödinger-type equation and arrived at a linear, homogeneous wave equation of the Klein-Gordon-type for the $\psi$-function on the continuum of the

\footnotetext{
257 "Diese Beziehung zwischen den wellenmechanischen Gleichungen eines 'Elektrizitätsquantums' und den elektromagnetischen Feldgleichungen, die man als wellenmechanische Gleichungen von Lichtquanten ansehen darf, muss wahrscheinlich eine grundlegende physikalische Bedeutung haben. Es erscheint mir daher nicht überflüssig [...], die Wellengleichung des Elektrons als Verallgemeinerung der M a x w e l lschen Gleichungen aufzustellen."

258 "Wir kommen also zu dem Schluss, dass unter genügend grossem Drucke selbst beim absoluten Nullpunkt gewöhnliche Materie und Hohlraumstrahlung (Lichtquantengas) in jeder Hinsicht identisch werden. Die Elektronen und Protonen sind nicht zu unterscheiden von Lichtquanten, der Gasdruck nicht vom Strahlungsdruck."
} 
components of the electromagnetic vector potential [232]. Heisenberg and Pauli, in their paper on the quantum dynamics of wave fields, although acknowledging Mie's theory as an attempt to establish the classical side for the application of the correspondence principle, criticised it as a formal scheme not yet practically applicable [158].

\subsection{Dirac's electron with spin, Einstein's teleparallelism, and Kaluza's fifth dimension}

In the same year 1928 in which Einstein published his theory of distant parallelism, Dirac presented his relativistic, spinorial wave equation for the electron with spin. This event gave new hope to those trying to include the electron field into a unified field theory; it induced a flood of papers in 1929 such that this year became the zenith for publications on unified field theory. Although we will first look at papers which gave a general relativistic formulation of Dirac's equation without having recourse to a geometry with distant parallelism, Tetrode's paper seems to be the only one not influenced by Einstein's work with this geometry (cf. Section 6.4.5). Although, as we noted in Section 6.4.1, the technique of using $n$-beins (tetrads) had been developed by mathematicians before Einstein applied it, it may well have been that it became known to physicists through his work. Both Kaluza's five-dimensional space and four-dimensional projective geometry were also applied in the general relativistic formulation of Dirac's equation.

\subsubsection{Spinors}

This is a very sketchy outline with a focus on the relationship to unified field theories. An interesting study into the details of the introduction of local spinor structures by Weyl and Fock and of the early history of the general relativistic Dirac equation was given recently by Scholz [291].

For some time, the new concept of spinorial wave function stayed unfamiliar to many physicists deeply entrenched in the customary tensorial formulation of their equations ${ }^{259}$. For example, J. M. Whittaker was convinced that Dirac's theory for the electron

"has been brilliantly successful in accounting for the 'duplexity' phenomena of the atom, but has the defect that the wave equations are unsymmetrical and have not the tensor form." ([415], p. 543)

Some early nomenclature reflects this unfamiliarity with spinors. For the 4-component spinors or Dirac-spinors (cf. Section 2.1.5) the name "half-vectors" coined by Landau was in use ${ }^{260}$. Podolsky even purported to show that it was unnecessary to employ this concept of "half-vector" if general curvilinear coordinates are used [259]. Although van der Waerden had written on spinor analysis as early as 1929 [368] and Weyl's [407, 408], Fock's [133, 131], and Schouten's [306] treatments in the context of the general relativistic Dirac equation were available, it seems that only with van der Waerden's book [369], Schrödinger's and Bargmann's papers of 1932 [319, 6], and the publication of Infeld and van der Waerden one year later [167] a better knowledge of the new representations of the Lorentz group spread out. Ehrenfest, in 1932, still complained ${ }^{261}$ :

"Yet still a thin booklet is missing from which one could leasurely learn spinor- and tensor-calculus combined." ${ }^{262}$ ([68], p. 558)

\footnotetext{
${ }^{259}$ Although the name "spinor" seems to have been suggested by Ehrenfest, Cartan first used the concept in 1913

${ }^{260}$ Scholz uses the expression "semi-vectors" which is reserved here for the objects of Einstein and Mayer with same name (cf. Section 7.3).

${ }^{261}$ Ehrenfest had uttered much the same complaint to van der Waerden who had tried to answer to it by this paper, obviously without satisfying Ehrenfest but nevertheless laying the ground for other physicists' understanding.

262 "Noch immer fehlt ein dünnes Büchlein, aus dem man gemütlich die Spinorrechnung mit der Tensorrechnung vereinigt lernen könnte."
}

Living Reviews in Relativity

http: //www. livingreviews.org/lrr-2004-2 
In 1933, three publications of the mathematician Veblen in Princeton on spinors added to the development. He considered his first note on 2-spinors "a sort of geometric commentary on the paper of Weyl" [378]. Veblen had studied Weyl's, Fock's, and Schouten's papers, and now introduced a "spinor connection of the first kind" $\Lambda_{B \alpha}^{A}, \alpha=1, \ldots, 4$, with the usual transformation law under the linear transformation $\bar{\psi}^{A}=T_{D}^{A} \psi^{D}(A, D=1,2)$ changing the spin frame:

$$
\bar{\Lambda}_{D \alpha}^{C}=\left(\Lambda_{B \alpha}^{A} t_{D}^{B}+\frac{\partial t_{D}^{A}}{\partial x^{\alpha}}\right) T_{A}^{C},
$$

where $t_{D}^{B}$ is the inverse matrix $T^{-1} . T_{D}^{A}$ corresponds to $A_{B}^{A}$ of Equation (75); however, the transformation need not be unimodular. Thus, Veblen took up Schouten's concept of "spin density" [306] by considering quantities transforming like $\bar{\psi}^{A}=t^{N} T_{D}^{A} \psi^{D}(A, D=1,2)$, with $t:=\operatorname{det} t_{D}^{B}$. Then, the covariant derivative of a spinor of weight $N, \psi^{A}$ is considered; the expression ${ }^{263}$

$$
\frac{\partial \psi^{A}}{\partial x^{\alpha}}+\Lambda_{B \alpha}^{A} \psi^{B}-N \Lambda_{B \alpha}^{B} \psi^{A}=\nabla_{\alpha} \psi^{A}
$$

gives "the components of a geometric object which transforms like those of a spinor of weight $N$ with respect to the index $A$ and like those of a projective tensor with respect to $\alpha$ ". In his first paper [378], a further generalisation is introduced including "gauge-transformations" in an additional variable $x^{0}: \bar{\psi}^{A}=e^{k x^{0}} f^{A}(A, D=1,2)$, with the gauge transformations

$$
x^{0}=x^{0^{\prime}}-\log \rho\left(x^{k^{\prime}}\right), \quad x^{k}=x^{k}\left(x^{l^{\prime}}\right),
$$

$k$ is called the "index" of the spinor. In order to deal with 4-spinors Veblen considered a complex projective 3-space and defined 6 real homogeneous coordinates $X^{\sigma}$, with $\sigma=0, \ldots, 5$, through Hermitian forms of the 4-spinor components. The subspace $X^{0}=0, X^{5}=0$ of the quadratic $\left(X^{0}\right)^{2}+\left(X^{1}\right)^{2}+\left(X^{2}\right)^{2}+\left(X^{3}\right)^{2}-\left(X^{4}\right)^{2}-\left(X^{5}\right)^{2}=0$ is then tangent to the Minkowski light cone ([377], p. 515).

Veblen imbedded spinors into his projective geometry [380]:

"[...] The components of still other objects, the spinors, remain partially indeterminate after coordinates and gauge are fixed and become completely determinate only when the spin frame is specified. There are several ways of embodying this invariant theory in a formal calculus. The one which is here employed has its antecedents chiefly in the work of Weyl, van der Waerden, Fock, and Schouten. It differs from the calculus arrived at by Schouten chiefly in the treatment of gauge invariance, Schouten (in collaboration with van Dantzig) having preferred to rewrite the projective relativity in a formalism obtainable from the original one by a sort of coordinate transformation, whereas I think the original form fits in better with the classical notations of relativity theory. [...] The theory of spinors is more general than the projective relativity and is reduced to the latter by the specification of certain fundamental spinors. These spinors have been recognised by several students (Pauli and Solomon, Fock) of the subject but their role has probably not been fully understood since it has quite recently been thought necessary to give special proofs of invariance." [380]

The transformation law for spinors is the same as before ${ }^{264}$ :

$$
\bar{\psi}=e^{k x^{0}} t^{N} T_{B}^{A} \psi^{B}, \quad A, B=1, \ldots, 4 .
$$

${ }^{263}$ While Veblen denoted the covariant derivative of $\psi^{A}$ by $\psi_{, \alpha}^{A}$, we are using the nabla-symbol.

${ }^{264}$ Unfortunately, in his paper, in contrast to his previous and our notation, Veblen now used $N$ for the index and $k$ for weight. 
In part, he also takes over van der Waerden's notation (dotted indices.) As to Veblen's papers on 2- and 4-spinors, my impression is that, beyond a more detailed presentation, alas with a less transparent notation, they do not really bring a pronounced advance with regard to Weyl's, Fock's, van der Waerden's, and Pauli's publications (cf. Sections 7.2.2 and 7.2.3). Veblen himself had a different opinion; for him the homogeneous coordinates used by Pauli seemed "to make things more complicated" (cf. the paragraphs on projective geometry in Section 2.1.3). Veblen's inhomogeneous coordinates $x^{i},(i=1,2,3,4)$ and the homogeneous coordinates $X^{\mu}$, with $\mu=$ $0, \ldots, 4$, are connected by

$$
X^{0}=\exp \left(x^{0}\right), \quad x^{i}=\exp \left(x^{0} x^{i}\right)
$$

According to Veblen,

"In a five-dimensional representation the use of the homogeneous coordinates $\left(X^{0}, \ldots, X^{4}\right)$ amounts to representing the points of space-time by the straight lines through the origin, whereas the use of $x^{1}, \ldots, x^{4}$, and the gauge variable amounts to using the system of straight lines parallel to the $x^{0}$-axis for the same purpose. The transformation (192) given above carries the system of lines into the other." [382]

\subsubsection{General relativistic Dirac equation and unified field theory}

After Tetrode and Wigner, whose contributions were mentioned in Section 6.4.5, Weyl also gave a general relativistic formulation of Dirac's equation. He gave up his original idea of coupling electromagnetism to gravitation and transferred it to the coupling of the electromagnetic field to the matter (electron-) field: In order to keep quantum mechanical equations like Dirac's gauge invariant, the wave function had to be multiplied by a phase factor [407, 408]. Actually, Weyl had expressed the change in his outlook, so important for the idea of gauge-symmetry in modern physics ([424], pp. 13-19), already in 1928 in his book on group theory and quantum mechanics ([406], pp. 87-88). We have noted before his refutation of distant parallelism (cf. Section 6.4.4). In his papers, Weyl used a 2-spinor formalism and a tetrad notation different from Einstein's and Levi-Civita's: He wrote $e^{p}(\hat{k})$ in place of $h_{\hat{k}}^{p}$, and $o(l ; k j)$ for the Ricci rotation coefficients $\gamma_{j k l}$; this did not ease the reading of his paper. He partly agreed with what Einstein imagined:

"It is natural to expect that one of the two pairs of components of D i r a c's quantity belongs to the electron, the other to the proton." 265

In contrast to Einstein, Weyl did not expect to find the electron as a solution of "classical" spinorial equations:

"For every attempt at establishing the quantum-theoretical field equations, one must not lose sight [of the fact] that they cannot be tested empirically, but that they provide, only after their quantization, the basis for statistical assertions concerning the behaviour of material particles and light quanta." 266 ([407], p. 332)

For many years, Weyl had given the statistical approach in the formulation of physical laws an important role. He therefore could adapt easily to the Born-Jordan-Heisenberg statistical interpretation of the quantum state. For Weyl and statistics, cf. Section V of Sigurdsson's dissertation ([326], pp. 180-192).

At about the same time, Fock in May 1929 and later in the year wrote several papers on the subject of "geometrizing" Dirac's equation:

\footnotetext{
265 "Es ist naheliegend, zu erwarten, dass von den beiden Komponentenpaaren der D i r a cschen Grösse das eine dem Elektron, das andere dem Proton zugehört."

266 "Bei jedem Versuch zur Aufstellung der quantentheoretischen Feldgleichungen muss man im Auge haben, dass diese nicht direkt mit der Erfahrung verglichen werden können, sondern erst nach ihrer Quantisierung die Unterlage liefern für die statistischen Aussagen über das Verhalten der materiellen Teilchen und Lichtquanten."
} 
"In the past two decades, endeavours have been made repeatedly to connect physical laws with geometrical concepts. In the field of gravitation and of classical mechanics, such endeavours have found their fullest accomplishment in E i n s t e i n's general relativity. Up to now, quantum mechanics has not found its place in this geometrical picture; attempts in this direction (Klein, Fock) were unsuccessful. Only after Dirac had constructed his equations for the electron, the ground seems to have been prepared for further work in this direction." 267 ([135], p. 798)

In another paper [134], Fock and Ivanenko took a first step towards showing that Dirac's equation can also be written in a generally covariant form. To this end, the matrix-valued linear form $d s=\gamma_{k} d x^{k}$ (summation over $k=1, \ldots, 4$ ) was introduced and interpreted as the distance between two points "in a space with four continuous and one discontinuous dimensions"; the discrete variable took only the integer values $1,2,3$, and 4 . Then the operator-valued vectorial quantity $\gamma_{k} u^{k}$ with the vectorial operator $u^{k}$ and its derivative $\frac{d s}{d \tau}=\gamma_{k} v^{k}$ immediately led to Dirac's equation by replacing $v_{k}$ by $\frac{1}{m}\left(\frac{h}{2 \pi i} \frac{\partial}{\partial x^{k}}+\frac{e}{c} A_{k}\right)$, where $A_{k}$ is the electromagnetic 4-potential, by also assuming the velocity of light $c$ to be the classical average of the " 4 -velocity" $v_{k}$, and by applying the operator to the wave function $\psi$. In the next step, instead of the Dirac $\gamma$-matrices with constant entries $\gamma_{l}^{(0)}$, the coordinate-dependent bein-components $\gamma_{\hat{k}}:=h_{\hat{k}}^{l} \gamma_{l}^{(0)}$ are defined; $d s^{2}$ then gives the orthonormality relations of the 4-beins.

In a subsequent note in the Reports of the Parisian Academy, Fock and Ivanenko introduced Dirac's 4-spinors under Landau's name "half vector" and defined their parallel transport with the help of Ricci's coefficients. In modern parlance, by introducing a covariant derivative for the spinors, they in fact already obtained the "gauge-covariant" derivative $\nabla_{k} \psi:=\left(\frac{\partial}{\partial x^{k}}-\frac{2 \pi i}{h} \frac{e}{c} A_{k}\right) \psi$. Thus $\delta \psi=\frac{2 \pi i}{h} \frac{e}{c} A_{k} d x^{k} \psi$ is interpreted in the sense of Weyl:

"Thus, it is in the law for the transport of a half-vector that Weyl's differential linear form must appear." 268 ([134], p. 1469)

In order that gauge-invariance results, $\psi$ must transform with a factor of norm 1 , innocuous for observation, i.e., $\psi \rightarrow \exp \left(i \frac{2 \pi}{h} \frac{e}{c} \sigma\right)$ if $A_{k} \rightarrow A_{k}+\frac{\partial \sigma}{\partial x^{k}}$. Another note and extended presentations in both a French and a German physics journal by Fock alone followed suit [133, 131, 132]. In the first paper Fock defined an asymmetric matter tensor for the spinor field,

$$
T^{j}{ }_{k}=\frac{c h}{2 \pi i}\left[\bar{\psi} \gamma^{j}\left(\frac{\partial \psi}{\partial x^{k}}-\Gamma_{k} \psi\right)-\frac{1}{2} \nabla_{k}\left(\bar{\psi} \gamma^{j} \psi\right)\right],
$$

where $\Gamma_{k}=\sum_{\hat{l}} e_{\hat{l}} \alpha_{\hat{l}} h_{k \hat{l}} C_{\hat{l}}$ is related to the matrix-valued spin connection in the expression for the parallel transport of a half-vector $\psi$ :

$$
\delta \psi=\sum_{\hat{l}} e_{\hat{l}} C_{\hat{l}} d s_{\hat{l}} \psi
$$

The covariant derivative then is $D_{k}=\frac{\partial}{\partial x^{k}}-\Gamma_{k}$. Fock made clear that the covariant formulation of Dirac's equation did not need the special geometry of Einstein's theory of distant parallelism:

\footnotetext{
267 "In den letzten zwei Jahrzehnten sind wiederholt Bestrebungen zum Ausdruck gekommen, die physikalischen Gesetze geometrischen Begriffen zuzuordnen. Im Gebiet der Gravitation und der klassischen Mechanik und haben diese Bestrebungen in E i n s t e i ns allgemeiner Relativitätstheorie ihre höchste Vollendung gefunden. Bisher hat aber die Quantenmechanik im geometrischen Bilde keinen Platz gefunden; Versuche in dieser Richtung (Klein, Fock) hatten keinen Erfolg. Erst nachdem Dirac seine Gleichungen für das Elektron aufgestellt hat, scheint der Boden zur weiteren Arbeit in dieser Richtung geschaffen zu sein."

268 "C'est donc dans la loi du déplacement d'un demi-vecteur que doit figurer la forme différentielle lineaire de Weyl."
} 
"By help of the concept of parallel transport of a half-vector, Dirac's equations will be written in a generally invariant form. [...] The appearance of the 4-potential $\phi_{l}$ besides the Ricci-coefficients $\gamma_{i k l}$ in the expression for parallel transport, on the one hand provides a simple reason for the emergence of the term $p_{l}-\frac{e}{c} \phi_{l}$ in the wave equation and, on the other, shows that the potentials $\phi_{l}$ have a place of their own in the geometrical world-view, contrary to Einstein's opinion; they need not be functions of the $\gamma_{i k l} . " 269$ ([131], p. 261, Abstract)

For his calculations, Fock used Eisenhart's book [119] and "the excellent collection of the most important formulas and facts in the paper of Levi-Civita" [207]. Again, Weyl's "principle of gauge invariance" as formulated in Weyl's book of 1928 [406] is mentioned, and Fock stressed that he had found this principle independently and earlier ${ }^{270}$ :

"The appearance of Weyl's differential form in the law for parallel transport of a half vector connects intimately to the fact, observed by the author and also by Weyl (l.c.), that addition of a gradient to the 4-potential corresponds to multiplication of the $\psi$ function with a factor of modulus $1 . " 271$ ([130], p. 266)

The divergence of the complex energy-momentum tensor $W_{k}^{i}=T_{k}^{i}+i U_{k}^{i}$ satisfies

$$
\nabla_{j} T_{k}^{j}=e J^{l} F_{l k}, \quad \nabla_{j} U_{k}^{j}=\frac{h c}{4 \pi} J^{l} R_{l k},
$$

with the electromagnetic field tensor $F_{i k}$, the Ricci tensor $R_{i k}$, and the Dirac current $J^{k}$. The French version of the paper preceded the German "completed presentation"; in it Fock had noted:

"The 4-potential finds its place in Riemannian geometry, and there exists no reason for generalising it (Weyl, 1918), or for introducing distant parallelism (Einstein 1928). In this point, our theory, developed independently, agrees with the new theory by H. Weyl expounded in his memoir 'gravitation and the electron'." ${ }^{272}$ ([132], p. 405)

In both of his papers, Fock thus stressed that Einstein's teleparallel theory was not needed for the general covariant formulation of Dirac's equation. In this regard he found himself in accord with Weyl, whose approach to the Dirac equation he nevertheless criticised:

"The main subject of this paper is 'Dirac's difficulty"273. Nevertheless, it seems to us that the theory suggested by Weyl for solving this problem is open to grave objections; a criticism of this theory is given in our article." 274

\footnotetext{
269 "Mit Hilfe des Begriffs der Parallelübertragung eines Halbvektors werden die Diracschen Gleichungen in allgemein invarianter Form geschrieben. [...] Das Auftreten des Viererpotentials $\phi_{l}$ neben den Riccikoefficienten $\gamma_{i k l}$ in der Formel für die Parallelübertragung gibt einerseits einen einfachen geometrischen Grund für das Auftreten des Ausdrucks $p_{l}-\frac{e}{c} \phi_{l}$ in der Wellengleichung und zeigt andererseits, dass die Potentiale $\phi_{l}$, abweichend von Einsteins Auffassung, einen selbständigen Platz im geometrischen Weltbild haben und nicht etwa Funktionen der $\gamma_{i k l}$ sein müssen."

${ }^{270}$ Fock referred to his paper of 1926 [130].

271 "Das Auftreten der Weylschen Differentialform im Gesetz der Parallelverschiebung eines Halbvektors steht in enger Beziehung mit der vom Verfasser und auch von Weyl (l.c.) bemerkten Tatsache, dass die Addition eines Gradienten zum Viererpotential der Multiplikation der $\psi$-Funktion mit einem Faktor vom absoluten Betrag 1 entspricht." 272 "Le potentiel-vecteur trouve sa place dans la géométrie de Riemann, et on n'a pas besoin de la généraliser (Weyl, 1918) ou d'introduire le parallélisme à distance (Einstein 1928). Dans ce point notre théorie - développée indépendamment - s'accorde avec la nouvelle théorie de $\mathrm{H}$. Weyl exposée dans son mémoire 'Gravitation et Électron'."

${ }^{273}$ By 'Dirac's difficulty' we must understand the existence of negative energy states of the electron and the nonvanishing probability that a change of electrical charge occurs.

274 "L'objet principal de ce mémoire est la 'difficulté de Dirac'. La théorie proposée par Weyl pour résoudre cette difficulté nous semble, cependant, ouverte à de graves objections; une critique de cette théorie est donnée dans notre article cité au debut [this is [135]]."
} 
Weyl's paper is seminal for the further development of the gauge idea [407].

Although Fock had cleared up the generally covariant formulation of Dirac's equation, and had tried to propagate his results by reporting on them at the conference in Charkow in May $1929^{275}$ [169], further papers were written. Thus, Reichenbächer, in two papers on "a wavemechanical 2-component theory" believed that he had found a method different from Weyl's for obtaining Dirac's equation in a gravitational field. As was often the case with Reichenbächer's work, after longwinded calculations a less than transparent result emerged. His mass term contained a square root, i.e., a \pm two-valuedness, which, in principle, might have been instrumental for helping to explain the mass difference of proton and electron. As he remarked, the chances for this were minimal, however [277, 278].

In two papers, Zaycoff (of Sofia) presented a unified field theory of gravitation, electromagnetism and the Dirac field for which he left behind the framework of a theory with distant parallelism used by him in other papers. By varying his Lagrangian with respect to the 4-beins, the electromagnetic potential, the Dirac wave function and its complex-conjugate, he obtained the 20 field equations for gravitation (of second order in the 4-bein variables, assuming the role of the gravitational potentials) and the electromagnetic field (of second order in the 4-potential), and 8 equations of first order in the Dirac wave function and the electromagnetic 4-potential, corresponding to the generalised Dirac equation and its complex conjugate [426, 427].

In another paper, Zaycoff wanted to build a theory explaining the "equilibrium of the electron". This means that he considered the electron as extended. At this occasion, he fought with himself about the admissibility of the Kaluza-Klein approach:

"Recently, repeated attempts have been made to raise the number of dimensions of the world in order to explain its strange lawfulness (H. Mandel, G. Rumer, the author et al.). No doubt, there are weighty reasons for such a seemingly paradoxical view. For it is impossible to represent Poincaré's pressure of the electron within the normal spacetime scheme. However, the introduction of such metaphysical elements is in gross contradiction with space-time causality, although we may doubt in causality in the usual sense due to Heisenberg's uncertainty relations. A multi-dimensional causality cannot be understood as long as we are unable to give the extra dimensions an intuitive meaning." 276 [433]

Rumer's paper is [285] (cf. Section 8). In the paper, Zaycoff introduced a six-dimensional manifold with local coordinates $x_{0}, \ldots, x_{5}$ where $x_{0}, x_{5}$ belong to the additional dimensions. His local 6bein comprises, besides the 4-bein, four electromagnetic potentials and a further one called "eigenpotential" of the electromagnetic field. As he used a "sharpened cylinder condition, " no further scalar field is taken into account. For $x^{0}$ to $x^{4}$ he used the subgroup of coordinate transformations given in Klein's approach, augmented by $x^{5^{\prime}}=x^{5}$.

Schouten seemingly became interested in Dirac's equation through Weyl's publications. He wrote two papers, one concerned with the four-dimensional and a second one with the fivedimensional approach [306, 307]. They resulted from lectures Schouten had given at the Massachusetts Institute of Technology from October to December 1930 and at Princeton University

\footnotetext{
${ }^{275}$ During a discussion with Frenkel, he also insisted that, in Kaluza's approach, the meaning of the fifth coordinate is to be seen in the "preservation of invariance against adding a gradient to the [electromagnetic] vector potential" (p. 651).

276 "Es sind kürzlich wiederholt Versuche gemacht worden (H. Mandel, G. Rumer, Verfasser u. a.), die Dimensionszahl der Welt zwecks Deutung ihrer sonderbaren Gesetzlichkeit zu erhöhen. Es gibt zwar gewichtige Gründe zu einer solchen augenscheinlich paradoxen Anschauung. Denn es ist gar nicht möglich, den Poincaréschen Druck des Elektrons in dem üblichen Raum-Zeitschema darzustellen. Aber die Einführung derartiger metaphysischer Elemente steht in grobem Widerspruch mit der raumzeitlichen Kausalität, obwohl wir andererseits nach der Heisenbergschen Unschärferelation eine Kausalität im üblichen Sinne bezweifeln dürfen. Ein mehrdimensionaler Determinismus ist jedoch unbegreiflich, solange wir nicht in der Lage sind, den überschüssigen Dimensionen einen anschaulichen Sinn beizulegen."
} 
from January to March 1931; Weyl's paper referred to is in Zeitschrift für Physik [407]. Schouten relied on his particular representation of the Lorentz group in a complex space, which later attracted Schrödinger's criticism. [305]. His comment on Fock's paper [131] is ${ }^{277}$ :

"Fock has tried to make use of the indetermination of the displacement of spin-vectors to introduce the electromagnetic vector potential. However the displacement of contravariant tensor-densities of weight $+\frac{1}{2}$ being wholly determined and only these vectordensities playing a role, the idea of Weyl of replacing the potential vector by pseudovectors of class +1 and -1 seems much better." ([306], p. 261, footnote 19)

Schouten wrote down Dirac's equation in a space with torsion; his iterated wave equation, besides the mass term, contains a contribution $\sim-\frac{1}{4} R$ if torsion is set equal to zero. Whether Schouten could fully appreciate the importance of Weyl's new idea of gauging remains open. For him an important conclusion is that

"by the influence of a gravitational field the components of the potential vector change from ordinary numbers into Dirac-numbers." ([306], p. 265)

Two years later, Schrödinger as well became interested in Dirac's equation. We reproduce a remark from his publication [319]:

"The joining of Dirac's theory of the electron with general relativity has been undertaken repeatedly, such as by Wigner [419], Tetrode [344], Fock [131], Weyl [407, 408], Zaycoff [434], Podolsky [259]. Most authors introduce an orthogonal frame of axes at every event, and, relative to it, numerically specialised Dirac-matrices. This procedure makes it a little difficult to find out whether Einstein's idea concerning teleparallelism, to which [authors] sometimes refer, really plays a role, or whether there is no dependence on it. To me, a fundamental advantage seems to be that the entire formalism can be built up by pure operator calculus, without consideration of the $\psi$-function." 278 ([319], p. 105)

The $\gamma$-matrices were taken by Schrödinger such that their covariant derivative vanished, i.e., $\gamma_{l \| m}=\frac{\partial \gamma_{l}}{\partial x^{m}}-\Gamma_{l m}^{r}(g) \gamma_{r}+\gamma_{l} \Gamma_{m}-\gamma_{m} \Gamma_{l}=0$, where $\Gamma_{l}$ is the spin-connection introduced by $\psi_{\| l}=\frac{\partial \psi}{\partial x^{l}}-\Gamma_{l} \psi$. Schrödinger took $\gamma_{0}, \gamma^{i}$, with $i=1,2,3$, as Hermitian matrices. He introduced tensor-operators $T_{l m}^{i k}$ such that the inner product $\psi^{*} \gamma_{0} T_{l m}^{i k} \psi$ instead of $\psi^{*} T_{l m}^{i k} \psi$ stayed real under a "complemented point-substitution".

In the course of his calculations, Schrödinger obtained the wave equation

$$
\frac{1}{\sqrt{g}} \nabla_{k} \sqrt{g} g^{k l} \nabla_{l}-\frac{R}{4}-\frac{1}{2} f_{k l} s^{k l}=\mu^{2}
$$

where $\mu=2 \pi m c / h, f_{k l}$ is the electromagnetic field tensor, and $s^{k l}:=\frac{1}{2} \gamma^{[k} \gamma^{l]}$ with the $\gamma$-matrices $\gamma^{k}$, i.e., the spin tensor. As to the term with the curvature scalar $R$, Schrödinger was startled:

\footnotetext{
${ }^{277}$ Pseudo-tensors of class $k$ are defined by help of an auxiliary variable $\xi^{0}$ transforming like $\xi^{0^{\prime}}=\sigma \xi^{0}$; in their transformation law the multiplicative factor $\sigma^{k}$ occurs. $\sigma$ is a function of the coordinates involved in the tensor transformation law.

278 "Die Vereinigung der Diracschen Theorie des Elektrons mit der allgemeinen Relativitätstheorie ist schon wiederholt in Angriff genommen worden, [...]. Die meisten Autoren führen in jedem Weltpunkt ein orthogonales Achsenkreuz und in bezug auf dieses numerisch spezialisierte Diracsche Matrizen ein. Bei diesem Verfahren ist es ein bisschen schwer, zu erkennen, ob die Einsteinsche Idee des Fernparallelismus, auf die teilweise Bezug genommen wird, wirklich hereinspielt oder ob man davon unabhängig ist [...]. Ein grundsätzlicher Vorzug scheint es mir, dass sich der ganze Apparat fast vollständig durch reinen Operatorenkalkül aufbauen lässt, ohne auf die $\psi$-Funktion Bezug zu nehmen."
} 
"To me, the second term seems to be of considerable theoretical interest. To be sure, it is much too small by many powers of ten in order to replace, say, the term on the r.h.s. For $\mu$ is the reciprocal Compton length, about $10^{11} \mathrm{~cm}^{-1}$. Yet it appears important that in the generalised theory a term is encountered at all which is equivalent to the enigmatic mass term." 279 ([319], p. 128)

The coefficient $-\frac{1}{4}$ in front of the Ricci scalar in Schrödinger's (Klein-Gordon) wave equation differs from the $\frac{1}{6}$ needed for a conformally invariant version of the scalar wave equation ${ }^{280}$ (cf. [257], p. 395).

Bargmann in his approach, unlike Schrödinger, did not couple "point-substitutions [linear coordinate transformations] and similarity transformations [in spin space]" [6]. He introduced a matrix $\alpha$ with $\alpha+\alpha^{\dagger}=0$ such that $\left(\alpha \gamma^{l}\right)^{\dagger}=\left(\alpha \gamma^{l}\right)$, with $l=0, \ldots, 3$.

Levi-Civita wrote a letter to Schrödinger in the form of a scientific paper, excerpts of which became published by the Berlin Academy:

"Your fundamental memoir induced me to develop the calculational details for obtaining, from Dirac's equations in a general gravitational field, the modified form of your four equations of second order and thus make certain the corresponding additional terms. These additional terms do depend in an essential way on the choice of the orthogonal tetrad in the space-time manifold: It seems that without such a tetrad one cannot obtain Dirac's equation." 281 [208]

The last, erroneous, sentence must have made Pauli irate. In this paper, he pronounced his anathema (in a letter to Ehrenfest with the appeal "Please, copy and distribute!"):

"The heap of corpses, behind which quite a lot of bums look for cover, has got an increment. Beware of the paper by Levi-Civita: Dirac- and Schrödinger-type equations, in the Berlin Reports 1933. Everybody should be kept from reading this paper, or from even trying to understand it. Moreover, all articles referred to on p. 241 of this paper belong to the heap of corpses." 282 ([252], p. 170)

Pauli really must have been enraged: Among the publications banned by him is also Weyl's wellknown article on the electron and gravitation of 1929 [407].

Schrödinger's paper was criticised by Infeld and van der Waerden on the ground that his calculational apparatus was unnecessarily complicated. They promised to do better and referred to a paper of Schouten's [306]:

"In the end, Schouten arrives at almost the same formalism developed in this paper; only that he uses without need $n$-bein components and theorems on sedenions ${ }^{283}$,

\footnotetext{
279 "Das zweite Glied scheint mir von erheblichem theoretischen Interesse. Es ist freilich um viele Zehnerpotenzen zu klein, um etwa das Glied rechter Hand ersetzen zu können. Denn $\mu$ ist die reziproke Compton-Wellenlänge, ungefähr $10^{11} \mathrm{~cm}^{-1}$. Immerhin scheint es bedeutungsvoll, dass in der verallgemeinerten Theorie überhaupt ein mit dem rätselhaften Massenglied gleichartiges angetroffen wird."

${ }^{280}$ For arbitrary dimension $D \geq 2$ of the manifold $M_{D}$, the coefficient in the conformally invariant equation is $\frac{D-2}{4(D-1)}$.

281 "Ihre grundlegende Abhandlung hat mich veranlasst, die Rechnungseinzelheiten zu entwickeln, um von den Diracschen Gleichungen im allgemeinen Schwerefeld die modifizierte Form ihrer vier Gleichungen zweiter Ordnung zu gewinnen und so die entsprechenden Zusatzglieder festzustellen. Diese Zusatzglieder hängen in wesentlicher Weise von der Wahl des orthogonalen Vierbeines der Raumzeitmannigfaltigkeit ab: ein solches Bein scheint unentbehrlich zu sein, um die Diracschen Gleichungen zu bekommen."

282 "Der Leichenberg, hinter dem allerlei Gesindel Deckung sucht, hat einen Zuwachs erfahren. Es wird gewarnt vor der Arbeit von Levi-Civita: Diracsche und Schrödingersche Gleichungen, Berliner Berichte 1933. Alle sollten abgehalten werden, diese Arbeit zu lesen oder gar zu versuchen, sie zu verstehen. Ferner gehören sämtliche auf S. 241 dieser Arbeit zitierten Arbeiten dem Leichenberge an."

${ }^{283}$ Sedenions or quadri-quaternions form a system of 16 elements of an associative algebra; they are formed by the unit element 1 , four elements $E_{i}$ with $E_{i} E_{i}=1 ; E_{i} E_{j}=-E_{j} E_{i}$ and their products.
} 
while afterwards the formalism is still burdened with auxiliary variables and pseudoquantities. We have taken over the introduction of 'spin densities' by Schouten." 284 ([168], p. 4)

Unlike Schrödinger's, the wave equation derived from Dirac's equation by Infeld and Waerden contained a term $+\frac{1}{4} R$, with $R$ the Ricci scalar.

It is left to an in-depth investigation, how this discussion concerning teleparallelism and Dirac's equation involving Tetrode, Wigner, Fock, Pauli, London, Schrödinger, Infeld and van der Waerden, Zaycoff, and many others influenced the acceptance of the most important result, i.e., Weyl's transfer of the gauge idea from classical gravitational theory to quantum theory in 1929 [407, 408].

\subsubsection{Parallelism at a distance and electron spin}

Einstein's papers on distant parallelism had a strong but shortlived impact on theoretical physicists, in particular in connection with the discussion of Dirac's equation for the electron,

$$
\left(i \gamma^{k} \frac{\partial}{\partial x^{k}}+\mu\right) \psi=0
$$

where the 4-spinor $\psi$ and the $\gamma$-matrices are used. At the time, there existed some hope that a unified field theory for gravitation, electromagnetism, and the "electron field" was in reach. This may have been caused by a poor understanding of the new quantum theory in Schrödinger's version: The new complex wave function obeying Schrödinger's, and, more interestingly for relativists, Dirac's equation or the ensuing Klein-Gordon wave equation, was interpreted in the spirit of de Broglie's "onde pilote", i.e., as a classical matter wave, not - as it should have been - as a probability amplitude for an ensemble of indistinguishable electrons. One of the essential features of quantum mechanics, the non-commutativity of conjugated observables like position and momentum, nowhere entered the approaches aiming at a geometrization of wave mechanics.

Einstein was one of those clinging to the picture of the wave function as a real phenomenon in space-time. Although he knew well that already for two particles the wave function no longer "lived" in space-time but in 7-dimensional configuration space, he tried to escape its statistical interpretation. On 5 May 1927, Einstein presented a paper to the Academy of Sciences in Berlin with the title "Does Schrödinger's wave mechanics determine the motion of a system completely or only in the statistical sense?". It should have become a 4-page publication in the Sitzungsberichte. As he wrote to Max Born:

"Last week I presented a short paper to the Academy in which I showed that one can ascribe fully determined motions to Schrödinger's wave mechanics without any statistical interpretation. Will appear soon in Sitz.-Ber. [Reports of the Berlin Academy]." 285 ([103], p. 136)

However, he quickly must have found a flaw in his argumentation: He telephoned to stop the printing after less than a page had been typeset. He also wanted that, in the Academy's protocol, the announcement of this paper be erased. This did not happen; thus we know of his failed attempt, and we can read how his line of thought began ([183], pp. 134-135).

\footnotetext{
284 "Schouten kommt am Ende nahezu zum gleichen Formalismus, der in dieser Arbeit entwickelt wird; nur benutzt er zur Einführung dieses Formalismus unnötigerweise $n$-Beinkomponenten und Sätze über Sedenionen, während der Formalismus nachher noch durch Hilfsvariable und Pseudogrössen belastet wird. Die Einführung von 'Spinordichten' haben wir von Schouten übernommen."

285 "Vorige Woche habe ich der Akademie eine kleine Arbeit eingereicht, in der ich zeigte, dass man der Schrödinger Wellenmechanik ganz bestimmte Bewegungen zuordnen kann, ohne jede statistische Deutung. Erscheint baldigst in den Sitz.-Ber."
} 
Each month during 1929, papers appeared in which a link between Einstein's teleparallelism theory and quantum physics was foreseen. Thus, in February 1929, Wiener and Vallarta stressed that

"the quantities ${ }^{s} h_{\lambda}{ }^{286}$ of Einstein seem to have one foot in the macro-mechanical world formally described by Einstein's gravitational potentials and characterised by the index $\lambda$, and the other foot in a Minkowskian world of micro-mechanics characterised by the index $s$. That the micro-mechanical world of the electron is Minkowskian is shown by the theory of Dirac, in which the electron spin appears as a consequence of the fact that the world of the electron is not Euclidean, but Minkowskian. This seems to us the most important aspect of Einstein's recent work, and by far the most hopeful portent for a unification of the divergent theories of quanta and gravitational relativity." [418]

The correction of this misjudgement of Wiener and Vallarta by Fock and Ivanenko began only one month later [134], and was complete in the summer of 1929 [134, 133, 131, 132].

In March, Tamm tried to show

"that for the new field theory of Einstein [84, 88] certain quantum-mechanical features are characteristic, and that we may hope that the theory will enable one to seize the quantum laws of the microcosm." ${ }^{287}$ ([341], p. 288)

Tamm added a torsion term $i \hbar \sqrt{\left(S_{i} S^{i}\right)} \chi$ to the Dirac equation (197) and derived from it a general relativistic (Schrödinger) wave equation in an external electromagnetic field with a contribution from the spin tensor coupled to a torsion term ${ }^{288} \alpha^{[i} \alpha^{k]} S_{i k}{ }^{l}$. As Tamm assumed for the torsion vector $S_{k}= \pm \frac{i e}{\hbar c} \phi_{k}$, his tetrads had to be complex, with the imaginary part containing the electromagnetic 4-potential $\phi_{k}$. This induced him to see another link to quantum physics; by returning to the first of Einstein's field equations (170) and replacing $\epsilon$ in Equation (169) by $i \frac{e}{c} \hbar$ in the limit $\hbar \rightarrow 0$, he obtained the laws of electricity and gravitation, separately. From this he conjectured that, for finite $h$, Einstein's field equations might correctly reproduce the quantum features of "the microcosm" ([341], p. 291); cf. also [340].

What remained after all the attempts at geometrizing the matter field for the electron, was the conviction that the quantum mechanical "wave equations" could be brought into a covariant form, i.e., could be dealt with in the presence of a gravitational field, but that quantum mechanics, spin, and gravitation were independent subjects as seen from the goal of reaching unified field theory.

\subsubsection{Kaluza's theory and wave mechanics}

For some, Kaluza's introduction of a fifth, spacelike dimension seemed to provide a link to quantum theory in the form of wave mechanics. Although he did not appreciate Kaluza's approach, Reichenbächer listed various possibilities: With the fifth dimension, Kaluza and Klein had connected electrical charge, Fock the electromagnetic potential, and London the spin of the electron [276]. Also, the idea of relating Schrödinger's matter wave function with the new metrical component $g_{55}$ was put to work. Gonseth ${ }^{289}$ and Juvet, in the first of four consecutive notes submitted in August $1927[150,148,149,147]$ stated:

\footnotetext{
${ }^{286}$ The symbol ${ }^{s} h_{\lambda}$ for the tetrad field is due to Weitzenböck.

287 "dass für die neue Einsteinsche Feldtheorie gewisse quantenmechanische Züge charakteristisch sind und dass man hoffen darf, dass die Theorie die Erfassung der Quantengesetze des Mikrokosmos ermöglichen wird."

${ }^{288}$ For the alpha-matrices, cf. Equation (88).

289 Ferdinand Gonseth (1890-1975). Born in Sonvilier, Switzerland. Mathematician teaching first at the University of Bern and then at the Federal Institute of Technology (ETH) Zurich. His interest were in the foundations of mathematics, geometry and in problems of space and time. With G. Bachelard and P. Bernays he founded the philosophical review journal Dialectica.
} 
"The objective of this note is to formulate a five-dimensional relativity whose equations will give the laws for the gravitational field, the electromagnetic field, the laws of motion of a charged material point, and the wave equation of Mr. Schrödinger. Thus, we will have a frame in which to take the gravitational and electromagnetic laws, and in which it will be possible also for quantum theory to be included." 290 ([150], p. 543)

It turned out that from the $R_{55}$-component of the Einstein vacuum equations $R_{\alpha \beta}=0, \alpha, \beta=$ $1, \ldots, 5$, with the identification $g_{55}=\psi$ made, and the assumption that $\psi, \frac{\partial \psi}{\partial x^{i}}$ be "very small", while $\psi, \frac{\partial \psi}{\partial x^{5}}$ be "even smaller", the covariant d'Alembert equation followed, an equation that was identified by the authors with Schrödinger's equation. Their further comment is:

"We thus can see that the fiction of a five-dimensional universe provides a deep reason for Schrödinger's equation. Obviously, this artifice will be needed if some phenomenon would force the physicists to believe in a variability of the [electric] charge." ${ }^{291}$ ([149], p. 450)

In the last note, with the changed identification $g_{55}=\psi^{2}$ and slightly altered weakness assumptions, Gonseth and Juvet gained the relativistic wave equation with a non-linear mass term.

Interestingly, a couple of months later, O. Klein had the same idea about a link between the $g_{55}$-component of the metric and the wave function for matter in the sense of de Broglie and Schrödinger. However, as he remarked, his hopes had been shattered [189]. Klein's papers were of import: Remember that Kaluza had identified the fifth component of momentum with electrical charge [181], and five years later, in his papers of 1926 [185, 184], Klein had set out to quantise charge. One of his arguments for the unmeasurability of the fifth dimension rested on Heisenberg's uncertainty relation for position and momentum applied to the fifth components. If the elementary charge of an electron has been measured precisely, then the fifth coordinate is as uncertain as can be. However, Klein's argument is fallacious: He had compactified the fifth dimension. Consequently, the variance of position could not become larger than the compactification length $l \sim 10^{-30}$, and the charge of the electron thus could not have the precise value it has. In another paper, Klein suggested the idea that the physical laws in space-time might be implied by equations in five-dimensional space when suitably averaged over the fifth variable. He tried to produce wavemechanical interference terms from this approach [187]. A little more than one year after his first paper on Kaluza's idea, in which he had hoped to gain some hold on quantum mechanics, Klein wrote:

"Particularly, I no longer think it to be possible to do justice to the deviations from the classical description of space and time necessitated by quantum theory through the introduction of a fifth dimension." ([189], p. 191, footnote)

At about the same time, W. Wilson of the University of London rederived the Schrödinger equation in the spirit of O. Klein and noted:

"Dr. H. T. Flint has drawn my attention to a recent paper by O. Klein [189] in which an extension to five dimensions similar to that given in the present paper is described. The corresponding part of the paper was written some time ago and without any knowledge of Klein's work [...]." ([420], p. 441)

\footnotetext{
290 "L'object de cette note est de formuler une relativité à cinq dimensions dont les équations fourniront les lois du champ gravifique, du champ électromagnétique, les lois du mouvement d'un point matériel chargé et l'équation des ondes de M. Schrödinger. Nous aurons ainsi un cadre dans lequel entreront les lois de la gravitation et de l'électromagnétisme, et où il sera possible de faire entrer aussi la théorie des quanta."

291 "On voit ainsi que la fiction d'un univers à cinq dimensions permet de donner une raison profonde à l'équation de Schrödinger. Il est clair que cet artifice deviendrait nécessaire si quelque phénomène obligeait les physiciens à croir à la variabilité de la charge."
} 
Even Eddington ventured into the fifth dimension in an attempt to reformulate Dirac's equation for more than one electron; he used matrix algebra extensively:

"The matrix theory leads to a very simple derivation of the first order wave equation, equivalent to Dirac's but expressed in symmetrical form. It leads also to a wave equation which we can identify as relating to a system containing electrons with opposite spin. [...] It is interesting to note the way in which the existence of electrons with opposite spins locks the 'fifth dimension,' so that it cannot come into play and introduce the absolute into a world of relation. The domain of either electron alone might be rotated in a fifth dimension and we could not observe any difference." ([61], pp. 524, 542)

Eddington's "pentads" built up from sedenions later were generalised by Schouten [307].

J. W. Fisher of King's College re-interpreted Kaluza-Klein theory as presented in Klein's third paper [187]. He proceeded from the special relativistic homogeneous wave equation in fivedimensional space and, after dimensional reduction, compared it to the Klein-Gordon equation for a charged particle. By making a choice different from Klein's for a constant he rederived the result of de Broglie and others that null geodesics in five-dimensional space generate the geodesics of massive and massless particles in space-time [127].

Mandel of Petersburg/Leningrad believed that

"a consideration in five dimensions has proven to be well suited for the geometrical interpretation of macroscopic electrodynamics." ([221], p. 567)

He now posed the question whether this would be the same for Dirac's theory. Seemingly, he also believed that a tensorial formulation of Dirac's equation was handy for answering this question and availed himself of "the tensorial form given by W. Gordon [151], and by J. Frenkel" 292 [140]. Mandel used, in five-dimensional space, the complex-valued tensorial wave function $\Psi^{i k}=\psi \gamma^{i k}+$ $\Psi^{[i k]}$ with a 5 -scalar $\psi$. Here, he had taken up a suggestion J. Frenkel had developed during his attempt to describe the "rotating electron," i.e., Frenkel's introduction of a skew-symmetric wave function proportional to the "tensor of magneto-electric moment" $m_{i k}$ of the electron by $m_{i k} \psi=m_{0} \psi_{i k}[141,140]$. $\Psi^{i k}$ may depend on $x^{5}$; by taking $\Psi$ periodic in $x^{5}$, Mandel derived a wave equation "which can be understood as a generalisation of the Klein-Fock five-dimensional wave equation [...]." He also claimed that the vanishing of $\psi$ made $M_{5}$ cylindrical (in the sense of Equation (109) [221]). As he had taken notice of a paper of Jordan [173] that spoke of the electromagnetic field as describing a probability amplitude for polarised photons, Mandel concluded that the amplitude of his $\Psi$-field might then represent polarised electrons as its quanta. However, he restricted himself to the consideration of classical one-particle wave equations because

"in some cases one can properly speak of a quasi-macroscopical one-body problem -

think of a beam of monochromatic cathod-rays in an arbitrary external force-field." 293

In a later paper, Mandel came back to his wave equation with a skew-symmetric part and gave it a different interpretation [222].

Unlike Klein, Mandel tried to interpret the wave function as a new discrete coordinate, an idea going back to Pauli [247]. He took "Dirac's spin variable" $\zeta$ and the spatial coordinate $x^{5}$ as a pair of canonically-conjugate operator-valued variables; $\zeta$ is linked to positive and negative elementary charge (of proton and electron) as its eigenvalues. In Mandel's five-dimensional space, the fifth coordinate, as a "charge" coordinate, thus assumed only 2 discrete values $\pm e$.

\footnotetext{
${ }^{292}$ Gordon has not given a tensorial form of Dirac's equation but his well-known decomposition of the Dirac current into a conduction and a polarisation part.

293 "in manchen Fällen kann wohl eigentlich von einer quasi-makroskopischen Betrachtung des Einkörperproblems die Rede sein, man denke etwa an ein Bündel von monochromatischen Kathodenstrahlen."
} 
"This completely corresponds to the procedure of the Dirac theory, with the only difference that for Dirac the coordinate $\zeta$ could assume not 2 but 4 values; from our point of view this remains unintelligible." ${ }^{294}$ ([222], p. 785)

In following Klein, Mandel concluded from the Heisenberg uncertainty relations that

"[...] all possible values of this quantity $\left[x^{5}\right]$ still remain completely undetermined such that all its possible values from - inf to + inf are of equal probability."

This made sense because, unlike Klein, Mandel had not compactified the fifth dimension. His understanding of quantum mechanics must have been limited, though: Only two pages later he claimed that the canonical commutation relations $[\mathbf{p}, \mathbf{q}]=\frac{\hbar}{i} \mathbf{1}$ could not be applied to his pair of variables due to the discrete spectrum of eigenvalues. He then essentially went over to the Weyl form of the operators $p, q$ in order to "save" his argument [222].

Another one of the many versions of "Dirac's equation" was presented, in December 1930, by Zaycoff who worked both in the framework of Einstein's teleparallel theory and of Kaluza's five-dimensional space. His Lagrangian is complicated ${ }^{295}$,

$$
M=-i \tilde{\psi} \gamma^{\rho} \frac{\partial \psi}{\partial x^{\rho}}+\frac{i}{2} S_{m} J_{m}+\frac{1}{24} S_{k l m} J_{k l m}+a f_{m} J_{m}+\frac{1}{8} F_{k m} J_{k m}+\mu J_{0}
$$

where summation is implied and $S_{k l}^{m}$ is the torsion tensor, $f_{m}$ the electromagnetic vector potential, and $f_{i k}$ the electromagnetic field. Note that the Dirac current $J_{m}:=\tilde{\psi} \gamma_{m} \psi$ couples to both the torsion vector and the 4-potential. The remaining variables in (198) are $J_{m l}:=i \tilde{\psi} \gamma_{m} \gamma_{l}^{\dagger} \gamma_{0} \psi$, with $k \neq l$, and $J_{k l m}:=i \tilde{\psi} \gamma_{k} \gamma_{l}^{\dagger} \gamma_{m} \psi$, with $k \neq l \neq m$ [434].

While Zaycoff submitted his paper, Schouten lectured at the MIT. and, among other things, showed "how the mass-term in the Dirac equations comes in automatically if we start with a five-dimensional instead of a four-dimensional Riemannian manifold" ([306], p. 272). He proved a theorem:

The Dirac equations for Riemannian space-time with electromagnetic field and mass can be written in the form of equations without field or mass $\alpha^{b} \nabla_{b} \psi=0$ in an $R_{5}$.

Here $\alpha^{b}$ is the set of Dirac numbers defined by $\alpha^{(a} \alpha^{b)}=g^{a b},\left(\alpha^{a} \alpha^{b}\right) \alpha^{c}=\alpha^{a}\left(\alpha^{b} \alpha^{c}\right)$ with $a, b, c=$ $0, \ldots, 4$, and $\nabla_{b}$ the covariant spinor derivative defined by him.

As we mentioned above (cf. Section 6.3.2), another approach to the matter within projective geometry was taken by Pauli with his student J. Solomon [253]. After these two joint publications, marred by a calculational error, Pauli himself laid out his version of the projective theory in two installments with the first, as a service to the community, being a pedagogical presentation of the formalism connected with projective geometry [249]. The second paper, again, has the application to Dirac's equation as a prime motivation:

"The following deductions are intended to show [...] that the unifying combination of the gravitational and the electromagnetic fields, by projective differential geometry with the aid of five homogeneous coordinates, is a general method whose range reaches beyond classical field-physics and into quantum theory. Perhaps, the hope is not unjustified that the method will stand the test as a general framework for the laws of physics

\footnotetext{
294 "Das entspricht vollständig dem Verfahren der Diracschen Theorie, nur mit dem Unterschied, dass bei Dirac die Koordinate $\xi$ nicht zwei, sondern vier Werte haben konnte, was von unserem Standpunkt aus unverständlich bleibt."

${ }^{295}$ Although Zaycoff does not say it, he takes $\tilde{\psi}$ as the complex-conjugate transposed function.
} 
also with regard to a future physical and conceptual improvement of the foundations of Dirac's theory." 296 ([250], pp. 837-838)

Pauli started with the observation that the group of orthogonal transformations in five-dimensional space had an irreducible, four-dimensional matrix representation satisfying

$$
\alpha_{\mu} \alpha_{\nu}+\alpha_{\nu} \alpha_{\mu}=2 g_{\mu \nu} \cdot 1, \quad \mu, \nu=1, \ldots, 5,
$$

where $\alpha_{\mu}$ are $4 \times 4$ matrices given at the end of Section 2.1.5 in a different representation $\alpha_{\mu}=$ $\left(\begin{array}{cc}\sigma_{\mu} & \mathbf{0} \\ \mathbf{0} & -\sigma_{\mu}\end{array}\right)$ with $\mu=1,2,3, \alpha_{4}=\left(\begin{array}{ll}\mathbf{1} & \mathbf{0} \\ \mathbf{0} & \mathbf{1}\end{array}\right)$, and augmented by $\alpha_{5}=\left(\begin{array}{ll}\mathbf{0} & \mathbf{1} \\ \mathbf{1} & \mathbf{0}\end{array}\right)$. This had been known also to Eddington [61] and Schouten [303]. He then introduced projective spinors depending on five homogeneous coordinates without using bein-quantities. He followed the methods of Schrödinger and Bargmann [319, 6], i.e., used the existence of a matrix A such that $\mathbf{A} \alpha_{\mu}$ is Hermitian. The transformation laws of 4 -spinors $\Psi$ and matrices $\alpha_{\mu}$ are coupled:

$$
\begin{aligned}
\psi^{\prime} & =S^{-1} \Psi, \\
\alpha_{\mu}^{\prime} & =S^{-1} \alpha_{\mu} S, \\
A^{\prime} & =S^{\dagger} A S .
\end{aligned}
$$

For transformations in the space of homogeneous coordinates $X^{\prime \mu}=a^{\mu}{ }_{\nu} X^{\nu}$ such that $\alpha_{\nu}=a^{\mu}{ }_{\nu} \alpha_{\mu}^{\prime}$, the quantity $a_{\mu}:=\Psi^{\star} A \alpha_{\mu} \Psi$ transforms, for fixed $\alpha_{\mu}$, under changes of the spin frame, Equation (200), as a covariant vector ${ }^{297}$.

Pauli criticised an analogous attempt at formulating Dirac's equation with the help of five homogeneous coordinates by Schouten and van Dantzig [317, 308, 309] as being "difficult to understand and less than transparent" ${ }^{298}$. A projective spinor is defined via

$$
\Psi=\psi F^{l},
$$

where $\psi$ is a normal ("affine") spinor (degree of homogeneity 0) and $F$ a real scalar of (homogeneity) degree 1, i.e., $F=X^{\mu} \frac{\partial F}{\partial X^{\mu}}$. There exist two (related) spin-connections $\Lambda_{k}$ for projective spinors $\Psi$ and $\stackrel{\mathrm{R}}{\Lambda}_{k}$ for spinors $\psi$.

Pauli's Dirac equation, derived from a Lagrangian, looked in five dimensions like

$$
\alpha^{\mu}\left(\Psi_{; \mu}+k X_{\mu} \Psi\right)=0
$$

with $k=-\frac{i m c}{h}-\frac{i e}{h c} \frac{c}{\sqrt{\kappa}} \frac{1}{r}$, and $l=+\frac{i e}{h c} \frac{c}{\sqrt{\kappa}} \frac{1}{r}$. The covariant derivative is formed with the spin connection $\Lambda_{k}$. An involved calculation leads to Dirac's equation in four dimensions:

$$
\alpha^{k}\left(\frac{\partial \psi}{\partial x^{k}}+\stackrel{\mathrm{R}}{\Lambda}_{k} \psi-\frac{i e}{h c} \Phi_{k} \psi\right)-i \frac{m c}{h} \alpha_{0} \psi+\frac{r}{8} \frac{\sqrt{\kappa}}{c} F_{k l} \alpha_{0} \alpha^{[k l]} \psi=0,
$$

with the numerical factor $r$, the electrical 4-potential $\Phi_{k}$, and the electromagnetic field tensor $F_{k l}$; furthermore, $\alpha^{\mu \nu}=\alpha^{[\mu} \alpha^{\nu]}$.

Pauli succeeded also in formulating a five-dimensional energy-momentum tensor containing, besides the four-dimensional energy-momentum tensor, the four-dimensional Dirac current vector. At the end of his paper Pauli stressed the

296 "Die folgenden Ausführungen sollen [...] zeigen, dass die einheitliche Zusammenfassung des Gravitations- und des elektromagnetischen Feldes durch die projektive Differentialgeometrie mittels fünf homogener Koordinaten eine allgemeine Methode ist, deren Tragweite über die klassische Feldphysik hinaus- und in die Quantentheorie hineinreicht. Vielleicht ist es nicht unberechtigt, zu hoffen, dass die Methode als allgemeiner Rahmen der physikalischen Gesetze sich auch gegenüber einer künftigen physikalisch-begrifflichen Verbesserung der Grundlagen der Diracschen Theorie bewähren wird."

${ }^{297} \Psi^{\star}$ is the complex conjugate and transposed object.

298 "schwer verständlich und unübersichtlich" 
"more provisional character of his 5-dimensional-projective form of Dirac's theory. [...] In contrast to the joinder of the gravitational and electromagnetic fields, a direct logical coupling of the matter-wave-field with these has not been achieved in the form of the theory developed here."

\subsection{Einstein, spinors, and semi-vectors}

Ehrenfest, even after van der Waerden's paper on spinor analysis [368], in 1932 pressed Einstein to think about a simple geometric interpretation of spinors. To this end Einstein responded, together with his assistant Mayer, by introducing the concept of semi-vector seemingly more natural to him than a spinor, and also more general:

"In spite of the great importance which the spinor concept, as introduced by $\mathrm{P}$ a u li and D i r a c, has obtained in molecular physics, one cannot claim that the analysis of this concept up to now satisfies all justified demands. Our efforts have lead to a derivation corresponding, according to our opinion, to all demands for clarity and naturalness and avoiding completely any not so transparent artifice. Thereby, [...] the introduction of novel quantities was shown to be necessary, the 'semi-vectors', which include spinors but possess a clearly more transparent transformation character than spinors." 299 ([110], p. 522)

In this first publication on the subject, Einstein and Mayer explicitly referred to the paper by Infeld and van der Waerden, of which they had received a copy several months before publication ([167], and [110], p. 25, footnote). Apparently, Einstein found the reconstruction of the spinor concept in his paper more "clear and natural" than Infeld and van der Waerden's. Nevertheless, the approach and notation of Infeld and van der Waerden became the accepted one by physicists.

About three months before the first paper on semi-vectors was published, Einstein wrote to Besso:

"I work with my Dr. Mayer on the theory of spinors. We already could clear up the mathematical relations. A grasp on the physics is far away, farther than one thinks at present. In particular, I still am convinced that the attempt at an essentially statistical theory will fail." ${ }^{300}$ ([99], p. 291)

Besides the aspired-to clarity and simplicity, Einstein's main hope was that, with his semivector system of equations replacing Dirac's equation, it might be possible to explain the existence of elementary particles with opposite charge and unlike mass, i.e., of electron and proton. As noted before, he had not been able to solve this problem by his affine field theory of 1923 (cf. Section 4.3.2), nor by the approaches to unified field theory that followed. As it turned out, the positron was discovered at about the same time, and the problem dissolved while Einstein and Mayer began to reformulate the spinor concept. Einstein again seems to have been fully convinced that his new concept of "semi-vector" was superior to the spinor concept. On 7 May 1933, he wrote to De Haas:

\footnotetext{
299 "Bei der grossen Bedeutung, welche der von P a u li und D i r a c eingeführte Spinor-Begriff in der Molekularphysik erlangt hat, kann doch nicht behauptet werden, dass die bisherige mathematische Analyse dieses Begriffs allen berechtigten Ansprüchen genüge. [...] Unsere Bemühungen haben zu einer Ableitung geführt, welche nach unserer Meinung allen Ansprüchen an Klarheit und Natürlichkeit entspricht und undurchsichtige Kunstgriffe völlig vermeidet. Dabei hat sich [...] die Einführung neuartiger Grössen, der 'Semi-Vektoren', als notwendig erwiesen, welche die Spinoren in sich begreifen, aber einen wesentlich durchsichtigeren Transformationscharakter besitzen als die Spinoren."

300 "Ich arbeite mit meinem Dr. Mayer an der Theorie der Spinoren. Wir haben die mathematischen Zusammenhänge schon klären können. Von einer Erfassung des Physikalischen ist man noch weit entfernt, viel weiter, als man gegenwärtig denkt. Besonders bin ich nach wie vor davon überzeugt, dass der Versuch einer wesentlich statistischen Theorie scheitern wird."
}

Living Reviews in Relativity

http: //www. livingreviews.org/lrr-2004-2 
"Scientifically Mayer and I have found one very natural generalisation of Dirac's equation which makes it comprehensible, that there are two understandable elementary masses, while there is only one electric charge." [69]

In the first paper in the reports of the Berlin Academy, the mathematical foundations of the semi-vector formalism are developed [110]. The basic idea of Einstein and Mayer is the possibility of a decomposition of any (proper) Lorentz transformation described by a real matrix $\mathbf{D}$ into a product $\mathbf{B C}$ of a pair of complex-conjugate, commuting matrices ${ }^{301} \mathbf{B}$ and $\mathbf{C}$. The transformations represented by $\mathbf{B}$ or $\mathbf{C}$ form a group isomorphic to the Lorentz group. In terms of infinitesimal Lorentz transformations given by an antisymmetric tensor $\omega_{i k}$, this amounts to the decomposition into a self-dual and an anti-selfdual part: $\omega_{i k}=\frac{1}{2}\left(\omega_{i k}+i \omega_{i k}^{*}\right)+\frac{1}{2}\left(\omega_{i k}-i \omega_{i k}^{*}\right)$, with the dual ${ }^{302}$ defined by $\omega_{i k}^{*}:=\frac{1}{2} \sqrt{g} \epsilon_{i j k l} \omega^{k l}$.

Contravariant semi-vectors of the first and second kind now are defined by their transformation laws: $\rho^{i^{\prime}}=b^{i}{ }_{k} \rho^{k}$ and $\sigma^{i^{\prime}}=c^{i}{ }_{k} \sigma^{k}$, where $b^{i}{ }_{k}, c^{i}{ }_{k}$ are the components of $\mathbf{B}$, C. For real Lorentz transformation $\mathbf{D}, \bar{b}^{i}{ }_{k}=c^{i}{ }_{k}$ must hold. As $\mathbf{B}, \mathbf{C}$ are both also Lorentz transformations,

"the metric tensor $g_{i k}$ is also a semi-vector of 1 st kind (and of 2 nd kind) with transformationinvariant components."

Thus it can be used for raising and lowering indices of semi-vectors ([110], p. 535).

The system of equations intended as a replacement of the Dirac equation appears only in the second publication [111]. A Lagrange function for the semi-vector is found and the generalised Dirac equations for the semi-vectors $\psi, \chi$ look like ${ }^{303}$ :

$$
E^{r \sigma \tau}\left(\frac{\partial \psi_{\sigma}}{\partial x^{r}}-i \epsilon \psi_{\sigma} \phi_{r}\right)=\bar{C}^{\tau \rho} \chi_{\rho}, \quad E^{\star r \sigma \tau}\left(\frac{\partial \chi_{\tau}}{\partial x^{r}}-i \epsilon \chi_{\tau} \phi_{r}\right)=-C^{\rho \sigma} \psi_{\rho}
$$

where $\phi_{r}$ is the electromagnetic 4-potential and $E^{r \sigma \tau}$ a numerically invariant tensor depending on 4 constants $a_{(r)}$ :

$$
E_{r s t}=g_{r s} a_{(t)}+g_{r t} a_{(s)}-g_{s t} a_{(r)}-\sqrt{g} \eta_{r s t w} a^{(w)} ; \quad a^{(w)}=g^{w t} a_{(t)} .
$$

Contrary to his idea of what a "real" unified field theory should look like, Einstein just added the Lagrangian for the semi-vector fields to the Lagrangians for the gravitational and electromagnetic fields.

In his "Spencer Lecture" of 10 June 1933 in Oxford, Einstein embedded his point of view into the development of field theory:

"[... Louis de Broglie guessed the existence of a wave field that could be used for the interpretation of certain quantum properties of matter. With the spinors, Dirac found novel field quantities whose simplest equations permitted the derivation of the properties of the electron to a great extent. With my collaborator, Dr. Walther Mayer, I now found that these spinors form a special case of a type of field, linked to four-dimensional space, which we called 'semi-vectors'. The simplest equations to be satisfied by such semi-vectors provide a key for the understanding of the existence of two elementary particles with different ponderable mass and like, but opposite, charge. These semivectors are, besides the usual vectors, the simplest mathematical field-objects possible

\footnotetext{
${ }^{301}$ Two matrices $\mathbf{B}$ and $\mathbf{C}$ are called commuting if $[\mathbf{B}, \mathbf{C}]:=\mathbf{B C}-\mathbf{C B}=0$.

${ }^{302} \epsilon_{i j k l}$, the components of which are either \pm 1 or 0 , is the totally antisymmetric (Levi-Civita) tensor density keeping its components fixed under any coordinate transformation (cf. Section 2.1.5).

${ }^{303}$ All indices run from 1 to 4 .
} 
in a four-dimensional metrical continuum; it appears that they naturally describe essential properties of the electrical elementary particles." 304 ([100], p. 117)

That semi-vectors are in close connection with spinors and not always the simplest objects, had been noted by Schouten in a paper submitted to Zeitschrift für Physik on 15 April 1933, even before Einstein's Spencer lecture:

"The space of semivectors of first and second kind is the manifold of two simple bivectors in the local space-time-world, which lie on the null cone in two planes of the first and second system of planes, respectively. [...] In the not-projective theory as well as in the projective without an electromagnetic field, semi-vectors have an advantage, [...]. As soon as an electromagnetic field is present, in the projective theory, calculation with spinvectors is simpler than calculation with semivectors." ${ }^{305}$ [309]

In a previous paper, Schouten had geometrically explained the two approaches to spinor analysis followed by van der Waerden [368], Laporte and Uhlenbeck [203], and by himself. In the first approach, the vectors of the two invariant planes in spin space were identified; in the second, i.e., in Schouten's, they were taken as the basis for a four-dimensional vector space [308]. Schouten had been corresponding with Pauli who "was so friendly as to allow me to quote this theorem from a not yet published manuscript." ${ }^{306}$ In his second paper, Schouten placed the semi-vectors of Einstein and Mayer into his geometrical setting. He showed that there exist two different complexconjugated three-dimensional representations of the real Lorentz group as a 6-parameter subgroup of the four-dimensional orthogonal group. With the help of these he constructed four

"building blocks: the special spin vectors of 1st and 2nd kind of spin space, which at the same time are special semi-vectors in the two preferred invariant planes in semi-space. In both cases, always two vectors are joined which belong to different invariant planes, for semi-vectors two with same transformation law, for spin vectors two with conjugate complex transformations." ([309], p. 106)

As Schouten used group theory, quaternions, sedenions, projective geometry - all not very familiar to physicists - Pauli must have thought of a much simpler disentanglement of semi-vectors and spinors. In fact, as Pauli pointed out in his letter to Einstein of 16 July 1933, a semi-vector does not form an irreducible representation of the rotation group while a spinor does ${ }^{307}$ (see [252], p. 189). In this regard, the semivector concept is less "natural" than the spinor concept. In contrast to what Schouten had shown, in his book on spinors Cartan flatly disclaimed any

\footnotetext{
304 “.... Louis de Broglie erriet die Existenz eines Wellenfeldes, das zur Deutung von gewissen Quanteneigenschaften der Materie verwendbar war. Dirac fand in den Spinoren neuartige Feldgrössen, deren einfachste Gleichungen die Eigenschaften des Elektrons weitgehend abzuleiten gestatten. Ich fand nun mit meinem Mitarbeiter, Dr. Walther Mayer, dass diese Spinoren einen Spezialfall einer, mathematisch mit dem Vierdimensionalen verknüpften Feldart bilden, die wir als 'Semivektoren' bezeichneten. Die einfachsten Gleichungen, welchen solche Semivektoren unterworfen werden können, geben einen Schlüssel für das Verständnis der Existenz von zweierlei Elementarteilchen verschiedener ponderabler Masse und gleicher, aber entgegengesetzter Ladung. Diese Semivektoren sind nach den gewöhnlichen Vektoren die einfachsten mathematischen Feldgebilde, die in einem metrischen Kontinuum von vier Dimensionen möglich sind, und es scheint, dass sie zwanglos wesentliche Eigenschaften der elektrischen Elementarteilchen beschreiben."

305 "Der Raum der Semivektoren erster bzw. zweiter Art ist die Mannigfaltigkeit der Systeme von zwei einfachen Bivektoren der lokalen Raumzeitwelt, die in zwei Ebenen des ersten bzw. zweiten Ebenensystems auf dem Nullkegel liegen. [...] In der nicht projektiven Theorie sowie in der projektiven bei fehlendem elektromagnetichen Feld haben die Semivektoren einen Vorzug, [...]. Sobald in der projektiven Theorie ein elektromagnetisches Feld auftritt, ist die Rechnung mit Spinvektoren einfacher als die mit Semivektoren."

${ }^{306}$ Schouten referred to Pauli several times ([308], pp. 406, 414, 416-417).

${ }^{307} \mathrm{In}$ his letter to Einstein, Pauli had also mentioned his papers to be published in Annalen der Physik and discussed in Section 7.2.3.
} 
geometric definition for semivectors ${ }^{308}$. Pauli then set his doctoral student V. Bargmann on the problem of comparing Einstein's semivector approach to the spinor calculus as developed by van der Waerden. Bargmann acknowledged Schouten's paper [309], "with which our presentation has quite a few points of contact". He then proved that

"to each semi-vector two 2-component spinors correspond, which both satisfy the same transformation law." ([7], p. 68)

and that the generalised Dirac equations of Einstein and Mayer (206)

"decompose into two separate 4-component systems of Dirac's type, which are distinguished only by the mass values." ([7], p. 78)

This means that only one of these two systems is needed, and that it describes particles with opposite charge and the same mass. Thus, as van Dongen states curtly:

"It is evident from Bargmann's analysis that the most general semi-vector Dirac system of Einstein and Mayer is nothing more than just a linear superposition of two independent Dirac spinor systems and thus cannot give insight into the fundamental nature of electrons and protons." ([371], p. 88, and [372])

In his letter to Einstein, Pauli had also mentioned his papers to be published in Annalen der Physik and discussed here in Section 7.2.3. Two more papers were written by Einstein and Mayer before Einstein quietly dropped the subject. The last paper considered semi-vectors as "usual vectors with a different differentiation character" $[112,113]$.

It seems that Einstein at the time had not followed quantum field theory intensively enough to be able to compete with that theory - leaving aside his rejection of "the statistical fad". By continuing to pair "electron and proton" while others speculated already about "electron and its antiparticle" 309 or "proton and its antiparticle", he was bound to run into a dead end. About one year after the Spencer lecture, when Einstein was still publishing about semi-vectors, Pauli and Weisskopf quantised the scalar relativistic wave equation with an external field using Bose-Einstein statistics:

"Without further hypothesis the existence follows of particles with opposite charge and same rest mass, which can be produced or annihilated by absorption or emission of electromagnetic radiation. The frequency of these processes is shown to be of the same magnitude as the one for particles with the same charge and mass following from Dirac's hole theory." 310 ([255], Abstract)

Of course, Einstein's problem was quickly solved; at about the same time the electron's antiparticle was observed.

\footnotetext{
${ }^{308}$ This reference to Cartan is taken from van Dongen's dissertation ([371], p. 86, footnote 70). After all, Cartan had discovered spinors in 1913 in the context of his classification of simple Lie algebras [370].

${ }^{309}$ Dirac had made an antiparticle to the electron appear in his theory as early as in 1930 [55].

310 "Dabei ergibt sich ohne weitere Hypothese die Existenz von zueinander entgegengesetzt geladenen Teilchen gleicher Ruhmasse, die unter Absorption bzw. Emission von elektromagnetischer Strahlung paarweise erzeugt bzw. vernichtet werden können. Die Häufigkeit solcher Prozesse erweist sich als von derselben Grössenordnung wie die für Teilchen derselben Ladung und Masse aus der Diracschen Löchertheorie folgende."
} 


\section{Less Than Unification}

During the time span considered here, there also were those who did not adhere to the program of unified field theory, or changed their mind about it. The mathematician G. D. Birkhoff belonged to the first group. Instead of removing the matter tensor in favour of some general geometry, he just remained in the old setting of Einstein's theory: To the energy-momentum tensors of a perfect fluid and of the electromagnetic field on the r.h.s. of Einstein's field equations, he added a contribution, named the "atomic potential tensor" describing a scalar wave function $\psi$ à la Schrödinger: $A_{i j}=\psi g_{i j}$ with the "potential" $\psi$. He proposed to apply this theory

"to the consideration of the small oscillations of the proton and single electron forming the hydrogen atom." [18, 17].

Another one was the Russian physicist G. Rumer, whose somewhat exotic suggestion, within a framework of Kantian philosophy, was to remain in Riemannian geometry and keep to Einstein's vacuum field equations but raise the number of the dimension of the underlying manifold. He named such a manifold an $F_{n}$ and considered a three-dimensional Riemannian subspace $V_{3}$ embedded, locally and isometrically, into $F_{n}$. Then, by use of the Gauss-Codazzi and Ricci-Codazzi equations, he decomposed the Ricci tensor of $F_{n}$ into its part in $V_{3}$ and the rest. His classification went as follows:

"We have seen that the $V_{3}$ itself is either an $F_{3}$ (in this case it is empty), or a subspace of an $F_{4}$ (thus it contains a gravitational field), or a subspace of $F_{5}$ (then also an electrical field is present). In the case in which 'something' exists which is neither a gravitational nor an electrical field, the $V_{3}$ must be a subspace of $F_{n}(n \geq 6)$. However, geometry shows that every $V_{3}$ is a subspace of a particular $F_{6}$, i.e., the Euclidean or pseudo-Euclidean space. This shows us that transition to $F_{6}$ is the final step." 311 ([285], p. 277)

The unidentified parts in the decomposition of the Ricci tensor into its piece in $V_{3}$ and the rest Rumer ascribed to "matter." He acknowledged Born's

"[...] stimulation and his interest extended toward the completion of this paper." 312

Born himself was mildly skeptical ${ }^{313}$ :

"[...] a young Russian surfaced here who brought with him a 6-dimensional relativity theory. As I already felt frightened by the various 5-dimensional theories, and had little confidence that something beautiful would result in this way, I was very skeptical." 314

After Lanczos had (mildly) criticised Einstein's parallelism at a distance [201], he seemed to have lost confidence in Einstein's program for unification and became a "renegade". He developed a theory by which

\footnotetext{
311 "Wir haben gesehen dass ein $V_{3}$ entweder selbst ein $F_{3}$ ist (dann ist er leer) oder ein Unterraum von $F_{4}$ (dann ist in ihm ein Gravitationsfeld vorhanden), oder ein Unterraum von $F_{5}$ (dann ist in ihm auch ein reines Elektrizitätsfeld vorhanden). Wo also noch 'etwas' ist, das weder Gravitationsfeld noch Elektrizitätsfeld ist, muss der $V_{3}$ ein Unterraum in $F_{n}(n \geq 6)$ sein. Nun zeigt aber die Geometrie, dass jeder $V_{3}$ ein Unterraum eines speziellen $F_{6}$, nämlich des euklidischen oder pseudo-euklidischen Raumens ist. Das zeigt uns, dass der Übergang zum $F_{6}$ auch der letzte Schritt ist."

312 "[...] Anregungen und das Interesse, das er dem Werden dieser Arbeit entgegenbrachte."

${ }^{313}$ Born tried to support Rumer in various ways, as can be seen from his correspondence with Einstein [103].

314 “...] tauchte hier ein junger Russe auf, der eine 6-dimensionale Relativitätsheorie mitbrachte. Da ich bereits vor den verschiedenen 5-dimensionalen Theorien Angstgefühle empfand und wenig Zuversicht, dass auf diesem Wege etwas Schönes herauskommen könnte, war ich sehr skeptisch."
} 
"[...] the basic properties of the electromagnetic field may be derived effortlessly from the general properties of Riemannian geometry by use of a variational principle characterised by a very natural demand." 315 ([202], p. 168)

For his Lagrangian, he took $\mathcal{L}=R_{i k} R^{i k}+C\left(R_{k}^{k}\right)^{2}$, with $C$ being a constant. He first varied with respect to the metric $g_{i k}$ and the Ricci tensor $R_{i k}$ as independent variables, and then expressed the variation $\delta R_{i k}$ with $\delta g_{i j}$. The resulting variation is then set equal to zero. In the process "spontaneously" a

"free vector appears for which, later, a restraining equation of the type of the equation for the [electromagnetic] potential results - as a consequence of the conservation laws for energy and momentum."

Also Rainich's approach [265] mentioned in Section 6.1, which, in the case of Maxwell's equations without sources, and for non-null electromagnetic fields, did substitute a set of algebraic conditions on the Einstein tensor for Maxwell's equations, might be seen as an alternative for the unification of gravity and electromagnetism. According to L. Witten:

"The only criterion for a unified field theory that these equations do not satisfy is that they are not derived from a variational principle by means of a Lagrange's function involving geometric quantities alone." ([422], p. 397)

Finally, van Dantzig's program after 1934, which we briefly met in Section 1, might be considered. It aimed at showing, eventually, that the

"metric should turn out finally to be a system of some statistical mean values of certain physical quantities." ([363], p. 522)

This meant turning upside down Einstein's geometrization program for matter.

\footnotetext{
315 “[...] aus den allgemeinen Eigenschaften der Riemannschen Geometrie, unter Zugrundelegung eines durch eine sehr natürliche Forderung ausgezeichneten Wirkungsprinzips, ohne jeden besonderen Kunstgriff die Grundeigenschaften des elektromagnetischen Feldes zwanglos abgeleitet werden können."
} 


\section{Mutual Influences Among Mathematicians and Physicists?}

A most interesting task far beyond this review would be to reconstruct, in detail, the mutual influences among researchers in the development of the various strands of unified field theory. An interesting in-depth-study for the case of Weyl has already been made [326].

It seems safe to say that the mathematical development of differential geometry in the direction of affine and metric-affine geometry received its original impetus from Einstein's general relativity and Weyl's extension of it (see statements by Hessenberg 1917, Schouten 1922, Cartan 1922). Although a mathematician, Weyl understood some of his work to be research in physics proper. In this, he was criticised by Pauli, who gave in only when Weyl shifted his gauge idea from coupling electromagnetism to gravitation to coupling electromagnetism to the quantum mechanical state function for an electron. Weyl's influence was prominent among both parties, mathematicians (Cartan, Schouten, Struik, Eisenhart, Hlavaty, Wiener, etc.) and physicists (Eddington, Einstein, Reichenbächer, Mandel, Fock, Zaycoff, et al). In the review on differential geometry by the mathematician Berwald ${ }^{316}$, Weyl plays a prominent role while Einstein is mentioned only in passing in connection with "Einstein-manifolds" (Einstein-spaces) ([13], p. 163). Berwald discusses Eddington's affine theory as the most modern development with, again, Einstein's papers [77, 74, 76] noted without comment. Astonishingly, while being more or less silent on Einstein, Berwald refers to the book on general relativity by von Laue [386] and to Pauli's article in the Encyclopedia of Mathematical Sciences [246]. He notes papers by Bach (alias Förster) [4] and G. Juvet [180] on Weyl's theory, and a paper of Kretschmann [196]. Unlike Berwald, Weitzenböck in his review article on the theory of invariants mentions Einstein directly:

"In recent years, due to their use in modern physical theories, the theory of differential forms (tensors) was elaborated extensively. We mention Ricci, Levi-Civita, Hessenberg, Einstein, Hilbert, [Felix] Klein, E. Noether, Weyl." 317 [392]

Einstein is mentioned as the only theoretical physicist among seven mathematicians. The development of projective geometry did profit from the mathematician Kaluza's idea of a five-dimensional space as the arena for unified field theory. It enticed physicists such as Pauli, O. Klein, Mandel, Fock, Infeld, and inspired mathematicians such as Veblen, Schouten, van Dantzig, Cartan, and others.

Of course, in terms of co-authorship and mutual reference, the interactions both inside the group of mathematicians, e.g., between Delft and Paris, Delft and the MIT, Delft and Prague, Delft and Leningrad, Princeton and Zürich, and the group of physicists (Einstein-Pauli-Eddington, Kleinde Broglie, Einstein-Reichenbächer, Einstein-Mandel) was more intensive than the interaction between mathematicians and physicists (Weyl-Einstein, Einstein-Cartan, Eddington-Schouten, Kaluza-Einstein, Weyl-Pauli, Schouten-Pauli). Mathematicians often used unified field theory as a motivation for their research. Within the communications-net of mathematicians and theoretical physicists contributing to unified field theory, Schouten played a prominent role. It was unknown to me that he and Friedman in Petersburg, the discoverer of exact solutions of Einstein's equations describing an expanding universe, wrote a joint paper on a unified field theory with vector torsion, as we would say today. Schouten published also in a physics journal, the Zeitschrift für Physik. From the mutual references to their papers, among mathematicians Weyl, Cartan, Schouten, Eisenhart, Veblen, T. Y. Thomas, J. M. Thomas, Levi-Civita, Berwald, Weitzenböck,

\footnotetext{
316 Ludwig Berwald (1883-1942). Born in Prague. Studied mathematics in Munich and became a full professor at the German Charles University in Prague. His scientific work is mainly in differential geometry, notably on Finsler geometry and on spray geometry, i.e., path spaces. He died in Poland after having been deported by the German authorities just because he was Jewish.

317 "Die Theorie der Differentialformen (Tensoren) hat mit Rücksicht auf ihre Verwendung in modernen physikalischen Theorien in den letzten Jahren eine ausführliche Bearbeitung erfahren. Wir erwähnen Ricci, Levi-Civita, Hessenberg, Einstein, Hilbert, [Felix] Klein, E. Noether, Weyl."
}

Living Reviews in Relativity

http: //www . livingreviews . org/lrr-2004-2 
and later Hlavatý and Vranceanu stand out. In the following, we give a few examples of interaction among mathematicians. From Schouten's acknowledgment,

"I owe thanks to Mr. L. Berwald in Prague, with whom I had an intensive exchange of ideas from September 1921, and who was so friendly as to give me his manuscripts before they went into print." 318 [299]

we note that he had intensive contact with Berwald in Prague. Later, while working on spinors, Schouten interacted with Veblen in Princeton, such that the latter referred to him:

"[This note] suggested itself as a possible basis for a geometrical interpretation of Eddington's theory of the interaction of electric charges [61] and was proposed to Prof. Schouten during his visit to America as a possible geometrical interpretation of the theory of spin-quantities which he was then developing." ([306]; Schouten had Hlavaty as a co-author [312])

Among Schouten's correspondents were theoretical physicists as well, such as Pauli. In a paper on "space time and spin space" Schouten acknowledged that

"Mr. Pauli was so friendly as to permit me to quote this theorem from an unpublished manuscript." 319 ([308], p. 406, footnote 4)

and

"A correspondence with Mr. Pauli induced me to investigate this invariance." ${ }^{320}$ ([308], p. 414, footnote 1)

We also noticed in Section 5 that Schouten wrote his papers on the classification of linear connections [297, 296] with the explicit intention of attracting readers from physics.

On the one hand, Einstein must have been best informed by receiving papers, books, and the latest news, or even visits from many of his active colleagues. On the other, he rarely referred to these papers and books; as far as I am aware his extended correspondence included Eisenhart, Eddington, Kaluza, Mandel, Pauli, Veblen, A. Wenzl, Weyl, and Zaycoff, but not Schouten, J. M. Thomas, T. Y. Thomas, O. Klein, not to speak of Reichenbächer. In terms of his scientific output in the area of unified field theory, a more precise description of the balance between Einstein's being at the receiving end and his stimulating and creative role will have to be given in the future.

\footnotetext{
318 "Herrn L. Berwald in Prag, mit dem ich seit September 1921 in regem Gedankenaustausch stand und der mir seine Manuskripte schon vor der Drucklegung freundlichst zur Verfügung stellte, schulde ich Dank."

319 "Herr Pauli hatte die Freundlichkeit, zu erlauben, diesen Satz aus einem noch nicht publizierten Manuskript zu zitieren."

320 "Eine Korrespondenz mit Herrn Pauli veranlasste mich, diese Invarianz zu untersuchen."
} 


\section{Public Reception of Unified Field Theory at the Time}

We must distinguish between the acceptance of unified field theory in academic circles and its reverberations in the general press. Of course, Weyl could further his own theory through his well received book [396]. His unification of electricity and gravitation was also given room in Eddington's monograph "Space, Time, and Gravitation" of 1920 [57]. In German scientific handbooks on physics and monographs on relativity theory at the time, there are long articles on special and general relativity, but unified field theory is mentioned only in passing, and with the names of Weyl, Eddington, and Kaluza stressed instead of Einstein's ([194], pp. 576-579, and [9], pp. 378-383). Perhaps, it was too early for Born to mention Weyl in his book on Einstein's relativity theory of 1920 [19]. In the third edition of 1922, he entered a single sentence after he had lauded Einstein for his derivation of the field equations of general relativity:

"Hilbert, Klein [i.e., Felix Klein] and other mathematicians have taken part, have researched in depth and illuminated the formal structure of Einstein's formulas." 321 ([20], p. 248)

After Pauli's handbook article, Weyl hardly could have been overlooked. Nevertheless, the 2nd volume of Laue's book did not even mention Weyl's theory, nor did Levi-Civita's book in its English or German versions of 1927 and 1928 [387, 205]. In contrast, Schouten's and Eddington's monographs treated Weyl extensively [300, 60]. Already in 1921, Fabre's weak presentation of "Les théories d'Einstein" [125] had an admiring but irrelevant appendix on Weyl's unified field theory, while, in 1922, J. Becquerel in Paris and E. Neumann in Marburg in their books on relativity had brief presentations of Weyl's theory [236, 10]. One or the other philosopher of science took into account some of the developments, mostly Weyl's theory, as did Reichenbach in 1928 [267]. In his book on "The present world view according to the natural sciences", Wenzl discussed Einstein's theory of distant parallelism but cautioned that this theory, as a physical theory was not on the same level as general relativity: It seemed unclear whether the new theory would predict new phenomena that could be empirically tested [394].

As to the impact on the public at large, Einstein's fame as the creator of the empirically tested general relativity continued to shine over his successive attempts at unified field theory. Chandarsekhar reports an after-dinner chat in 1933 in which Rutherford made Eddington responsible for Einstein's fame; he nailed it to the headlining, in the British papers, of the meeting of the Royal Society, at which the results of the British Solar Eclipse Expedition were reported:

"[...] the typhoon of publicity crossed the Atlantic. From that point on, the American press played Einstein to the maximum." ([42], p. 28)

Also, from 1930, Einstein's yearly travels to the United States and his sojourns there brought about increased publicity for his research on unified field theory. Thus, a "preliminary announcement for the Josiah Macy, Jr., Foundation" of his paper with his assistant Mayer on the 5-vector formulation of Kaluza's theory [107] was printed in full in Science about a month before the publication appeared [94]. However, it seems safe to say that reports on Einstein's newest unified field theory in the dailies, whether seen as an educational affair or as part of entertainment, must have strained the general public's intellectual abilities.

\footnotetext{
321 "Hilbert, Klein [i.e., Felix Klein], Weyl und andere Mathematiker haben dabei mitgewirkt und die formale
} Struktur der Einsteinschen Formeln tief durchforscht und aufgehellt."

Living Reviews in Relativity

http: //www. livingreviews.org/lrr-2004-2 


\section{Conclusion}

Even a superficial survey such as the one made here shows clearly the dense net of mathematicians and theoretical physicists involved in the building of unified field theory and of the geometric structures underlying it. Mathematician Grossmann introduced physicist Einstein into Ricci's calculus; Einstein influenced many mathematicians such as Hessenberg, Weyl, Schouten, Struik, Cartan, Eisenhart, and Veblen, to name a few. In return, some very influential ideas on Einstein's path within unified field theories came from these mathematicians: Förster's asymmetric metric ${ }^{322}$, Cartan's distant parallelism, Kaluza's five-dimensional space, Weyl's, Schouten's, and Cartan's completely general concept of connection, Veblen's projective formulation.

My greatest surprise was to learn that, in the period considered here, in the area of unified field theories, Einstein did not assume the role of conceptual leader that he had played when creating general relativity. In fact, in the area of unified field theories, he tended to re-invent mathematical developments made before. The ideas most fruitful for physics in the long run came from Weyl ("gauge concept"), Kaluza ("extension of number of space dimensions"), and O. Klein ("compactification") "323. Vizgin states that Einstein, around 1923 to 1930

"became the recognised leader of the investigations [in unified field theory], taking over, as it were, the baton from Weyl, who had been the leading authority for the previous five years." ([385], p. 183)

This may be true, but in a sense possibly not intended by Vizgin. First, Einstein could lead only those few unaffected by the main new topic of theoretical physics at the time: quantum theory. Second, Einstein's importance consisted in having been the central identification figure in a scientific enterprise within theoretical physics which, without his weight, fame, and obstinacy, would have been dwindling to an interesting specialty in differential geometry and become a dead end for physicists. It is interesting, though, to note how uncritically Einstein's zig-zagging path through the wealth of constructive possibilities was followed by many in the (small) body of researchers in the field.

Cartan saw it positively:

"One can see [...] the variety of aspects by which unified field theory may be envisaged, and also the difficulty of the problems arising from it. But Mr. Einstein is not one of those afraid of difficulties; even if his attempt does not succeed, it will have forced us to think about the great questions at the foundation of science." 324 ([36], p. 1184/1185)

If there is an enigma left in the scientific part of Einstein's life, then it occurs here, in the area of unified field theory. As judged from his ambitious goals and as seen from the aspect of lasting scientific value, not only did Einstein's endeavours - from the affine approach to the teleparallel theory - lead nowhere, but sometimes they were also quite behind what others knew already, as in Kaluza-Klein theory and in the area of spinors. Einstein also was not very fruitful in developing, conceptually, his particle model beyond the image of regular field concentrations. Einstein knew only too well how disconnected his various unified field theories were from the possibility of them being checked empirically. He, and everybody else working in the field, did not succeed in extracting from one of the unified field theories an example of a new physical effect of gravito-electromagnetic nature to be tested by experiment or observation. Unlike in his previous scientific career where he was most ingenious and prolific in devising (thought-) experiments, now Einstein's physical

\footnotetext{
${ }^{322}$ Förster wrote his thesis in mathematics [137].

${ }^{323}$ For the historical development of gauge theory from the point of view of physics, cf. Straumann [335, 240].

324 "On voit [...] la variété des aspects sous lesquels peut etre envisagée la théorie unitaire du champ et aussi la difficulté des problèmes qu'elle soulève. Mais M. Einstein n'est pas de ceux à qui les difficultés font peur et, meme si sa tentative n'aboutit pas, elle nous aura forcés à réfléchir sur les grandes questions qui sont à la base de la science."
} 
intuition seems to have been buried under formal structural thinking. Let us quote a remark he made with regard to his theory of distant parallelism, but which could equally well stand for all the other unified field theories he tried:

"At present, this new theory is nothing but a mathematical construct barely connected to physical reality by very loose cords. It has been discovered by exclusively formal considerations, and its mathematical consequences have not yet been developed sufficiently for allowing a comparison with experiment. Nevertheless, to me this attempt seems very interesting in itself; it mainly offers splendid possibilities for the [further] development, and it is with the hope that the mathematicians get interested in it that I permit myself to expose and analyse [the theory] here." ${ }^{325}$ ([92], p. 1)

Then, why did he so obstinately follow this line of research and isolate himself from most of his peers, except Schrödinger and, perhaps, de Broglie? Was it an unfailing belief that "geometrizable fields" must play the fundamental role as compared to particles ${ }^{326}$ ? Just what, besides his appreciation of the power of mathematics, supported his hope that, with one sweep, quantum theory and classical field theory could be brought into a single representation? It seems that, in the second half of his life, Einstein more and more came to think that the structure of physical theories may be unraveled by the hypothetical-deductive approach alone, without assistance from any empirical input:

"[...], in the end experience is the only competent judge. Yet in the meantime one thing may be said in defence of the theory. Advances in scientific knowledge must bring about the result that an increase in formal simplicity can only be won at the cost of an increased distance or gap between the fundamental hypotheses of the theory on the one hand, and the directly observed facts on the other. Theory is compelled more and more from the inductive to the deductive method, even though the most important demand to be made of every scientific theory will always remain that it must fit the facts." ([86], pp. 114-115)

It may also be that general relativity, his great and lasting success in dealing with gravitation, was misleading him. In a report about his teleparallel geometry, after having described "the derivation of the complicated field law of gravitation along a logical path," Einstein went on to say:

"The successful attempt to derive delicate laws of nature, along a purely mental path, by following a belief in the formal unity of the structure of reality, encourages continuation in this speculative direction, the dangers of which everyone vividly must keep in sight who dares follow it." 327 [87]

After 1926, Einstein more and more removed himself from a working knowledge of quantum mechanics, not to speak of quantum field theory. Although he sensed his growing isolation from the physics mainstream, he downplayed it by a good measure of self-confidence. In connection with the field equations of his new geometry with distant parallelism, in a letter to his friend Besso, Einstein rated his efforts this way:

\footnotetext{
325 “À l'heure actuelle cette nouvelle théorie n'est qu'un édifice mathématique, à peine relié par quelques liens très lâches à la réalité physique. Elle a été découverte par des considérations exclusivement formelles et ses conséquences mathématiques n'ont pas pu être suffisamment développées pour permettre la comparaison avec l'expérience. Néanmoins, cette tentative me semble très intéressante en elle-même; elle offre surtout de magnifique possibilités de développemnet et c'est dans l'espoir que les mathématiciens s'y intéressont, que je me permets de l'exposer et de l'analyser ici."

${ }^{326}$ Compare with the fate of contemporaneaous (quantum-) geometrodynamics [328].

327 "Das Gelingen dieses Versuches, aus der Überzeugung der formalen Einheit der Struktur des Wirklichen heraus auf rein gedanklichem Wege subtile Naturgesetze abzuleiten, ermutigt zu einem Fortschreiten auf diesem spekulativen Wege, dessen Gefahren sich jeder lebhaft vor Augen halten muss, der ihn zu beschreiten wagt."
} 
"This looks old-fashioned, and the dear colleagues and also you, my dear, will show me the tongue as long as they can. Because Planck's $h$ is not showing up in the equations. But if the limit is clearly reached of what the statistical fad can achieve they will again return full of repentance to the space-time picture, and then these equations will form a starting point." 328 ([99], p. 240)

Even seventy years later, with a limit to the "statistical fad" not yet reached, Einstein's world fame is strong enough as to induce people to continue his path toward classical unified field theory. They do this with only slightly changed methodology, but with a greatly enlarged technical toolbox, and despite a lasting lack of empirical means for deciding whether such attempts of bringing progress for the understanding of nature are valid, or not. One powerful and intriguing new instrument in the toolbox, which was not available to Einstein, is supersymmetry. By it fermion fields can also be "geometrized" 329 . The argument put forward in favor of such a continuation is now much the same as that advanced by K. Novobatzky in 1931:

"In the present situation, neither from classical nor from quantum mechanical methods alone 'all' can be expected; rather, it suits us to adopt the opinion, voiced several times, that the field problem must be carried further on classical ground before it may present new anchor points for quantum mechanics. Seen precisely from this angle, it is regrettable that after these broad designs, such as the ones available in gauge theory and distant parallelism, no further attempts in the classical direction can be noticed." 330 ([239], p. 683)

It might be an interesting task to confront the methodology that helped Einstein to arrive at general relativity with the one used by him within unified field theory. (See the contributions of J. Renn, J. Norton, M. Janssen, T. Sauer, M. Schemmel, et al. originating from their work on Einstein's Zürich notebook of 1912 [279, 280].) If it is the same, then it might become harder to draw general conclusions as to its importance for the gain in and development of knowledge in physics.

A report on the rich further development of the field past 1933 will be given in Part II of this review.

\footnotetext{
328 "Das sieht altertümlich aus und die lieben Kollegen sowie auch Du, mein Lieber, werden zunächst einmal die Zunge herausstrecken solange es geht. Denn in diesen Gleichungen kommt kein Planck'sches $h$ vor. Aber wenn man an die Leistungsgrenzen des statistischen Fimmels deutlich gelangt sein wird, wird man wieder zur zeiträumlichen Auffassung reuevoll zurückkehren und dann werden diese Gleichungen einen Ausgangspunkt bilden."

${ }^{329} \mathrm{My}$ reservations hold only if the toolbox does not also contain quantum field theory.

330 "Nach dem heutigen Stand der Dinge wird man weder von klassischen noch von quantentheoretischen Methoden 'alles' erwarten, sondern sich eher jener mehrfach geäusserten Meinung anschliessen, dass das Feldproblem auf klassischem Boden weitergeführt werden muss, ehe es der Quantentheorie neuere Angriffspunkte zu bieten vermag. Eben deshalb ist es bedauerlich, dass nach jenen gross angelegten Entwürfen, wie sie in der Eichungstheorie und im Fernparallelismus vorliegen, weitere Versuche in klassischer Richtung nicht zu vermerken sind."
} 


\section{Acknowledgements}

The carefully worded and detailed comments of a referee have been helpful in improving on the original version. I would also like to thank the staff of Living Reviews, notably Mrs. Christina Weyher and Mr. Robert Forkel, for their friendly and generous assistance. 


\section{References}

[1] "MacTutor History of Mathematics Archive", web interface to database, University of St Andrews. URL (cited on 28 January 2003):

http://turnbull.mcs.st-and.ac.uk/history/. 1.1

[2] "News and Views", Nature, 123(3092), 174-175, (1929). [DOI]. 6.4.2

[3] Anderson, W., "Gewöhnliche Materie und strahlende Energie als verschiedene 'Phasen' eines und desselben Grundstoffes", Z. Phys., 54, 433-444, (1929). [DOI]. 7.1

[4] Bach, R., "Zur Weylschen Relativitätstheorie und der Weylschen Erweiterung des Krümmungsbegriffs", Math. Z., 9, 110-135, (1921). [DOI]. 58, 4.1.1, 9

[5] Bargmann, V., "Über eine Verallgemeinerung des Einsteinschen Raumtyps", Z. Phys., 65, 830-847, (1930). [DOI]. 225, 6.4.5

[6] Bargmann, V., "Bemerkungen zur allgemein-relativistischen Fassung der Quantentheorie", Sitzungsber. Preuss. Akad. Wiss., 1932(XXIV), 346-354, (1932). 7.2.1, 7.2.2, 7.2.4

[7] Bargmann, V., "Über den Zusammenhang zwischen Semivektoren und Spinoren und die Reduktion der Diracgleichungen für Semivektoren", Helv. Phys. Acta, 7, 57-82, (1934). 7.3

[8] Bargmann, V., "Relativity", in Fierz, M., and Weisskopf, V.F., eds., Theoretical Physics in the Twentieth Century: A Memorial Volume to Wolfgang Pauli, pp. 187-198, (Interscience Publishers, New York, 1960). 140

[9] Beck, G., "Allgemeine Relativitätstheorie", in Geiger, H., and Scheel, K., eds., Handbuch der Physik, Vol. 4, pp. 299-407, (Springer, Berlin, 1929). 10

[10] Becquerel, J., Le Principe de relativité et la théorie de la gravitation, (Gauthier-Villars, Paris, 1922). 10

[11] Bergia, S., "Attempts at Unified Field Theories (1919-1955). Alleged Failure and Intrinsic Validation/Refutation Criteria", in Earman, J., Janssen, M., and Norton, J.D., eds., The Attraction of Gravitation: New Studies in the History of General Relativity, Einstein Studies, vol. 5, pp. 274-307, (Birkhäuser, Boston; Basel, 1993). [Google Books]. 1.1

[12] Bergmann, P.G., "Unitary Field Theory: Yesterday, Today, Tomorrow", in Treder, H.-J., ed., Einstein-Centenarium 1979, Ansprachen und Vorträge auf der Festveranstaltung des Einstein-Komitees der DDR bei der Akademie der Wissenschaften der DDR vom 28.2.-2.3. 1979 in Berlin, (Akademie-Verlag, Berlin, 1979). 1.1

[13] Berwald, L., "Differentialinvarianten in der Geometrie. Riemannsche Mannigfaltigkeiten und ihre Verallgemeinerungen", in Meyer, W.F., and Mohrmann, H., eds., Enzyklopädie der Mathematischen Wissenschaften mit Einschluss ihrer Anwendungen, Band III, 3. Teil, D 11, pp. 73-181, (Teubner, Leipzig, 1923). Related online version (cited on 30 October 2009): http://gdz.sub. uni-goettingen.de/dms/load/img/?PPN=PPN360610161\&DMDID= dmdlog384. 9

[14] Biezunski, M., La diffusion de la théorie de la relativité en France, Ph.D. Thesis, (Université VII, Département de Didactique des Sciences physiques et de la Technologie, Paris, 1981). 6.4 .3 
[15] Biezunski, M., "Inside the coconut: The Einstein-Cartan discussion", in Howard, D., and Stachel, J., eds., Einstein and the History of General Relativity, Based on the proceedings of the 1986 Osgood Hill Conference, North Andover, Massachusetts, 8 - 11 May 1986, Einstein Studies, vol. 1, pp. 315-324, (Birkhäuser, Boston; Basel, 1989). 223

[16] Biezunski, M., Histoire de la physique moderne, (La Découverte, Paris, 1993). 1.2

[17] Birkhoff, G.D., "The hydrogen atom and the Balmer formula", Proc. Natl. Acad. Sci. USA, 13, 165-169, (1927). [DOI]. 8

[18] Birkhoff, G.D., "A theory of matter and electricity", Proc. Natl. Acad. Sci. USA, 13, 160165, (1927). [DOI]. 8

[19] Born, M., Die Relativitätstheorie Einsteins und ihre physikalischen Grundlagen, (Springer, Berlin, 1920). 3.2, 10

[20] Born, M., Die Relativitätstheorie Einsteins und ihre physikalischen Grundlagen, (Springer, Berlin, 1922), 2nd edition. 10

[21] Born, M., "Quelques problèmes de méchanique quantique", Ann. Inst. Henri Poincare, 1, 205-284, (1930). 6.4.3

[22] Bortolotti, E., "On metric connections with absolute parallelism", Proc. K. Akad. Wetensch., 30, 216-218, (1927). 6.4.5

[23] Bortolotti, E., "Parallelismo assoluto nella varietá a connessione affine e nuove veduti sulla relativitá", Mem. Accad. Sci. Ist. Bologna, Cl. Sci. Fis., 6, 45-58, (1928). 6.4.5

[24] Bortolotti, E., "Stelle di congruenze e parallelisme assoluto: basi geometriche di una recente teoria di Einstein", Rend. Lincei, 9, 530-538, (1929). 6.3.2, 6.4.5

[25] Bortolotti, E., "Stelle di congruenze e parallelisme assoluto: basi geometriche di una recente teoria di Einstein", Rend. Lincei, 9, 530-538, (1929). 6.4.5

[26] Boyd, P.T., Centrella, J.M., and Klasky, S.A., "Properties of gravitational 'solitons"', Phys. Rev. D, 43, 379-90, (1991). 1.2

[27] Buchdahl, H.A., "Gauge-invariant generalization of field theories with asymmetric fundamental tensor", Quart. J. Math., 8, 89-96, (1957). [DOI]. 80

[28] Cao, T.Y., Conceptual developments of 20th century field theories, (Cambridge University Press, Cambridge, 1997). 1.2

[29] Cartan, É., "Sur les équations de structure des espaces généralisés et l'Univers optique", $C$. R. Acad. Sci., 174, 857-859, (1922). 3

[30] Cartan, É., "Sur les espaces généralisés et la théorie de la relativité", C. R. Acad. Sci., 174, 734-737, (1922). 6.4.1

[31] Cartan, É., "Sur les variétés à connexion affine courbure de Riemann et les espaces à torsion", C. R. Acad. Sci., 174, 593-595, (1922). 6.4.1

[32] Cartan, É., "Sur les variétés à connexion affine et la théorie de la relativité généralisée", Ann. Sci. Ecole Norm. Sup., 40, 325-412, (1923). 5, 6.4.1

[33] Cartan, É., "Sur les espaces de Riemann dans lesquels le transport par parallélism conserve la courbure", Rend. Lincei, 3(6), 544-547, (1926). 6.4.1 
[34] Cartan, É., "La Géométrie des groupes de transformations", J. Math. Pures Appl., 6, 1-119, (1927). 6.4.1

[35] Cartan, É., "Notice historique sur la notion de parallélisme absolu", Math. Ann., 102, 698706, (1930). [DOI]. 6.4.1, 6.4.3

[36] Cartan, É., "Le parallelisme absolu et la théorie unitaire du champ", Rev. Metaphys. Morale, 38, 13-28, (1931). Oeuvres Complètes, tome III, 2 pp. 1167-1185. 3.2, 11

[37] Cartan, É., "Sur la théorie des systèmes en involution et ses applications à la Relativité", Bull. Soc. Math. France, 59, 88-118, (1931). 6.3.2, 252

[38] Cartan, É., Oeuvres Complètes, (Gauthier-Villars, Paris, 1950). 3 parts with 2 vols. each, 1950-1955. 24

[39] Cartan, É., "La théorie unitaire d'Einstein-Mayer", in Oeuvres Complètes, Vol. III/2, pp. 1863-1875, (Gauthier-Villars, Paris, 1955). manuscrit datant de 1934 environ. 6.3.2

[40] Cartan, É., and Schouten, J.A., "On Riemaniann geometries admitting an absolute parallelism", Proc. K. Akad. Wetensch., 29, 933-946, (1926). 6.4.1

[41] Cartan, É., and Schouten, J.A., "On the Geometry of the Group-manifold of Simple and Semi-simple Groups", Proc. K. Akad. Wetensch., 29, 803-815, (1926). 6.4.1

[42] Chandrasekhar, S., Eddington: The most distinguished astrophysicist of his time, (Cambridge University Press, Cambridge, 1983). [Google Books]. 10

[43] Darrieus, M.G., "Sur une form remarquable des équations de Maxwell-Lorentz dans l'univers à cinq dimensions", J. Phys. Radium, 8, 444-446, (1927). [DOI]. 6.3.1

[44] Darwin, C.G., "The wave equations of the electron", Proc. R. Soc. London, Ser. A, 118, 654-680, (1928). 7.1

[45] Darwin, C.G., "La théorie ondulatoire de la matière", Ann. Inst. Henri Poincare, 1, 25-51, (1930). 6.4.3

[46] de Broglie, L., "L'univers à cinq dimensions et la mécanique ondulatoire", J. Phys. Radium, 8, 65-73, (1927). [DOI]. 6.3.1

[47] de Broglie, L., "Réponse à la note de M. O. Klein", J. Phys. Radium, 8, 244, (1927). [DOI]. 6.3 .1

[48] de Donder, T.E., "Généralisation relativiste de la nouvelle théorie d'Einstein", C. R. Acad. Sci., 187, 817-819, (1928). 6.4.3, 6.4.5

[49] De Sabbata, V., and Schmutzer, E., eds., Unified field theories in more than 4 dimensions, Proceedings of the International School of Cosmology and Gravitation, held at Erice, Italy, 20 May-1 June 1982, (World Scientific, Singapore, 1983). 102, 4.2

[50] Debever, R., ed., Élie Cartan - Albert Einstein: Lettres sur le parallélisme absolu 1929-1932, (Academie Royale de Belgique, Brussels, 1979). 6.4.1, 6.4.3, 6.4.3, 6.4.3, 6.4.4, 6.4.6

[51] Dienes, P., "Sur la théorie électromagnétique relativiste", C. R. Acad. Sci., 176, 238-241, (1923). 4.1.5

[52] Dirac, P.A.M., "The quantum theory of emission and absorption of radiation", Proc. R. Soc. London, Ser. A, 114, 243-265, (1927). 1.2 
[53] Dirac, P.A.M., "The quantum theory of the electron", Proc. R. Soc. London, Ser. A, 117, 610-624, (1928). 2.1.5.2

[54] Dirac, P.A.M., "The quantum theory of the electron", Proc. R. Soc. London, Ser. A, 118, 351-361, (1928). 2.1.5.2

[55] Dirac, P.A.M., "A Theory of Electrons and Protons", Proc. R. Soc. London, Ser. A, 126, 360-365, (1930). 12, 309

[56] Dirac, P.A.M., "Quantised Singularities in the Electromagnetic Field", Proc. R. Soc. London, Ser. A, 133, 60-72, (1931). 12

[57] Eddington, A.S., Space, Time, and Gravitation: An outline of the general relativity theory, (Cambiridge University Press, Cambridge, 1920). 4.1.4, 10

[58] Eddington, A.S., "A generalisation of Weyl's theory of the electromagnetic and gravitational fields", Proc. R. Soc. London, Ser. A, 99, 104-122, (1921). 4.3.1, 4.3.1, 4.3.1, 5

[59] Eddington, A.S., The mathematical theory of relativity, (Cambridge University Press, Cambridge, 1924), 2nd edition. 1st edition 1923. 2.1.1, 4.1.4, 4.1.5

[60] Eddington, A.S., Relativitätstheorie in mathematischer Behandlung, (Springer, Berlin, 1925). $4.3 .3,5,6.2,10$

[61] Eddington, A.S., "A symmetrical treatment of the wave equation", Proc. R. Soc. London, Ser. A, 121, 524-542, (1928). 7.2.4, 7.2.4, 9

[62] Eddington, A.S., "Einstein's Field-Theory", Nature, 123(3095), 280-281, (1929). [DOI]. 6.4.4, 6.4 .5

[63] Eddington, A.S., The Expanding Universe, (Cambridge University Press, Cambridge, 1933). 4.3.1

[64] Eddington, A.S., The Mathematical Theory of Relativity, (Cambridge University Press, Cambridge, 1960). Reprint of the 1st ed. 1923. 4.3.1, 4.3.3

[65] Ehlers, J., "The nature and structure of space-time", in Mehra, J., ed., The Physicists Conception of Nature, Symposium held at ICTP, Miramare, Trieste, Italy, 18 - 25 September 1972, (Reidel, Dordrecht; Boston, 1973). 2.1.3.2

[66] Ehlers, J., "Über den Newtonschen Grenzwert der Einsteinschen Gravitationstheorie", in Nitsch, J., Pfarr, J., and Stachow, E.-W., eds., Grundlagenprobleme der modernen Physik: Festschrift für Peter Mittelstaedt zum 50. Geburtstag, (Bibliographisches Institut, Mannheim, 1981). 2.1.3.2

[67] Ehlers, J., Pirani, F.A.E., and Schild, A., "The Geometry of Free Fall and Light Propagation", in O'Raiffeartaigh, L., ed., Studies in Relativity: Papers in honour of J. L. Synge, pp. 63-84, (Clarendon Press, Oxford, 1972). 2.1.1, 2.1.3.2

[68] Ehrenfest, P., "Einige die Quantenmechanik betreffende Erkundigungsfragen", Z. Phys., 78, 555-559, (1932). [DOI]. 7.2.1

[69] Einstein, A., "Letter to De Haas, May 7, 1933", personal communication. 7.3 
[70] Einstein, A., "Spielen Gravitationsfelder im Aufbau der materiellen Elementarteilchen eine wesentliche Rolle?", Sitzungsber. Preuss. Akad. Wiss., 1919(XX), 349-356, (1919). Related online version (cited on 30 October 2009):

http://einstein-annalen.mpiwg-berlin.mpg.de/related_texts/sitzungsberichte/ 69YV118A. 3.2, 69, 4, 6.1

[71] Einstein, A., "Antwort auf vorstehende Betrachtung", Die Naturwissenschaften, 8, 1010 1011, (1920). [DOI]. 3.1

[72] Einstein, A., "Geometrie und Erfahrung", Sitzungsber. Preuss. Akad. Wiss., 1921(V), 123130, (1921). 3.2, 4

[73] Einstein, A., "Über eine naheliegende Ergänzung des Fundamentes der allgemeinen Relativitätstheorie", Sitzungsber. Preuss. Akad. Wiss., 1921(XII), 261-264, (1921). Related online version (cited on 30 October 2009):

http://einstein-annalen.mpiwg-berlin.mpg.de/related_texts/sitzungsberichte/ QAB7NNDB. 4.1.5

[74] Einstein, A., "Bemerkungen zu meiner Arbeit 'Zur allgemeinen Relativitätstheorie", Sitzungsber. Preuss. Akad. Wiss., 1923(XII), 76-77, (1923). Related online version (cited on 30 October 2009):

http://einstein-annalen.mpiwg-berlin.mpg.de/related_texts/sitzungsberichte/ EVSSPDP8. 4.3.2, 5,9

[75] Einstein, A., "Bietet die Feldtheorie Möglichkeiten zur Lösung des Quantenproblems?", Sitzungsber. Preuss. Akad. Wiss., 1923, 359-364, (1923). Related online version (cited on 30 October 2009):

http://einstein-annalen.mpiwg-berlin.mpg.de/related_texts/sitzungsberichte/ MXYDBZW5. 4.3.4

[76] Einstein, A., "Zur affinen Feldtheorie", Sitzungsber. Preuss. Akad. Wiss., 1923(XVII), 137-140, (1923). Related online version (cited on 30 October 2009):

http://einstein-annalen.mpiwg-berlin.mpg.de/related_texts/sitzungsberichte/ GBEHMB2D. 4.3.2, 9

[77] Einstein, A., "Zur allgemeinen Relativitätstheorie", Sitzungsber. Preuss. Akad. Wiss., 1923(V), 32-38, (1923). Related online version (cited on 30 October 2009):

http://einstein-annalen.mpiwg-berlin.mpg.de/related_texts/sitzungsberichte/ B98WXTVX. 42, 4.3.2, 4.3.2, 5,9

[78] Einstein, A., "Einheitliche Feldtheorie von Gravitation und Elektrizität", Sitzungsber. Preuss. Akad. Wiss., 1925(XXII), 414-419, (1925). Related online version (cited on 30 October 2009):

http://einstein-annalen.mpiwg-berlin.mpg.de/related_texts/sitzungsberichte/ ZHDAXTXQ. 6.1

[79] Einstein, A., "Elektron und allgemeine Relativitätstheorie", Physica, 5, 330-334, (1925). 6.1

[80] Einstein, A., "Über die formale Beziehung des Riemannschen Krümmungstensors zu den Feldgleichungen der Gravitation", Math. Ann., 97, 99-103, (1927). [DOI]. 6.1

[81] Einstein, A., "Zu Kaluzas Theorie des Zusammenhangs von Gravitation und Elektrizität. Erste Mitteilung", Sitzungsber. Preuss. Akad. Wiss., 1927(VI), 23-25, (1927). Related online version (cited on 30 October 2009): 
http://einstein-annalen.mpiwg-berlin.mpg.de/related_texts/sitzungsberichte/ 75ASGAHY. 6.3 .1

[82] Einstein, A., "Zu Kaluzas Theorie des Zusammenhangs von Gravitation und Elektrizität. Zweite Mitteilung", Sitzungsber. Preuss. Akad. Wiss., 1927(VI), 26-30, (1927). Related online version (cited on 30 October 2009):

http://einstein-annalen.mpiwg-berlin.mpg.de/related_texts/sitzungsberichte/ 75ASGAHY. 6.3.1

[83] Einstein, A., "Neue Möglichkeit für eine einheitliche Feldtheorie von Gravitation und Elektrizität", Sitzungsber. Preuss. Akad. Wiss., 1928(XVIII), 224-227, (1928). 6.4.1, 6.4.3, 6.4.4, $6.4 .5,6.4 .5,6.4 .5$

[84] Einstein, A., "Riemann-Geometrie mit Aufrechterhaltung des Begriffes des Fernparallelismus", Sitzungsber. Preuss. Akad. Wiss., 1928(XVII), 217-221, (1928). Related online version (cited on 30 October 2009):

http://einstein-annalen.mpiwg-berlin.mpg.de/related_texts/sitzungsberichte/ B8AG2G5E. 6.4.1, 6.4.3, 6.4.4, 6.4.5, 6.4.5, 6.4.5, 7.2.3

[85] Einstein, A., "Einheitliche Feldtheorie und Hamiltonsches Prinzip", Sitzungsber. Preuss. Akad. Wiss., 1929(X), 156-159, (1929). Related online version (cited on 30 October 2009): http://einstein-annalen.mpiwg-berlin.mpg.de/related_texts/sitzungsberichte/ EVNERTBY. 6.4.3, 6.4.5

[86] Einstein, A., "The New Field Theory I, II", Observatory, 51, 82-87, (1929). 6.4.2, 11

[87] Einstein, A., "Über den gegenwärtigen Stand der Feldtheorie", in Honegger, E., ed., Festschrift, Prof. Dr. A. Stodola zum 70. Geburtstag, Überreicht von seinen Freunden und Schülern, (Orell Füssli, Zurich, 1929). 6.4.3, 11

[88] Einstein, A., "Zur einheitlichen Feldtheorie", Sitzungsber. Preuss. Akad. Wiss., 1929(I), 2-7, (1929). 6.4.2, 6.4.3, 199, 201, 6.4.3, 6.4.3, 6.4.4, 6.4.5, 6.4.5, 7.2.3

[89] Einstein, A., "Auf die Riemann-Metrik und den Fernparallelismus gegründete einheitliche Feldtheorie", Math. Ann., 102, 685-697, (1930). [DOI]. 6.4.1, 6.4.3, 6.4.3, 6.4.4, 231, 6.4.5

[90] Einstein, A., "Die Kompatibilität der Feldgleichungen in der einheitlichen Feldtheorie", Sitzungsber. Preuss. Akad. Wiss., 1930(I), 18-23, (1930). Related online version (cited on 30 October 2009):

http://einstein-annalen.mpiwg-berlin.mpg.de/related_texts/sitzungsberichte/ NMS7SWKE. 6.4.3, 6.4.3, 6.4.6

[91] Einstein, A., "Professor Einstein's adress at the University of Nottingham", Science, 71, 608-611, (1930). 1.2, 6.4.3

[92] Einstein, A., "Théorie unitaire du champs physique", Ann. Inst. Henri Poincare, 1, 1-24, (1930). 6.4.3, 11

[93] Einstein, A., "Zur Theorie der Räume mit Riemann-Metrik und Fernparallelismus", Sitzungsber. Preuss. Akad. Wiss., 1930(VI), 401-402, (1930). Related online version (cited on 30 October 2009):

http://einstein-annalen.mpiwg-berlin.mpg.de/related_texts/sitzungsberichte/ K17BZ9SW. 6.4.3, 6.4.3, 6.4.5 
[94] Einstein, A., "Gravitational and electromagnetic fields", Science, 74, 438-439, (1931). 6.3.2, 10

[95] Einstein, A., "Maxwell's influence on the development of the conception of physical reality", in James Clerk Maxwell: A Commemoration Volume 1831-1931, (Cambridge University Press, Cambridge, 1931). 1.2

[96] Einstein, A., "Der gegenwärtige Stand der Relativitätstheorie", Die Quelle, 82, 440-442, (1932). 1.2, 6.3.2

[97] Einstein, A., "A Generalization of the Relativistic Theory of Gravitation", Ann. Math., 46, 578-584, (1945). [DOI]. 2.1.3.2, 2.3

[98] Einstein, A., Briefe an Maurice Solovine, (Gauthier-Villars, Paris, 1956). 6.4.3

[99] Einstein, A., Correspondance avec Michele Besso: 1903-1955, (Hermann, Paris, 1972). ed. Speziali, P. 4.1.3, 4.1.4, 4.3.3, 4.3.4, 6.1, 6.1, 6.3.2, 7.3, 11

[100] Einstein, A., "Zur Methodik der Theoretischen Physik", in Albert Einstein: Mein Weltbild, (Ullstein, Frankfurt am Main, 1984). 1st ed. 1934 Amsterdam. 7.3

[101] Einstein, A., "Letter to David Hilbert", in Schulman, R., Kox, A.J., Janssen, M., and Illy, J., eds., Einstein Collected Papers, Vol. 8A, Doc 144, p. 199, (Princeton University Press, Princeton, NJ, 1997). 3.1

[102] Einstein, A., and Bergmann, P., "On a generalization of Kaluza's theory of electricity", Ann. Math., 39, 683-701, (1938). [DOI]. 6.3.1

[103] Einstein, A., Born, H., and Born, M., Albert Einstein, Hedwig und Max Born, Briefwechsel: 1916-1955 / kommentiert von Max Born; Geleitwort von Bertrand Russell; Vorwort von Werner Heisenberg, (Edition Erbrich, Frankfurt am Main, 1982). 4.1.4, 4.3.4, 234, 7.2.3, 313

[104] Einstein, A., and Fokker, A.D., "Nordströmsche Gravitationstheorie vom Standpunkt des absoluten Differentialkalküls", Ann. Phys. (Leipzig), 44, 321-328, (1914). [DOI]. 4.2

[105] Einstein, A., and Grommer, J., "Beweis der Nichtexistenz eines überall regulären zentrisch symmetrischen Feldes nach der Feldtheorie von Kaluza", in Einstein, A., ed., Mathematica et physica, Scripta Hierosolymitana, vol. 1, pp. 1-4, (Hebrew University, Jerusalem, 1923). 4.2

[106] Einstein, A., and Mayer, W., "Zwei strenge statische Lösungen der Feldgleichungen der einheitlichen Feldtheorie", Sitzungsber. Preuss. Akad. Wiss., 1930(VI), 110-120, (1930). Related online version (cited on 30 October 2009): http://einstein-annalen.mpiwg-berlin.mpg.de/related_texts/sitzungsberichte/ GEYDB1K1. 6.4.3, 244

[107] Einstein, A., and Mayer, W., "Einheitliche Theorie von Gravitation und Elektrizität", Sitzungsber. Preuss. Akad. Wiss., 1931(XXV), 541-557, (1931). 6.2, 6.3.2, 6.3.2, 10

[108] Einstein, A., and Mayer, W., "Systematische Untersuchung über kompatible Feldgleichungen, welche in einem Riemannschen Raume mit Fernparallelismus gesetzt werden können", Sitzungsber. Preuss. Akad. Wiss., 1931(XIII), 257-265, (1931). 6.4.3 
[109] Einstein, A., and Mayer, W., "Einheitliche Theorie von Gravitation und Elektrizität. (Zweite Abhandlung.)", Sitzungsber. Preuss. Akad. Wiss., 1932(XII), 130-137, (1932). Related online version (cited on 30 October 2009):

http://einstein-annalen.mpiwg-berlin.mpg.de/related_texts/sitzungsberichte/ TSH25SM0. 6.3 .2

[110] Einstein, A., and Mayer, W., "Semi-Vektoren und Spinoren", Sitzungsber. Preuss. Akad. Wiss., 1932(XXXII), 522- 550, (1932). Related online version (cited on 30 October 2009): http://einstein-annalen.mpiwg-berlin.mpg.de/related_texts/sitzungsberichte/ VA2YYBC8. 7.3

[111] Einstein, A., and Mayer, W., "Die Diracgleichungen für Semivektoren", Proc. K. Akad. Wetensch., 36, 497-516, (1933). 7.3

[112] Einstein, A., and Mayer, W., "Spaltung der natürlichen Feldgleichungen für Semi-Vektoren in Spinor-Gleichungen vom Diracschen Typus", Proc. K. Akad. Wetensch., 36(2), 615-619, (1933). 7.3

[113] Einstein, A., and Mayer, W., "Darstellung der Semi-Vektoren als gewöhnliche Vektoren von besonderem Differentiationscharakter", Ann. Math., 35, 104-110, (1934). [DOI]. 7.3

[114] Eisenhart, L.P., "Fields of parallel vectors in the geometry of paths", Proc. Natl. Acad. Sci. $U S A, 8,207-212,(1922)$. [DOI]. 2

[115] Eisenhart, L.P., "Spaces with corresponding paths", Proc. Natl. Acad. Sci. USA, 8, 233-238, (1922). [DOI]. 2

[116] Eisenhart, L.P., "Another interpretation of the fundamental gauge vector of Weyl's theory of relativity", Proc. Natl. Acad. Sci. USA, 9, 175-178, (1923). [DOI]. 4.1.5

[117] Eisenhart, L.P., "The geometry of paths and general relativity", Ann. Math., 24, 367-392, (1923). [DOI]. 5

[118] Eisenhart, L.P., "Linear connections of a space which are determined by simply transitive continuous groups", Proc. Natl. Acad. Sci. USA, 11, 246-249, (1925). [DOI]. 6.4.1, 6.4.3

[119] Eisenhart, L.P., Riemannian Geometry, (Princeton University Press, Princeton, NJ, 1925). $34,6.4,7.2 .2$

[120] Eisenhart, L.P., "Einstein's recent theory of gravitation and electricity", Proc. Natl. Acad. Sci. USA, 12, 125-129, (1926). [DOI]. 6.1

[121] Eisenhart, L.P., Non-Riemannian Geometry, AMS Colloquium Publications, vol. VIII, (American Mathematical Society, Providence, RI, 1927). 2.1.2, 5, 6.4

[122] Eisenhart, L.P., and Veblen, O., "The Riemann Geometry and its generalizations", Proc. Natl. Acad. Sci. USA, 8, 19-23, (1922). [DOI]. 2, 5

[123] Eyraud, H., "La Théorie affine asymétrique du champs électromagnétique et gravifique et le rayonnement atomique", C. $R$. Acad. Sci., 180, 1245-1248, (1925). 5

[124] Eyraud, H., "Sur le charactère riemannien projectif du champ gravifique électromagnétique", C. R. Acad. Sci., 180, 127-129, (1925). 5

[125] Fabre, L., Les Théories d'Einstein, (Payot, Paris, 1921). Nouvelle éd. 10 
[126] Finsler, P., Über Kurven und Flächen in allgemeinen Räumen, Lehrbücher und Monographien aus dem Gebiete der exakten Wissenschaften: Mathematische Reihe, vol. 11, (Birkhäuser, Basel, 1951). Unveränderter Nachdruck der Dissertation von 1918. 2.1.1, 3.1

[127] Fisher, J.W., "The Wave Equation in Five Dimensions", Proc. R. Soc. London, Ser. A, 123, 489-493, (1929). 7.1, 7.2.4

[128] Fisher, J.W., and Flint, H.T., "The Equations of the Quantum Theory", Proc. R. Soc. London, Ser. A, 126, 644-653, (1930). 7.1

[129] Flint, H.T., "The First and Second Order Equations of the Quantum Theory", Proc. R. Soc. London, Ser. A, 124, 143-150, (1929). 7.1

[130] Fock, V.A., "Über die invariante Form der Wellen- und der Bewegungsgleichung für einen geladenen Massenpunkt", Z. Phys., 39, 226-232, (1926). [DOI]. 6.3.1, 7.2.2, 270

[131] Fock, V.A., "Geometrisierung der Diracschen Theorie des Elektrons", Z. Phys., 57, 261-277, (1929). [DOI]. 7.2.1, 7.2.2, 7.2.2, 7.2.2, 7.2.3

[132] Fock, V.A., "L'équation d'onde de Dirac et la Géometrie de Riemann", J. Phys. Radium, 10, 392-405, (1929). [DOI]. 7.2.2, 7.2.2, 7.2.3

[133] Fock, V.A., "Sur les équations de Dirac dans la théorie de relativité générale", $C$. $R$. Acad. Sci., 189, 25-27, (1929). 7.2.1, 7.2.2, 7.2.3

[134] Fock, V.A., and Ivanenko, D.D., "Géometrie quantique linéaire et déplacement parallèle", C. R. Acad. Sci., 188, 1470-1472, (1929). 7.2.2, 7.2.3

[135] Fock, V.A., and Ivanenko, D.D., "Über eine mögliche geometrische Deutung der relativistischen Quantentheorie", Z. Phys., 54, 798-802, (1929). [DOI]. 6.4.5, 7.2.2, 274

[136] Fölsing, A., Albert Einstein: Eine Biographie, (Suhrkamp, Frankfurt am Main, 1993). 1.1

[137] Förster, R., Beiträge zur spezielleren Theorie der Riemannschen P-Funktion, (Teubner, Leipzig, 1908). PhD Thesis, University of Leipzig. 322

[138] Fréedericksz, V., and Isakson, A., "Einige Bemerkungen über die Feldgeometrie", Phys. Z., 30, 645, (1929). 5

[139] French, A.P., ed., Einstein: A Centenary Volume, (Heinemann, London, 1979). 4.3.2

[140] Frenkel, J., "Die Wellenmechanik des rotierenden Elektrons und die Grundgleichnungen des elektromagnetischen Feldes", Z. Phys., 52, 356-363, (1928). 7.1, 7.2.4

[141] Frenkel, J., "Zur Wellenmechanik des rotierenden Elektrons", Z. Phys., 47, 786-803, (1928). [DOI] 7.2 .4

[142] Friedmann, A., and Schouten, J.A., "Über die Geometrie der halb-symmetrischen Übertragungen", Math. Z., 21, 211-223, (1924). [DOI]. 5, 6.2

[143] Goenner, H., "Unified field theories from Eddington and Einstein up to now", in de Sabbata, V., and Karade, T. M., eds., Relativistic Astrophysics and Cosmology, Vol. 1, Proceedings of the Sir Arthur Eddington Centenary Symposium, Nagpur, India, (World Scientific, Singapore, 1984). 2.2

[144] Goenner, H., and Havas, P., "Spherically-Symmetric Space-Times with vanishing Curvature Scalar", J. Math. Phys., 21, 1159-1167, (1980). [DOI]. 66 
[145] Goenner, H., and Wünsch, D., What did Kaluza and Klein contribute to Kaluza-Kleintheory?, 235, (Max Planck Institute for the History of Science, Berlin, 2003). 101, 6.3.1

[146] Goldstein, C., and Ritter, J., The Varieties of Unity: Sounding Unified Theories 1920-1930, 149, (Max Planck Institute for the History of Science, Berlin, 2000). 1.1

[147] Gonseth, F., and Juvet, G., "Les équations de l'électromagnétisme et l'équation de M. Schrödinger", C. R. Acad. Sci., 185, 535-538, (1927). eratum p. 732. 7.2.4

[148] Gonseth, F., and Juvet, G., "Sur la métrique de l'espace à cinq dimensions de l'électromagnétisme at de la gravitation", C. R. Acad. Sci., 185, 412-413, (1927). 7.2 .4

[149] Gonseth, F., and Juvet, G., "Sur l'équation de M. Schrödinger", C. R. Acad. Sci., 185, 448-450, (1927). eratum p. 624. 7.2.4

[150] Gonseth, F., and Juvet, G., "Sur les équations de l'électromagnétisme", C. R. Acad. Sci., 185, 341-343, (1927). eratum p. 483. 7.2 .4

[151] Gordon, W., "Der Strom der Diracschen Elektronentheorie", Z. Phys., 50, 630-632, (1928). [DOI]. 7.2 .4

[152] Gorelik, G., "Hermann Weyl and Large Numbers in Relativistic Cosmology", in Balashov, Y., and Vizgin, V., eds., Einstein Studies in Russia, Einstein Studies, vol. 10, pp. 91-106, (Birkhäuser, Boston; Basel, 2002). [Google Books]. 4.1.2

[153] Grommer, J., "Eine kleine Bemerkung zur neuen Einsteinschen Feldtheorie", Phys. Z., 30, $645,(1929) \cdot 6.4 .5$

[154] Grüning, M., Ein Haus für Albert Einstein: Erinnerungen, Briefe, Dokumente, (Verlag der Nation, Berlin, 1990). 6.4.4

[155] Hattori, K., "Über eine formale Erweiterung der Relativitätstheorie und ihren Zusammenhang mit der Theorie der Elektrizität", Phys. Z., 29, 538 - 549, (1928). 6.2

[156] Havas, P., "Einstein, Relativity and Gravitation Research in Vienna before 1938", in Goenner, H., Renn, J., Ritter, J., and Sauer, T., eds., The Expanding Worlds of General Relativity, Einstein Studies, vol. 7, pp. 161-206, (Birkhäuser, Boston; Basel, 1999). [Google Books]. 81

[157] Hehl, F.W., von der Heyde, P., Kerlick, G.D., and Nester, J.M., "General relativity with spin and torsion: Foundation and prospects", Rev. Mod. Phys., 48, 393-416, (1976). 45

[158] Heisenberg, W., and Pauli, W., "Zur Quantendynamik der Wellenfelder", Z. Phys., 56, 1-61, (1929). [DOI]. 7.1

[159] Hermann, A., Einstein: Der Weltweise und sein Jahrhundert. Eine Biographie, (Piper, Munich, 1994). 1.1

[160] Hessenberg, G., "Vektorielle Begründung der Differentialgeometrie", Math. Ann., 78, 187217, (1917). 3.1, 128

[161] Hilbert, D., "Die Grundlagen der Physik (Erste Mitteilung.)", Nachr. Koenigl. Gesellsch. Wiss. Goettingen, Math.-Phys. Kl., 1915, 395-407, (1915). Related online version (cited on 30 October 2009):

http://echo.mpiwg-berlin.mpg.de/content/modernphysics/hilbert/hilbert_ grundlagen_1915. 4.3.2 
[162] Hilbert, D., "Letter from David Hilbert", in Schulman, R., Kox, A.J., Janssen, M., and Illy, J., eds., Einstein Collected Papers, Vol. 8A, Doc 140, p. 195, (Princeton University Press, Princeton, NJ, 1997). 3.1

[163] Hoffmann, B., "Projective Relativity and the Quantum Field", Phys. Rev., 37, 88-89, (1930). 6.3 .2

[164] Hosokawa, T., "Connections in the manifold admitting generalized transformations", Proc. Imp. Acad. Japan, 8, 348-351, (1932). [DOI]. 154

[165] Infeld, L., "Bemerkungen zu der Arbeit von Herrn K. Hattori", Phys. Z., 29, 810-811, (1928). 6.2

[166] Infeld, L., "Zur Feldtheorie von Elektrizität und Gravitation”, Phys. Z., 29, 145-147, (1928). [DOI]. 6.2

[167] Infeld, L., and van der Waerden, B.L., "Die Wellengleichung des Elektrons in der allgemeinen Relativitätstheorie", Sitzungsber. Preuss. Akad. Wiss., 1933(IX), 380-401, (1933). 7.2.1, 7.3

[168] Infeld, L., and van der Waerden, B.L., "Die Wellengleichung des Elektrons in der allgemeinen Relativitätstheorie", Sitzungsber. Preuss. Akad. Wiss., 1933(IX), 380-401, (1933). 7.2.2

[169] Ivanenkov, D.D., and Fock, V.A., "Vorträge und Diskussionen der Theoretisch-Physikalischen Konferenz in Charkow (19.-25. Mai 1929)", Phys. Z., 30, 645-655, (1929). 7.2.2

[170] Jauch, J.M., and Rohrlich, F., The Theory of Photons and Electrons: The relativistic quantum field theory of charged particles with spin one-half, Texts and Monographs in Physics, (Springer, New York, 1980), 2nd edition. 139

[171] Jehle, H., "Zur allgemeinen relativistischen Quantenmechanik", Z. Phys., 87, 370-374, (1933). 7

[172] Jehle, H., "Zur allgemein-relativistischen Quantenmechanik II. Kosmologische Quantenerscheinungen", Z. Phys., 94, 692-706, (1935). [DOI]. 7

[173] Jordan, P., "Über die Polarisation der Lichtquanten", Z. Phys., 44, 292-300, (1927). 7.2.4

[174] Jordan, P., "Über Wellen und Korpuskeln in der Quantenmechanik", Z. Phys., 45, 766-775, (1927). [DOI]. 1.2

[175] Jordan, P., "Zur Quantenmechanik der Gasentartung”, Z. Phys., 44, 473-480, (1927). 1.2

[176] Jordan, P., Schwerkraft und Weltall, (Vieweg, Braunschweig, 1955), 2nd edition. 1.1

[177] Jordan, P., Albert Einstein: Sein Lebenswerk und die Zukunft der Physik, Wirkung und Gestalt, vol. 6, (Huber, Frauenfeld; Stuttgart, 1969). 1.1

[178] Jordan, P., and Klein, O., "Zum Mehrkörperproblem der Quantentheorie", Z. Phys., 45, 751-765, (1927). [DOI]. 1.2

[179] Jordan, P., and Wigner, E., "Über das Paulische 'Aquivalenzverbot"', Z. Phys., 47, 631-651, (1928). [DOI]. 1.2

[180] Juvet, G., "Les formules de Frenet pour un espace de M. Weyl", C. R. Acad. Sci., 172, 1647-1650, (1921). 4.1.5, 9 
[181] Kaluza, T., "Zum Unitätsproblem in der Physik", Sitzungsber. Preuss. Akad. Wiss., 1921, 966-972, (1921). Zum Unitätsproblem in der Physik. 2.4, 4.2, 7.2.4

[182] Kawaguchi, A., "Die Differentialgeometrie in der verallgemeinerten Mannigfaltigkeit", Rend. Circ. Mat. Palermo, 56, 245-276, (1932). [DOI]. 154

[183] Kirsten, C., and Treder, H.-J., Albert Einstein in Berlin 1913-1933, Teil I: Darstellungen und Dokumente, (Akademie-Verlag, Berlin, 1979). 1.1, 6.4.2, 192, 205, 7.2.3

[184] Klein, O., "The Atomicity of Electricity as a Quantum Law", Nature, 118, 516, (1926). [DOI]. 6.3.1, 7.2.4

[185] Klein, O., "Quantentheorie und fünfdimensionale Relativitätstheorie", Z. Phys., 37, 895-906, (1926). [DOI]. 4.2, 6.3.1, 6.3.2, 7.2.4

[186] Klein, O., "Elektrodynamik und Wellenmechanik vom Standpunkt des Korrespondenzprinzips", Z. Phys., 41, 407-442, (1927). [DOI]. 6.3.1

[187] Klein, O., "Elektrodynamik und Wellenmechanik vom Standpunkt des Korrespondenzprinzips", Z. Phys., 41, 407-442, (1927). [DOI]. 7.2.4

[188] Klein, O., "Sur l'article de M. L. de Broglie: 'L'univers à cinq dimensions et la méchanique ondulatoire"', J. Phys. Radium, 8, 242-243, (1927). [DOI]. 6.3.1

[189] Klein, O., "Zur fünfdimensionalen Darstellung der Relativitätstheorie", Z. Phys., 46, 188208, (1928). [DOI]. 6.3.1, 7.2.4

[190] Kojevnikov, A., "Dirac's Quantum Electrodynamics", in Balashov, Y., and Vizgin, V., eds., Einstein Studies in Russia, Einstein Studies, vol. 10, pp. 229-259, (Birkhäuser, Boston; Basel, 2002). [Google Books]. 1.2

[191] König, M., Herleitung und Untersuchung der Feld- und Bewegungsgleichungen von an das Gravitationsfeld gekoppelten Tensorfeld-Theorien, Masters Thesis, (Universität Göttingen, Göttingen, 2000). 64

[192] König, R., "Beiträge zu einer allgemeinen linearen Mannigfaltigkeitslehre", Jahresber. Deutsch. Math.-Verein., 28, 213-228, (1919). 3

[193] König, R., "Über affine Geometrie XXIV. Ein Beitrag zu ihrer Grundlegung", Ber. Saechs. Gesellsch. Wiss., 71, 1-19, (1919). 3.1

[194] Kopff, A., in Kopf, A., ed., Müller-Pouillets Lehrbuch der Physik, Vol. 5/2: Physik des Kosmos, (Vieweg, Braunschweig, 1928). 10

[195] Kosambi, D., "Affin-geometrische Grundlagen der einheitlichen Feldtheorie", Sitzungsber. Preuss. Akad. Wiss., 1932, 342-345, (1932). 6.2

[196] Kretschmann, E., "Über den physikalischen Sinn der Relativitätspostulate; A. Einsteins neue und seine ursprüngliche Relativitätstheorie", Ann. Phys. (Leipzig), 53, 592-614, (1917). 2.1.3.2, 9

[197] Kunii, S., "On a unified field theory of gravitational and electromagnetic fields", Mem. Coll. Sci. Kyoto Univ., Ser. A, 14, 195-212, (1931). 154

[198] Lanczos, C., "Die Erhaltungssätze in der feldmässigen Darstellung der Diracschen Theorie", Z. Phys., 57, 484-493, (1929). [DOI]. 1.2, 7.1 
[199] Lanczos, C., "Die tensoranalytischen Beziehungen der Diracschen Gleichung", Z. Phys., 57, 447-473, (1929). [DOI]. 7.1

[200] Lanczos, C., "Zur kovarianten Formulierung der Diracschen Gleichung", Z. Phys., 57, 474483, (1929). [DOI]. 7.1

[201] Lanczos, C., "Die neue Feldtheorie Einsteins", Ergeb. Exakten Naturwiss., 10, 97-132, (1931). [DOI]. 6.4.4, 8

[202] Lanczos, C., "Elektromagnetismus als natürliche Eigenschaft der Riemannschen Geometrie", Z. Phys., 73, 147-168, (1931). [DOI]. 6.4.4, 8

[203] Laporte, O., and Uhlenbeck, G.E., "Application of Spinor Analysis to the Maxwell and Dirac Equations", Phys. Rev., 37, 1380-1397, (1931). [DOI]. 7.3

[204] Levi-Civita, T., "Nozione di parallelismo in una varietà qualunque e conseguente specificazione geometrica della curvatura Riemanniana", Rend. Circ. Mat. Palermo, 42, 173-205, (1917). 2.1.3.2, 3.1

[205] Levi-Civita, T., Lezioni di calcolo differenziale assoluto, (Stock, Roma, 1925). 6.4.5, 10

[206] Levi-Civita, T., "A proposed modification of Einstein's field theory", Nature, 123, 678-679, (1929). [DOI]. 6.4.5

[207] Levi-Civita, T., "Vereinfachte Herstellung der Einsteinschen einheitlichen Feldgleichungen", Sitzungsber. Preuss. Akad. Wiss., 1929(IX), 137-153, (1929). 6.4.5, 7.2.2

[208] Levi-Civita, T., "Diracsche und Schrödingersche Gleichungen", Sitzungsber. Preuss. Akad. Wiss., 1933(V), 240-250, (1933). 7.2.2

[209] Lichnerowicz, A., Théories de la Gravitation et de L'Électromagnétisme, (Masson, Paris, 1955). 1.1

[210] Lopes Gagean, D., and Costa Leite, M., "A theoria de Kaluza-Klein", Analise (Lisboa), 5, 151-198, (1986). 6.3.1

[211] Lorentz, H.A., "The determination of the potentials in the general theory of relativity with some remarks about the measurement of lengths and intervals of time and about the theories of Weyl and Eddington", in Collected Papers, vol. 5, pp. 363-382, (Nijhoff, The Hague, 1937). Original paper in Proc. Acad. Amsterdam, 29 (1923) 383. 4.1.2, 4.3.1

[212] Ludwig, G., Fortschritte der projektiven Relativitätstheorie, (Vieweg, Braunschweig, 1951). 1.1

[213] Madelung, E., "Eine Übertragung der Diracschen Theorie des Elektrons in gewohnte Formen", Z. Phys., 54, 303-306, (1929). [DOI]. 7

[214] Maeterlick, M., Geheimnisse des Weltalls, (Deutsche Verlagsanstalt, Stuttgart, 1930). 6.4.2

[215] Majer, U., and Sauer, T., "Hilbert's 'World Equations' and His Vision of a Unified Science", in Kox, A.J., and Eisenstaedt, J., eds., The Universe of General Relativity, Einstein Studies, vol. 11, pp. 259-266, (Birkhäuser, Boston; Basel, 2005). [physics/0405110], [Google Books]. 4.3 .2

[216] Mandel, H., "Zur Herleitung der Feldgleichungen in der allgemeinen Relativitätstheorie. (Erste Mitteilung.)", Z. Phys., 39, 136-145, (1926). [DOI]. 6.3.1, 6.3.2 
[217] Mandel, H., "Zur Herleitung der Feldgleichungen in der allgemeinen Relativitätstheorie. (Zweite Mitteilung.)", Z. Phys., 45, 285-306, (1927). [DOI]. 6.3.2

[218] Mandel, H., "Über den Zusammenhang zwischen der Einsteinschen Theorie des Fernparallelismus und der fünfdimensionalen Feldtheorie", Z. Phys., 56, 838-844, (1929). [DOI]. 6.3.2, 6.4 .5

[219] Mandel, H., "Über den Zusammenhang zwischen der Einsteinschen Theorie des Fernparallelismus und der fünfdimensionalen Feldtheorie", Z. Phys., 56, 838-844, (1929). [DOI]. 6.3 .2

[220] Mandel, H., "Zur Axiomatik der fünfdimensionalen Relativitätstheorie", Z. Phys., 54, 564566, (1929). [DOI]. 6.3.1

[221] Mandel, H., "Zur tensoriellen Form der wellenmechanischen Gleichung des Elektrons", $Z$. Phys., 54, 567-570, (1929). [DOI]. 7.2.4

[222] Mandel, H., "Einige vergleichende Bemerkungen zur Quantentheorie des Elektrons", Z. Phys., 60, 782-794, (1930). [DOI]. 7.2.4

[223] Mastrobisi, G.J., "Il 'Manoscritto di Singapore' (1923) di Albert Einstein. Per una Teoria del 'Campo Unificato' tra possibilità fisica e necessità matematica”, Nuncius, 17, 269-305, (2002). 109

[224] Matsumoto, M., Foundations of Finsler Geometry and Special Finsler Spaces, (Kaiseisha Press, Ohtsu-shi, Japan, 1986). 2.1.1

[225] McVittie, G.C., "On Einstein's Unified Field Theory", Proc. R. Soc. London, Ser. A, 124, 366-374, (1929). 6.4.5

[226] McVittie, G.C., "Solution with axial symmetry of Einstein's equations of teleparallelism", Proc. Edinburgh Math. Soc., 2, 140, (1931). [DOI]. 6.4.5

[227] Michal, A.D., "Notes on scalar extensions of tensors and properties of local coordinates", Proc. Natl. Acad. Sci. USA, 17, 132-136, (1931). [DOI]. 6.3 .2

[228] Michal, A.D., and Botsford, J.L., "Extension of the New Einstein Geometry", Proc. Natl. Acad. Sci. USA, 18, 554-558, (1932). [DOI]. 6.3 .2

[229] Mie, G., "Grundlagen einer Theorie der Materie", Ann. Phys. (Leipzig), 37, 511-534, (1912). 3.1

[230] Mie, G., "Grundlagen einer Theorie der Materie. II", Ann. Phys. (Leipzig), 39, 1-40, (1912). 3.1

[231] Mie, G., "Grundlagen einer Theorie der Materie. III", Ann. Phys. (Leipzig), 40, 1-66, (1913). [DOI]. 3.1

[232] Mie, G., "Untersuchungen zum Problem der Quantenelektrik", Ann. Phys. (Leipzig), 85, 711-729, (1928). [DOI]. 7.1

[233] Milne, E.A. et al., "Physical Science and Philosophy", Nature, 139, 997-1008, (1937). 1.2

[234] Misner, C.W., Thorne, K.S., and Wheeler, J.A., Gravitation, (W.H. Freeman, San Francisco, 1973). 2.1.2

[235] Neugebauer, G., and Kramer, D., "Soliton concept in general relativity", Gen. Relativ. Gravit., 13, 195-200, (1981). [DOI]. 1.2 
[236] Neumann, E.R., Vorlesungen zur Einführung in die Relativitätstheorie, (Gustav Fischer, Jena, 1922). 10

[237] Newman, M.A.H., "A gauge-invariant tensor calculus", Proc. R. Soc. London, Ser. A, 116, 603-623, (1927). 4.1.5

[238] Nordström, G., "Über die Möglichkeit, das elektromagnetische Feld und das Gravitationsfeld zu vereinigen", Phys. Z., 15, 504-506, (1914). 2.4, 4.2

[239] Novobatzky, K., "Erweiterung der Feldgleichungen", Z. Phys., 72, 683-696, (1931). [DOI]. 11

[240] O'Raifeartaigh, L., and Straumann, N., "Gauge theory: Historical origins and some modern developments", Rev. Mod. Phys., 72, 1-23, (2000). [DOI]. 1.1, 323

[241] Pais, A., 'Subtle is the Lord': The Science and Life of Albert Einstein, (Oxford University Press, Oxford, 1982). 1.1, 102, 4.3.2, 160, 6.3.2, 6.4.1, 205, 6.4.4

[242] Palatini, A., "Intorno alla nuova teoria di Einstein", Rend. Lincei, 9, 633-639, (1929). 6.4.5

[243] Pauli, W., "Mercurperihelbewegung und Strahlenablenkung in Weyls Gravitationstheorie", Verh. Deutsch. Phys. Gesellsch., 21, 742-750, (1919). 4.1.5, 248

[244] Pauli, W., "Zur Theorie der Gravitation und Elektrizität von Hermann Weyl", Phys. Z., 20, 457-467, (1919). 4.1.4, 4.1.5

[245] Pauli, W., "Remark after Weyl's lecture in Bad Nauheim (86. Naturforscherversammlung, 19.-25. 9. 1920)", Phys. Z., 21, 650-651, (1920). 3.2

[246] Pauli, W., "Relativitätstheorie", in Sommerfeld, A., ed., Enzyklopädie der Mathematischen Wissenschaften mit Einschluss ihrer Anwendungen, Band V, 2. Teil, pp. 539-775, (Teubner, Leipzig, 1921). Related online version (cited on 30 October 2009):

http://gdz.sub. uni-goettingen.de/dms/load/img/?PPN=PPN360709672\&DMDID= dmdlog265. 1.1, 4, 4.1.4, 6.1, 9

[247] Pauli, W., "Zur Quantenmechanik des magnetischen Elektrons", Z. Phys., 43, 601-623, (1927). [DOI]. 7.2.4

[248] Pauli, W., "[Besprechung von] Band 10 der Ergebnisse der exakten Naturwissenschaften", Ergeb. Exakten Naturwiss., 11, 186-187, (1931). 6.4.4

[249] Pauli, W., "Über die Formulierung der Naturgesetze mit fünf homogenen Koordinaten. Teil I. Klassische Theorie", Ann. Phys. (Leipzig), 18, 305-336, (1933). 6.3.2, 6.3.2, 7.2.4

[250] Pauli, W., "Über die Formulierung der Naturgesetze mit fünf homogenen Koordinaten. Teil II. Die Diracschen Gleichungen für die Materiewellen", Ann. Phys. (Leipzig), 18, 337-372, (1933). 7.2.4

[251] Pauli, W., Wissenschaftlicher Briefwechsel mit Bohr, Einstein, Heisenberg u.a, Bd. I: 19191929, Sources in the History of Mathematics and Physical Sciences, vol. 2, (Springer, New York; Berlin, 1979). eds. Hermann, A. and von Meyenn, K. and Weisskopf, V.F. 4.3.3, 6.4.4

[252] Pauli, W., Wolfgang Pauli. Wissenschaftlicher Briefwechsel mit Bohr, Einstein, Heisenberg u.a, Bd. II: 1930-1939, (Springer, Berlin; New York, 1985). eds. Hermann, A. and von Meyenn, K. and Weisskopf, V.F. 7.2.2, 7.3 
[253] Pauli, W., and Solomon, J., "La théorie unitaire d'Einstein et Mayer et les équations de Dirac", J. Phys. Radium, 3, 452-663, (1932). 6.3.2, 7.2.4

[254] Pauli, W., and Solomon, J., "La théorie unitaire d'Einstein et Mayer et les équations de Dirac. II", J. Phys. Radium, 3, 582-589, (1932). 6.3.2

[255] Pauli, W., and Weisskopf, V., "Über die Quantisierung der skalaren relativistischen Wellengleichung", Helv. Phys. Acta, 7, 709-731, (1934). 7.3

[256] Penrose, R., and Rindler, W., Spinors and Space-Time, 2 vols., (Cambridge University Press, Cambridge, 1984). 56

[257] Penrose, R., and Rindler, W., Spinors and space-time, Vol. 1: Two-spinor calculus and relativistic fields, Cambridge Monographs on Mathematical Physics, (Cambridge University Press, Cambridge; New York, 1984). [Google Books]. 7.2.2

[258] Piaggio, H.T.M., "Einstein's and other Unitary Field Theories: An Explanation for the General Reader", Nature, 123(3109), 839-841, (1929). and ibid. 3110, 877-879. 6.4.2

[259] Podolsky, B., "A Tensor Form of Dirac's Equation", Phys. Rev., 37, 1398-1405, (1931). [DOI] . 7.2.1, 7.2.2

[260] Proca, A., "La nouvelle théorie d'Einstein", Bull. Math. Soc. Roumaine Sci., 1, 170-176, (1929). 6.4.3

[261] Proca, A., "La nouvelle théorie d'Einstein. II", Bull. Math. Soc. Roumaine Sci., 2, 15-22, (1929). 6.4.3

[262] Pyenson, L., "Physical Sense in Relativity: Max Planck Edits the Annalen der Physik, 19061918", in The Young Einstein: The Advent of Relativity, (Adam Hilger, Bristol; Boston, 1985). 3.1

[263] Rainich, G.Y., "Electrodynamics in General Relativity Theory", Proc. Natl. Acad. Sci. USA, 10, 124-127, (1924). 6.1

[264] Rainich, G.Y., "Second Note. Electrodynamics in General Relativity Theory", Proc. Natl. Acad. Sci. USA, 10, 294-298, (1924). 6.1

[265] Rainich, G.Y., "Electricity in Curved Space", Nature, 115, 498, (1925). [DOI]. 6.1, 8

[266] Rainich, G.Y., "Electrodynamics in general relativity", Trans. Amer. Math. Soc., 27, 106, (1925). [DOI]. 6.1

[267] Reichenbach, H., Philosophie der Raum-Zeit-Lehre, (de Gruyter, Berlin, 1928). 6.4.4, 10

[268] Reichenbach, H., "Zur Einordnung des neuen Einsteinschen Ansatzes über Gravitation und Elektrizität", Z. Phys., 53, 683-689, (1929). [DOI]. 6.4.4

[269] Reichenbächer, E., "3. Nachtrag zu der Arbeit Grundzüge zu einer Theorie der Elektrizität und der Gravitation", Ann. Phys. (Leipzig), 52, 174-178, (1917). 3.1, 3.1

[270] Reichenbächer, E., "Grundzüge zu einer Theorie der Elektrizität und der Gravitation", Ann. Phys. (Leipzig), 52, 134-173, (1917). 3.1, 3.1

[271] Reichenbächer, E., "Inwiefern lässt sich die moderne Gravitationstheorie ohne die Relativität begründen?", Die Naturwissenschaften, 8, 1008-1010, (1920). [DOI]. 3.1 
[272] Reichenbächer, E., "Schwere und Trägheit", Phys. Z., 22, 234-243, (1921). 4.1 .5

[273] Reichenbächer, E., "Eine neue Erklärung des Elektromagnetismus", Z. Phys., 13, 221-240, (1923). [DOI]. 2.1.1

[274] Reichenbächer, E., "Der Elektromagnetismus in der Weltgeometrie", Phys. Z., 27, 741-745, (1926). 3.1

[275] Reichenbächer, E., "Die Kopplung des Elektromagnetismus mit der Gravitation", Z. Phys., 44, 517-534, (1927). [DOI]. 6.3.1

[276] Reichenbächer, E., "Die fünfdimensionale Relativitätstheorie und das komplexe Linienelement", Z. Phys., 50, 425-432, (1928). [DOI]. 6.3.1, 7.2.4

[277] Reichenbächer, E., "Eine wellenmechanische Zweikomponententheorie", Z. Phys., 58, 402424, (1929). [DOI]. 7.2.2

[278] Reichenbächer, E., "Eine wellenmechanische Zweikomponententheorie. II", Z. Phys., 61, 490510, (1930). [DOI]. 7.2.2

[279] Renn, J., and Sauer, T., "Heuristics and Mathematical Representation in Einstein's Search for a Gravitational Field Equations", in Goenner, H., Renn, J., Ritter, J., and Sauer, T., eds., The Expanding Worlds of General Relativity, Einstein Studies, vol. 7, pp. 87-125, (Birkhäuser, Boston; Basel, 1999). [Google Books]. 11

[280] Renn, J. et al., ed., The Genesis of General Relativity, 4 vols., Boston Studies in the Philosophy of Science, vol. 250, (Springer, Dordrecht, 2007). 11

[281] Riemann, B., Über die Hypothesen, die der Geometrie zu Grunde liegen, (Wissenschaftliche Buchgesellschaft, Darmstadt, 1959). Reprint. 2.1.1

[282] Robertson, H.P., "Groups of motions in space admitting absolute parallelism", Ann. Math. (2), 33, 496-520, (1932). [DOI]. 6.3 .2

[283] Rosen, N., and Vallarta, M.S., "The spherically symmetrical field in the unified theory", Phys. Rev., 36, 110-120, (1930). [DOI]. 6.4.5

[284] Rosenfeld, L., "Zur Quantelung der Wellenfelder", Ann. Phys. (Leipzig), 5, 113-152, (1930). [DOI]. 1.2

[285] Rumer, G., "Form und Substanz", Z. Phys., 58, 273-279, (1929). [DOI]. 7.2.2, 8

[286] Rumer, G., "Zur Wellentheorie des Lichtquants", Z. Phys., 65, 244-252, (1930). [DOI]. 7.1

[287] Salkover, M., "The Unified Field Equations and Schwarzschild's Solution. II", Phys. Rev., 35, 214, (1930). [DOI]. 6.4.5

[288] Salkover, M., "The Unified Field-Theory and Schwarzschild's Solution", Phys. Rev., 35, 209, (1930). [DOI]. 6.4.5

[289] Sauer, T., "Field equations in teleparallel space-time: Einstein's Fernparallelismus approach toward unified field theory", Historia Math., 33, 399-439, (2006). [DOI], [physics/0405142]. 205, 207, 222

[290] Schmutzer, E., Relativistische Physik (Klassische Theorie), (Akademische Verlagsgesellschaft, Leipzig, 1968). 1.1 
[291] Scholz, E., "Spinor and gauge connections", Paper presented at the Sixth International Conference on the History of General Relativity, held in Amsterdam on June 26-29, 2002, conference paper. 7.2 .1

[292] Scholz, E., ed., Hermann Weyl's Raum-Zeit-Materie and a General Introduction to His Scientific Work, Proceedings of the DMV workshop, Oberwolfach 1993, DMV Seminar, vol. 30, (Birkhäuser, Boston; Basel, 2000). 1.1, 4

[293] Scholz, E., "Weyls Infinitesimalgeometrie, 1917-1925", in Scholz, E., ed., Hermann Weyl's Raum-Zeit-Materie and a General Introduction to His Scientific Work, Proceedings of the DMV workshop, Oberwolfach 1993, DMV Seminar, vol. 30, pp. 48-104, (Birkhäuser, Basel; Boston, 2000). 3.2

[294] Schouten, J.A., "Die direkte Analysis zur neueren Relativitätstheorie", Verh. K. Akad. Wetensch., 12(6), (1918). Printed as Separatum by Johannes Müller, Amsterdam. 3.1, 5

[295] Schouten, J.A., Die direkte Analysis zur neueren Relativitätstheorie, (Johannes Müller, Amsterdam, 1919). 4.1.4

[296] Schouten, J.A., "Nachtrag zur Arbeit 'Über die verschiedenen Arten der Übertragung in einer n-dimensionalen Mannigfaltigkeit, die einer Differentialgeometrie zugrundegelegt werden kann"', Math. Z., 15, 168, (1922). 5, 130, 9

[297] Schouten, J.A., "Über die verschiedenen Arten der Übertragung in einer n-dimensionalen Mannigfaltigkeit, die einer Differentialgeometrie zugrundegelegt werden kann", Math. Z., 13, 56-81, (1922). [DOI]. 5, 5, 9

[298] Schouten, J.A., "On a non-symmetrical affine field theory", Proc. K. Akad. Wetensch., 26, 850-857, (1923). 5, 5, 6.2, 6.2, 6.2

[299] Schouten, J.A., "Über die Einordnung der Affingeometrie in die Theorie der höheren Übertragungen. I", Math. Z., 17, 161-182, (1923). 9

[300] Schouten, J.A., Der Ricci-Kalkül, (Springer, Berlin, 1924). 2.1.2, 2.1.3.1, 2.1.3.2, 2.1.3.2, 2.1.3.2, $1,5,132,6.4 .3,10$

[301] Schouten, J.A., Raum, Zeit und Relativitätsprinzip, (Teubner, Leipzig und Berlin, 1924). 1.2

[302] Schouten, J.A., "Sur les connexions conformes et projectives de M. Cartan et la connexion linéaire générale de M. König", C. R. Acad. Sci., 178, 2044-2046, (1924). 3

[303] Schouten, J.A., "On a non-symmetrical affine field theory", Proc. K. Akad. Wetensch., 32, 105-108, (1929). 7.2.4

[304] Schouten, J.A., "Sur la signification géométrique de la propriété semi-symmétrique d'une connexion intégrale, qui laisse invariant le tenseur fondamental", C. R. Acad. Sci., 188, 1135-1136, (1929). 6.4.5

[305] Schouten, J.A., "Die Darstellung der Lorentzgruppe in der komplexen $E_{2}$ abgeleitet aus den Diracschen Zahlen", Proc. K. Akad. Wetensch., 38, 189-197, (1930). 7.2.2

[306] Schouten, J.A., "Dirac equation in general relativity. 1. Four dimensional theory", J. Math. Phys. (MIT), 10, 239-283, (1931). 7.2.1, 7.2.1, 7.2.2, 7.2.2, 7.2.4, 9

[307] Schouten, J.A., "Dirac Equations in General Relativity. 2. Five Dimensional Theory", J. Math. Phys. (MIT), 10, 272-283, (1931). 7.2.2, 7.2.4 
[308] Schouten, J.A., "Zur generellen Feldtheorie. Raumzeit und Spinraum. (G.F. V)", Z. Phys., 81, 405-417, (1933). [DOI]. 7.2.4, 7.3, 306, 9

[309] Schouten, J.A., "Zur generellen Feldtheorie. Semivektoren und Spinraum. (G.F. VII)", Z. Phys., 84, 92-111, (1933). [DOI]. 7.2.4, 7.3

[310] Schouten, J.A., Ricci-Calculus, (Springer, Berlin, 1954), 2nd edition. 2.1.2, 2.1.3.1, 2.1.3.2, 4.1.1

[311] Schouten, J.A., and Haantjes, J., "Generelle Feldtheorie VIII. Autogeodätische Linien und Weltlinien", Z. Phys., 89, 357-369, (1934). [DOI]. 17

[312] Schouten, J.A., and Hlavaty, V., "Zur Theorie der allgemeinen linearen Übertragung", Math. Z., 30, 414-432, (1929). [DOI]. 9

[313] Schouten, J.A., and van Dantzig, D., "Über unitäre Geometrie", Math. Ann., 103, 319-346, (1930). [DOI]. 2.3, 6.4.5

[314] Schouten, J.A., and van Dantzig, D., "Über eine vierdimensionale Deutung der neuesten Feldtheorie", Proc. K. Akad. Wetensch., 34, 1398-1407, (1931). 6.3.2

[315] Schouten, J.A., and van Dantzig, D., "Generelle Feldtheorie. (GF III)", Z. Phys., 78, 639667, (1932). [DOI]. 6.3.2, 178, 7

[316] Schouten, J.A., and van Dantzig, D., "Zum Unifizierungsproblem der Physik; Skizze einer generellen Feldtheorie. (GF I)", Proc. K. Akad. Wetensch., 35, 642-655, (1932). 6.3.2

[317] Schouten, J.A., and van Dantzig, D., "Zur generellen Feldtheorie; Diracsche Gleichung und Hamiltonsche Funktion. (GF II)", Proc. K. Akad. Wetensch., 35, 843-852, (1932). 6.3.2, 7.2 .4

[318] Schouten, J.A., and van Dantzig, D., "On projective connections and their application to the general field-theory. (GF. VI.)", Ann. Math., 34, 271-312, (1933). [DOI]. 6.3 .2

[319] Schrödinger, E., "Diracsches Elektron im Schwerefeld", Sitzungsber. Preuss. Akad. Wiss., 1932(XI), 105-128, (1932). 7.2.1, 7.2.2, 7.2.2, 7.2.4

[320] Schrödinger, E., "The final affine law II", Proc. R. Irish Acad. A, 51, 205-216, (1948). 2.1.1

[321] Schulman, R., Kox, A.J., Janssen, M., and Illy, J., eds., Einstein Collected Papers, Vol. 8A8B: The Berlin Years: Correspondence, 1914-1918, (Princeton University Press, Princeton, NJ, 1997). 3.1, 4.1.2, 4.1.3

[322] Schweber, S.S., QED and the men who made it: Dyson, Feynman, Schwinger, and Tomonaga, (Princeton University Press, Princeton, NJ, 1994). [Google Books]. 1.2

[323] Sen, N.R., "Der Energieinhalt des elektrischen Teilchens nach den Einsteinschen modifizierten Feldgleichungen", Z. Phys., 40, 667-674, (1927). [DOI]. 6.1

[324] Sen, N.R., "Das Keplerproblem der fünfdimensionalen Wellenmechanik und der Einfluss der Gravitation auf die Balmerformel", Z. Phys., 66, 689-692, (1930). [DOI]. 6.3.1

[325] Sen, N.R., "Die relativistisch-wellenmechanische Bewegung des materiellen Teilchens im homogenen Magnetfeld", Z. Phys., 66, 693-696, (1930). [DOI]. 6.3.1

[326] Sigurdsson, S., Hermann Weyl, Mathematics and Physics, 1900-1927, Ph.D. Thesis, (Harvard University, Cambridge, 1991). 7.2.2, 9 
[327] Sommerfeld, A., "Letter to H. Weyl", in Schulman, R., Kox, A.J., Janssen, M., and Illy, J., eds., Einstein Collected Papers, Vol. 8B, Doc 619 Note 18, (Princeton University Press, Princeton, NJ, 1997). 4.1.4

[328] Stachel, J., "The Rise and Fall of Geometrodynamics", in Schaffner, K.F., and Cohen, R.S., eds., PSA 1972, Proceedings of the 1972 Biennial Meeting of the Philosophy of Science Association, Boston Studies in the Philosophy of Science, vol. XX, pp. 31-54, (Reidel, Dordrecht; Boston, 1974). [Google Books]. 326

[329] Stachel, J., "The other Einstein: Einstein contra Field Theory", Sci. Context, 6, 275-290, (1993). [DOI]. 70

[330] Stachel, J., Einstein from 'B' to ' $Z$ ', Einstein Studies, vol. 9, (Birkhäuser, Boston; Basel, 2002). [Google Books]. 1.1, 4.3.2, 152, 6.4.4

[331] Straneo, P., "Gleichungen zu einer einheitlichen Feldtheorie", Sitzungsber. Preuss. Akad. Wiss., 1931(XIII), 319-325, (1931). 6.2

[332] Straneo, P., "Teorie unitarie della gravitazione e dell'electricita”, Nuovo Cimento, 8, 125-145, (1931). 6.2

[333] Straneo, P., "I tensori energetici nella teoria unitaria a geometrizzazione assoluta", Rend. Lincei, 15, 563-568, (1932). 6.2

[334] Straumann, N., "Zum Ursprung der Eichtheorien bei Hermann Weyl", Phys. Blaetter, 43, 414-421, (1987). 1.1

[335] Straumann, N., "Ursprünge der Eichtheorien", in Scholz, E., ed., Hermann Weyl's RaumZeit-Materie and a General Introduction to His Scientific Work, Proceedings of the DMV workshop, Oberwolfach 1993, DMV Seminar, vol. 30, (Birkhäuser, Basel; Boston, 2001). 1.1, 323

[336] Struik, D.J., Grundzüge der mehrdimensionalen Geometrie in direkter Darstellung, (Springer, Berlin, 1922). 5

[337] Struik, D.J., Theory of Linear Connections, Ergebnisse der Mathematik und ihrer Grenzgebiete, vol. 3, (Springer, Berlin, 1934). 5

[338] Struik, D.J., and Wiener, N., "A relativistic theory of Quanta", J. Math. Phys. (MIT), 7, $1-23,(1927) .7$

[339] Swann, W.F.G., "Statement in regard to professor Einstein's publications", Science, 71, 390-391, (1930). [DOI]. 6.4.3

[340] Tamm, I., "Die Einsteinsche einheitliche Feldtheorie und die Quantentheorie", Phys. Z., 30, 652-654, (1929). 7.2.3

[341] Tamm, I., "Über den Zusammenhang der Einsteinschen einheitlichen Feldtheorie mit der Quantentheorie", Proc. R. Acad. Amsterdam, 32, 288-291, (1929). 7.2.3

[342] Tamm, I., and Leontowitsch, M., "Bemerkungen zur Einsteinschen einheitlichen Feldtheorie", Z. Phys., 57, 354-366, (1929). [DOI]. 6.4.5

[343] Temple, G., "The Tensorial Form of Dirac's Wave Equation", Proc. R. Soc. London, Ser. A, 122, 352-357, (1929). 7 
[344] Tetrode, H., "Allgemein-relativistische Quantentheorie des Elektrons", Z. Phys., 50, 336$346,(1928)$. [DOI]. 6.4.5, 7.2.2

[345] Tetrode, H., "Der Impuls-Energiesatz in der Diracschen Quantentheorie des Elektrons", $Z$. Phys., 49, 858-864, (1928). [DOI]. 239

[346] Thomas, J.M., "On various geometries giving a unified electric and gravitational theory", Proc. Natl. Acad. Sci. USA, 12, 187-191, (1926). [DOI]. 2.1.3.2, 6.1, 6.1, 6.2

[347] Thomas, T.Y., "Note on the projective geometry of paths", Bull. Am. Math. Soc., 27, 318$322,(1925)$. [DOI]. 2

[348] Thomas, T.Y., "On the projective and equi-projective geometry of paths", Proc. Natl. Acad. Sci. USA, 11, 199-203, (1925). 2.1.2, 2

[349] Thomas, T.Y., "On the projective and equi-projective geometry of paths", Proc. Natl. Acad. Sci. USA, 11, 199-203, (1925). 2

[350] Thomas, T.Y., "On the unified field theory. I", Proc. Natl. Acad. Sci. USA, 16, 761-776, (1930). 6.4.5

[351] Thomas, T.Y., "On the unified field theory. II", Proc. Natl. Acad. Sci. USA, 16, 830-835, (1930). 6.4.5

[352] Thomas, T.Y., "On the unified field theory. III", Proc. Natl. Acad. Sci. USA, 17, 48-58, (1931). 6.4 .5

[353] Thomas, T.Y., "On the unified field theory. IV", Proc. Natl. Acad. Sci. USA, 17, 111-119, (1931). 6.4.5

[354] Thomas, T.Y., "On the unified field theory. V", Proc. Natl. Acad. Sci. USA, 17, 199-210, (1931). 6.4.5

[355] Thomas, T.Y., "On the unified field theory. VI", Proc. Natl. Acad. Sci. USA, 17, 325-329, (1931). 6.4.5

[356] Tonnelat, M.A., La théorie du champ unifié d'Einstein et quelques-uns de ses développements, (Gauthier-Villars, Paris, 1955). 1.1, 1.2, 2.1.1, 32, 36, 2.1.3.1

[357] Treder, H.-J., "Der heutige Stand der Geometrisierung der Physik und der Physikalisierung der Geometrie", Sitzungsber. Akad. Wiss. DDR, Math.-Nat.-Technik, 1975(XIV), 1-33, (1975). 140

[358] Vallarta, M.S., "On Einstein's unified field equations and the Schwarzschild solution", Proc. Natl. Acad. Sci. USA, 15, 784-788, (1929). [DOI]. 6.4.5

[359] van Dantzig, D., "Die Wiederholung des Michelson Versuch's und die Relativitätstheorie", Math. Ann., 96, 261-228, (1926). 177

[360] van Dantzig, D., "Theorie des projektiven Zusammenhangs n-dimensionaler Räume", Math. Ann., 106, 400-454, (1932). 6.3.2

[361] van Dantzig, D., "Zur allgemeinen projektiven Differentialgeometrie. I. Einordnung der Affingeometrie", Proc. K. Akad. Wetensch., 35, 524-534, (1932). 6.3.2

[362] van Dantzig, D., "Zur allgemeinen projektiven Differentialgeometrie. II. $X_{n+1}$ mit eingliedriger Gruppe", Proc. K. Akad. Wetensch., 35, 525-542, (1932). 6.3.2 
[363] van Dantzig, D., "Electromagnetism, independent of metrical geometry. 1. The foundations", Proc. K. Akad. Wetensch., 37, 521-525, (1934). 1.2, 188, 8

[364] van Dantzig, D., "Electromagnetism, independent of metrical geometry. 2. Variational Principles and further generalization of the theory", Proc. K. Akad. Wetensch., 37, 526-531, (1934). 1.2, 188

[365] van Dantzig, D., "Electromagnetism, independent of metrical geometry. 3. Mass and motion", Proc. K. Akad. Wetensch., 37, 644-652, (1934). 1.2, 188

[366] van Dantzig, D., "Electromagnetism, independent of metrical geometry. 4. Momentum and energy: waves", Proc. K. Akad. Wetensch., 37, 825-863, (1934). 1.2, 188

[367] van Dantzig, D., "The fundamental equations of electromagnetism, independent of metrical geometry", Proc. Cambridge Philos. Soc., 30, 421-427, (1934). [DOI]. 1.2, 188

[368] van der Waerden, B.L., "Spinoranalysis", Nachr. Koenigl. Gesellsch. Wiss. Goettingen, Math.-Phys. Kl., 1929, 100-106, (1929). 6.3.2, 7.2.1, 7.3, 7.3

[369] van der Waerden, B.L., Die gruppentheoretische Methode in der Quantenmechanik, (Springer, Berlin, 1932). 7.2.1

[370] van der Waerden, B.L., A History of Algebra, (Springer, Berlin; New York, 1980). 308

[371] van Dongen, J., Einstein's Unification: General relativity and the Quest for Mathematical Naturalness, Ph.D. Thesis, (University of Amsterdam, Amsterdam, 2002). 1.1, 140, 7.3, 308

[372] van Dongen, J., "Einstein's Methodology, Semivectors and the Unification of Electrons and Protons", Arch. Hist. Exact Sci., 58, 219-254, (2003). 7.3

[373] Veblen, O., "Projective and affine geometry of paths", Proc. Natl. Acad. Sci. USA, 8, 347$350,(1922)$. [DOI]. 2

[374] Veblen, O., "Projective tensors and connections", Proc. Natl. Acad. Sci. USA, 14, 154-166, (1928). [DOI]. 6.3.2

[375] Veblen, O., "A generalization of the quadratic differential form", Quart. J. Math., 1, 60, (1929). 6.3 .2

[376] Veblen, O., "Projective tensors and connections", J. London Math. Soc., 4, 140-160, (1929). 6.3 .2

[377] Veblen, O., "Geometry of four-component Spinors", Proc. Natl. Acad. Sci. USA, 19, 503-517, (1933). 7.2 .1

[378] Veblen, O., "Geometry of two-component Spinors", Proc. Natl. Acad. Sci. USA, 19, 462-474, (1933). 7.2.1, 7.2 .1

[379] Veblen, O., Projektive Relativitätstheorie, Ergebnisse der Mathematik und ihrer Grenzgebiete, vol. 2, (Springer, Berlin, 1933). 49, 6.3 .2

[380] Veblen, O., "Spinors in Projective Relativity", Proc. Natl. Acad. Sci. USA, 19, 979-989, (1933). [DOI]. 7.2.1

[381] Veblen, O., and Hoffmann, B., "Projective relativity", Phys. Rev., 36, 810-822, (1930). [DOI]. 6.3 .2 
[382] Veblen, O., and Taub, A.H., "Projective differentiation of spinors", Proc. Natl. Acad. Sci. USA, 20, 85-92, (1933). 7.2.1

[383] Veblen, O., and Thomas, T.Y., "The geometry of paths", Trans. Amer. Math. Soc., 25, 551-608, (1923). [DOI]. 5

[384] Vizgin, V.P., "The geometrical Unified Field Program", in Howard, D., and Stachel, J., eds., Einstein and the History of General Relativity, Based on the proceedings of the 1986 Osgood Hill Conference, North Andover, Massachusetts, 8-11 May, Einstein Studies, vol. 1, pp. 300-314, (Birkhäuser, Boston; Basel, 1989). 1.1

[385] Vizgin, V.P., Unified Field Theories in the first third of the 20th century, (Birkhäuser, Basel; Boston, 1994). [Google Books]. 1.1, 4, 5, 7, 11

[386] von Laue, M., Die Relativitätstheorie. Band 2: Allgemeine Relativitätstheorie und Einsteinsche Gravitationstheorie, (Vieweg, Braunschweig, 1921). 9

[387] von Laue, M., Die Relativitätstheorie. Band 2: Die allgemeine Relativitätstheorie. Einstein's Lehre von der Schwerkraft, (Vieweg, Braunschweig, 1923), 2nd edition. 62, 10

[388] von Wisniewski, F.J., "Die Diracschen und Maxwellschen Differentialgleichungen", Z. Phys., 63, 713-717, (1930). 7.1

[389] Wald, R.M., General Relativity, (University of Chicago Press, Chicago, 1984). 2.1.2

[390] Watanabe, S., "Reversibility of Quantum-Electrodynamics", Phys. Rev., 84, 1008-1025, (1951). [DOI]. 139

[391] Weitzenböck, R., "Über die Wirkungsfunktion in der Weyl'schen Physik. I., II.", Sitzungsber. Akad. Wiss. Wien, 1920, 638-708, (1920). 4.1 .1

[392] Weitzenböck, R., "Neuere Arbeiten der algebraischen Invariantentheorie. Differentialinvarianten", in Meyer, W.R., and Mohrmann, H., eds., Enzyklopädie der Mathematischen Wissenschaften mit Einschluss ihrer Anwendungen, Band III, 3. Teil, D 10, pp. 1-71, (Teubner, Leipzig, 1921). Related online version (cited on 30 October 2009): http://gdz.sub. uni-goettingen . de/dms/load/img/?PPN=PPN360610161\&DMDID= dmdlog323. 9

[393] Weitzenböck, R., "Differentialinvarianten in der Einsteinschen Theorie des Fernparallelismus", Sitzungsber. Preuss. Akad. Wiss., 1928(XXVI), 466-474, (1928). 6.4.1

[394] Wenzl, A., Das naturwissenschaftliche Weltbild der Gegenwart, (Quelle und Meyer, Leipzig, 1929). 10

[395] Weyl, H., "Gravitation und Elektrizität", Sitzungsber. Preuss. Akad. Wiss., 1918(XXVI), 465-478, (1918). With a "Nachtrag" of Einstein, p. 478, and "Erwiderung des Verfassers", pp. $478-480.2 .1 .3 .2,4.1 .2$

[396] Weyl, H., Raum, Zeit, Materie, (Springer, Berlin, 1918). 4.1.1, 4.1.2, 4.1.3, 10

[397] Weyl, H., "Reine Infinitesimalgeometrie", Math. Z., 2, 384-411, (1918). [DOI]. 2.1.3.2, 2.1.3.2, $3.1,4.1 .1,4.1 .1,82,4.1 .2,128$

[398] Weyl, H., Raum, Zeit, Materie, (Springer, Berlin, 1919), 3rd edition. 3.1, 4.1.2, 4.1.5, 4.3.3, 128 
[399] Weyl, H., "Elektrizität und Gravitation", Phys. Z., 21, 649-650, (1920). 4.1.5, 4.1.5

[400] Weyl, H., "Feld und Materie", Ann. Phys. (Leipzig), 65, 541-563, (1921). [DOI]. 4.1.5

[401] Weyl, H., Raum, Zeit, Materie, (Springer, Berlin, 1921), 4th edition. 2.1.3.2, 4.1.5

[402] Weyl, H., "Über die physikalischen Grundlagen der erweiterten Relativitätstheorie", Phys. $Z$., 22, 473-480, (1921). 4.1.5, 4.1.5

[403] Weyl, H., "Zur Infinitesimalgeometrie: Einordnung der projektiven und konformen Auffassung", Nachr. Koenigl. Gesellsch. Wiss. Goettingen, Math.-Phys. Kl., 1921, 99-112, (1921). 2.1 .2

[404] Weyl, H., Mathematische Analyse des Raumproblems, (Springer, Berlin, 1923). 2.1.2

[405] Weyl, H., Raum, Zeit, Materie, (Springer, Berlin, 1923), 5th edition. 4.3.3

[406] Weyl, H., Gruppentheorie und Quantenmechanik, (Hirzel, Leipzig, 1928). 7.2.2, 7.2.2

[407] Weyl, H., "Elektron und Gravitation I", Z. Phys., 56, 330-352, (1929). [DOI]. 4.1.4, 6.4.4, $7.2 .1,7.2 .2,7.2 .2,7.2 .2$

[408] Weyl, H., "Gravitation and the electron", Proc. Natl. Acad. Sci. USA, 15, 323-334, (1929). [DOI]. 4.1.4, 7, 7.2.1, 7.2.2, 7.2.2, 7.2.2

[409] Weyl, H., The Theory of Groups and Quantum Mechanics, (Dover, New York, 1950). [Google Books]. Translated from the 2nd rev. German ed. 1931. 12

[410] Weyl, H., Space, Time, Matter, (Dover Publications, New York, 1952), 4th edition. [Google Books]. 4.1.4

[411] Weyl, H., Gesammelte Abhandlungen, (Springer, Berlin; New York, 1968). [Google Books]. 4 vols., K. Chandrasekharan, ed. 19

[412] Wheeler, J.A., Geometrodynamics, Topics of Modern Physics, vol. 1, (Academic Press, New York, 1962). 1.2

[413] Whitehead, J.H.C., "The representation of projective spaces", Ann. Math., 32, 327-360, (1931). [DOI]. 6.3.2

[414] Whittaker, E.T., A History of the Theories of Aether and Electricity, Vol. 2: The Modern Theories 1900-1926, (Nelson \& Sons, London, 1953). 1.1

[415] Whittaker, J.M., "On the principle of least action in wave mechanics", Proc. R. Soc. London, Ser. A, 121, 543-557, (1928). 7, 256, 7, 7.2.1

[416] Wiener, N., and Vallarta, M.S., "On the spherically symmetric statical field in Einstein's unified theory of electromagnetism and gravitation", Proc. Natl. Acad. Sci. USA, 15, 353$356,(1929) .6 .4 .5$

[417] Wiener, N., and Vallarta, M.S., "On the spherically symmetric statical field in Einstein's unified theory of electromagnetism and gravitation: A correction", Proc. Natl. Acad. Sci. USA, 15, 802-804, (1929). 6.4.5

[418] Wiener, N., and Vallarta, M.S., "Unified field theory with electricity and gravitation", Nature, 123, 317, (1929). [DOI]. 7.2.3 
[419] Wigner, E., "Eine Bemerkung zu Einsteins neuer Formulierung des allgemeinen Relativitätsprinzips", Z. Phys., 53, 592-596, (1929). [DOI]. 6.4.5, 7.2.2

[420] Wilson, W., "Relativity and Wave Mechanics", Proc. R. Soc. London, Ser. A, 118, 441-448, (1928). 7.2 .4

[421] Wirtinger, W., "On a general infinitesimal geometry in reference to the theory of relativity", Trans. Cambridge Philos. Soc., 22, 439-448, (1922). 99

[422] Witten, L., "A geometric theory of electromagnetic and gravitational fields", in Witten, L., ed., Gravitation: An Introduction to Current Research, (Wiley, New York, 1962). 8

[423] Wünsch, D., Theodor Kaluza (1885-1954). Leben und Werk, 2 vols., Ph.D. Thesis, (Universitäet Stuttgart, Stuttgart, 2000). 8

[424] Yang, C.N., "Hermann Weyl's Contribution to Physics", in Chandrasekharan, K., ed., Hermann Weyl, 1885-1985: Centenary lectures delivered by C.N. Yang, R. Penrose, A. Borel at the ETH Zürich, pp. 7-21, (Springer, Berlin; New York, 1986). [Google Books]. 7.2.2

[425] Yano, K., The theory of Lie derivatives and its applications, (North-Holland, Amsterdam, 1955). 27, 2.1.5.3

[426] Zaycoff, R., "Fernparallelismus und Wellenmechanik. I", Z. Phys., 58, 833-840, (1929). [DOI]. 7.2 .2

[427] Zaycoff, R., "Fernparallelismus und Wellenmechanik. II", Z. Phys., 59, 110-113, (1929). [DOI] 7.2.2

[428] Zaycoff, R., "Zu der neuesten Formulierung der Einsteinschen einheitlichen Feldtheorie", $Z$. Phys., 56, 717-726, (1929). [DOI]. 6.4.5, 7

[429] Zaycoff, R., "Zur Begründung einer neuen Feldtheorie von A. Einstein", Z. Phys., 53, 719728, (1929). [DOI]. 6.4.5

[430] Zaycoff, R., "Zur Begründung einer neuen Feldtheorie von A. Einstein. (Dritte Mitteilung.)", Z. Phys., 54, 738-740, (1929). [DOI]. 6.4.5

[431] Zaycoff, R., "Zur Begründung einer neuen Feldtheorie von A. Einstein. (Zweite Mitteilung.)", Z. Phys., 54, 590-593, (1929). [DOI]. 6.4.5

[432] Zaycoff, R., "Zur neuen Quantentheorie", Z. Phys., 54, 588-589, (1929). [DOI]. 7

[433] Zaycoff, R., "Das relativistische Elektron", Z. Phys., 61, 395-410, (1930). [DOI]. 6.4.3, 7.2.2

[434] Zaycoff, R., "Über eine allgemeine Form der Diracschen Gleichung", Ann. Phys. (Leipzig), 7, 650-660, (1930). [DOI]. 7.2.2, 7.2.4 